Florida International University FIU Digital Commons

\title{
Role of carbon nanotube dispersion in fracture toughening of plasma sprayed aluminum oxide - carbon nanotube nanocomposite coating
}

Kantesh Balani

Florida International University

DOI: $10.25148 /$ etd.FI14050417

Follow this and additional works at: https://digitalcommons.fiu.edu/etd

Part of the Mechanical Engineering Commons

\section{Recommended Citation}

Balani, Kantesh, "Role of carbon nanotube dispersion in fracture toughening of plasma sprayed aluminum oxide - carbon nanotube nanocomposite coating" (2007). FIU Electronic Theses and Dissertations. 1380.

https://digitalcommons.fiu.edu/etd/1380 
FLORIDA INTERNATIONAL UNIVERSITY

Miami, Florida

ROLE OF CARBON NANOTUBE DISPERSION IN FRACTURE TOUGHENING OF PLASMA SPRAYED ALUMINUM OXIDE - CARBON NANOTUBE NANOCOMPOSITE COATING

A dissertation submitted in partial fulfillment of the requirements for the degree of DOCTOR OF PHILOSOPHY

in

MECHANICAL ENGINEERING

by

Kantesh Balani 
To: Dean Vish Prasad

College of Engineering and Computing

This dissertation, written by Kantesh Balani, and entitled Role of Carbon Nanotube Dispersion in Fracture Toughening of Plasma Sprayed Aluminum Oxide - Carbon Nanotube Nanocomposite Coating, having been approved in respect to style and intellectual content, is referred to you for judgment.

We have read this dissertation and recommend that it be approved.

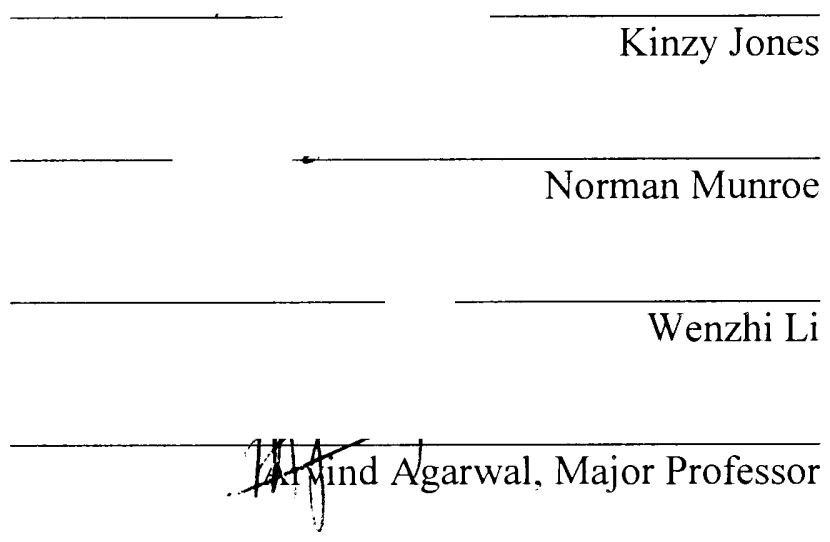

Date of Defense: May 10, 2007

The dissertation of Kantesh Balani is approved.

Dean Vish Prasad

College of Engineering and Computing

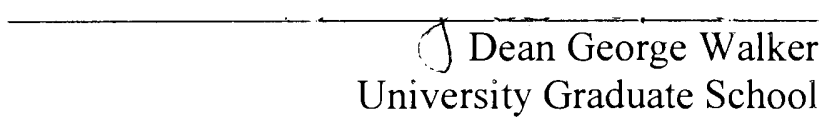

Florida International University, 2007 


\section{DEDICATION}

This dissertation is dedicated to everloving memory of my father. He would have been extremely proud of this moment. I dedicate this dissertation to my parents and my maternal uncle. 


\section{ACKNOWLEDGMENTS}

I sincerely thank Dr. Arvind Agarwal for his critical guidance through the ups and downs of this journey. I sincerely thank him for serving as chair for my dissertation committee. His untiring support and encouragement throughout last four years has made the path very clear and easy. I thank his time for long discussion-hours he dedicated just to clear the knots in this research. His ideas always gave a new dimension to the research, and proposed many different aisles of solution. I learned lot from his teaching. His dynamic delivery of lectures not only created interest, but also challenged me to explore further. He trained us gradually to master what we do, not only as a professional, but also as a person. He taught us the meaning of doing the right thing, performed in a right manner at the right time to achieve maximum output. His ever-enthusiastic spirit, critical perspicacity, and energetic approach were the sole motivation of my persistence. He is the person I look upto, a person who leads by example. He has taught me to challenge myself and stretch my limits. Words are just not enough to thank his deeds and concerns. Without him, this dissertation would not have even existed or thought of.

I extend my heartfelt thanks to Dr. Kinzy W. Jones not only for time in serving the dissertation committee, but also his continual mentoring throughout. Much learning was through his informative classes and his ability to cover obscure and abstract concepts in no time. His vast knowledge and experience, which he also shared during my proposal defense, were of great value. It encouraged me to dwell more in research and develop strategies of applying new ideas in my research. He encouraged my training on advanced characterization tools, which I applied extensively during my research. Amid his busy schedule he always had time to say a quick hello with a smile and heeded to what I had to 
say. His words were always encouraging and supporting and helped me nurture my confidence. Humbly, I thank him for all that he is to me.

I sincerely thank Dr. Wenzhi Li for serving in the dissertation committee. His smile and encouragement always uplifted my spirit and helped me maintain interest and tempo in my research. Research meetings and his constructive ideas helped me develop a new direction of my research. He always had time to encourage and support my ideas, endorse the research work and appreciate the results I got. His belief in my capabilities helped me develop self-reliance and independence in what I did. I sincerely thank him for his encouragement and support.

I thank Dr. Norman Munroe for his ever-smiling approach and encouragement throughout. He always had been very supportive and was always available if I had any questions. His tireless working taught me to work effectively under stress. He always had been one of the pillars who strengthened me during the time of need. He extended his research facilities whemever I had any problems finding a source. His help during such times made the path of research much smoother. I sincerely thank him for his time and concern in serving the dissertation committee.

I thank Dr. Lawrence T. Kabacoff for his time and support during the research project. This work was done under Office of Naval Research (ONR) grant number N00014-05-1-0398.

I sincerely thank Dr. S. Saxena, Director, CeSMEC (Center for the Study of Matter under Extreme Conditions), FIU, for extending Raman spectroscopy facilities. I appreciate his time and words of motivation during this research. I thank Dr. N. B. 
Dahotre for his discussion on the future work of this dissertation. His encouragement during this research also kept me motivated.

I thank Prof. Sudipta Seal, Director, Surface Engineering and Nanotechnology Laboratory, Mr. Viswanathan, Mr. Satya and Mr. Samir at University of Central Florida (UCF), for helping with TEM of samples.

I thank Dr. Tapas Laha for his support throughout. His presence always encouraged me to follow the standards he had created. Mimicking his ability to question logically and work tirelessly made me do things better in a very systematic way. I sincerely thank Mr. Srinivasa R. Bakshi for his informative discussions and spears of ideas. His presence made office environment much more calmer and enjoyable. I also thank Dr. Yao Chen, Ms. Gabriela Gonzalez, Ms. Melanie Andara, Mr. Jorge Tercero, Mr. Anup Keshri, Mr. Sunil Anand Musali, and Mr. Venkata Pasumarthi for their time and support. I also thank Mr. Harindra Vedala, Mr. Srinivas Kulkarni, Mr. Srikant, Dr. S. Roy, Mr. Revansidha Gulve, and Mr. Amit Datye for their support during my research.

I thank Dr. Yanquin Liu during his consistent assistance with Scanning Electron Microscopy and Transmission Electron Microscopy. More, I thank his tutelage and camaraderie throughout this period. His supportive words, constructive comments and interactive ideas helped me overcome a lot of hurdles during my research and personal life. I heartly thank him.

I thank the Mechanical and Materials Engineering Department for extending support. I thank the University Graduate School for extending a Dissertation Year Fellowship to support my research. 
I would like to thank Ms. Birgit Olkuch, Mr. Prasanna Maloji, Mr. Naveen Savaram, Mr. Tushar Sawant, Mr. Amol Kumar Kolhe, Mr. Srinivasarao Boddepalli, Ms. Rachna Mehrotra, Mr. Shadab Anwar, Ms. Monica Joshi, Mr. Alok Bhute, and Mr. Rameshwar Ajja for their tacit support, thoughtful concern, and unselfish care during hard times. I also thank Mr. Navneet Pate and Ms. Sonali Pate for their kind support. They all pulled me together whenever I was down. They were a source of strength in me. I extend my heartfelt thank to Mr. Dev Vachhani for all his time, words of encouragement and mental support. He was one of the pillars who kept me still and intact during my research. Thank is so small a word to express my gratitude for him.

Finally I would thank my mother who stood beside me and supported me full heartedly. Her unmatched sacrifice, unlimited love and affectionate blessing have made me successfully complete the degree. 
ABSTRACT OF THE DISSERTATION

ROLE OF CARBON NANOTUBE DISPERSION IN FRACTURE TOUGHENING OF

PLASMA SPRAYED ALUMINUM OXIDE - CARBON NANOTUBE

NANOCOMPOSITE COATING

by

Kantesh Balani

Florida International University, 2007

Miami, Florida

Professor Arvind Agarwal, Major Professor

Aluminum oxide $\left(\mathrm{Al}_{2} \mathrm{O}_{3}\right.$, or alumina) is a conventional ceramic known for applications such as wear resistant coatings, thermal liners, heaters, crucibles, dielectric systems, etc. However applications of $\mathrm{Al}_{2} \mathrm{O}_{3}$ are limited owing to its inherent brittleness. Due to its excellent mechanical properties and bending strength, carbon nanotubes (CNT) is an ideal reinforcement for $\mathrm{Al}_{2} \mathrm{O}_{3}$ matrix to improve its fracture toughness.

The role of CNT dispersion in the fracture toughening of the plasma sprayed $\mathrm{Al}_{2} \mathrm{O}_{3}-\mathrm{CNT}$ nanocomposite coating is discussed in the current work. Pretreatment of powder feedstock is required for dispersing CNTs in the matrix. Four coatings namely spray dried $\mathrm{Al}_{2} \mathrm{O}_{3}$ (A-SD), $\mathrm{Al}_{2} \mathrm{O}_{3}$ blended with 4 wt.\% CNT (A4C-B), composite spray dried $\mathrm{Al}_{2} \mathrm{O}_{3}-4$ wt.\% CNT (A4C-SD) and composite spray dried $\mathrm{Al}_{2} \mathrm{O}_{3}-8$ wt.\% CNT (A8CSD), are synthesized by plasma spraying. Owing to extreme temperatures and velocities involved in the plasma spraying of ceramics, retention of CNTs in the resulting coatings necessitates optimizing plasma processing parameters using an inflight particle diagnostic sensor. A bimodal microstructure was obtained in the matrix that consists of fully melted 
and resolidified structure and solid state sintered structure. CNTs are retained both in the fully melted region and solid-state sintered regions of processed coatings.

Fracture toughness of A-SD, A4C-B, A4C-SD and A8C-SD coatings was 3.22, 3.86, 4.60 and 5.04 $\mathrm{MPa} \mathrm{m}{ }^{1 / 2}$ respectively. This affirms the improvement of fracture toughness from $20 \%$ (in A4C-B coating) to $43 \%$ (in A4C-SD coating) when compared to the A-SD coating because of the CNT dispersion. Fracture toughness improvement from $43 \%$ (in $\mathrm{A} 4 \mathrm{C}-\mathrm{SD}$ ) to $57 \%$ (in $\mathrm{A} 8 \mathrm{C}-\mathrm{SD}$ ) coating is evinced because of the CNT content. Reinforcement by CNTs is described by its bridging, anchoring, hook formation, impact alignment, fusion with splat, and mesh formation.

The $\mathrm{Al}_{2} \mathrm{O}_{3} / \mathrm{CNT}$ interface is critical in assisting the stress transfer and utilizing excellent mechanical properties of CNTs. Mathematical and computational modeling using ab-initio principle is applied to understand the wetting behavior at the $\mathrm{Al}_{2} \mathrm{O}_{3} / \mathrm{CNT}$ interface. Contrasting storage modulus was obtained by nanoindentation $(\sim 210,250$, 250-350 and 325-420 GPa in A-SD, A4C-B, A4C-SD, and A8C-SD coatings respectively) depicting the toughening associated with CNT content and dispersion. 


\section{TABLE OF CONTENTS}

CHAPTER

PAGE

1. INTRODUCTION

1.1. Challenges in Fabricating CNT Reinforced Ceramic Nanocomposites

1.1.1. Dispersion of CNTs in the Ceramic Matrix

I.I.2. Retention of Undamaged CNTs in Plasma Sprayed Deposit

1.1.3. Grain Growth during Consolidation of Nanocrystalline Ceramic Matrix

1.2. Research Objectives

2. LITERATURE REVIEW

2.1. Need of Developing Tough Structural Ceramics 6

2.1.1. Non-Oxide Ceramics

2.1.2. Oxide Ceramics

2.2. Nature and Properties of Aluminum Oxide

2.3. Ceramics Processing via Thermal Spraying

2.3.1. Plasma Spraying

2.3.1.1. Plasma Sprayed Nanocrystalline Ceramic Coatings

2.3.1.2. Spray Drying of Ceramic Nanopowders

2.4. Toughening of Aluminum Oxide

2.5. Carbon Nanotubes as Reinforcement for Nanocomposite for Improving Mechanical Properties

2.5.1. CNT Reinforced Polymer Matrix Nanocomposites

2.5.2. CNT Reinforced Metal Matrix Nanocomposites

2.5.3. CNT Reinforced Ceramic Nanocomposites

2.6. Aluminum Oxide-CNT Nanocomposite

2.7. What's Missing and What's the Challenge?

3. EXPERIMENTAL PROCEDURE 62

3.1. Pretreatment of Powder Feedstock $\quad 62$

3.1.1. As Received $\mathrm{Al}_{2} \mathrm{O}_{3}$ Powder and CNTs 62

3.1.2. Spray Drying of As-Received $\mathrm{Al}_{2} \mathrm{O}_{3}$ Powder 63

3.1.3. Blending of Spray Dried $\mathrm{Al}_{2} \mathrm{O}_{3}$ with 4 wt. \% CNT 65

3.1.4. Spray Drying of Composite $\mathrm{Al}_{2} \mathrm{O}_{3}$ and CNT 65

3.2. Plasma Spraying of $\mathrm{Al}_{2} \mathrm{O}_{3}$ - CNT Nanocomposite Coating 67

3.3. Microstructural and Phase Characterization 75

3.3.1. Optical Microscopy and Quantitative Analysis of CNT Dispersion

3.3.2. Phase Analysis in Powder Feedstock and Plasma Sprayed Coatings 
3.3.3. Confirmation of CNT Retention by Raman Spectroscopy

3.3.4. Microstructural Characterization of $\mathrm{Al}_{2} \mathrm{O}_{3}-\mathrm{CNT}$ Nanocomposite

3.4. Mechanical Testing

3.4.1. Vicker Indentation Toughness

3.4.2. Nanoindentation

3.5. Ab-initio Molecular Modeling of Alumina-CNT Interface

4. RESULTS AND DISCUSSION

4.1. Powder Treatment of $n-\mathrm{Al}_{2} \mathrm{O}_{3}$ as Ideal Feedstock for Plasma

Spraying

4.2. Optimization of Plasma Processing Parameters

4.2.1. Optimization of Plasma Parameters on $n-\mathrm{Al}_{2} \mathrm{O}_{3}$ Coating

4.3. Plasma Spraying of $n-\mathrm{Al}_{2} \mathrm{O}_{3}$ with Addition of CNTs

4.3.1. Plasma Sprayed A4C-B Coating

4.3.2. Plasma Sprayed A4C-SD Coating

4.3.3. Plasma Sprayed A8C-SD Coating

4.4. Retention of CNTs in Plasma Sprayed of $\mathrm{Al}_{2} \mathrm{O}_{3}-\mathrm{CNT}$ Coatings

4.5. Effect of CNTs in Generating Microstructure and Phases in Plasma Sprayed Coatings

4.5.1. Role of CNT Dispersion in Creating Bimodal Grain

Distribution in Matrix

4.5.2. Generation of Metastable $\gamma-\mathrm{Al}_{2} \mathrm{O}_{3}$

4.5.2.1. Dependence of $\gamma-\mathrm{Al}_{2} \mathrm{O}_{3}$ on CNTs in the matrix

4.6. Role of CNT Dispersion in the Fracture Toughening of

Nanocomposite Ceramic Coatings

4.6.1. Enhancement of Fracture Toughness by CNT Distribution in A4C-B Coating

4.6.2. Enhancement of Fracture Toughness by CNT Dispersion in A4C-SD Coating

4.6.3. Enhancement of Fracture Toughness by CNT Dispersion in A8C-SD Coating

4.7. Wettability of $\mathrm{CNT}-\mathrm{Al}_{2} \mathrm{O}_{3}$ Interface

4.8. Ab-initio Computational Modeling of $\mathrm{CNT}-\mathrm{Al}_{2} \mathrm{O}_{3}$ Interface 168

4.9. Nanomechanical Modulus Mapping of Plasma Sprayed Coatings 174

4.9.1. Modulus Mapping of A-SD Coating

4.9.2. Modulus Mapping of A4C-B Coating

175

4.9.3. Modulus Mapping of A4C-SD Coating

4.9.4. Modulus Mapping of A8C-SD Coating

6. RECOMMENDATIONS FOR FUTURE 
6.2. Plasma Spraying of Insitu grown CNTs On $\mathrm{Al}_{2} \mathrm{O}_{3}$ Powder Particles

6.3. Molecular Dynamics Simulation of $\mathrm{Al}_{2} \mathrm{O}_{3} / C N T$ Interface

6.4. Hot-Isostatic Pressing of Plasma Sprayed Nanocomposites

6.5. Estimating Flexural Strength of Plasma Sprayed $\mathrm{Al}_{2} \mathrm{O}_{3}-\mathrm{CNT}$ Nanocomposite

6.6.Sliding Wear Evaluation of $\mathrm{Al}_{2} \mathrm{O}_{3}$ with $\mathrm{CNT}$ Addition

L1ST OF REFERENCES 


\section{LIST OF TABLES}

\section{TABLE}

Table 2.1: Fracture toughening of non-oxide structural ceramics

Table 2.2: Fracture toughness and toughening mechanisms for oxide ceramics

Table 2.3: Crystal structure, physical, thermal and mechanical properties of $\mathrm{Al}_{2} \mathrm{O}_{3}$

Table 2.4: Fabrication of ceramics by thermal spraying

Table 2.5: Role of additives in enhancing fracture toughness of $\mathrm{Al}_{2} \mathrm{O}_{3}$

Table 2.6: CNT-reinforced polymer nanocomposites

Table 2.7: Mechanical property enhancement in metal-CNT nanocomposite

Table 2.8: Evolution and development of CNT-reinforced ceramics by various processing techniques

Table 2.9: Description of ceramic-CNT nanocomposites research

Table 2.10: Description of $\mathrm{Al}_{2} \mathrm{O}_{3}-\mathrm{CNT}$ nanocomposites research

Table 2.11: Nomenclature for powders and hot-pressed $\mathrm{MgAl}_{2} \mathrm{O}_{4}$ samples

Table 3.1: Features of plasma spraying

Table 3.2: Representative processing parameters for plasma spraying

Table 4.1: Initial powder size, spray drying treatment and adopted nomenclature

Table 4.2: Toughening in micro- and nano-crystalline grains

Table 4.3: Plasma spraying parameters used for spraying $\mathrm{Al}_{2} \mathrm{O}_{3}$

Table 4.4: Microstructural features and fracture toughness of plasma sprayed $\mathrm{nAl}_{2} \mathrm{O}_{3}$ coatings 
Table 4.5: Plasma parameters for spraying A4C-B, A4C-SD and A8C-SD coating

Table 4.6: Inflight particle diagnostic data for various plasma sprayed coatings

Table 4.7: Volume percentage of microstructural features of the plasma sprayed coatings obtained from quantitative microscopy and image analysis

Table 4.8: Crystallite size and content of powder and plasma sprayed coatings

Table 4.9: Hardness and fracture toughness of plasma sprayed coatings

Table 4.10: Theoretical calculations of capillary force and surface tension on $\mathrm{Al}_{2} \mathrm{O}_{3}-\mathrm{CNT}$ interface (CNT diameter assumed to be $70 \mathrm{~nm}$ ) 


\section{LIST OF FIGURES}

FIGURE

Fig. 1.1: Summary of the work plan.

Fig. 2.1: Fracture toughness of $\mathrm{SiC}$ ceramics sintered with $\mathrm{Re}_{2} \mathrm{O}_{3}$ and AIN.

Fig. 2.2: HRTEM micrographs revealing clean interfaces without the amorphous inter granular phase in $\mathrm{SiC}-\mathrm{AlN}-\mathrm{Sc}_{2} \mathrm{O}_{3}:$ (a) $\mathrm{SiC}-\mathrm{SiC}$ boundary and (b) SiC-junction phase boundary.

Fig. 2.3: Comparison of a) nano- and macro-hardness and b) fracture toughness of $\mathrm{Si}_{3} \mathrm{~N}_{4} / \mathrm{SiC}$ nanocomposites.

Fig. 2.4: Vicker Indentation on WC ceramic depicting origination of radial cracks.

Fig. 2.5: SEM micrographs of the thermally etched surfaces of the $\mathrm{Y}-$ $\mathrm{ZrO}_{2}-\mathrm{ZrTiO}_{4}$ composites sintered at $1400{ }^{\circ} \mathrm{C}$ with a) 0 vol. $\%$, and b) $10 \mathrm{vol} . \% \mathrm{TiO}_{2}$.

Fig. 2.6: Vickers hardness and crack propagation resistance for coatings made from nanostructured and conventional $\mathrm{TiO}_{2}$ feedstock sprayed via HVOF and APS.

Fig. 2.7: Vickers indentation impression (1 kgf) in the cross-section of the HVOF-sprayed nanostructured $\mathrm{TiO}_{2}$ coating (a) and the indentation crack tip being arrested by a zone of nanostructured particles (b).

Fig. 2.8: Crystal Structure of $\mathrm{Al}_{2} \mathrm{O}_{3}(\mathrm{a}=\mathrm{b}=4.7564 \AA, \mathrm{c}=12.9894 \AA$, and $\alpha=\beta=90^{\circ}, \gamma=120^{\circ}$ ).

Fig. 2.9: (a) Change of stacking of $\{111\}$ spinel oxygen layers from fcc to hcp. (b) Shear of an oxygen layer having aluminum cations in the interstices. (c) Cooperative migration of the cations around regularly distributed vacancies, resulting in a "honey comb" lattice for $\alpha-\mathrm{Al}_{2} \mathrm{O}_{3}$.

Fig. 2.10: Schematic of plasma spraying process.

Fig. 2.11: Spray drying of ceramics. 
Fig. 2.12: Damping capacity vs dynamic modulus map of various thermally sprayed samples.

Fig. 2.13: TEM micrograph of plasma sprayed nano- $\mathrm{ZrO}_{2}$ coating layer.

Fig. 2.14: a) Secondary and b) Backscattered electron micrographs of plasma sprayed nano- $\mathrm{Al}_{2} \mathrm{O}_{3}-13 \mathrm{wt} . \% \mathrm{TiO}_{2}$ with additives. (a) Regions of splats (region $\mathrm{A}$ ) and small particulates (region $\mathrm{C}$ ), and (b) The backscattered electron image illustrates that the embedded particles appear darker than in the matrix.

Fig. 2.15: X-ray diffraction patterns from (113) $\alpha-\mathrm{Al}_{2} \mathrm{O}_{3}$ and (400) $\gamma-\mathrm{Al}_{2} \mathrm{O}_{3}$ peaks for reconstituted $\mathrm{Al}_{2} \mathrm{O}_{3}-13 \mathrm{wt} . \% \mathrm{TiO}_{2}$ with (modified) additives. The relative integrated intensity of these peaks are observed to vary as a function of CPSP.

Fig. 2.16: The relative density of the $\mathrm{Al}_{2} \mathrm{O}_{3}$ nanoceramics pressed at 1120 $\mathrm{MPa}$ and sintered at different temperatures for $5 \mathrm{~h}$ as a function of mean particle size of the starting $\alpha-\mathrm{Al}_{2} \mathrm{O}_{3}$ nanopowders.

Fig. 2.17: TEM micrograph of an $\mathrm{Al}_{2} \mathrm{O}_{3}-1 \mathrm{~mol} \% \mathrm{TiO}_{2}-1 \mathrm{~mol} \% \mathrm{MgO}$ solid solution sintered at $1500^{\circ} \mathrm{C}$ for $5 \mathrm{~h}$ in air.

Fig. 2.18: Insitu tensile elongation of individual single walled carbon nanotubes viewed under HRTEM. Tensile elongation under a-d) constant bias of $2.3 \mathrm{~V}$, and e-g) without bias. Vertical arrows show the kinks and horizontal arrows represent the kink movement direction in carbon nanotubes.

Fig. 2.19: TEM image of bent $\mathrm{CNT}$ with $\mathrm{A}$ and $\mathrm{B}$ ) radius of curvature $\sim 40$ $\mathrm{nm}$, and C) amplitude of ripples increase at the outer layer.

Fig. 2.20: CNT ropes are observed bridging a fatigue fracture surface in an epoxy matrix.

Fig. 2.21: Retention and CNT pullout is observed in the high magnification SEM image of fractured surface of Al- CNT composite.

Fig. 2.22: $\mathrm{SEM}$ of the fractured surface of $\mathrm{Al}_{2} \mathrm{O}_{3}-\mathrm{CNT}$ composite sintered at 48 $1450^{\circ} \mathrm{C}$ showing $\mathrm{CNT}$ pullout.

Fig. 2.23: SEM images of fracture profiles of the $\mathrm{CNT}-\mathrm{Fe}-\mathrm{Al}_{2} \mathrm{O}_{3}$ (a) and $\mathrm{CNT}-\mathrm{Fe} / \mathrm{Co}-\mathrm{MgAl}_{2} \mathrm{O}_{4}$ (b) composites prepared by extrusion at $1500^{\circ} \mathrm{C}$. Note the alignment of the CNTs. 
Fig. 2.24: Fracture toughness versus carbon nanotube volume content in aluminum oxide based composites as reported in literature. (SWCNT: Single walled CNT).

Fig. 2.25: TEM Micrographs of 5.7 vol. $\% \mathrm{SWCN} / \mathrm{Al}_{2} \mathrm{O}_{3}$ nanocomposite. (a) Bright-field TEM image and (b) high-resolution TEM image of specimens in the fully dense $5.7 \mathrm{vol} \% \mathrm{SWCN} / \mathrm{Al}_{2} \mathrm{O}_{3}$ nanocomposite. The arrows indicate the SWCN phase. (SWCNT: Single walled CNT).

Fig. 2.26: a) SEM photograph of a fracture surface of the $20 \mu \mathrm{m}$-thick sample demonstrating CNT pullout, longitudinal delamination, and residual holes; (b) crack deflection at CNT along interface.

Fig. 2.27: High resolution SEM images of the fracture surface of the hotpressed composites: (a) Sample without CNT showing mainly transgranular fracture. (b)-(e) Samples C5, C10, CM2 and CM5, respectively, showing CNT emerging out of the surface fracture. $\mathrm{C} 10$ and $\mathrm{CM} 2$ show restricted grain-growth effect (and intergranular fracture). (f) Sample CM12 showing many CNT emerging out of large pores and straightened before breaking during the fracture.

Fig. 2.28: The micrographs of carbon nanotube reinforced aluminum oxide composite powders. (a) The optical micrographs of dried gel and (b) the SEM micrographs of calcinated carbon nanotube/ aluminum oxide composite powders.

Fig. 2.29: A bird's view of $\mathrm{Al}_{2} \mathrm{O}_{3} / \mathrm{CNT}$ nanocomposite research efforts by various groups, their conclusions and remaining challenges.

Fig. 3.1: As-received $\mathrm{Al}_{2} \mathrm{O}_{3}$ powder particles.

Fig. 3.2: TEM of Multiwalled CNTs.

Fig. 3.3: a) Spray Dried $\mathrm{Al}_{2} \mathrm{O}_{3}$ powder, and b) Enlarged SEM image showing spray-dried agglomerated mass of fine powders.

Fig. 3.4: Process schematic and SEM images of a) nano $\mathrm{Al}_{2} \mathrm{O}_{3}$, b) spray dried $\mathrm{Al}_{2} \mathrm{O}_{3}$ (A-SD) agglomerates, and c) spray dried $\mathrm{Al}_{2} \mathrm{O}_{3}$ blended with 4 wt. \% CNT for $24 \mathrm{~h}$ to result nanocomposite $\mathrm{A} 4 \mathrm{C}$ B powder feedstock. 
Fig. 3.5: SEM image of A4C-SD powder showing a) powder agglomerates, b) dispersion of CNTs as separate entities, and c) fractured surface showing inside of the powder.

Fig. 3.6: Spray dried A8C-SD powder showing a) spherical agglomerates, and b) uniform CNT dispersion on surface.

Fig. 3.7: Fracture surface of $A 8 C$-SD powder indicating uniform $C N T$ dispersion in the core of the powder agglomerate.

Fig. 3.8: Schematic of plasma spraying process.

Fig. 3.9: Typical plasma sprayed coating showing uniform coating thickness.

Fig. 3.10:AccuraSpray: Inflight particle diagnostic sensor: (a) Controller with display monitor, (b) the sensor head, and (c) a screenshot showing temperature, velocity and plume profile.

Fig. 3.11: Detection of particle velocity from the time traveled within fixed distance between two optical fibers.

Fig. 4.1: XRD spectrum of as-received and spray dried $n-\mathrm{Al}_{2} \mathrm{O}_{3}$ powder indicating no change in phase.

Fig. 4.2: Raman spectrum showing similar peaks in the as-received and spray dried $n-\mathrm{Al}_{2} \mathrm{O}_{3}$ powder.

Fig. 4.3: a) Scattergram and representative micrograph describing CNT dispersion angle with respect to aspect ratio, and b) Histogram of A4C-B powder depicting agglomeration of CNTs in the dome cavity of the powder agglomerate.

Fig. 4.4: a) Scattergram and representative micrograph describing CNT dispersion angle with respect to aspect ratio, and b) Histogram of A4C-SD powder depicting dispersion angle with respect to the number of CNTs in the powder agglomerate.

Fig. 4.5: a) Scattergram and representative micrograph describing CNT dispersion angle with respect to aspect ratio, and b) Histogram of A8C-SD powder depicting dispersion angle with respect to the number of CNTs in the powder agglomerate. 
Fig. 4.6: Raman spectrum of the initial powder feedstock (before plasma spraying). D and G peaks correspond to defect and graphitic structure of CNT.

Fig. 4.7: Optimized plasma spraying to result surface melting and resolidification and core consolidation via solid state sintering.

Fig. 4.8: Plasma sprayed coatings (from top: Sample A, B, C, and D respectively) on an AISI 1020 steel coupon of size $100 \times 19 \times 3.1$ $\mathrm{mm}^{3}$. Coating thickness varies between $120-500 \mu \mathrm{m}$.

Fig. 4.9: Plasma spray parameter optimization showing relation of microstructural features of sprayed coatings with differential plasma parameter settings.

Fig. 4.10: Plasma sprayed $\mathrm{nAl}_{2} \mathrm{O}_{3}$ coating: a) Sample A, and b) Sample B, c) Sample C, and d) Sample D from A-SD powder.

Fig. 4.11: Plasma sprayed $\mathrm{nAl}_{2} \mathrm{O}_{3}$ coating (Sample A) showing a) PM, FM and porosity distribution, and $b$ ) solid-state sintering of powders. Note that grain size is around $1 \mu \mathrm{m}$.

Fig. 4.12: Plasma sprayed $\mathrm{nAl}_{2} \mathrm{O}_{3}$ coating (Sample B) showing a) PM, FM and porosity distribution, and $b$ ) solid-state sintering of powders. Note that grain size is decreased to around $0.8 \mu \mathrm{m}$.

Fig. 4.13: Plasma sprayed $\mathrm{nAl}_{2} \mathrm{O}_{3}$ coating (Sample C) showing a) PM, FM and porosity distribution, and $b$ ) solid-state sintering of powders. Note that grain size is further decreased to around $0.5 \mu \mathrm{m}$.

Fig. 4.14: Plasma sprayed $\mathrm{nAl}_{2} \mathrm{O}_{3}$ coating (Sample D) showing a) PM, FM and porosity distribution, and $b$ ) solid-state sintering of powders. Note that grain size is $\sim 0.35 \mu \mathrm{m}$.

Fig. 4.15: Radial crack generation in plasma sprayed $n-\mathrm{Al}_{2} \mathrm{O}_{3}$ coating via Vicker indentation.

Fig. 4.16: Plasma sprayed a) A-SD, b) A4C-B, c) A4C-SD, and d) A8C-SD coating.

Fig. 4.17: Cross-sectional image of A4C-B coating showing uniform and adherent coating.

Fig. 4.18: Plasma sprayed A4C-B coating showing fully melted (FM) and solid state sintered $(\mathrm{PM})$ region. 
Fig. 4.19: Fractured surface image of $\mathrm{A} 4 \mathrm{C}-\mathrm{B}$ coating depicting a) chain loop formation of CNTs, and b) fusion of CNTs in $\mathrm{Al}_{2} \mathrm{O}_{3}$.

Fig. 4.20: Cross-section of plasma sprayed A4C-SD coating.

Fig. 4.21: Plasma sprayed A4C-SD coating depicting enhanced PM content.

Fig. 4.22: a) Cross-section and b) fractured surface of plasma sprayed A4C-SD coating showing CNT retention and distribution.

Fig. 4.23: Cross-sectional SEM image of plasma sprayed A8C-SD coating.

Fig. 4.24: Plasma sprayed A8C-SD coating showing FM and PM regions.

Fig. 4.25: Solid state sintered region showing a) CNT retention in the plasma sprayed A8C-SD coating, and b) CNT distribution at neck, resolidified region and surface of $\mathrm{Al}_{2} \mathrm{O}_{3}$ particles.

Fig. 4.26: Density of plasma sprayed coatings measured from water immersion technique.

Fig. 4.27: Raman spectrum of plasma sprayed coatings showing retention of CNT structure in the plasma sprayed coatings.

Fig. 4.28: XRD spectrum of plasma sprayed coatings. Indicated peaks belong to $\gamma-\mathrm{Al}_{2} \mathrm{O}_{3}$ whereas all other peaks belong to $\alpha-\mathrm{Al}_{2} \mathrm{O}_{3}$.

Fig. 4. 29: TEM image showing a) $\gamma-\mathrm{Al}_{2} \mathrm{O}_{3}$ precipitation on $\mathrm{CNT}$ surface, and High resolution TEM image showing $\gamma-\mathrm{Al}_{2} \mathrm{O}_{3}$ lattice fringes corresponding to b) (311) plane (lattice spacing of $2.38 \AA$, and c) (023) plane (lattice spacing of $2.19 \AA$ ).

Fig. 4.30: Fracture toughness of plasma sprayed coatings

Fig. 4.31: Fibrous $\mathrm{CNT}$ fused onto $\mathrm{Al}_{2} \mathrm{O}_{3}$ surface anchoring the splats.

Fig. 4.32: Hook and loop formation of CNTs.

Fig. 4.33: Hook formation by entanglement of CNTs in A4C-B coating.

Fig. 4.34: Impact alignment of CNTs along the intersplat region in the plasma sprayed $\mathrm{A} 4 \mathrm{C}-\mathrm{B}$ Coating.

Fig. 4.35: Increased axial shear and absorption of energy by impact alignment of CNTs along splat interface. 
Fig. 4.36: Restraining of crack-damage by presence of CNTs transverse to crack propagation path.

Fig. 4.37: SEM image of nano $\mathrm{Al}_{2} \mathrm{O}_{3}$ particles sticking to $\mathrm{CNT}$ rope surface in AC-B Coating.

Fig. 4.38: a) TEM micrograph depicting non-preferred dispersion of $\mathrm{Al}_{2} \mathrm{O}_{3}$ particles onto CNT surface, and b) incomplete sintering of $\mathrm{Al}_{2} \mathrm{O}_{3}$ particles embedded in CNT network and CNT fusion is observed. SAD pattern is embedded along with.

Fig. 4.39: SAD ring pattern as indexed for major $\alpha-\mathrm{Al}_{2} \mathrm{O}_{3}$ phase.

Fig. 4.40: CNT retention and dispersion is observed in the PM, FM and nano- $\mathrm{Al}_{2} \mathrm{O}_{3}$ regions.

Fig. 4.41: Dispersion of CNTs in FM, PM and splat regions is observed in A4C-SD coating.

Fig. 4.42: Non-agglomeration of CNTs in the nano- $\mathrm{Al}_{2} \mathrm{O}_{3}$ particle matrix.

Fig. 4.43: Engulfing of $\mathrm{Al}_{2} \mathrm{O}_{3}$ particles by $\mathrm{CNTs}$. $\mathrm{Al}_{2} \mathrm{O}_{3}$ sintering is also observed in the SEM image.

Fig. 4.44: CNT bridge formation between two splats in A4C-SD coating.

Fig. 4.45: CNT mesh formation observed due to capillarity in the plasma sprayed A4C-SD coating.

Fig.4.46: a) TEM image of dispersed $\mathrm{CNT}$ in $\mathrm{Al}_{2} \mathrm{O}_{3}$ matrix showing $\mathrm{CNT}$ fusion with $\mathrm{Al}_{2} \mathrm{O}_{3} . \mathrm{Al}_{2} \mathrm{O}_{3}$ particle sintering is also observed. Embedded SAD pattern is enlarged, and $b$ ) dual crystalline phases are seen in the diffraction image.

Fig. 4.47: Vicker indentation crack in A4C-SD coating depicting: a) radial crack generation, and b) crack deflection and restraining by CNTs and crack.

Fig. 4.48: CNT distribution in the plasma sprayed A8C-SD coating.

Fig. 4.49: SEM image showing CNT retention and agglomeration in A8CSD coating. 
Fig. 4.50: Fracture toughening enhancement via presence of CNTs in solid state sintered region and its presence with nano $\mathrm{Al}_{2} \mathrm{O}_{3}$ particles. $\mathrm{CNT}$ agglomeration as $\mathrm{CNT}$ network is also observed.

Fig. 4.51: CNT fusion occurring in A8C-SD coating to result a rope structure.

Fig. 4.52: Schematic of torsional stiffening by CNTs.

Fig. 4.53: TEM micrograph of A8C-SD coating showing, a) $\mathrm{Al}_{2} \mathrm{O}_{3}$ sintering with embedded $\mathrm{SAD} \alpha-\mathrm{Al}_{2} \mathrm{O}_{3}$ pattern, and $\mathrm{b}$ ) $\mathrm{CNT}$ fusion and agglomeration with embedded SAD pattern of $\gamma-\mathrm{Al}_{2} \mathrm{O}_{3}$.

Fig. 4.54: $\mathrm{SAD}$ Diffraction pattern of $\gamma-\mathrm{Al}_{2} \mathrm{O}_{3}$ indexed as zone axis of $[1 \overline{1} \overline{2}]$.

Fig. 4.55: a) High energy at surface results from unbalanced atoms, and b) Wetting characteristic of droplet on a substrate.

Fig. 4.56: Theoretical representation of the CNT wetting by molten $\mathrm{Al}_{2} \mathrm{O}_{3}$.

Fig. 4.57: Rapid kinetics of plasma spraying demonstrating freezing of $\mathrm{Al}_{2} \mathrm{O}_{3}$ droplet before surface tension is overcome by gravity.

Fig. 4.58: Surface forces on $\mathrm{CNT}-\mathrm{Al}_{2} \mathrm{O}_{3}$ interface (CNT diameter assumed $70 \mathrm{~nm})$.

Fig. 4.59: Stage 1 showing surface tension dominated bridge structure.

Fig. 4.60: Stage 2 showing CNTs entrapped in $\mathrm{Al}_{2} \mathrm{O}_{3}$.

Fig. 4.61: Stage 3 showing capillarity dominated CNT-mesh.

Fig. 4.62: Phase stability diagram calculated from FactSage software. Activity of $\mathrm{Al}_{2} \mathrm{O}_{3}=1$, activity of carbon $=1$, and activity of $\mathrm{Al}_{4} \mathrm{C}_{3}(\mathrm{~s})=1.7429 \times 10^{-18}$.

Fig. 4.63: $\mathrm{Al}_{2} \mathrm{O}_{3}$-CNT system defined in ab-initio computational modeling.

Fig. 4.64: Isosurface contours of graphite layers at a) uninfluenced planes, and $b)$ near interface plane.

Fig. 4.65: Isosurface of a) aluminum surface-terminated alumina crystal, b) aluminum-carbon pseudo metallic bond interference at aluminaCNT interface. 
Fig. 4.66: Interfacial Shear at $\mathrm{Al}_{2} \mathrm{O}_{3}-\mathrm{CNT}$ interface.

Fig. 4.67: Dominance of surface tension in forming CNT Y-junction ropes.

Fig. 4.68: Plasma sprayed A-SD coating showing, a) Surface topography, b) Modulus map, c) Line scan distribution of storage modulus with distance, and d) Histogram of modulus distribution.

Fig. 4.69: Plasma sprayed A4C-B coating showing, a) Surface topography, b) Modulus mapping, c) Line scan distribution of storage modulus with distance, and d) Histogram of modulus distribution.

Fig. 4.70: Plasma sprayed A4C-SD coating showing, a) Surface topography, b) Modulus mapping, c) Distribution of storage modulus with distance, and d) Histogram of modulus distribution.

Fig. 4.71: Plasma sprayed A8C-SD coating showing, a) Surface topography, b) Modulus mapping, c) Line scan distribution of storage modulus with distance, and d) Histogram of modulus distribution. 


\section{LIST OF SYMBOLS}

\begin{tabular}{|c|c|}
\hline SYMBOL & : EXPANSION \\
\hline$c$ & : Radial Crack Length \\
\hline$f_{c}$ & : Capillarity Force \\
\hline$h_{m}$ & : Meniscus Height \\
\hline$k$ & : Stiffness \\
\hline$m$ & : Mass of Sensor \\
\hline$p$ & : Pressure \\
\hline$r$ & : Radius \\
\hline$r$ & : Radial Distance \\
\hline$t$ & : Time \\
\hline$t$ & : CNT Wall Thickness \\
\hline$t$ & : Crystallite Size \\
\hline$v$ & : Velocity \\
\hline$w$ & : Displacement Along Axial Direction \\
\hline$x$ & : Displacement \\
\hline$\dot{x}$ & : Rate of Change of Displacement \\
\hline$\ddot{x}$ & : Acceleration \\
\hline$A$ & : Area \\
\hline$A_{c}$ & : Contact Area of Nanoindenter Tip \\
\hline$B$ & : Full Width Half Maximum Broadening (radians) \\
\hline$C$ & : Damping of System \\
\hline$C_{2}$ & : constant $(1.4388 \mathrm{~cm} \cdot \mathrm{K})$ \\
\hline$D$ & : Distance \\
\hline$E$ & : Young's Modulus (390 GPa for $\mathrm{Al}_{2} \mathrm{O}_{3}$ ) \\
\hline$E^{\prime}$ & : Storage Modulus \\
\hline$E^{\prime \prime}$ & : Loss Modulus \\
\hline$F_{0}$ & : Sinusoidal Force \\
\hline
\end{tabular}




\begin{tabular}{|c|c|}
\hline$G$ & : Free Energy \\
\hline G & : Shear Modulus \\
\hline$H_{v}$ & : Vickers' Hardness \\
\hline$I_{v}$ & : Nucleation Frequency \\
\hline K & : Fracture Toughness $\left(\mathrm{MPa} \mathrm{m}^{1 / 2}\right)$ \\
\hline$K_{v}$ & : Kinetic Parameter \\
\hline$L$ & : Length of CNT \\
\hline$N$ & : Number of Walls in CNT \\
\hline$P$ & : Applied Load \\
\hline$R$ & : Ratio of Radiant Energy \\
\hline$T$ & : Surface Temperature of Radiating Body \\
\hline$T_{s}$ & : Surface Tension \\
\hline$V$ & : Volume \\
\hline$\alpha$ & : Rhombohedral $\mathrm{Al}_{2} \mathrm{O}_{3}$ (hcp) \\
\hline$\gamma$ & : Cubic $\mathrm{Al}_{2} \mathrm{O}_{3}(\mathrm{fcc})$ \\
\hline$\gamma$ & : Surface Energy \\
\hline$\gamma \cdot \cos \alpha$ & : Vertical Component of Surface Tension \\
\hline$\delta$ & : Tetragonal/Orthorhombic $\mathrm{Al}_{2} \mathrm{O}_{3}$ (fcc) \\
\hline$\varepsilon$ & : Spectral Emissivity \\
\hline$\eta$ & : Cubic $\mathrm{Al}_{2} \mathrm{O}_{3}(\mathrm{fcc})$ \\
\hline$\theta$ & : Monoclinic $\mathrm{Al}_{2} \mathrm{O}_{3}(\mathrm{fcc})$ \\
\hline$\theta$ & : Bragg Angle \\
\hline$\theta$ & : Torsion Angle \\
\hline$\theta$ & : Wetting Angle \\
\hline$\theta^{\prime}, \theta^{\prime}$ & : Monoclinic $\mathrm{Al}_{2} \mathrm{O}_{3}(\mathrm{hcp})$ \\
\hline$\kappa$ & : Orthorhombic $\mathrm{Al}_{2} \mathrm{O}_{3}$ (hcp) \\
\hline$\lambda$ & : Wavelength \\
\hline$\sigma$ & : Axial Stress \\
\hline$\tau$ & Shear Stress \\
\hline
\end{tabular}




$\begin{array}{ll}v & : \text { Poisson's Ratio } \\ \chi & : \text { Hexagonal } \mathrm{Al}_{2} \mathrm{O}_{3}(\mathrm{hcp}) \\ \phi & : \text { Phase Shift } \\ \chi & : \text { Material independent constant }(=0.016) \\ \omega & : \text { Frequency } \\ \Gamma & : \text { Surface Energy } \\ \Omega & : \text { Surface Projection Height }\end{array}$




\section{INTRODUCTION}

The goal of the proposed research is to improve fracture toughness of nanocrystalline aluminum oxide by addition and dispersion of multiwalled carbon nanotube (CNT) reinforcements using plasma spray technique. Fig 1.1 summarizes the overall research plan.

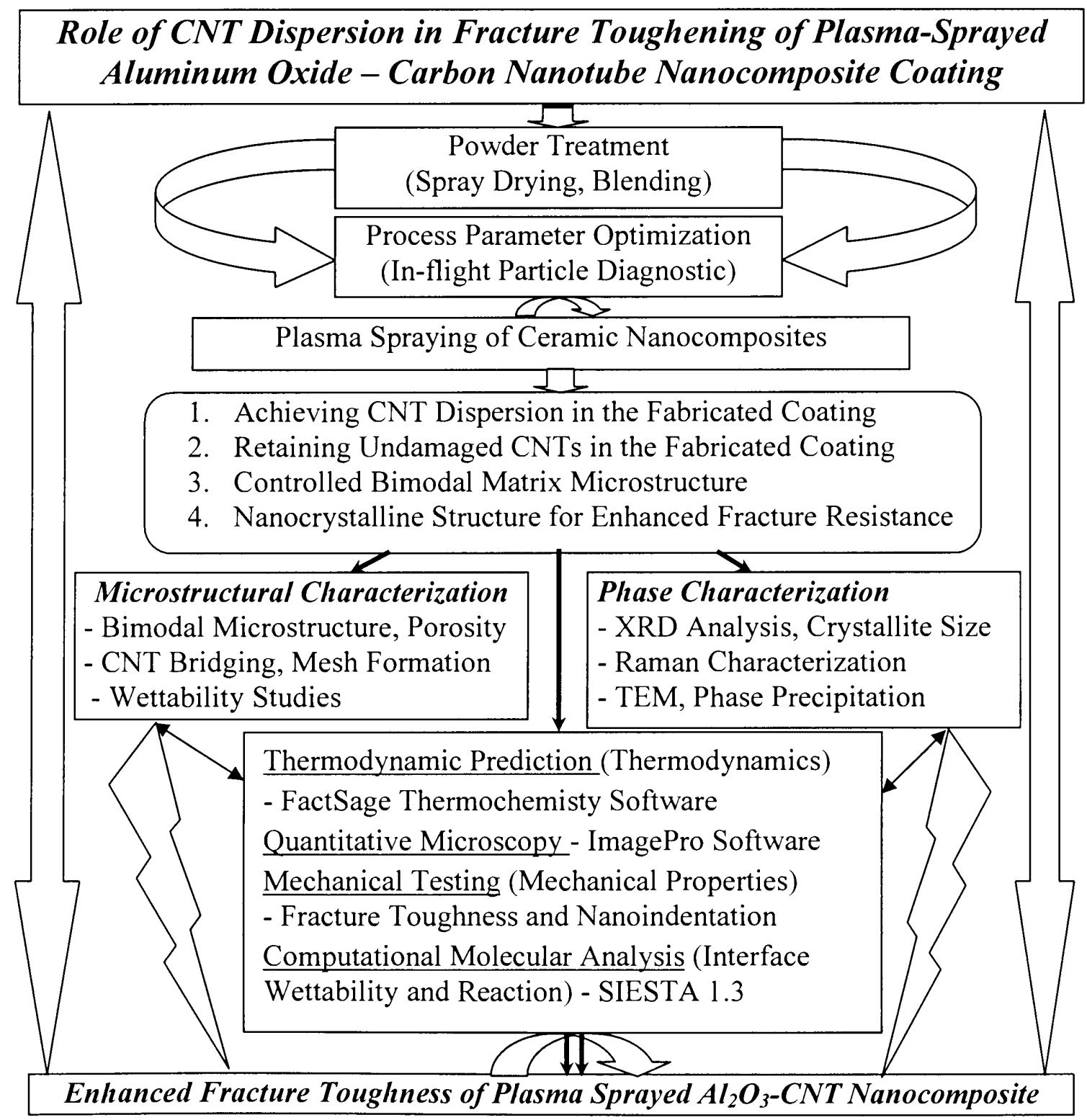

Fig. 1.1: Summary of the work plan 
Aluminum oxide (commercial name alumina, chemical formula $\mathrm{Al}_{2} \mathrm{O}_{3}$ ) is viewed as an ideal material for high temperatures application. However, the extremely brittle nature of $\mathrm{Al}_{2} \mathrm{O}_{3}$ ceramic limits its applications [1-14]. Hence there is a need to toughen $\mathrm{Al}_{2} \mathrm{O}_{3}$ for its use in structural applications. Due to their excellent mechanical properties, carbon nanotubes (CNT) has been identified as an ideal reinforcement to toughen the ceramics, including $\mathrm{Al}_{2} \mathrm{O}_{3}[9,15-31]$. Thermal spray processing of ceramic has accomplished superior coatings with improved mechanical properties such as indentation crack resistance, spallation resistance and enhanced fracture toughness [3, 32-36]. Therefore, the plasma spraying technique has been adopted in synthesizing $\mathrm{Al}_{2} \mathrm{O}_{3}-\mathrm{CNT}$ nanocomposite coating with enhanced fracture toughness. But processing and synthesis of CNT reinforced ceramic nanocomposite coating involves several challenges as summarized below:

\subsection{Challenges in Fabricating CNT Reinforced Ceramic Nanocomposites}

\subsubsection{Dispersion of CNTs in the Ceramic Matrix}

CNTs have a strong tendency to agglomerate owing to the high surfacearea/volume ratio. Dispersion of CNTs offer advantages of excellent mechanical properties since individual CNTs possess such as very high Young's modulus $(\sim 1 \mathrm{TPa})$, and fracture strength $(\sim 50 \mathrm{GPa})$ in enhancing the fracture toughness of ceramic nanocomposites $[11,22,25,37,38]$. Owing to layer by layer deposition in the plasma spraying, CNT homogeneity in the coatings can be achieved by dispersion of CNTs in the powder feedstock and subsequent coating process. Hence, powder feedstock pretreatment becomes a requirement for dispersing CNTs in the $\mathrm{Al}_{2} \mathrm{O}_{3}$ matrix. In the present study, the 
challenge of dispersing CNTs has been addressed by spray drying of composite ceramic and $\mathrm{CNT}$ powder. Consequently, plasma spraying should be optimized in order to retain CNTs in the sprayed coatings.

\subsubsection{Retention of Undamaged CNTs in Plasma Sprayed Deposit}

Particles passing through thermal plasma plume experience very high temperatures in excess of 10,000 Kelvin $[1,39-41]$. In addition, the exiting powder particles attain high velocity from sonic to supersonic regime $[1,39,41]$. Hence retention of undamaged CNTs at such extreme temperatures and impact is quite a challenging problem. Though earlier works have demonstrated successful retention of CNTs in the plasma sprayed metal matrix nanocomposite, ceramic matrix requires enhanced enthalpy plasma parameters which might damage CNTs during processing [42-44]. This challenge has been met by monitoring and controlling plasma parameters of processing via in-flight diagnostics. Owing to rapid solidification behavior of the plasma spraying and short flight time, undamaged CNTs can be retained in the nanocomposite coating.

\subsubsection{Grain Growth du ring Consolidation of Nanocrystalline Ceramic Matrix:}

Nanostructure enhances the strength and fracture toughness of the material. However, conventional consolidation techniques result in the grain growth of the nano particles $[2,5,8-10,17,21-31,45-52]$. Hence, retention of nanocrystalline structure after consolidation remains a hurdle to overcome. This has been achieved by controlled melting/heating of powder particles that require suitable processing protocol and 
optimized consolidation mechanism towards retaining nanostructure. High cooling rates which are inherent to the plasma spraying also aids in retention of nanostructure.

\subsection{Research Objectives}

The overall objective of current research is to elicit the role of CNT dispersion in the fracture toughening of plasma-sprayed aluminum oxide - carbon nanotube ceramic nanocomposite coating.

The specific research objectives of this work can be summarized as:

- Incorporation and retention of CNT to improve toughness of $\mathrm{Al}_{2} \mathrm{O}_{3}-\mathrm{CNT}$ nanocomposite structure via plasma spray forming.

- Dispersion of undamaged $\mathrm{CNT}$ in the $\mathrm{Al}_{2} \mathrm{O}_{3}$ matrix.

- Retention/refinement of nanostructure in $\mathrm{Al}_{2} \mathrm{O}_{3}$ matrix towards improving fracture toughness.

- Evaluation of bulk mechanical properties such as fracture toughness and elastic modulus of plasma sprayed coating.

- In-depth analysis of interfacial phenomenon and wetting characteristics between $\mathrm{Al}_{2} \mathrm{O}_{3}$ matrix and $\mathrm{CNT}$ reinforcement using experimental technique and molecular modeling of interface via SIESTA 1.3 simulation software.

This dissertation is systematically structured in separate chapters to thoroughly present the background, execution of ideas, assembling of results, scientific interpretation and future scope of the work. Chapter 2 describes the systematic progression of work done by various researchers in extending the scientific knowledge. Adoption of various 
techniques and ideas for achieving enhanced fracture toughness is reviewed in this chapter. Chapter 3 describes the methodologies of experiments and analysis adopted in the current work. The heart of the dissertation is the results and discussion, chapter 4 , where the toughening enhancement of $\mathrm{Al}_{2} \mathrm{O}_{3}$ by CNTs is presented and discussed in detail. Conclusions of the research are stated in chapter 5 summarizing the key findings and important feats. Chapter 6 states the recommendation for future work, which seem potential measures in continued improvement of the current research. 


\section{LITERATURE REVIEW}

\subsection{Need of Developing Tough Structural Ceramics}

Whether it be structural ceramics or ultra high temperature ceramics for reentry space vehicles, the very competence of ceramics requires: high refractoriness, chemical inertness, resistance to wear, good oxidation resistance, low coefficient of thermal expansion, high thermal conductivity, and good creep properties [1]. But, inherent brittleness of ceramics have created a need for enhancing the fracture toughness and flexural bend strength of the ceramics $[1,3,4,12,49,53,54]$. The structural ceramics can be broadly classified into non-oxide and oxide ceramics.

\subsubsection{Non-Oxide Ceramics}

Non-oxide ceramics constitute the borides, carbides, nitrides, and silicides. Ultrahigh temperature ceramics such as $\mathrm{HfC}, \mathrm{TaC}, \mathrm{ZrB}_{2}, \mathrm{BN}, \mathrm{HfN}, \mathrm{TiN} / \mathrm{TiB}_{2}$ and their composites have been used for rocket science and engineering applications that require structural integrity at temperature $>2100 \mathrm{~K}$ [55]. Toughening in the non-oxide ceramics has been achieved by grain refinement, densification by HlPping (hot-isostatic pressing) and crack-healing agents (such as glassy phase, or presence of microporosity) [8, 48]. A summary of strengthening and toughening mechanisms in non-oxide structural ceramics is presented in Table 2.1. It can be observed that most of the research work and 
commercial development of the non-oxide ceramics has been limited to $\mathrm{SiC}$ and $\mathrm{Si}_{3} \mathrm{~N}_{4}$ $[45,48,50,56]$.

Table 2.1: Fracture toughening of non-oxide structural ceramics

\begin{tabular}{|c|c|c|c|c|}
\hline Composition & $\begin{array}{l}\text { Toughening } \\
\text { Mechanism }\end{array}$ & $\begin{array}{l}\text { Fracture } \\
\text { Toughness }\end{array}$ & $\begin{array}{c}\text { Other features/ } \\
\text { Comments }\end{array}$ & Reference \\
\hline SiAlON-SiC & $\begin{array}{l}\text { Liquid phase } \\
\text { sintering }\end{array}$ & $\sim 6.0 \mathrm{MPa} \mathrm{m}^{1 / 2}$ & $\begin{array}{l}\text { Fine equiaxed } \\
\text { grains and dense } \\
\text { structure }\end{array}$ & [45] \\
\hline $\begin{array}{l}\mathrm{SiC}+\mathrm{AlN}+ \\
\text { Rare earths }\end{array}$ & $\begin{array}{c}\text { Smaller rare } \\
\text { earth cations } \\
\text { resulting clean } \\
\text { boundaries }\end{array}$ & $\sim 6.5 \mathrm{MPa} \mathrm{m}^{1 / 2}$ & $\begin{array}{l}\text { Composition and } \\
\text { microstructure of } \\
\text { sintering additives } \\
\text { affect toughness }\end{array}$ & {$[56]$} \\
\hline $\begin{array}{c}\text { WC- } 0.5 \mathrm{wt} \\
\% \mathrm{Co}-0.25 \\
\text { wt } \% \text { VC }\end{array}$ & $\begin{array}{l}\text { Abnormal grain } \\
\text { growth } \\
\text { reinforcement }\end{array}$ & $7.34 \mathrm{MPa} \mathrm{m}^{1 / 2}$ & $\begin{array}{l}\text { Toughening by } \\
\text { crack-cutting } \\
\text { elongated grains }\end{array}$ & [5] \\
\hline $\begin{array}{c}\mathrm{Si}_{3} \mathrm{~N}_{4} / \mathrm{SiC} \\
\left(5 \text { wt\% } \% \mathrm{Y}_{2} \mathrm{O}_{3}\right. \\
\text { with upto } 13 \\
\text { wt.\% } \% \mathrm{SiC})\end{array}$ & $\begin{array}{l}\text { Inter and intra } \\
\mathrm{SiC} \text { nano } \\
\text { inclusions }\end{array}$ & $\begin{array}{c}5.8 \mathrm{MPa} \mathrm{m}^{1 / 2} \\
(\text { at } 8 \mathrm{wt} . \% \mathrm{SiC} \text { ) }\end{array}$ & $\begin{array}{l}\text { Hardness } \\
\text { increased with } \\
\text { increase in } \mathrm{SiC} \\
\text { content }\end{array}$ & [50] \\
\hline $\mathrm{ZrB}_{2}-\mathrm{MoSi}_{2}$ & $\begin{array}{l}\text { Uniform and fine } \\
\text { microstructure }\end{array}$ & $\sim 2.6 \mathrm{MPa} \mathrm{m}^{1 / 2}$ & $\begin{array}{c}\text { Flexural strength } \\
\sim 655 \mathrm{MPa} \text { and } \\
500 \mathrm{MPa} \text { at } 1200 \\
\text { and } 1500{ }^{0} \mathrm{C} \\
\text { respectively. }\end{array}$ & [10] \\
\hline $\mathrm{C} / \mathrm{SiC}$ & $\begin{array}{c}\text { Presence of } \\
\text { micro cracks, } \\
\text { carbon fiber } \\
\text { pullout, } \\
\text { homogeneity and } \\
\text { non- } \\
\text { decomposition of } \\
\text { matrix }\end{array}$ & -- & $\begin{array}{c}\text { Flexural strength } \\
\sim 124-287 \mathrm{MPa}\end{array}$ & {$[57,58]$} \\
\hline
\end{tabular}

Composite of $\mathrm{Si}_{3} \mathrm{~N}_{4}$ with inter and intra $\mathrm{SiC}$ inclusions in wood-cutting ceramics provided enhancement of mechanical properties (fracture toughness increase from 5.1 to 5.8 $\mathrm{MPa} \mathrm{m}{ }^{1 / 2}$ and microhardness increase from $16 \mathrm{GPa}$ to $19 \mathrm{GPa}$ ) [50]. In addition, 
pressing and sintering techniques (thermal/pressureless/ liquid-phase) to manipulate microstructure in $\mathrm{ZrB}_{2}-\mathrm{MoSi}_{2}, \mathrm{SiC}, \mathrm{SiAlON}-\mathrm{SiC}$ ceramics have proved to be significant in enunciating toughening of ceramic composites $[10,45,56]$. The role of utilizing abnormal grain growth in $\mathrm{WC}$, and development of tough ternary carbide phase ceramics were next big feats in the field of non-oxide ceramic $[2,5,8,10,45-50]$. C/SiC composites fabricated by chemical vapor deposition/infiltration and sol-gel technique have emerged for applications such as aircraft brakes, and re-entry shields, rocket nozzles etc $[57,58]$.

Figure 2.1 demonstrates change of fracture toughness during sintering with addition of rare earth oxides in $\mathrm{SiC}$ matrix [56]. Clean interphase boundaries without amorphous inter granular phase, as observed in Fig. 2.2, is resulted by the addition of smaller rare-earth cations [56]. Composition and microstructure of the sintering additives strongly aid the toughening and strengthening of $\mathrm{SiC}$ ceramics. Figure $2.3 \mathrm{a}$ features increase of hardness of $\mathrm{Si}_{3} \mathrm{~N}_{4} / \mathrm{SiC}$ nanocomposite with increase in $\mathrm{SiC}$ content [50]. Apart from increasing $\mathrm{SiC}$ content, microstructure refinement hinders dislocation movement within $\mathrm{Si}_{3} \mathrm{~N}_{4}$ matrix to enhance hardness. Liquid phase sintering resulted percolation of $\mathrm{SiC}$ nano inclusions in the intergranular regions enhancing the fracture toughness, Fig. $2.3 b$. 


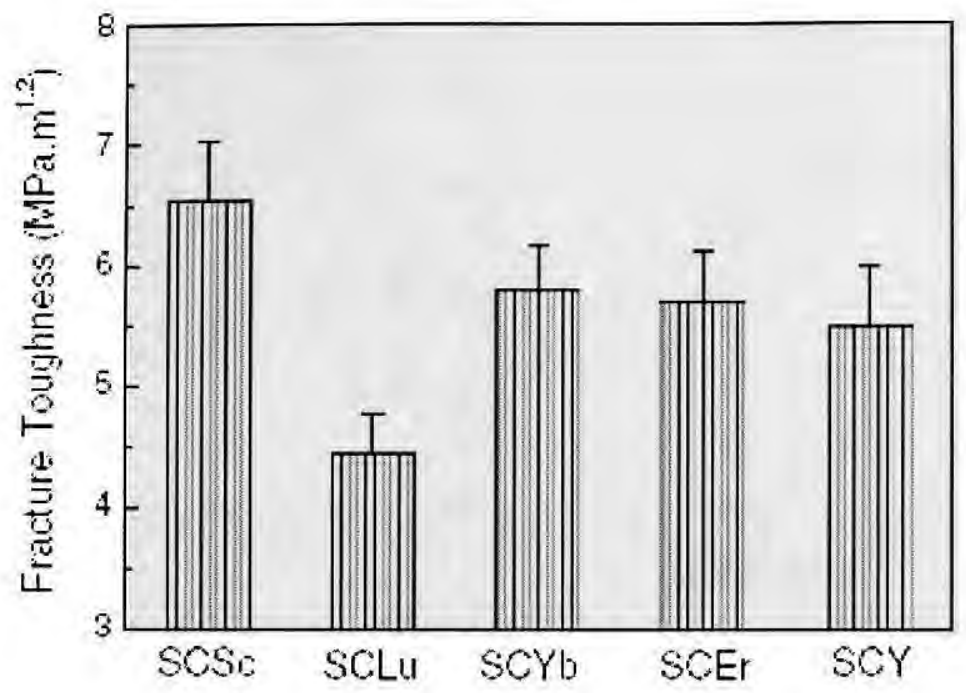

Fig. 2.1: Fracture toughness of $\mathrm{SiC}$ ceramics sintered with $\mathrm{Re}_{2} \mathrm{O}_{3}$ and $\mathrm{AlN}$ [56].

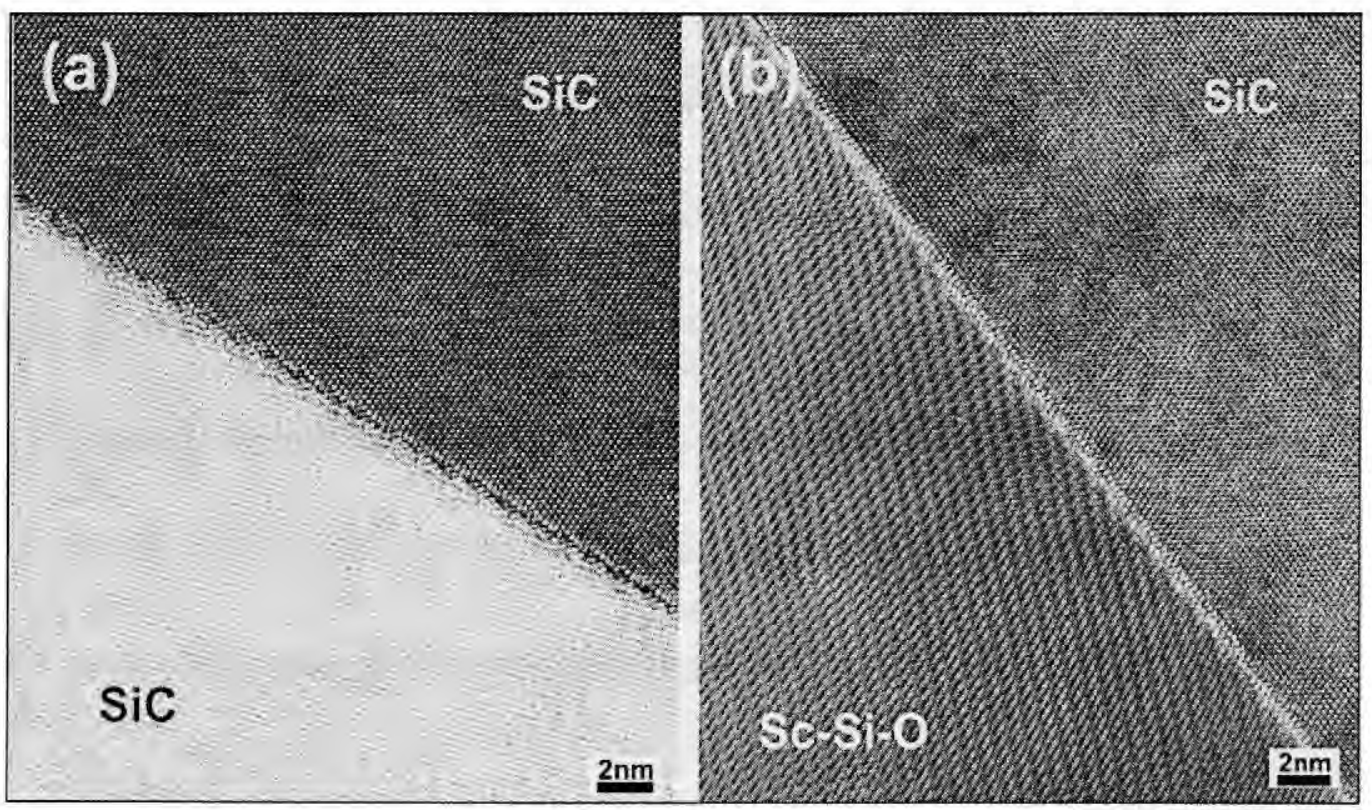

Fig. 2.2: HRTEM micrographs revealing clean interfaces without the amorphous inter granular phase in $\mathrm{SiC}-\mathrm{AlN}-\mathrm{Sc}_{2} \mathrm{O}_{3}$ : (a) $\mathrm{SiC}-\mathrm{SiC}$ boundary and (b) $\mathrm{SiC}$-junction phase boundary [56]. 

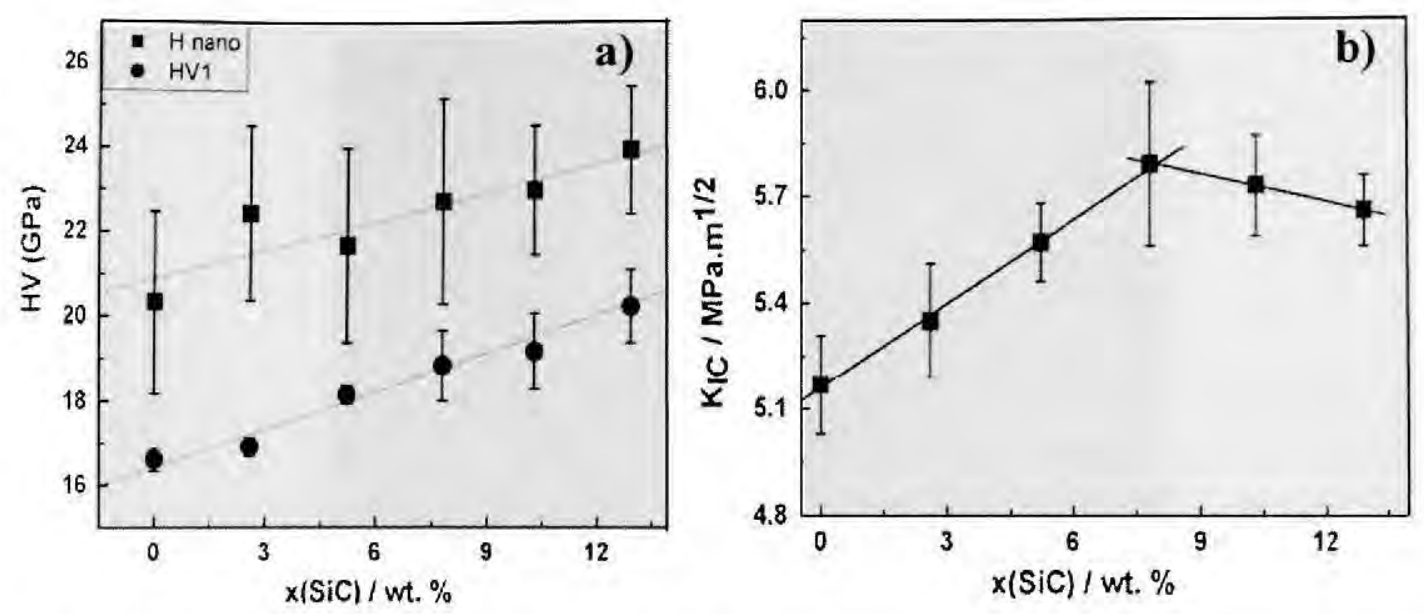

Fig. 2.3: Comparison of a) nano- and macro-hardness and b) fracture toughness of $\mathrm{Si}_{3} \mathrm{~N}_{4} / \mathrm{SiC}$ nanocomposites [50].

Figure 2.4 shows Vicker indentation generating radial crack in cobalt bonded WC. Vicker-crack generate due to residual stress relief upon unloading and the balancing relation of stress intensity ahead of crack tip with the crack-termination can indirectly help evaluating the fracture toughness of the material. When the Young's modulus for ceramics is known (taken as $390 \mathrm{GPa}$ for bulk $\mathrm{Al}_{2} \mathrm{O}_{3}$ ), indentation fracture toughness $(K)$ can be calculated by the semi-empirical formula given by Antis, equation 2.1 [59]:

$$
K=\chi\left(\frac{E}{H_{V}}\right)^{1 / 2}\left(\frac{P}{c}\right)^{3 / 2}
$$

where $\chi=0.016$ is material independent constant, $E$ is the Young's modulus, $H_{V}$ is the Vicker's hardness, $P$ is the applied load and $c$ is the crack length. 


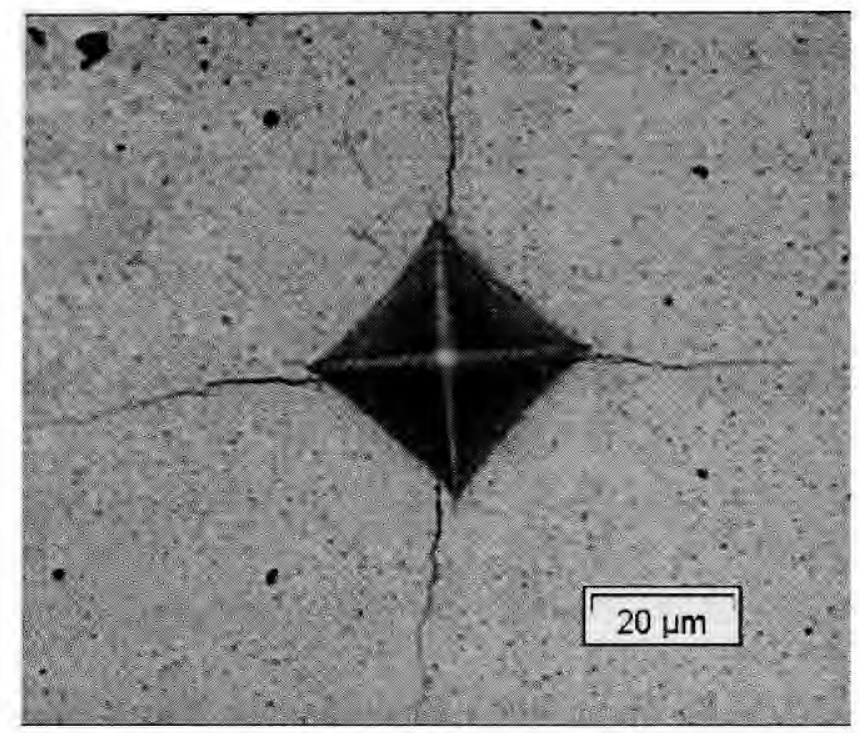

Fig. 2.4: Vicker Indentation on WC ceramic depicting origination of radial cracks [5].

Oxide ceramics and their toughening mechanism are reviewed in the next section.

\subsubsection{Oxide Ceramics}

Among ceramics, the oxides are most useful and common owing to ease of fabrication and stability at high temperatures than nitrides, carbides, sulfides and borides $[60,61]$. Oxide ceramics, such as $\mathrm{Al}_{2} \mathrm{O}_{3}, \mathrm{ZrO}_{2}, \mathrm{TiO}_{2}, \mathrm{Cr}_{2} \mathrm{O}_{3}, \mathrm{SiO}_{2}$, and $\mathrm{Y}_{2} \mathrm{O}_{3}$ offer advanced technological applications owing to high hardness, resistance to corrosion, and high refractoriness apart from high wear-, fretting-, cavitation- and erosion-resistance, and high dynamic modulus $[3,13,33,34,62]$. Though non-oxide ceramics display unique properties as potential candidates for extreme environments, they often require a protective oxide layer to create diffusion barrier for oxidation protection [60,63, 64]. 
Oxidation resistance becomes a natural requirement for the materials operating at elevated temperature in air. Aptly oxide ceramics emerge as oxidation-resistant structural materials $[60,64,65]$. High temperature applications introduce large volumetric phase transformations leading to the structural instability. And, inherent brittleness of the ceramic oxides makes them more susceptible to thermal shock failures. Thereby, toughening of the oxide ceramics becomes a prerequisite in their structural applications.

Conventionally, fracture toughening enhancement in oxides has been achieved through phase transformation or by introducing controlled/graded microstructure $[18,66-$ 68]. Incorporation of secondary phases in restricting crack propagation and nanocrystalline structure in enhancing grain sliding has also been prominent in improving the fracture toughness of ceramic nanocomposites $[6,36,69,70]$. Table 2.2 features fracture toughening mechanisms in oxide ceramics.

Specific examples of $\mathrm{Y}-\mathrm{ZrO}_{2}$ and $\mathrm{TiO}_{2}$ are considered to elicit toughening mechanisms in oxide ceramics. Initial particle size of $\mathrm{Y}-\mathrm{ZrO}_{2}$ was $\sim 50-200 \mathrm{~nm}$, whereas addition of fine $\mathrm{TiO}_{2}$ was selected in range of 100-300 nm. Fig. 2.5a shows pressed and sintered (at $1400^{\circ} \mathrm{C}$ for $4 \mathrm{~h}$ ) $\mathrm{Y}-\mathrm{ZrO}_{2}$ composite [66]. Due to agglomeration tendency of $\mathrm{TiO}_{2}$ particles, addition of 10 vol. $\% \mathrm{TiO}_{2}$, Fig. $2.5 \mathrm{~b}$, resulted increase in inhomogeneity and porosity of the nanocomposite. Increase in fracture toughness with addition of $10 \mathrm{vol}$. 
$\% \mathrm{TiO}_{2}$ was attributed to nucleation of $\mathrm{Y}-\mathrm{ZrO}_{2}-\mathrm{ZrTiO}_{4}$ with more pronounced transformation toughening when compared to without the $\mathrm{TiO}_{2}$ addition [66].

Table 2.2: Fracture toughness and toughening mechanisms for oxide ceramics

\begin{tabular}{|c|c|c|c|c|}
\hline $\begin{array}{l}\text { Ceramic } \\
\text { Oxide }\end{array}$ & $\begin{array}{l}\text { Toughening } \\
\text { Mechanism }\end{array}$ & $\begin{array}{l}\text { Fracture } \\
\text { Toughness }\end{array}$ & $\begin{array}{l}\text { Other features/ } \\
\text { Comments }\end{array}$ & Reference \\
\hline $\begin{array}{l}\text { YSZ (Yttria } \\
\text { stabilized } \\
\text { tetragonal } \\
\text { zirconia) }\end{array}$ & $\begin{array}{l}\text { Transformation } \\
\text { toughening } \\
\text { (tetragonal to } \\
\text { monoclinic } \mathrm{ZrO}_{2} \text { ) }\end{array}$ & $4.5 \mathrm{MPa} \mathrm{m}^{1 / 2}$ & $\begin{array}{l}\text { Additional } \\
\text { toughening by } \\
\mathrm{ZrTiO}_{4} \text { phase }\end{array}$ & [66] \\
\hline Nano- $\mathrm{TiO}_{2}$ & $\begin{array}{c}\text { Crack arrest by } \\
\text { nanostructured } \\
\text { zones }\end{array}$ & $\sim 27 \mathrm{MPa} \mathrm{m}^{1 / 2}$ & $\begin{array}{l}\text { Isotropic crack } \\
\text { propagation, and } \\
\text { distribution of } \\
\text { agglomerated } \\
\text { nanoparticles }\end{array}$ & {$[6]$} \\
\hline $\mathrm{SiO}_{2}$ & $\begin{array}{l}\text { Restricting } \\
\text { cristobalite by } \\
\text { AIN particles }\end{array}$ & $\begin{array}{l}\text { Upto } 2.96 \mathrm{MPa} \\
\mathrm{m}^{1 / 2} \text { at } 1400{ }^{\circ} \mathrm{C}\end{array}$ & $\begin{array}{l}\text { Microcrack } \\
\text { deflection and } \\
\text { divergence }\end{array}$ & [71] \\
\hline $\mathrm{MgAl}_{2} \mathrm{O}_{4}$ & $\begin{array}{l}\text { Restraining the } \\
\text { grain growth by } \\
\text { nano } \mathrm{Al}_{2} \mathrm{O}_{3}\end{array}$ & $7.79 \mathrm{MPa} \mathrm{m}^{1 / 2}$ & $\begin{array}{c}\text { Low temperature } \\
\text { diffusional creep, } \\
\text { thermally } \\
\text { activated } \\
\text { deformation }\end{array}$ & [69] \\
\hline $\mathrm{ZrO}_{2}$ & $\begin{array}{l}\text { Transformation } \\
\text { toughening }\end{array}$ & $10.1 \mathrm{MPa} \mathrm{m}^{1 / 2}$ & $\begin{array}{l}\text { Addition of } \\
\text { secondary } \\
\text { toughening by } \\
\text { WC, crack } \\
\text { deflection }\end{array}$ & [67] \\
\hline
\end{tabular}




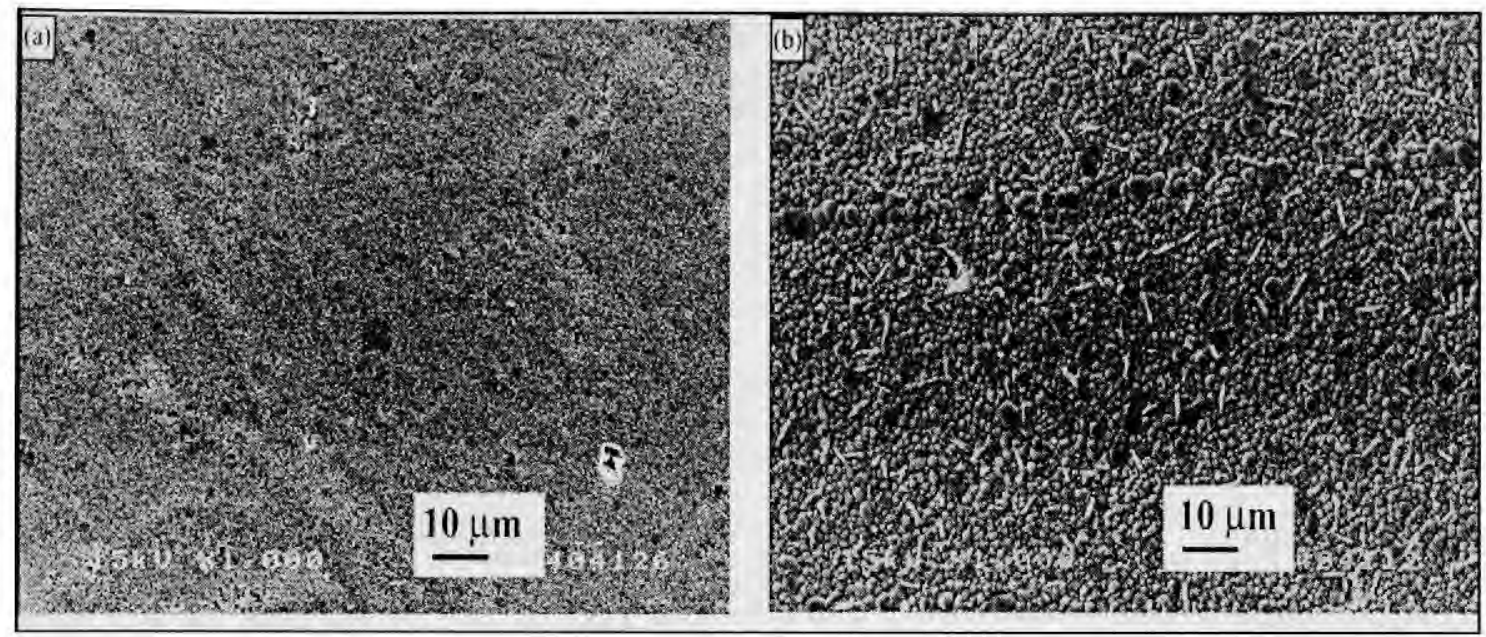

Fig. 2.5: SEM micrographs of the thermally etched surfaces of the $\mathrm{Y}-\mathrm{ZrO}_{2}-\mathrm{ZrTiO}_{4}$ composites sintered at $1400^{\circ} \mathrm{C}$ with a) 0 vol. $\%$, and b) 10 vol. $\% \mathrm{TiO}_{2}[66]$.

Fig. 2.6 shows hardness and crack propagation resistance of HVOF (high velocity oxy-fuel sprayed) and APS (air plasma sprayed) $\mathrm{TiO}_{2}$ coatings. Though hardness of all three processed coatings (viz. air plasma and HVOF sprayed $\mathrm{TiO}_{2}$, and HVOF sprayed nano $\mathrm{TiO}_{2}$ ) is similar, nano $\mathrm{TiO}_{2}$ demonstrated extremely high fracture toughness when compared to conventional processing, Fig. 2.6 (also see Table 2.2) [6]. Enhanced fracture toughening was attributed to arrest of cracks by nano-structured zones as observed in Fig. 2.7 [6]. Nanozones impede the crack path and restrict the crack extension by absorbing the crack-propagation energy and arresting the crack to result enhanced fracture toughness. 


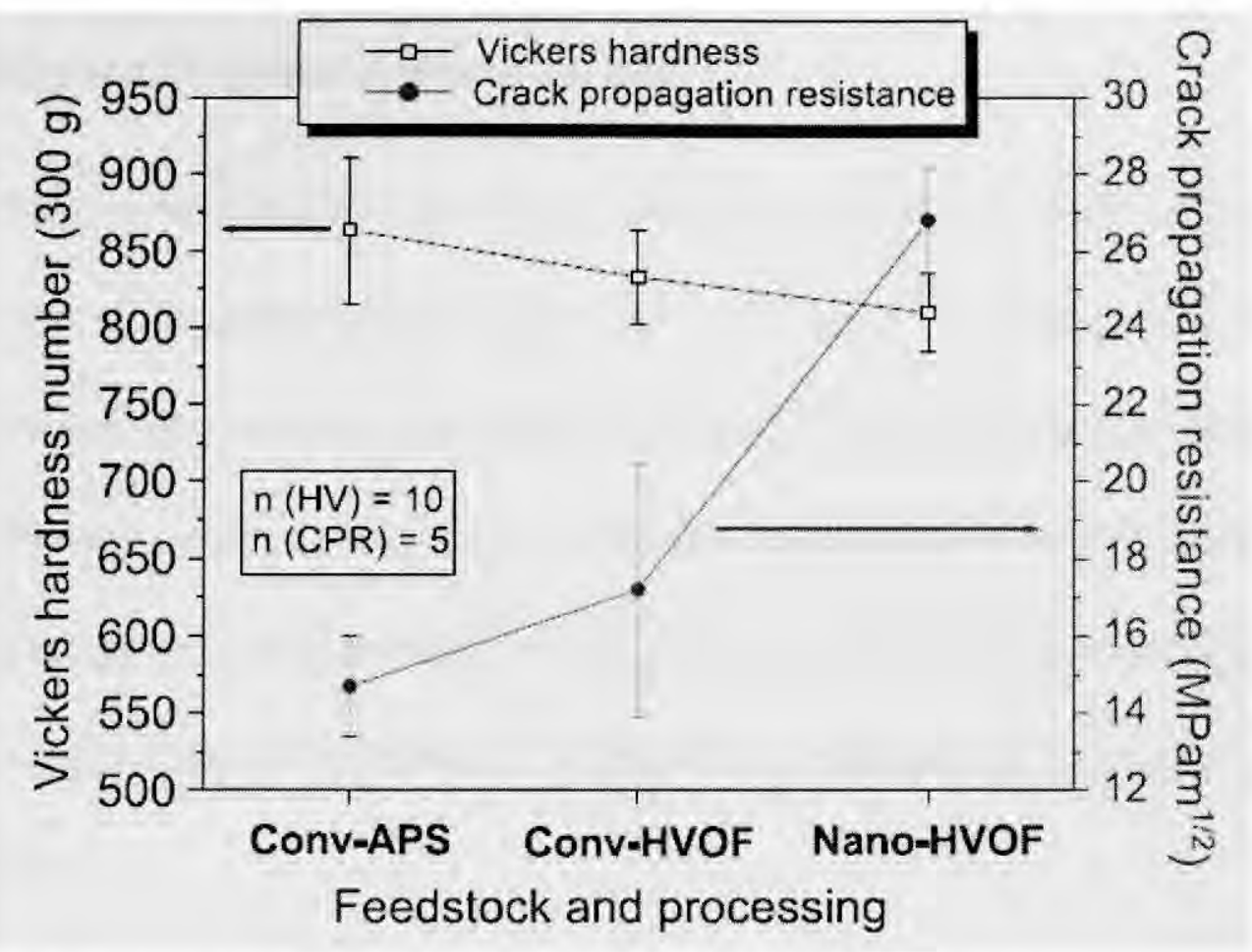

Fig. 2.6: Vickers hardness and crack propagation resistance for coatings made from nanostructured and conventional $\mathrm{TiO}_{2}$ feedstock sprayed via HVOF and APS [6].
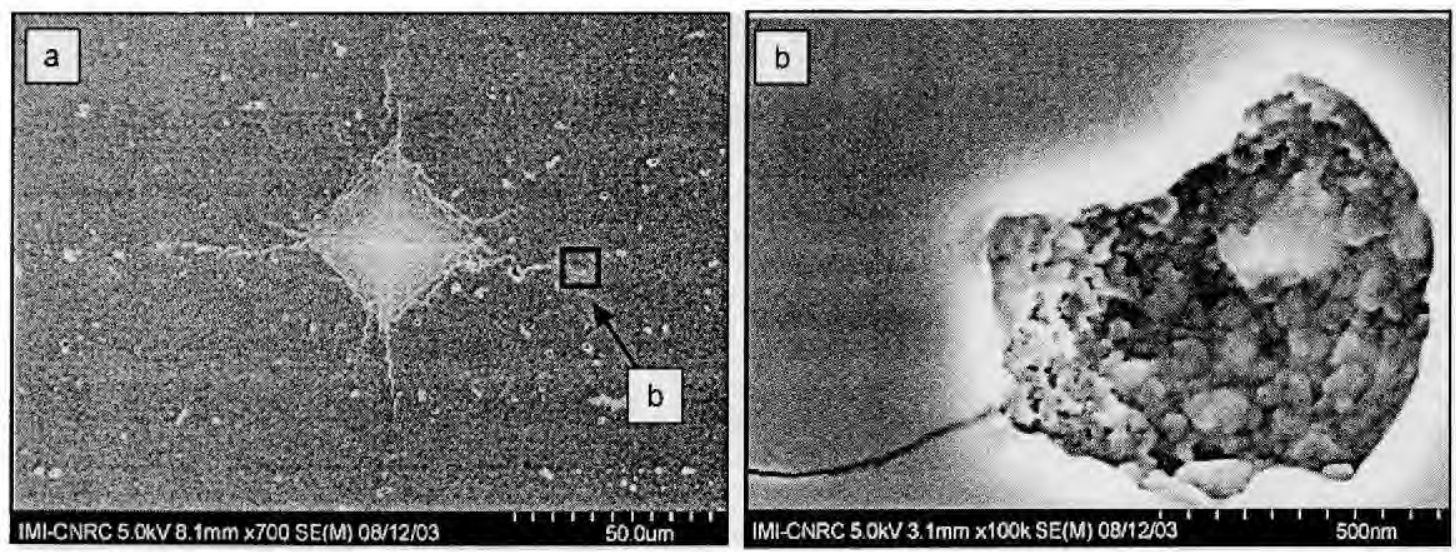

Fig. 2.7: Vickers indentation impression (1 kgf) in the cross-section of the HVOF-

sprayed nanostructured $\mathrm{TiO}_{2}$ coating (a) and the indentation crack tip being arrested by a zone of nanostructured particles (b) [6]. 


\subsection{Nature and Properties of Aluminum Oxide}

$\mathrm{Al}_{2} \mathrm{O}_{3}$ is white and odorless oxide ceramic (also known as alumina or aluminum oxide), with a hexagonal crystal structure shown in Fig. 2.8. In nature, $\mathrm{Al}_{2} \mathrm{O}_{3}$ is the hardest mineral after diamond with a hardness of 18-20 GPa. Due to its high hardness and refractory nature, $\mathrm{Al}_{2} \mathrm{O}_{3}$ is widely used as thermal liners, thermal barrier installations, high temperature insulating systems, crucibles, ceramic boards and brackets, heaters, etc. Crystal structure, physical, thermal, and mechanical properties of $\mathrm{Al}_{2} \mathrm{O}_{3}$ are presented in Table 2.3.

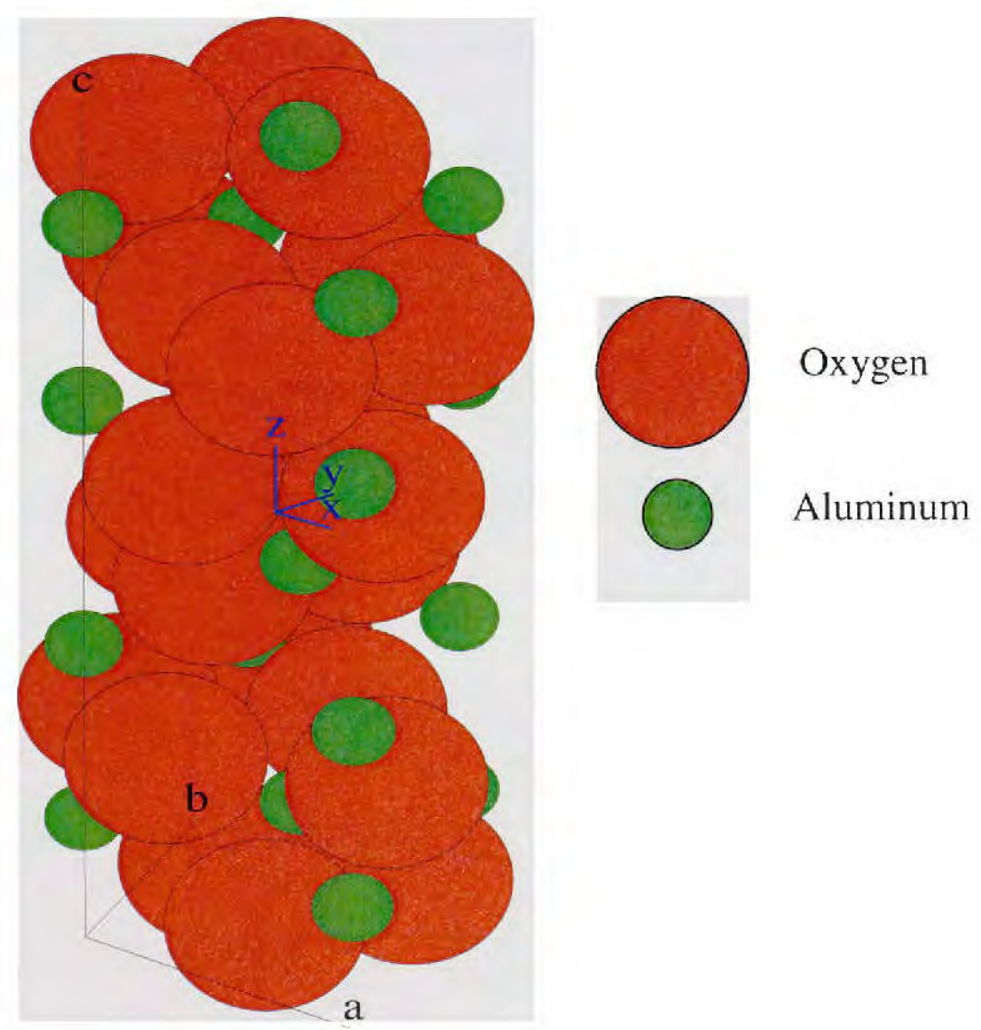

Fig. 2.8: Crystal Structure of $\mathrm{Al}_{2} \mathrm{O}_{3}\left(\mathrm{a}=\mathrm{b}=4.7564 \AA, \mathrm{c}=12.9894 \AA\right.$, and $\alpha=\beta=90^{\circ}, \gamma$ $\left.=120^{\circ}\right)$. 
Table 2.3: Crystal Structure, physical, thermal and mechanical properties of $\mathrm{Al}_{2} \mathrm{O}_{3}$

\begin{tabular}{|c|c|c|}
\hline Properties of $\mathrm{Al}_{2} \mathrm{O}_{3}$ & Description & Details \\
\hline 1. Crystal Structure & Hexagonal & $\begin{array}{l}\text { lattice parameters } \mathrm{a}=\mathrm{b}= \\
4.7564 \AA, \mathrm{c}=12.9894 \AA \\
\text { and } \alpha=\beta=90^{\circ}, \gamma=120^{\circ}\end{array}$ \\
\hline 2. Physical Properties & $\begin{array}{l}\text { Melting Point } \\
\text { Density } \\
\text { Molar Volume } \\
\text { Molecular Weight }\end{array}$ & $\begin{array}{l}2327 \mathrm{~K} \\
3.99 \mathrm{~kg} / \mathrm{m}^{3} \\
25.554 \mathrm{~cm}^{3} / \mathrm{mol} \\
101.96 \mathrm{~g} / \mathrm{mol}\end{array}$ \\
\hline 3. Thermal Properties & $\begin{array}{l}\text { Coeff. Of Therm. Expansion } \\
\text { Specific Heat } \\
\text { Thermal Conductivity }\end{array}$ & $\begin{array}{l}6.76 \times 10^{-6} \mathrm{~K}^{-1} \\
78.7 \mathrm{~J} / \mathrm{mol} / \mathrm{K} \\
36.16 \mathrm{~W} / \mathrm{m} / \mathrm{K}\end{array}$ \\
\hline 4. Mechanical Properties & $\begin{array}{l}\text { Young's Modulus } \\
\text { Poisson's Ratio } \\
\text { Hardness } \\
\text { Fracture Toughness } \\
\text { - Monolithic } \\
\text { - Plasma Sprayed }\end{array}$ & $\begin{array}{l}389.5 \mathrm{GPa} \\
0.22 \\
18-20 \mathrm{GPa} \\
\\
\sim 3.2 \mathrm{MPa} \mathrm{m}^{1 / 2} \\
\sim 2.0 \mathrm{MPa} \mathrm{m}^{1 / 2}\end{array}$ \\
\hline
\end{tabular}

Aluminum oxide has several polymorphs. Among many polymorphs of aluminum

oxide, $\alpha-\mathrm{Al}_{2} \mathrm{O}_{3}$ (with space group $\mathrm{R} \overline{3} \mathrm{c}$ ) is the most thermodynamically stable form. Other metastable phases can be classified into: (i) face-centered closed (fcc) packing as: cubic $(\gamma, \eta)$, monoclinic $(\theta)$, tetragonal/orthorhombic $(\delta)$, (ii) hexagonal closed packing (hcp) as rhombohedral $(\alpha)$, orthorhombic $(\kappa)$, and hexagonal $(\chi)$. Other monoclinic phases are identified as $\theta^{\prime}, \theta^{\prime \prime}$, and $\lambda[14]$.

Though atomic stacking for oxygen ion is similar in amorphous and $\gamma-\mathrm{Al}_{2} \mathrm{O}_{3}$, transformation from $\gamma$ - to $\alpha-\mathrm{Al}_{2} \mathrm{O}_{3}$ is primarily associated to rearrangement of oxygen sublattice as shown in Fig. 2.9. Difference in the activation energy of amorphous $\rightarrow \gamma$ transition $(4.5 \mathrm{eV})$ compared to the activation energy of $\gamma \rightarrow \alpha$ transformation $(5.2 \mathrm{eV})$ suggests that the mechanism of atomic rearrangements controlling these 
a)

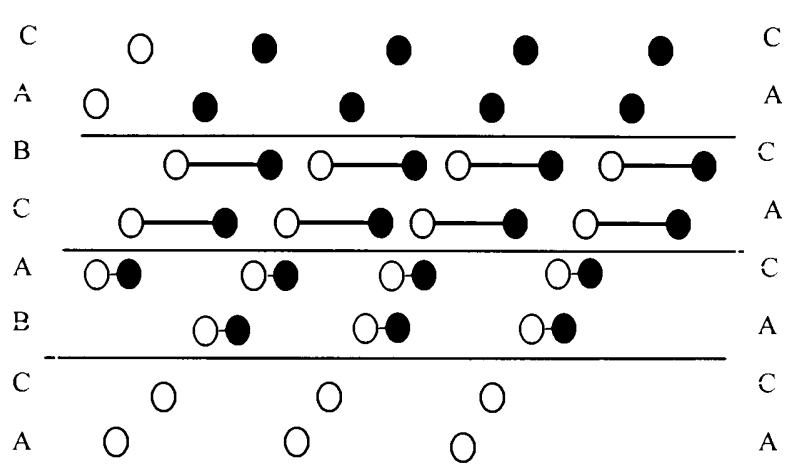
$\bigcirc$ f.c.c
$[11 \overline{2}]$
h.c.p

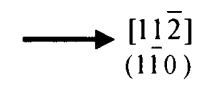

b)

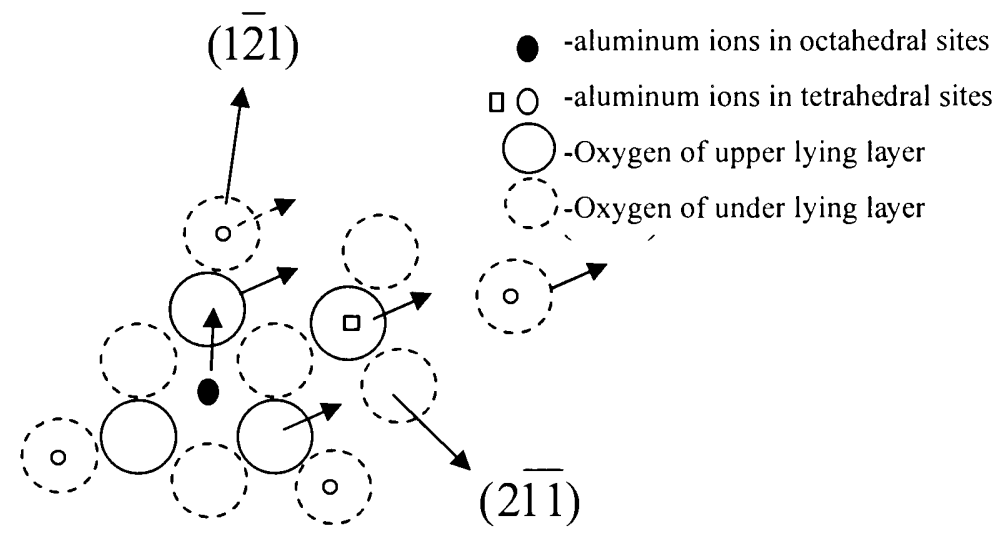

c)

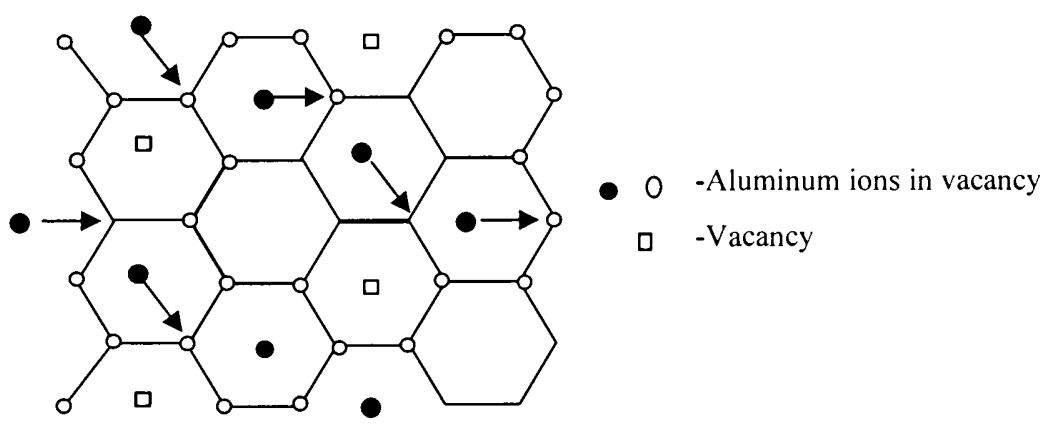

Fig. 2.9: (a) Change of stacking of $\{111\}$ spinel oxygen layers from fcc to hcp. (b) Shear of an oxygen layer having aluminum cations in the interstices. (c) Cooperative migration of the cations around regularly distributed vacancies, resulting in a "honey comb" lattice for $\alpha-\mathrm{Al}_{2} \mathrm{O}_{3}[14]$. 
transformations is not the same [14]. No experimental confirmations are available till date to clearly explain direct $\gamma \rightarrow \alpha$ transformation mechanism. But following orientation relationships are presented between $\gamma$ and $\alpha \mathrm{Al}_{2} \mathrm{O}_{3}:<001>_{\gamma} / /<0001>_{\alpha},\{440\}_{\gamma} / /\{30 \overline{3} 0\}_{\alpha}$, and $\{310\}_{\gamma} / /\{\overline{2} 110\}_{\alpha}$ determined from electron diffraction [14].

Transformation concepts are important in understanding the grain growth and phase evolution of $\mathrm{Al}_{2} \mathrm{O}_{3}$ during processing. These aspects become highly important especially in rapid processing techniques that can form metastable phases, such as thermal spraying, rapid melt quenching, vapor deposition, laser processing, etc [72-74]. Correspondingly, understanding of solid-state transformations associated with $\mathrm{Al}_{2} \mathrm{O}_{3}$ processing become beneficial in attaining required properties via optimizing the microstructure.

\subsection{Ceramics Processing via Thermal Spraying}

Thermal spraying was practiced since early 1900s where flame was utilized as heat source for melting the material [41]. Since the development of plasma spray torch by Thermal Dynamic Corp. in 1957, plasma spraying has established itself in depositing thick ceramic coatings $(>50 \mu \mathrm{m})$. High velocity oxy-fuel (HVOF) spraying, vacuum plasma spraying (VPS), detonation-gun (D-gun) spraying and cold spraying are other processes in the family of thermal spray HVOF utilizes high velocity of carrier gas (3-5 
mach) and uses combustion as source of thermal energy. Deposition of coatings in HVOF and D-gun is achieved through plastic deformation with secondary assistance from thermal energy. Hence microstructure is usually observed with minimal porosity (and high density) in HVOF and D-gun sprayed coatings. Deposition of coating in cold spray is achieved primarily through plastic deformation. Hence, cold spray process is limited to deposition of soft metallic coatings. Plasma spraying utilizes thermal energy as the primary source to melt the powders and deposit consolidated coatings. Thermal spraying has evolved as effective processing tool to synthesize ceramic coatings with improved properties such as fracture toughness, indentation crack resistance, spallation resistance against bend and cup test, adhesion strength, abrasive wear resistance and sliding wear resistance [75-84]. Table 2.4 shows a list of ceramics processed by thermal spray processes. Conventional thermal spray processes such as wire arc spraying and flame oxy-fuel spraying, etc are not considered here.

Since plasma spraying of $\mathrm{Al}_{2} \mathrm{O}_{3}$ based coatings is the focus of current research, HVOF, VPS and D-gun processing of ceramics is not discussed here. Owing to ease of attaining high temperatures in plasma plume (excess of $10000 \mathrm{~K}$ ) and being able to melt any known material, processing of ceramics has made plasma spraying the first choice [101]. Its ease of use and low cost associated with spraying has helped its wide commercialization. Plasma spraying has emerged as material processing techniques in 
spite of its inception of specifically depositing coatings [41]. Plasma spraying involves several processing parameters such as plasma power, primary and secondary gases types and their flow rates, powder feedstock (size, shape and morphology), feed rate of powder

Table 2.4: Fabrication of ceramics by thermal spraying

\begin{tabular}{|c|c|c|}
\hline Ceramics & Thermal Spraying Processing & References \\
\hline Non-Oxides & VPS & {$[85]$} \\
$\mathrm{MoSi}_{2}-\mathrm{Si}_{3} \mathrm{~N}_{4}$ & HVOF & {$[86]$} \\
$\mathrm{TiC}-\mathrm{Ni}$ & VPS & {$[87]$} \\
$\mathrm{TaC}$ & Controlled APS & {$[88]$} \\
$\mathrm{SiC} / \mathrm{ZrB}_{2}$ & APS & {$[89]$} \\
$\mathrm{WC}$ & & {$[90]$} \\
\hline Oxides & APS & {$[91]$} \\
$\mathrm{Al}_{2} \mathrm{O}_{3}$ & HVOF, APS & {$[92]$} \\
$\mathrm{Al}_{2} \mathrm{O}_{3} / \mathrm{SiC}$ & APS & {$[93]$} \\
${\mathrm{Mo}-\mathrm{MoO}_{2}}_{\mathrm{ZrO}_{2}-\mathrm{Al}_{2} \mathrm{O}_{3}}$ & APS & {$[94]$} \\
$\mathrm{Al}_{2} \mathrm{O}_{3}-\mathrm{TiO}_{2}$ & APS & {$[6,95-97]$} \\
$\mathrm{TiO}_{2}$ & HVOF, VPS & {$[98]$} \\
$\mathrm{ZrO}_{2}$ & APS & {$[12]$} \\
$\mathrm{Al}_{2} \mathrm{O}_{3}-\mathrm{Ni}$ & HVOF & {$[99]$} \\
$\mathrm{HAP}_{-}-\mathrm{ZrO}$ & HVOF & {$[54]$} \\
$\mathrm{HAP}_{2}-\mathrm{CNT}^{\mathrm{HAP}^{*}}$ & APS & {$[100]$} \\
\hline
\end{tabular}

VPS: Vacuum Plasma Spraying, APS: Air/Atmospheric Plasma Spraying, HVOF: High Velocity Oxy Fuel, D-Gun: Detonation-Gun HAP*: Hydroxyapatite 
(and its flowability), external cooling, coating/formed shape thickness and many more $[41,101]$. In addition secondary parameters such as thermal conductivity of powder particles, application of external cooling, power fluctuations, inconsistent flow of powders due to gravity, etc also add to the complexity of process. Following section discusses plasma spraying in detail.

\subsubsection{Plasma Spraying}

Plasma is the fourth state of matter, namely ionized gas. An arc is struck between tungsten cathode and copper anode to generate plasma, where the temperature reaches in excess of $10,000 \mathrm{~K}$. Plasma spraying involves feeding powder particles in the plasma stream with pressurized gases creating velocities upto Mach 1-3 [102-104]. A schematic of plasma spraying process is presented in Fig. 2.10. Powders experience differential heat (temperature) and velocity owing to their location, size and morphology. Molten/semimolten particles exiting from the plasma plume get resolidified and impact on the substrate to form a coating. Successive deposition lead to increasing thickness of the layer, which can be subsequently obtained as a free standing structure in case the substrate is separated from the deposit [87]. 


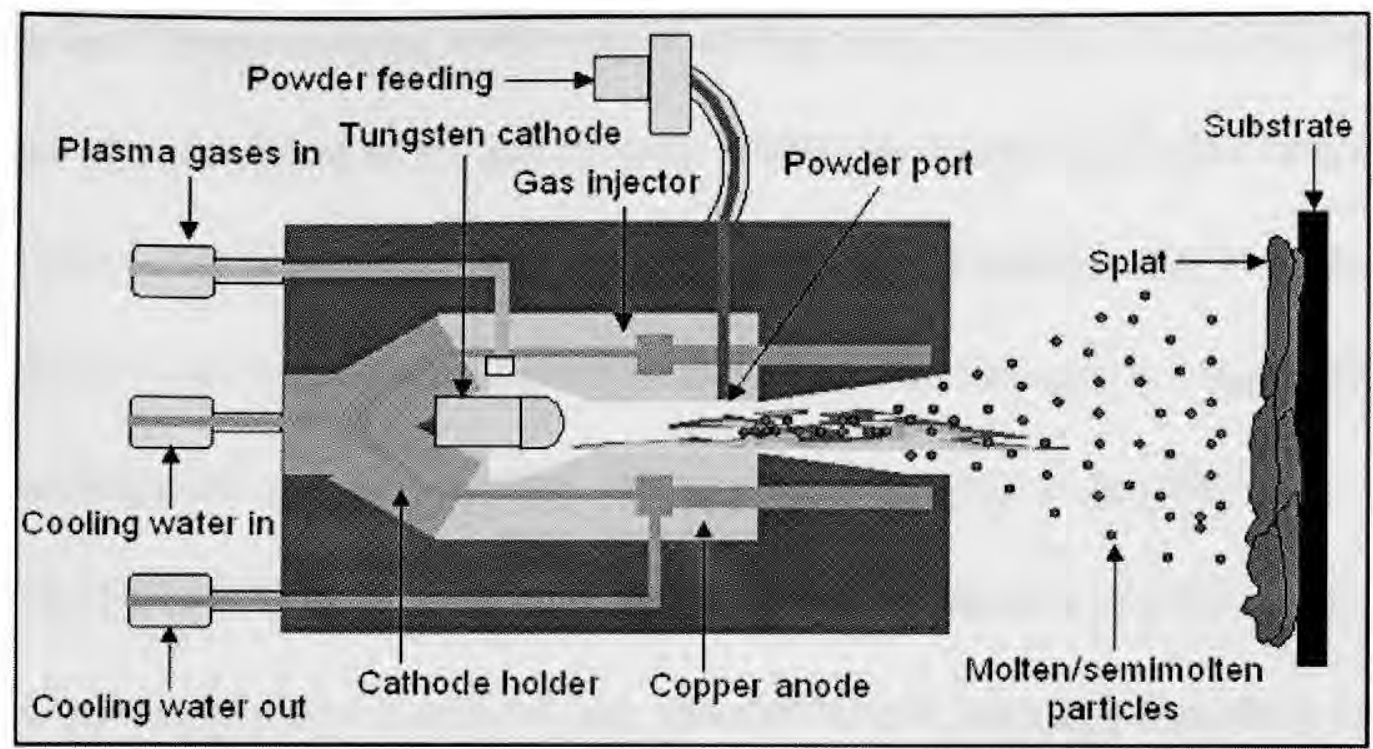

Fig. 2.10: Schematic of plasma spraying process.

\subsubsection{Plasma Sprayed Nanocrystalline Ceramic Coatings}

Plasma spraying has been utilized by several researchers to synthesize nanocrystalline ceramic coatings $[33,73,75]$. With stringent material requirements, ceramic nanocomposites (such as WC-Co, Mo-Si-B, Hydroxyapatite, $\mathrm{FeAl}, \mathrm{Y}_{2} \mathrm{O}_{3}-\mathrm{ZrO} 2$, $\mathrm{ZrO}_{2}-\mathrm{Al}_{2} \mathrm{O}_{3}, \mathrm{Al}_{2} \mathrm{O}_{3}-\mathrm{TiO}_{2}$, etc) have been plasma sprayed for improved friction, wear, biocompatibility, oxidation resistance etc. $[36,73,81,90,105-110]$. Nanostructured coatings also provide a solution to improving the fracture toughness of the ceramics. Grain size refinement provides the required strength and improved toughness due to HallPetch relationship [51, 52].

Synthesis of nanocrystalline ceramic coating by plasma spraying is associated with two major challenges: (i) grain growth and (ii) lack of flowability of nanosize 
powders. Plasma spraying involves rapid cooling rates $\left(\sim 10^{6} \mathrm{~K} / \mathrm{s}\right)$ and formation of fine grain size is inherent to the process [43]. Additional cooling can further prevent grain growth during plasma spraying. However, low mass of nanoparticles and subsequent inability to be carried in gas stream for deposition onto substrate is a major challenge. Flowability of such fine powders during plasma spraying is limited owing to high surface/volume ratio. In order to reduce interparticle friction and enhance flowability, nanoparticles must be constituted into spherical micron sized agglomerates via powder treatment. Thereby, spray drying as necessary powder pretreatment in atomizing nanoparticles as spherical micron sized agglomerates becomes a requirement. Also, the limitation of flowing nanoparticles can be obviated by passing powders as liquid powder feed. Liquid evaporates and leaves the nanoparticles as coating on the substrate. Reduced deposition rates associated with solution precursor plasma spraying makes the process less attractive. Current research has adopted spray drying for achieving good flowability of nanoparticles, which is explained in the following section.

\subsubsection{Spray Drying of Ceramic Nanopowders}

Spray drying is a process utilizing spraying of fluid powder-feed (powder dispersed in solvent- (generally alcohol) or water-based suspensions), and consequent drying (with nitrogen or air) to result spherical agglomerated mass of dry powder [111]. 
Organic binder is added to keep the solid particles combined with one another. Characteristics of the spray-dried powder are influenced by: type of atomizer, composition and viscosity of suspension, feed rate, drying temperature and atomizing pressure. Spray-dried powders possess high flowability and thereby its coherent flow results high densities and precise thickness in the plasma sprayed structures $[112,113]$.

Spray drying is a complex process involving (i) droplet formation, (ii) evaporation and ballooning, (iii) droplet explosion, and (iv) particle formation, Fig. 2.11 [111]. When suspension is pumped into the atomizing chamber, it fragments into small droplets by compressed feed gas. Consequent evaporation of solvent causes shrinkage of the droplet in the second stage. During the process, liquid takes along solid particles from core to the outside of the droplet creating a void when evaporation rate inside the droplet exceeds the diffusion rate through the droplet. When pressure exceeds a certain limit, thickness of droplet is not sufficient to hold the ballooning by expansion of gases in its core, causing explosion in some cases. Final stage is recognized by shrinkage from surface tension until it completely dries. Spray dried powders are spherical and free flowing, which are consequently sieved to the ideal powder particle size range of $15-60 \mu \mathrm{m}$ required for plasma spraying. High quality thermal spraying of micro and nanostructured coatings such as WC-Co, TaC, and $\mathrm{Al}_{2} \mathrm{O}_{3}-\mathrm{TiO}_{2}$ are described in literature $[33,87,98,114]$. 
Plasma spraying of spray-dried powders may reduce interlamellar boundaries/cracks and may give increased thermal corrosion and wear resistance [115-117].

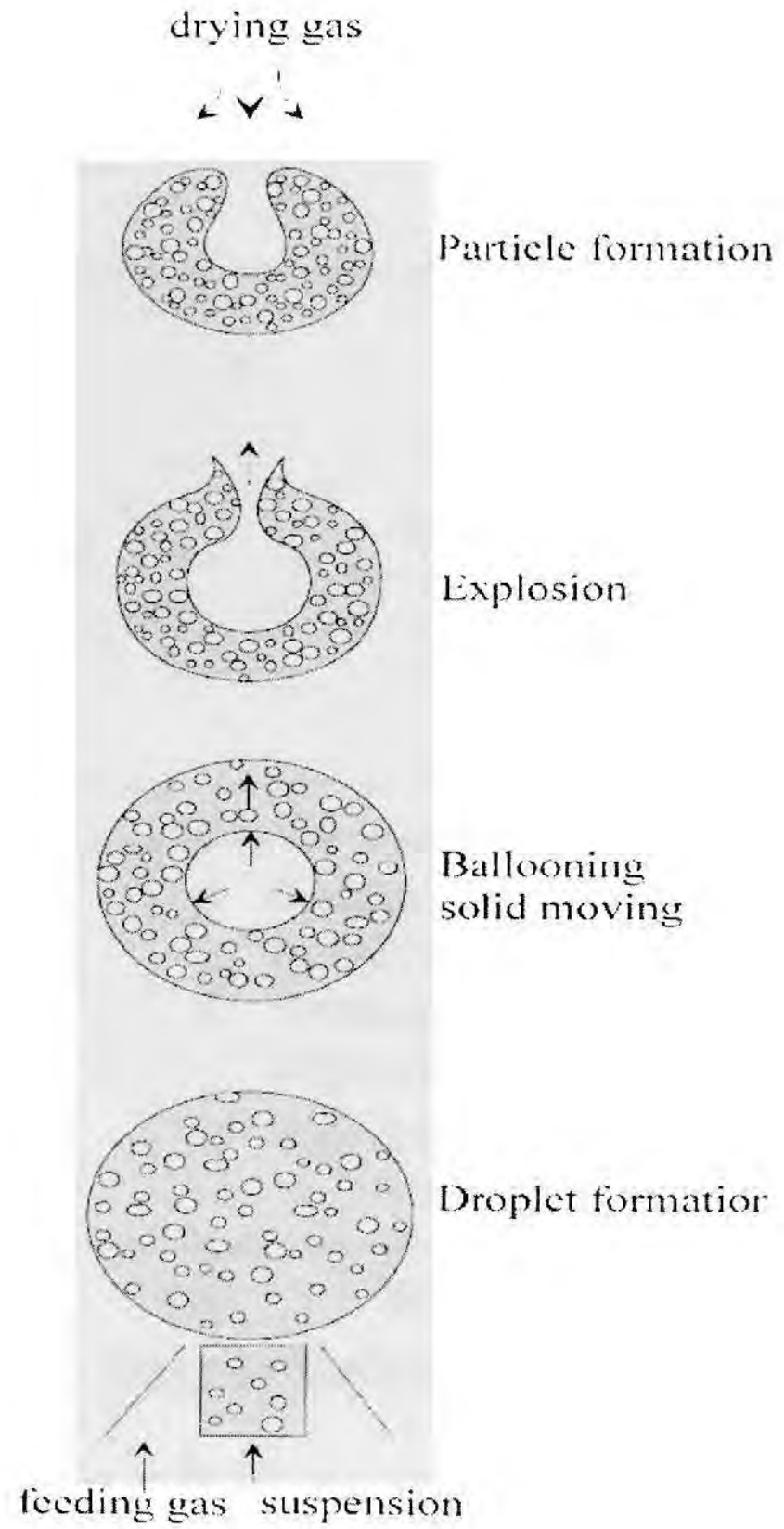

Fig. 2.11: Spray drying of ceramics [111]. 
Plasma sprayed coatings nano- $\mathrm{ZrO}_{2}$ exhibited excellent dynamic modulus $(\sim 170$ GPa) and enhanced damping capacity as observed in Fig. 2.12 [13]. TEM micrograph of nano $\mathrm{ZrO}_{2}$ coating in Fig. 2.13 revealed fine grains in the order of $70-100 \mathrm{~nm}$. Improved damping capacity was attributed to enhanced grain-boundary relaxations.

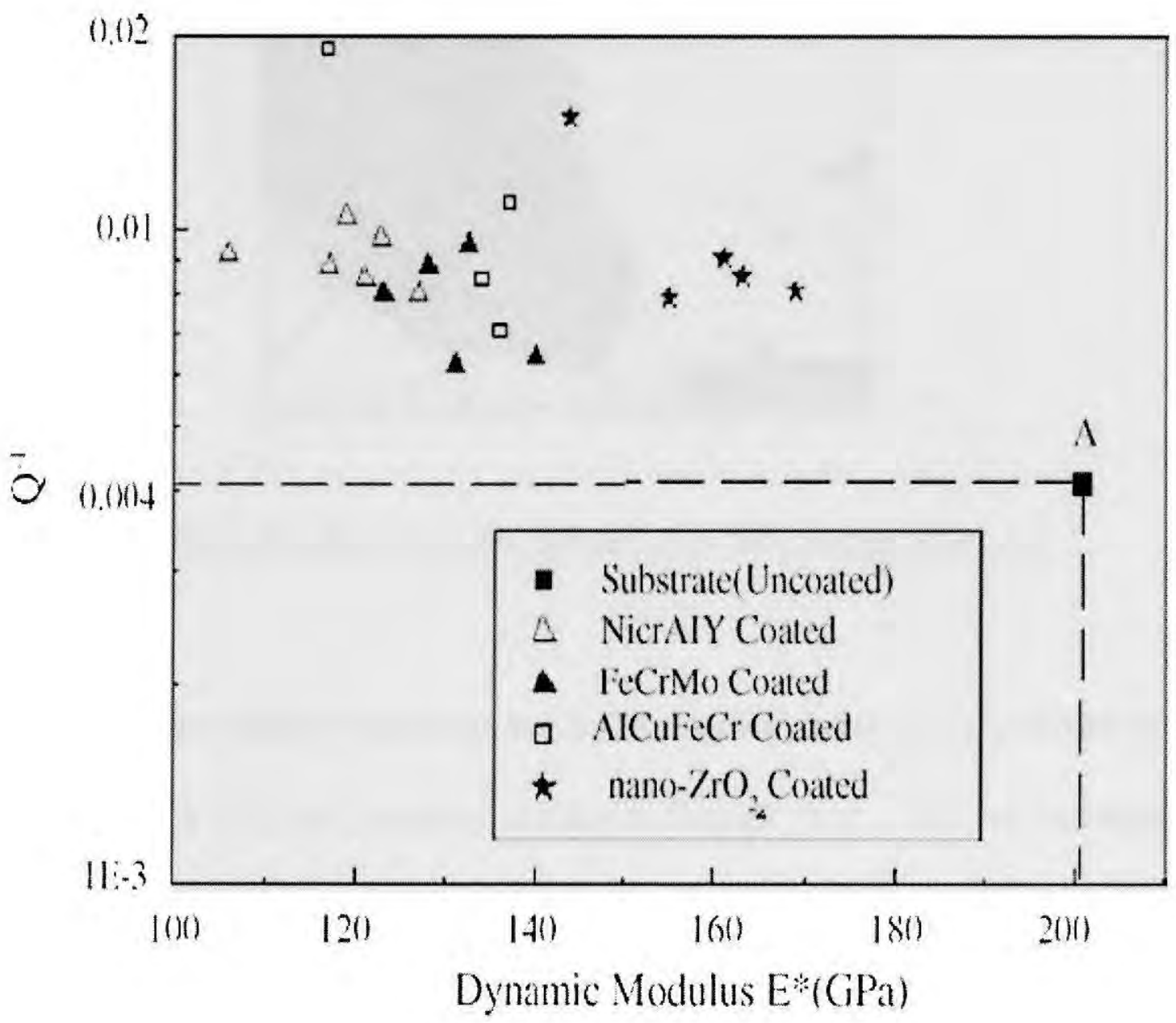

Fig. 2.12: Damping capacity vs dynamic modulus map of various thermally sprayed samples [13]. 


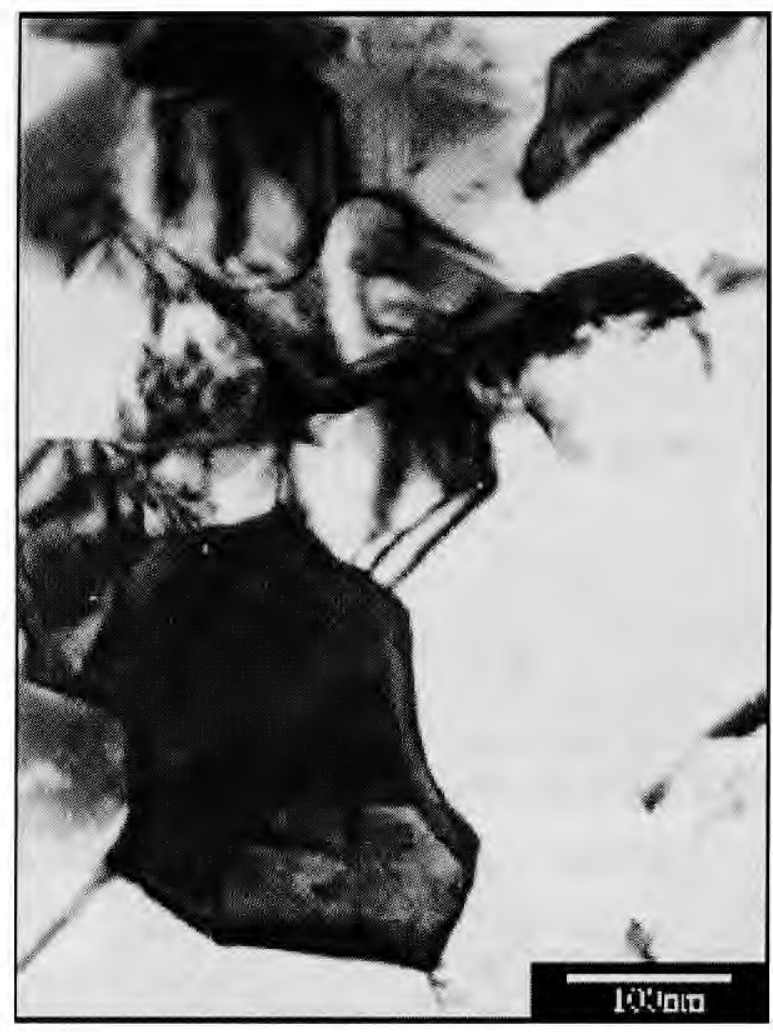

Fig. 2.13: TEM micrograph of plasma sprayed nano- $\mathrm{ZrO}_{2}$ coating layer [13].

Another study by Goberman and Dell focused on plasma spraying of nano $\mathrm{Al}_{2} \mathrm{O}_{3}$ $13 \mathrm{wt} \% \mathrm{TiO}_{2}[73,94]$. Secondary electron micrograph (Fig. 2.14a) and backscattered electron micrograph (Fig. 2.14b) elicited bimodal microstructure in nano $\mathrm{Al}_{2} \mathrm{O}_{3}-13$ wt $\%$ $\mathrm{TiO}_{2}$ coatings. Presence of bimodal microstructure assisted toughening of the coatings. In order to define the processing parameter, a critical plasma spray parameter (CPSP) was defined, as equation 2.2, for the plasma spraying with keeping powder feed rate, gun speed, carrier gas flow rate, spray distance and flow ratio of Ar to $\mathrm{H}_{2}$ as constant [73]: 

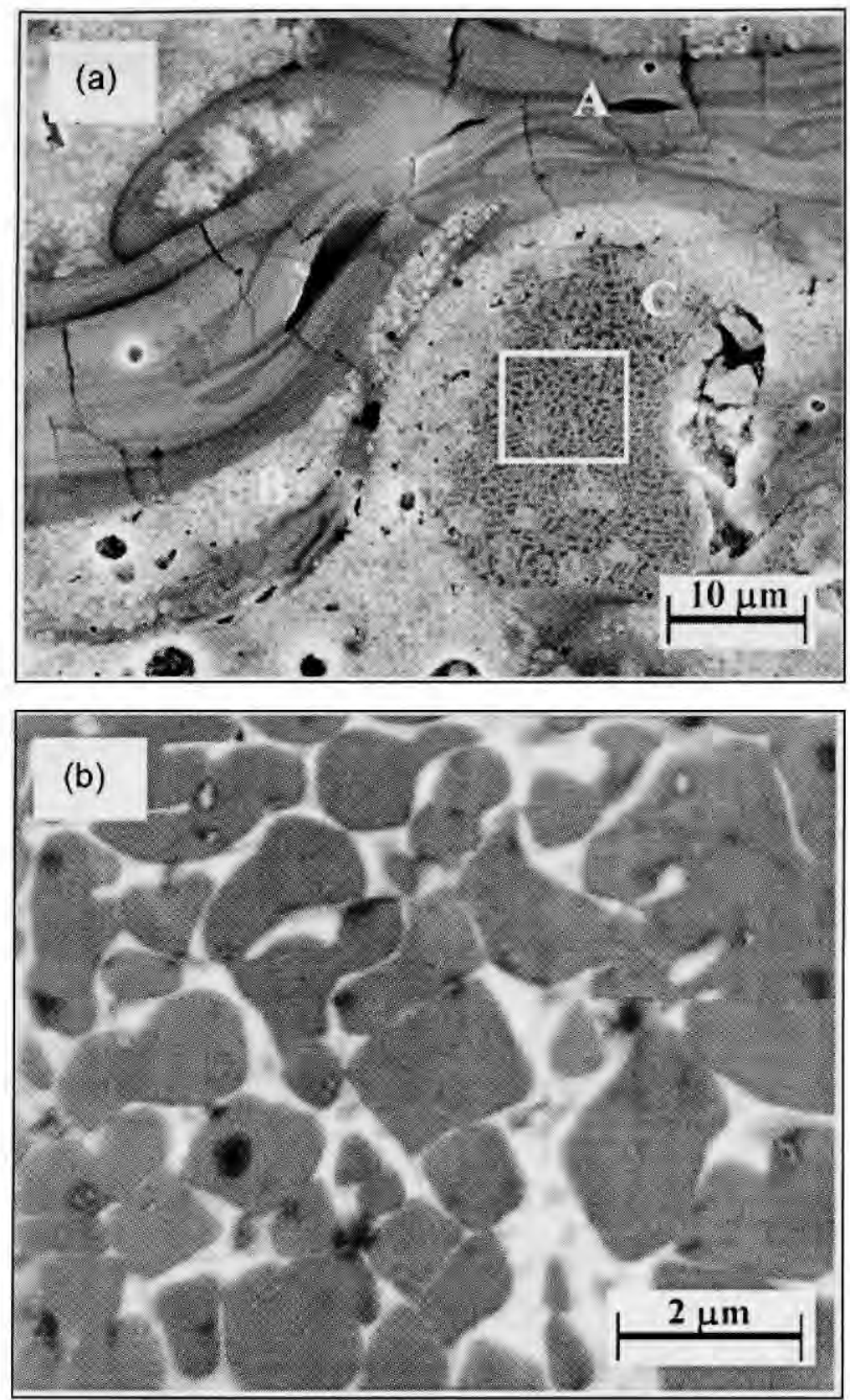

Fig. 2.14: a) Secondary and b) Backscattered electron micrographs of plasma sprayed nano- $\mathrm{Al}_{2} \mathrm{O}_{3}-13$ wt. $\% \mathrm{TiO}_{2}$ with additives. (a) Regions of splats (region $\mathrm{A}$ ) and small particulates (region C), and (b) The backscattered electron image illustrates that the embedded particles appear darker than in the matrix [94]. 


$$
\mathrm{CPSP}=\text { voltage } x \text { current / primary gas }(\text { Ar) flow rate Equation } 2.2
$$

CPSP was related directly to plasma torch/particle temperature. It was evident that metastable phases were inherent in thermal spray processes owing to non-equilibrium nature of fabrication technique $[73,94,118-120]$. Generation of metastable nanocrystalline $\gamma-\mathrm{Al}_{2} \mathrm{O}_{3}$ phase was observed to vary with varying CPSP, Fig. 2.15 [94].

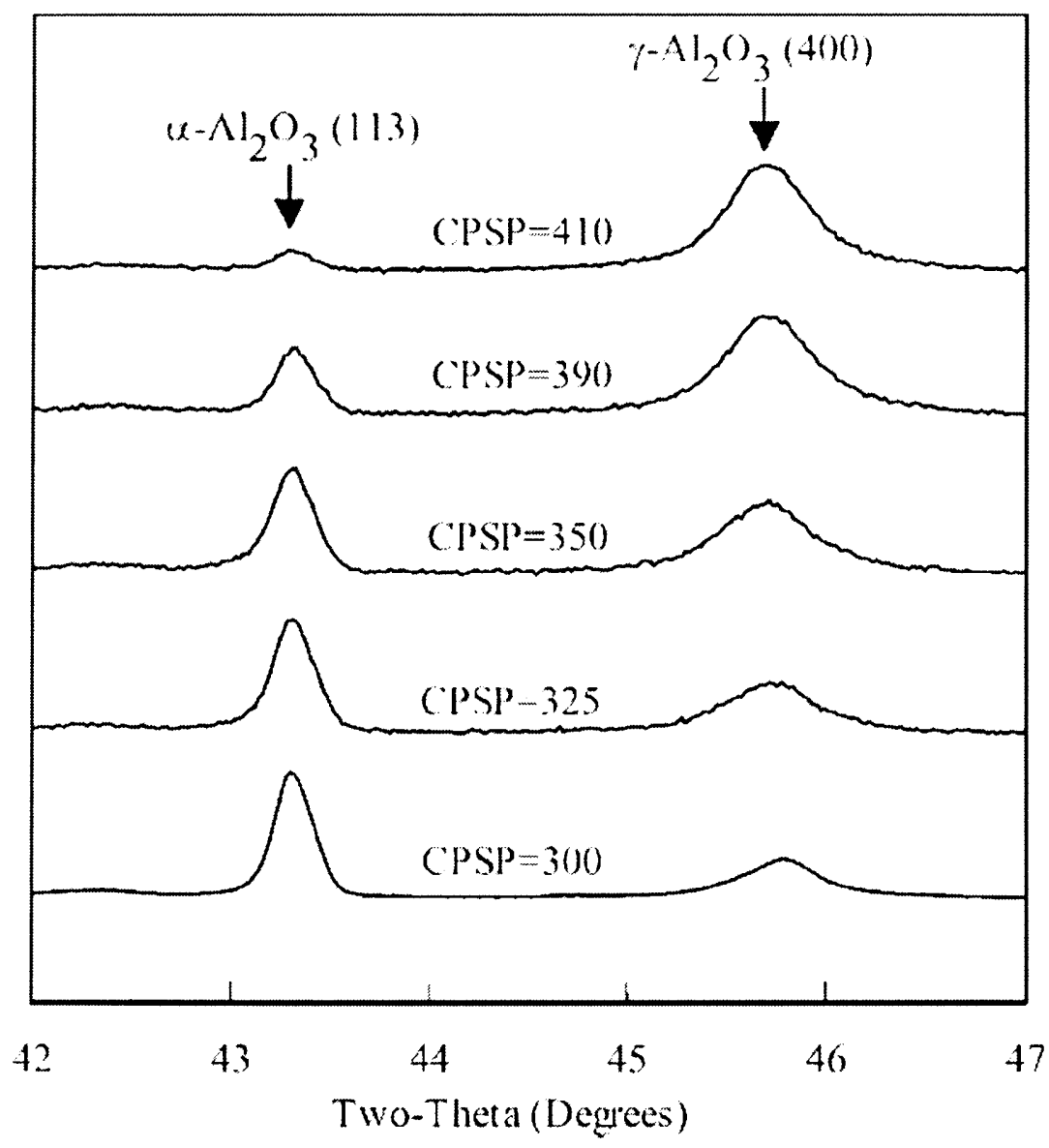

Fig. 2.15: X-ray diffraction patterns from (113) $\alpha-\mathrm{Al}_{2} \mathrm{O}_{3}$ and (400) $\gamma-\mathrm{Al}_{2} \mathrm{O}_{3}$ peaks for reconstituted $\mathrm{Al}_{2} \mathrm{O}_{3}-13 \mathrm{wt} . \% \mathrm{TiO}_{2}$ with (modified) additives. The relative integrated intensity of these peaks are observed to vary as a function of CPSP [94]. 


\subsection{Toughening of Aluminum Oxide}

Toughening of $\mathrm{Al}_{2} \mathrm{O}_{3}$ is worked upon by using secondary phases as additives, consolidating nanocrystalline $\mathrm{Al}_{2} \mathrm{O}_{3}$ powder particles via pressing and sintering, and reinforcing with particulates, fibers or whiskers [35, 121-136]. Following example elicit specific examples of toughened $\mathrm{Al}_{2} \mathrm{O}_{3}$ ceramic.

Ultrafine grained $(1-50 \mathrm{~nm})$ bulk $\mathrm{Al}_{2} \mathrm{O}_{3}$ ceramic was pressed at $1120 \mathrm{MPa}$ and sintered at $1150^{\circ} \mathrm{C}$ to evince enhanced resistance to mechanical and thermal shock [121]. Moreover, metastable phases of $\mathrm{Al}_{2} \mathrm{O}_{3}$ such as gamma and theta nucleate as secondary precipitates and assist in precipitation strengthening of $\mathrm{Al}_{2} \mathrm{O}_{3}$ ceramic[122-124]. $\mathrm{Li}$ et al demonstrated that nano $\alpha-\mathrm{Al}_{2} \mathrm{O}_{3}$ can be obtained using two-step sintering, Fig. 2.16 [121]. Along with sintering additives, sintering time and temperature have a strong effect on the generated microstructure making sintering an exciting technique to alter the microstructure and attain enhanced structural properties in nanoceramics [125, 126]. Since fracture toughness of $\mathrm{Al}_{2} \mathrm{O}_{3}$ is lower than stress required to initiate plastic deformation, role of additives in enhancing the energy absorption (by generating banded structure, Fig. 2.17) becomes more prominent $[127,128]$. Table 2.5 lists role of various additives in enhancing the fracture toughness of $\mathrm{Al}_{2} \mathrm{O}_{3}$ ceramic composites. Though introduction of fibers, particulates and whiskers was highly initiated, the recent trend is using CNTs as an effective reinforcement, and is discussed in detail in the next section. 


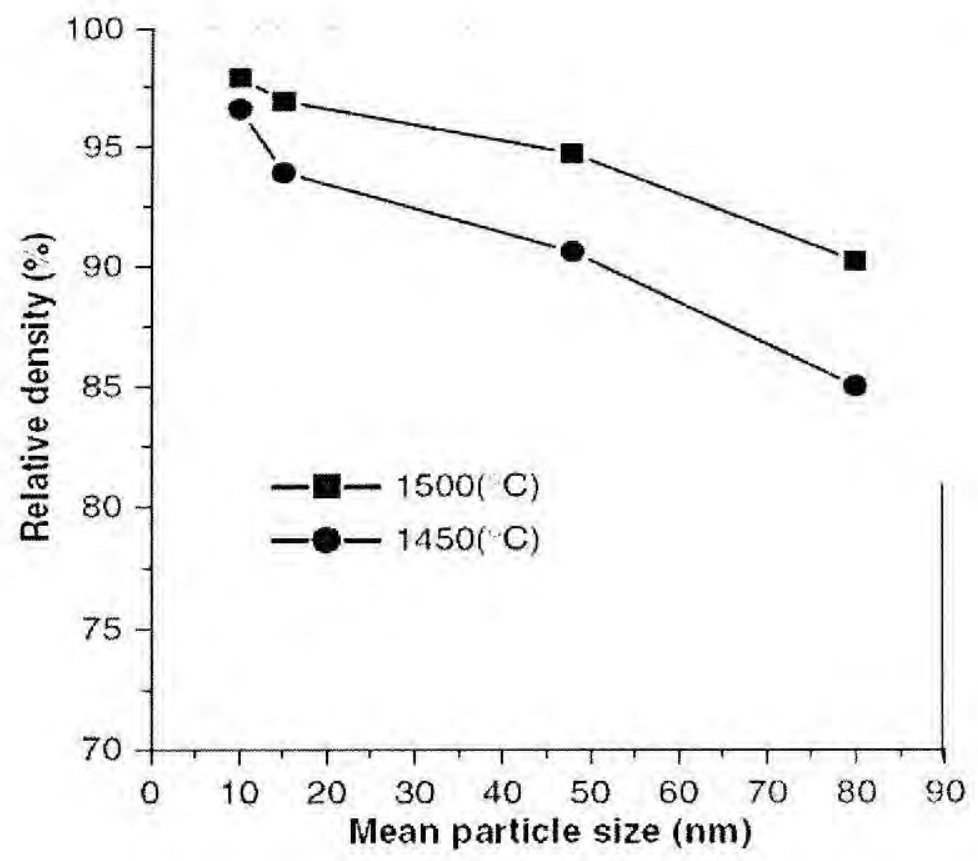

Fig. 2.16: The relative density of the $\mathrm{Al}_{2} \mathrm{O}_{3}$ nanoceramics pressed at $1120 \mathrm{MPa}$ and sintered at different temperatures for $5 \mathrm{~h}$ as a function of mean particle size of the starting $\alpha-\mathrm{Al}_{2} \mathrm{O}_{3}$ nanopowders [121].

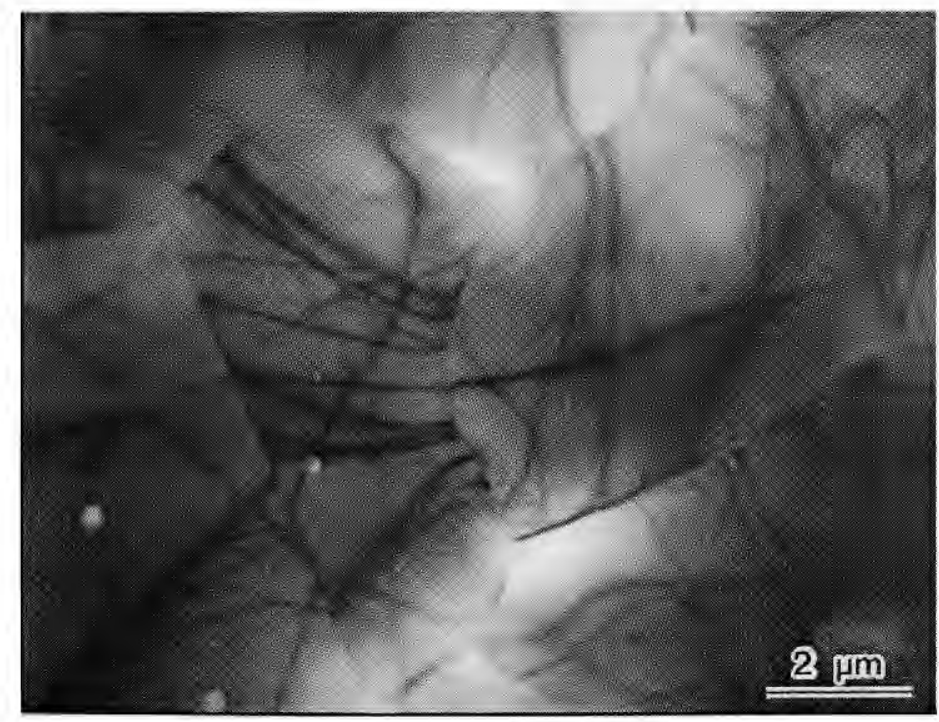

Fig. 2.17: TEM micrograph of an $\mathrm{Al}_{2} \mathrm{O}_{3}-1 \mathrm{~mol} \% \mathrm{TiO}_{2}-1 \mathrm{~mol} \% \mathrm{MgO}$ solid solution sintered at $1500^{\circ} \mathrm{C}$ for $5 \mathrm{~h}$ in air [127]. 
Table 2.5: Role of additives in enhancing fracture toughness of $\mathrm{Al}_{2} \mathrm{O}_{3}$

\begin{tabular}{|c|c|c|c|c|}
\hline Ceramic/Metal & Additive (P/F/W) & Fracture Toughness & Toughening Mechanism & Reference \\
\hline $\mathrm{Al}_{2} \mathrm{O}_{3}$ & $\begin{array}{c}\text { Yttria Stabilized } \\
\text { Zirconia }(\mathrm{P})\end{array}$ & $8.9 \mathrm{MPa} \cdot \mathrm{m}^{1 / 2}$ & $\begin{array}{c}\text { Ferroelastic domain switching and } \\
\text { transformation toughening }\end{array}$ & {$[125]$} \\
\hline $\mathrm{Al}_{2} \mathrm{O}_{3}$ & $\mathrm{SiC}(\mathrm{W})$ & $3.8 \mathrm{MPa} \cdot \mathrm{m}^{1 / 2}$ & Crack healing by SiC whisker & {$[35]$} \\
\hline $\mathrm{MgAl}_{2} \mathrm{O}_{4}$ & $\mathrm{Al}_{2} \mathrm{O}_{3}(\mathrm{P})$ & $2.5-5.82 \mathrm{MPa} \cdot \mathrm{m}^{1 / 2}$ & $\begin{array}{c}\text { Grain boundary sliding and creep } \\
\text { diffusion }\end{array}$ & {$[129]$} \\
\hline $\mathrm{Al}_{2} \mathrm{O}_{3}$ & $\begin{array}{c}\text { Yttrium Aluminum } \\
\text { Garnet }(\mathrm{P})\end{array}$ & $4.54 \mathrm{MPa} \cdot \mathrm{m}^{1 / 2}$ & $\begin{array}{c}\text { YAG inclusion leading to residual } \\
\text { stress. Reduction of grain and flaw } \\
\text { size. }\end{array}$ & {$[34]$} \\
\hline $\mathrm{Al}_{2} \mathrm{O}_{3}$ & $\mathrm{MgSiO}_{3}$ & $3.3 \mathrm{MPa} \cdot \mathrm{m}^{1 / 2}$ & $\begin{array}{c}\text { Stepped and micro-faceted } \\
\text { cleavage }\end{array}$ & {$[130]$} \\
\hline $\mathrm{Al}_{2} \mathrm{O}_{3}$ & $\begin{array}{c}\text { Monoclinic } \mathrm{Zirconia} \\
(\mathrm{P})\end{array}$ & $11.8 \mathrm{MPa} \cdot \mathrm{m}^{1 / 2}$ & Phase transformation toughening & {$[131]$} \\
\hline $\mathrm{Al}_{2} \mathrm{O}_{3}$ & $\mathrm{CeO}_{2}(\mathrm{P})$ & $4.7 \mathrm{MPa} \cdot \mathrm{m}^{1 / 2}$ & Particulate toughening & {$[132]$} \\
\hline $\mathrm{Ni}^{\mathrm{Al}} \mathrm{O}_{3}(\mathrm{~F})$ & $10-35 \mathrm{MPa} \cdot \mathrm{m}^{1 / 2}$ & Metallic Bridging layer & {$[133]$} \\
\hline $\mathrm{Al}, \mathrm{Al}-\mathrm{Cu}$ & $\mathrm{Al}_{2} \mathrm{O}_{3}(\mathrm{P})$ & $6.5-30 \mathrm{MPa} \cdot \mathrm{m}^{1 / 2}$ & $\begin{array}{c}\text { High stress triaxiality between } \\
\text { narrowly spaced ceramic particles, } \\
\text { ductile matrix, and high Young's } \\
\text { modulus }\end{array}$ & {$[134,135]$} \\
\hline $\mathrm{Al}$ & $\mathrm{Al}_{2} \mathrm{O}_{3}(\mathrm{~F})$ & $\sim 4.8 \mathrm{MPa} \cdot \mathrm{m}^{1 / 2}$ & $\begin{array}{c}\text { Plastic deformation of the metal, } \\
\text { testing at 1200 }{ }^{0} \mathrm{C}\end{array}$ & {$[136]$} \\
\hline
\end{tabular}

* P: Powder, F: Fiber, W: Whisker 


\subsection{Carbon Nanotubes as Reinforcement for Nanocomposite for Improving Mechanical Properties}

Nanocomposite is a novel class of materials that combines two or more materials, physically and/or chemically distinct, in creating a resultant material in which size of one of the phases is less than 100 nanometer [137]. The advantage of using nanocomposites is achieving the best qualities of the constituted material and often some, which are even superior to those of constituent materials possess.

Carbon nanotube (CNT) is a new class of material that has superior mechanical properties with theoretical tensile strength of $200 \mathrm{GPa}$ and Young's modulus reaching $\sim 1$ TPa $[11,22,25,37,38]$. CNTs have also displayed superplasticity despite their $\mathrm{sp}^{2}$ bonding [138]. Superplastic deformation of upto $280 \%$ in Fig. 2.18, hints CNT as an ideal reinforcement for toughening ceramics. Such high deformation is attributed to kink nucleation and movement apart from atomic movement to heal vacancies [138]. These properties of CNTs make them suitable candidate for as reinforcement for creating nanocomposites with improved mechanical properties.

Wavelike distortions were observed in repeated bending of the CNTs with local strains of upto $16 \%$ without failure, Fig. 2.19 [139]. The bending modulus of CNTs decreased with the increasing diameter of CNTs. Sustained integrity at large strain with no sign of brittleness, plasticity or atomic arrangement inferred good resilience [140]. Hence CNTs serve as potential reinforcements for improving ductility in the ceramic nanocomposites. In addition, excellent mechanical properties of CNTs do not restrict their participation in enhancing the strength of polymer-CNT and metal-CNT nanocomposites as discussed in the consequent sections. 


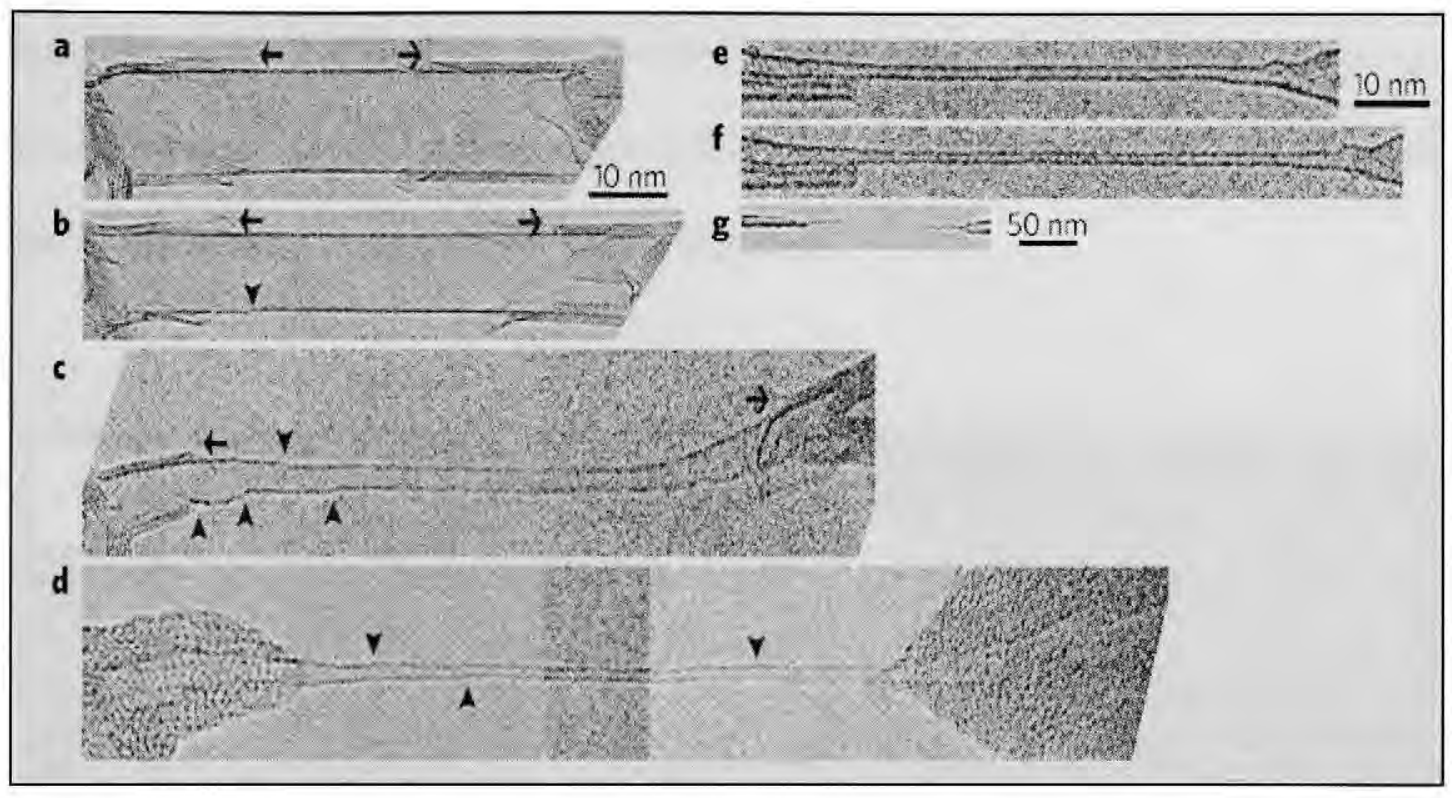

Fig. 2.18: Insitu tensile elongation of individual single walled carbon nanotubes viewed under HRTEM. Tensile elongation under a-d) constant bias of $2.3 \mathrm{~V}$, and e-g) without bias. Vertical arrows show the kinks and horizontal arrows represent the kink movement direction in carbon nanotubes [138].

\subsubsection{CNT Reinforced Polymer Matrix Nanocomposites}

Most of the research on CNT reinforced nanocomposites has been carried out on polymer matrix. CNT reinforcement in matrices such as UHMWPE (ultra high molecular weight polyethylene), PMMA (polymethylmethacrylate), PS (polystyrene), PAN (polyacrylonitrile), PMEMA (Methyl-ethyl methacrylate copolymer), etc have been utilized [38, 141-146]. The main purpose of adding CNTs in polymer matrix had been to improve the elastic modulus and tensile strength via effective load transfer between polymer and CNT. Table 2.6 summarizes CNT reinforced polymer nanocomposites and 
improvement in mechanical properties [142-146]. CNT dispersion, polymer-CNT bonding, and CNT pullout are dictated as the main reasons of strengthening the nanocomposite. Chemical interaction at the CNT-polymer interface are critical and affect the toughening of polymer-CNT nanocomposite.

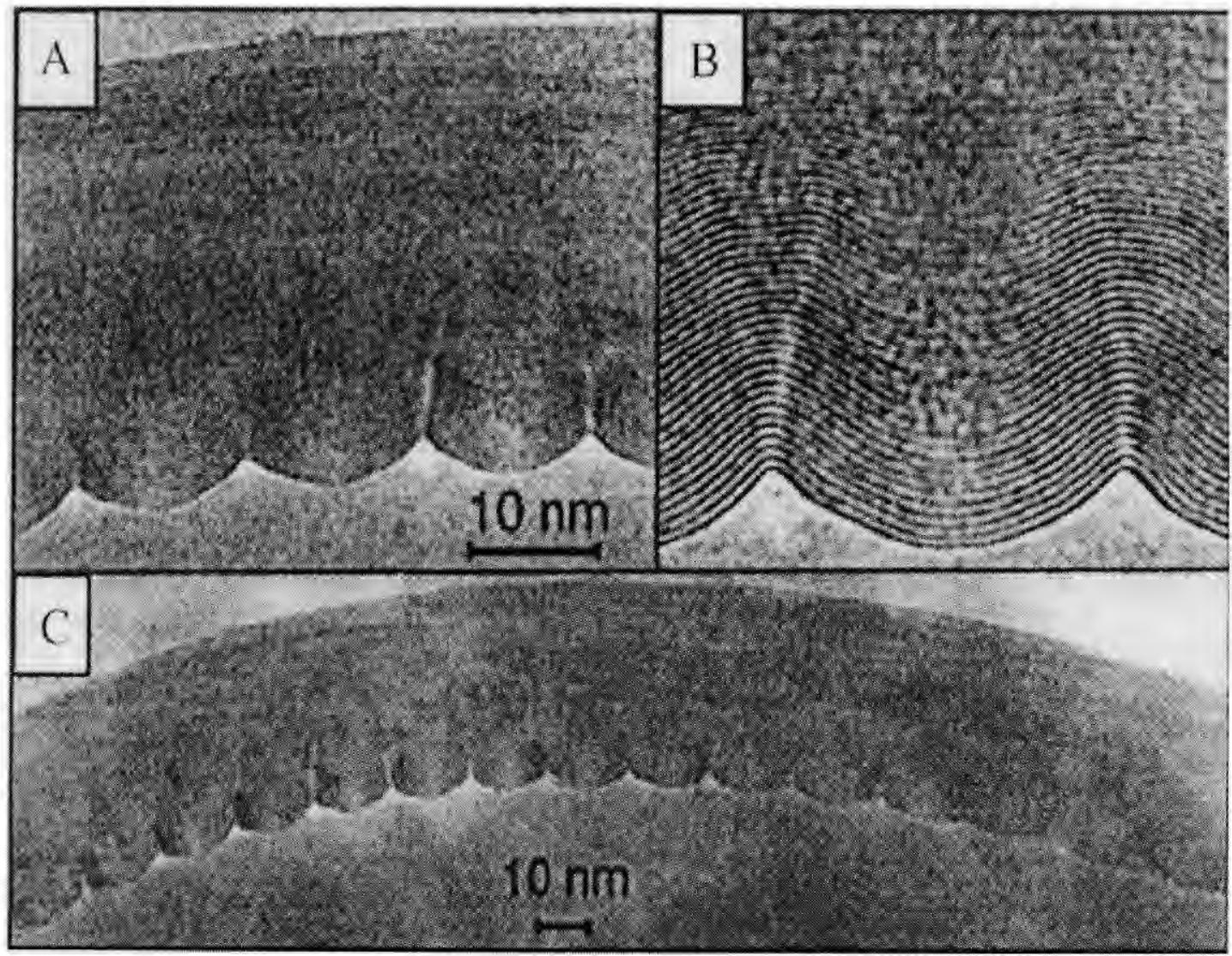

Fig. 2.19: TEM image of bent $\mathrm{CNT}$ with $\mathrm{A}$ and $\mathrm{B}$ ) radius of curvature $\sim 40 \mathrm{~nm}$, and $\mathrm{C}$ ) amplitude of ripples increase at the outer layer [140].

Polymer-CNT reinforcement has shown enhanced role of CNT in load transfer, as seen in Fig. 2.20 via CNT pulling and bridging [141]. Modulus and strength improvements of 
Table 2.6: CNT-reinforced polymer nanocomposites

\begin{tabular}{|c|c|c|c|c|}
\hline Polymer & $\begin{array}{c}\text { CNT } \\
\text { Loading }\end{array}$ & $\begin{array}{c}\text { Microstructural } \\
\text { Features }\end{array}$ & Mechanical Property & Reference \\
\hline Epoxy & 0.5 wt. $\%$ & $\begin{array}{l}\text { CNT pullout. } \\
\text { Intimate CNT } \\
\text { contact with } \\
\text { matrix. Ductile } \\
\text { deformation in } \\
\text { fractured surface. }\end{array}$ & $\begin{array}{c}\text { Increase in Young's } \\
\text { modulus by } 200 \% \\
\text { (upto } 10.9 \mathrm{MPa} \text { ). } \\
\text { Tensile strength } \\
\text { increase by } 140 \% \\
\text { (upto } 0.44 \mathrm{GPa} \text { ) (softer } \\
\text { epoxy matrix) }\end{array}$ & [142] \\
\hline Polypropylene & 1 wt. $\%$ & $\begin{array}{l}\text { Well dispersed } \\
\text { CNTs. Good } \\
\text { wetting observed. }\end{array}$ & $\begin{array}{c}\text { Enhancement of } \\
\text { Young's Modulus } \\
\text { (from } 5.2 \text { to } 8.0 \mathrm{GPa} \text { ) } \\
\text { and tensile strength } \\
\text { increased from } 7.9 \text { to } \\
9.8 \mathrm{GPa} .\end{array}$ & {$[143]$} \\
\hline $\begin{array}{l}\text { PMMA (poly } \\
\text { methyl } \\
\text { methacrylate) }\end{array}$ & 4 wt. $\%$ & $\begin{array}{l}\text { Lack of chemical } \\
\text { interaction } \\
\text { between PMMA } \\
\text { and CNT. Weaker } \\
\text { interfacial } \\
\text { adhesion resulted } \\
\text { higher toughness. }\end{array}$ & $\begin{array}{l}\text { No improvement in } \\
\text { Young's Modulus. } \\
\text { Considerable } \\
\text { improvement in } \\
\text { impact resistance } \\
\text { (from } 40 \text { to } 160 \\
\mathrm{KJ} / \mathrm{m}^{2} \text { ) }\end{array}$ & {$[144]$} \\
\hline Epoxy & 5 wt. $\%$ & $\begin{array}{l}\text { CNT aggregation } \\
\text { limited reaching } \\
\text { theoretical } \\
\text { predicted values. } \\
\text { CNT bundles } \\
\text { easily slide pass } \\
\text { one another. }\end{array}$ & $\begin{array}{c}\text { Elastic Modulus } \\
\text { increase by } 75 \%(\sim 7 \\
\text { GPa }) \text { and hardness } \\
\text { increase by } 30 \% \\
(\sim 0.42 \mathrm{GPa})\end{array}$ & {$[145]$} \\
\hline Polystyrene & 1 wt. $\%$ & $\begin{array}{c}\text { Good CNT } \\
\text { dispersion. Good } \\
\text { interfacial bonding }\end{array}$ & $\begin{array}{l}\sim 42 \% \text { increase in } \\
\text { elastic modulus (from } \\
1.19 \text { to } 1.69 \mathrm{GPa} \text { ) and } \\
\sim 25 \% \text { increase in } \\
\text { break stress (from } \\
12.8 \text { to } 16 \mathrm{MPa} \text { ) }\end{array}$ & {$[146]$} \\
\hline
\end{tabular}


upto $200 \%$ and toughness improvements of upto $150 \%$ have been reported in epoxyCNT nanocomposites [141]. Since matrix provides a secondary role in bearing the stress, stress transfer to the reinforcing CNT fiber is highly critical [147, 148]. Importance of CNT incorporation as a dispersed phase is impressed upon by various research works, with dispersion being achieved by sol-gel technique, surface activation, inorganic coating, heterocoagulation, ball milling, solvent incorporation, etc [28, 149-155].

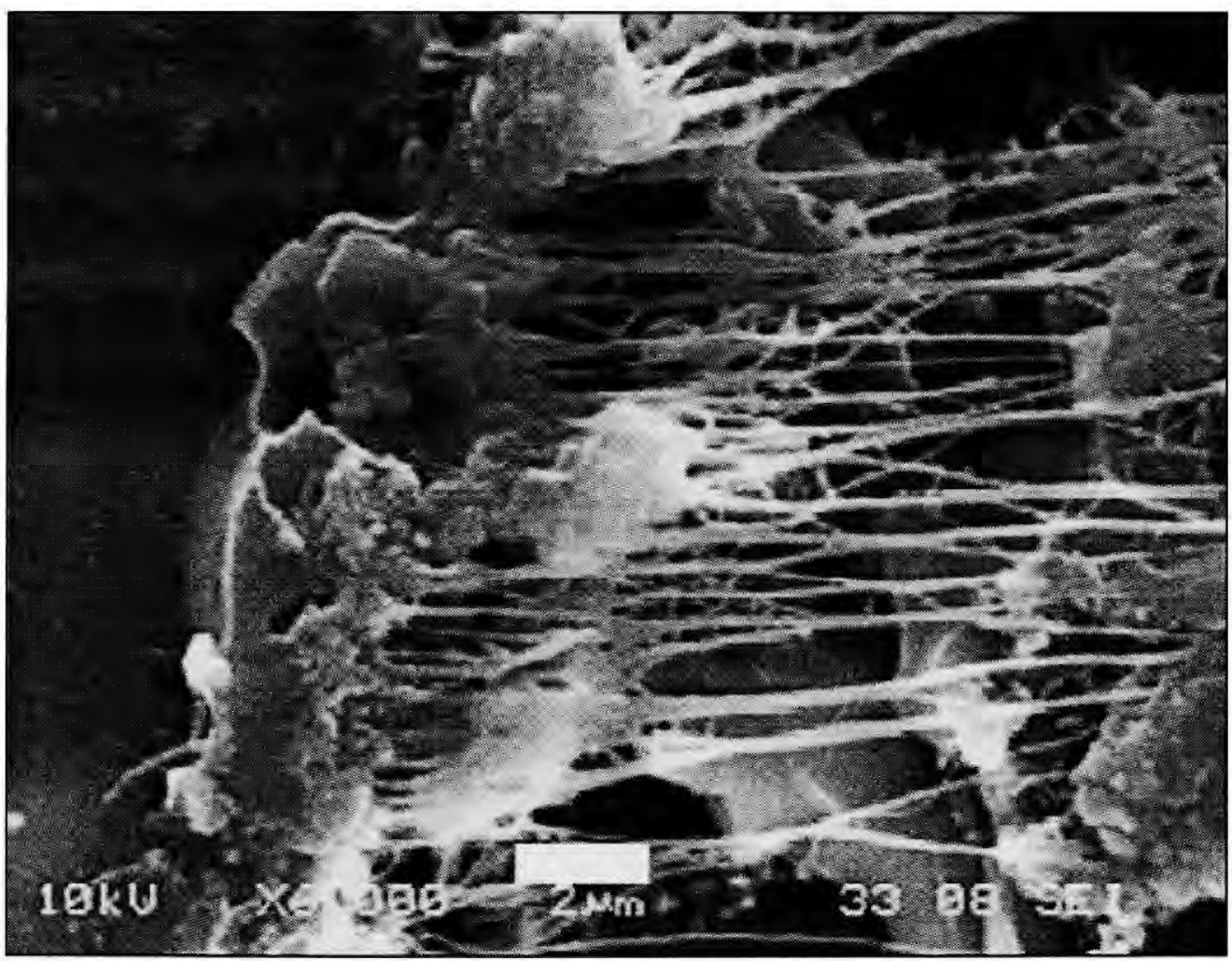

Fig. 2.20: CNT ropes are observed bridging a fatigue fracture surface in an epoxy matrix [141],

Though literature has continually reported enhancement of tensile strength and modulus experimentally, these values are far below the theoretical predictions. The difference between experimental observations and theoretical predictions has been 
attributed to poor dispersion of CNT in the matrix and lack of understanding in engineering CNT/matrix interface [38, 141, 144]. Next class of nanocomposite is CNT reinforced metal-matrix composite as explained in section 2.5.2.

\subsubsection{CNT Reinforced Metal Matrix Nanocomposites}

Comparatively limited research has been performed on metal matrix-CNT nanocomposites [156-158]. Basic challenge arising in the metal-CNT nanocomposite is associated with damage to CNTs at high temperature processing, as required for metal processing $[43,44,118]$. Secondary challenge includes low interfacial bonding strength between CNT and metal interface [156-158].

CNT addition in $\mathrm{Al}$ and Ti matrix has shown improvement in elastic modulus and hardness [43]. Spark plasma sintered and isostatically pressed Cu-CNT nanocomposites depicted enhanced wear resistance upto three times whereas hardness increased two times $[159,160]$. A summary of improvement in mechanical properties of metal-CNT nanocomposites is presented in Table 2.7.

CNT dispersion in the metal matrix is critical to impart stress transfer towards improving strength, and elastic modulus [164]. Dangling CNTs in the fractured surface of the plasma sprayed Al-Si/CNT nanocomposite is presented in Fig. 2.21a [44] indicating strong anchoring by CNTs. Smooth CNT surface indicated no physical damage during plasma spraying. Survival of CNTs was possible because of rapid kinetics inherent to the plasma spray processing. Laha et la has investigated $\mathrm{CNT} /$ metal interface in $\mathrm{Al}-\mathrm{Si} / \mathrm{CNT}$ system $[43,44,118]$. It was concluded that a thin layer of SiC on CNT is formed (Fig. 2.21 b) suggesting interfacial reaction which improved interface wettability required for 
Table 2.7: Mechanical property enhancement in metal-CNT nanocomposite

\begin{tabular}{|c|c|c|c|c|}
\hline Metal & $\begin{array}{c}\text { CNT } \\
\text { content }\end{array}$ & Microstructure & Property & Reference \\
\hline $\mathrm{Cu}$ & 1 vol. $\%$ & $\begin{array}{l}\text { Severe plastic deformation } \\
\text { (Equi channel angular } \\
\text { pressing). Homogeneous } \\
\text { deformation }\end{array}$ & $\begin{array}{l}\text { Increase in indentation } \\
\text { load by upto } 20 \% \\
\text { (from } 56 \text { to } 75 \mathrm{kgf} \text { for } \\
300 \mu \mathrm{m} \text { deformation. }\end{array}$ & [156] \\
\hline $\mathrm{Sn}-\mathrm{Ag}-\mathrm{Cu}$ & $\begin{array}{c}0.07 \\
\text { wt. } \%\end{array}$ & $\begin{array}{l}\text { Extruded. Addition of } \\
\text { CNTs enhanced the } \\
\text { wetting behavior. Large } \\
\text { difference in CTE } \\
\text { induced multidirection } \\
\text { thermal stress. }\end{array}$ & $\begin{array}{l}\text { Improvement in yield } \\
\text { strength from } 31 \text { to } 36 \\
\mathrm{MPa} \text {, microhardness } \\
\text { increase from } 16.3 \text { to } \\
17.0 \mathrm{Hv}\end{array}$ & {$[157]$} \\
\hline $\mathrm{Ti}$ & $\begin{array}{c}20 \text { vol. } \\
\%\end{array}$ & $\begin{array}{l}\text { Hot pressed. Dispersion } \\
\text { strengthening, refinement } \\
\text { of grain size, and TiC } \\
\text { formation }\end{array}$ & $\begin{array}{c}\text { Hardness increase } \\
\text { from } 221 \text { to } 1216 \mathrm{H}_{\mathrm{V}} \\
\text { and Young's Modulus } \\
\text { increase from } 120 \text { to } \\
198 \mathrm{GPa} \text {. }\end{array}$ & [158] \\
\hline $\mathrm{Mg}$ & 2 wt. $\%$ & $\begin{array}{l}\text { Hot pressed. Uniform } \\
\mathrm{CNT} \text { dispersion in Mg } \\
\text { matrix. Improved } \\
\text { interface bonding and } \\
\text { effective load transfer. } \\
\text { Upto } 98 \% \text { dense. }\end{array}$ & $\begin{array}{c}\text { Young's modulus } \\
\text { improvement by } 9 \% \\
\text { (from } 35.3 \text { to } 38.6 \\
\mathrm{GPa} \text { ) }\end{array}$ & [161] \\
\hline $\mathrm{Ag}$ & 8 vol. $\%$ & $\begin{array}{l}\text { Pressing and sintering. } \\
\text { Uniform distribution of } \\
\text { CNTs. Agglomeration } \\
\text { observed for higher CNT } \\
\text { content }(>10 \%) \text {. CNT } \\
\text { pullout, CNT bridging, } \\
\text { and CNT alignment. }\end{array}$ & $\begin{array}{c}9 \% \text { increase in bend } \\
\text { strength (from } 427 \text { to } \\
465 \mathrm{MPa} \text { ) }\end{array}$ & [162] \\
\hline $\mathrm{Al}-\mathrm{Si}$ & 10 wt. $\%$ & $\begin{array}{l}\text { CNT retention after } \\
\text { plasma spraying. SiC } \\
\text { layer over CNT surface }\end{array}$ & $\begin{array}{c}\text { Elastic modulus } \\
\text { increased from } 79 \text { to } \\
114.5 \mathrm{GPa} \text {. UTS of } \sim \\
234 \mathrm{MPa}\end{array}$ & $\begin{array}{c}{[43,44} \\
163]\end{array}$ \\
\hline
\end{tabular}


effective load transfer between metal/CNT interface. CNT reinforced ceramic nanocomposites are discussed in the next section.

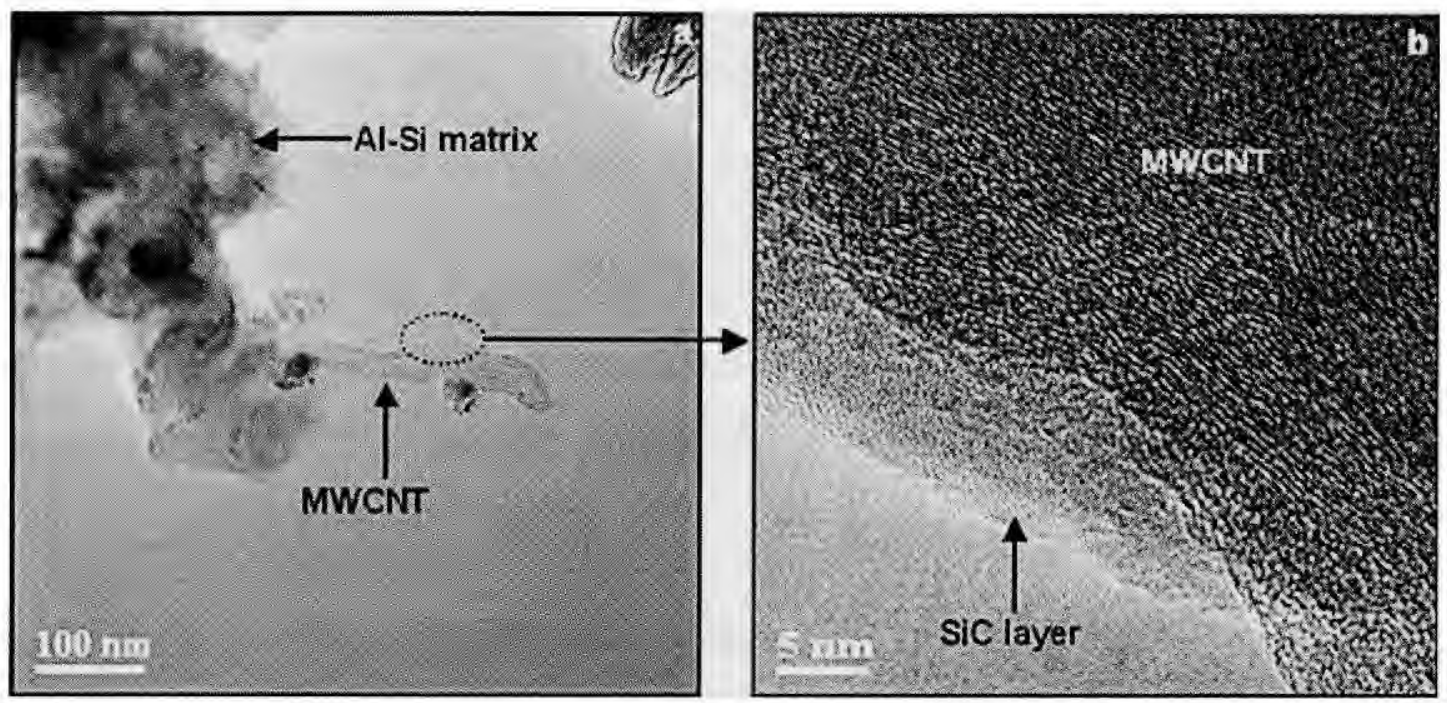

Fig. 2.21: Retention and CNT pullout is observed in the high magnification SEM image of fractured surface of Al-CNT composite [44].

\subsubsection{CNT Reinforced Ceramic Nanocomposites:}

Incorporation of fibers and CNTs in the ceramic matrix has shown promising results in improving the fracture toughness of ceramic matrix. $[9,17,21-31]$. Toughening behavior of CNT reinforced matrix is mainly linked to CNT crack bridging, CNT dispersion and crack deflection $[17,165,166]$. It has been theoretically dictated that spherical particles do not contribute to toughness of ceramic composites [18]. Hence research directed to using non-spherical particles/fibers/nanotubes in enhancing the fracture toughness of ceramic nanocomposites is well justified. Temporal evolution of CNT reinforced ceramics by various processing techniques is presented in Table 2.8 . 
Hot pressing of $\mathrm{Fe}-\mathrm{Al}_{2} \mathrm{O}_{3}$-CNT composite depicted continuous network of CNTs between grains, a pronounced effect of CNT straightening before fracture was expected to show enhanced fracture toughening [9]. Study on $\mathrm{Si}_{3} \mathrm{~N}_{4}-\mathrm{Al}_{2} \mathrm{O}_{3}-\mathrm{Y}_{2} \mathrm{O}_{3}-\mathrm{CNT}$ nanocomposite showed improvement in modulus of elasticity by $15 \%$, whereas bending strength increased by $37 \%$ [21]. Peigney et al studied hot pressing of $\mathrm{MgAl}_{2} \mathrm{O}_{4}-\mathrm{CNT}$ composite with improper CNT dispersion and reinforcement effect was not to the order that was expected out of the system [31]. Ning et al stressed on the requirement of improvement in CNT dispersion to enhance the strengthening by CNTs [28].

Mechanical aspects of load transfer at CNT-matrix interface have been relatively poorly studied in these studies without detailed experimental evidence. Computational tools have been utilized in grasping a better idea of interfacial mechanism in CNT/ceramic system $[17,147,165,181-183]$. Table 2.9 is extracted from Table 2.8 as a subset presenting details of investigations on some of the CNT- ceramic composite system. Table 2.9 explains the processes involving high temperature sintering consolidation of various ceramic nanocomposites. Different degree of strengthening and fracture toughening were elicited such as poor cohesion between matrix and CNTs, straightening of CNTs and improved CNT dispersion. Since the direct attention is given to the $\mathrm{Al}_{2} \mathrm{O}_{3}-\mathrm{CNT}$ nanocomposites in the current work, detailed review on this system follows in the consequent section.

\subsection{Aluminum Oxide-CNT Nanocomposite}

Aluminum oxide-CNT nanocomposites for improved fracture toughness have been the focus of recent investigations $[31,37,184]$. Hot pressing, spark plasma 
Table 2.8: Evolution and development of CNT-reinforced ceramics by various processing techniques

\begin{tabular}{|c|c|c|c|c|c|c|c|c|c|c|c|c|c|c|c|c|c|c|c|c|c|}
\hline & \begin{tabular}{|l} 
JuL \\
1998 \\
\end{tabular} & $\begin{array}{l}\text { Oct. } \\
1999 \\
\end{array}$ & $\begin{array}{l}\text { Aup. } \\
2000\end{array}$ & $\begin{array}{l}\text { Aur. } \\
2001\end{array}$ & $\begin{array}{l}\text { May } \\
2002 \\
\end{array}$ & Dec. 2002 & $\begin{array}{l}\text { Feb. } \\
2003\end{array}$ & $\begin{array}{r}\text { May } \\
2003 \\
\end{array}$ & $\begin{array}{r}\text { Oct } \\
2003 \\
\end{array}$ & $\begin{array}{l}\text { Dec. } \\
2003 \\
\end{array}$ & $\begin{array}{l}\text { Feb. } \\
2004\end{array}$ & $\begin{array}{l}\text { JuL } \\
2004\end{array}$ & Dec. 2004 & $\begin{array}{l}\text { Jan. } \\
2005 \\
\end{array}$ & \begin{tabular}{|l|} 
Mar. \\
2005 \\
\end{tabular} & JuL 2005 & \begin{tabular}{|l|l|} 
Oct. \\
2005 \\
\end{tabular} & Jan. 2006 & Mar. 2006 & Auz. 2006 & $\begin{array}{l}\text { Jan. } \\
2007 \\
\end{array}$ \\
\hline $\begin{array}{c}\text { Hot } \\
\text { pressing }\end{array}$ & \begin{tabular}{|c|} 
Ma \\
CNT \\
SiC \\
China \\
$26]$ \\
\end{tabular} & $\begin{array}{c}\text { Peigney, } \\
\text { MWNTI' } \\
\text { Fe-Al } 2 \text {, } \\
\text { France } \\
{[31]} \\
\end{array}$ & $\begin{array}{c}\text { Siegel } \\
\text { MWNT/ } \\
\mathrm{Al}_{2} \mathrm{O}_{3} \\
\mathrm{RPI}[16]\end{array}$ & & & & 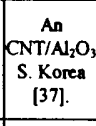 & & & & & & & & \begin{tabular}{|c|}
$\mathrm{Lim}[167]$ \\
$\mathrm{CNT} / \mathrm{Ad}_{2} \mathrm{O}_{3}$ \\
S. Korea
\end{tabular} & & \begin{tabular}{c|}
$\mathrm{Fan}$ \\
$\mathrm{CNT} / \mathrm{Al}_{2} \mathrm{O}_{3}$ \\
$\mathrm{Chins}[168]$
\end{tabular} & \begin{tabular}{|c|} 
Balasi [169] \\
Sis $N_{4}+C \mathrm{CNT}$ \\
Hungary \\
\end{tabular} & & \begin{tabular}{|c|} 
Macensin \\
{$[20] \mathrm{CNT} /$} \\
$\mathrm{Al} \mathrm{O}_{3} \mathrm{O}$ Thailend \\
$\mathrm{Ye}[170]$ China
\end{tabular} & \\
\hline $\begin{array}{l}\text { Hight Temp. } \\
\text { estrusion }\end{array}$ & & & & \begin{tabular}{|l|} 
Peigney \\
MWNT/ \\
FeAl $\mathrm{O}_{3}$ \\
France[9 \\
\end{tabular} & & & & & & & & & & & & & & & & $\begin{array}{c}\mathrm{CNT} / \mathrm{BaA} \mathrm{A}_{2} \mathrm{SiO}_{4} \\
\mathrm{Fan} \text { [155], } \\
\text { China } \\
\mathrm{CNT} / \mathrm{Al}_{2} \mathrm{O}_{3}\end{array}$ & \\
\hline \begin{tabular}{|c|} 
Spark \\
plasma \\
sintering \\
\end{tabular} & & & & & $\begin{array}{l}\text { Amiyg } \\
\text { MWNT/ } \\
\mathrm{Al}_{2} \mathrm{O}_{3}\end{array}$ & \begin{tabular}{|c|} 
Amiya \\
$\mathrm{CNT}_{\mathrm{AN}} \mathrm{Al}_{2} \mathrm{O}_{3}$ \\
$\mathrm{UC} \mathrm{Da} .[166]$ \\
\end{tabular} & & & & & \begin{tabular}{|c|} 
Thang \\
CNT/ WCCo \\
China 30$]$ \\
\end{tabular} & $\begin{array}{c}\text { Paddure } \\
\text { MWNT/A } / \mathrm{A}_{2} \mathrm{O}_{3} \\
\mathrm{U} \text {. Conn. [172] }\end{array}$ & \begin{tabular}{|c|} 
Balasi [173] \\
$\mathrm{Si}_{3} \mathrm{~N}_{4}+\mathrm{CNT}$ \\
Hungary \\
\end{tabular} & & & & & \begin{tabular}{|c|} 
Balasi [169] \\
$\mathrm{Si}_{3} \mathrm{~N}_{4}+\mathrm{CNT}$ \\
Hungary \\
\end{tabular} & \begin{tabular}{|c|} 
Balasi $[174]$ \\
$\mathrm{Si}_{3} \mathrm{~N}_{4}+\mathrm{CNT}$ \\
Hungary
\end{tabular} & & $\begin{array}{c}\text { Pednure [128] } \\
\text { CNT/ } \\
\mathrm{Al}_{2} \mathrm{O}_{3} \text { Othio } \\
\end{array}$ \\
\hline $\begin{array}{c}\text { Surfactant } \\
\text { anciated } \\
\text { processing }\end{array}$ & & & & & $\begin{array}{l}\text { UC Dav } \\
\text { [171] }\end{array}$ & & \begin{tabular}{|c|} 
Ning \\
CNT/SiO \\
China/28] \\
\end{tabular} & & & & & & & & & & & & & & \\
\hline $\begin{array}{c}\text { In-Situ } \\
\text { CVD }\end{array}$ & & & & & & & & \begin{tabular}{|l|}
$\mathrm{Xia} / 177$ \\
$\mathrm{~A}_{2} \mathrm{O}_{y} / \mathrm{CNT}$ \\
Brown $\mathrm{OR}$ \\
\end{tabular} & & & & & & & & & & & & & \\
\hline CCVD & & & & & & & & & RulPeigney & & & & & & \begin{tabular}{|c|} 
Mill175] \\
CNT/A2O3 \\
Chin\&/Tempe
\end{tabular} & & & & & & \\
\hline $\begin{array}{c}\text { Hot } \\
\text { Lostatic } \\
\text { Pressing }\end{array}$ & & & & & & & & & $\begin{array}{l}\mathrm{CNT} / \mathrm{MgAl}_{2} \mathrm{O} \\
{ }_{4} \text { France [29] }\end{array}$ & \begin{tabular}{|c|} 
Balazsi \\
CNT/ $\mathrm{Si}_{3} \mathrm{~N}$ \\
Hungary \\
{$[21]$}
\end{tabular} & & & & & & & & & & & \\
\hline Sol Ged & & & & & & & & & & & \begin{tabular}{|c|} 
Jitianu \\
CNT/TiO \\
France [23] \\
\end{tabular} & & \begin{tabular}{|c|}
$\mathrm{Mo}$ \\
$\mathrm{CNT}-\mathrm{Al}_{2} \mathrm{O}_{3}$ \\
Korea [176]
\end{tabular} & & & & & & & & \\
\hline $\begin{array}{l}\text { Colloidal } \\
\text { processing }\end{array}$ & & & & & & 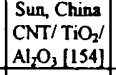 & & & & & & & & \begin{tabular}{|c|} 
Sun [11] \\
$\mathrm{A}_{2} \mathrm{O} / \mathrm{CNT}$ \\
China \\
\end{tabular} & & & & & & & \\
\hline $\begin{array}{c}\text { Molecular } \\
\text { kevel mixing }\end{array}$ & & & & & & & & & & & & & & & & $\begin{array}{c}\mathrm{Cha}[177] \\
\mathrm{CNT} / \mathrm{Al}_{2} \mathrm{O}_{3} \\
\text { Korea }\end{array}$ & & & & & \\
\hline \begin{tabular}{|c|} 
Thermal \\
CVD/Spray \\
Pyrohysis \\
\end{tabular} & & & & & & \begin{tabular}{|c|}
$\mathrm{Bai}$ \\
$\mathrm{Al}_{2} \mathrm{O} / \mathrm{CNT}$ \\
France [178]
\end{tabular} & & & Grobert & & & & & & & & & & & & \\
\hline Anodization & & & & & & & & & $\begin{array}{c}\text { MPP, } \\
\text { Stuttagart }\end{array}$ & & & & & & & & & & \begin{tabular}{c|} 
Wen \\
CNT/ \\
Korea $[1800]$ \\
Kons
\end{tabular} & & \\
\hline $\begin{array}{l}\text { Liquid } \\
\text { Precursor }\end{array}$ & & & & & & & & & & & & & & & & & \begin{tabular}{|c|} 
Katsuda \\
CNT/SiCN \\
Japan[4]
\end{tabular} & & & & \\
\hline
\end{tabular}


sintering, sol-gel synthesis, surfactant assisted processing, Catalytic CVD, hot isostatic pressing, molecular level mixing, spray pyrolysis, high temperature extrusion, liquid precursor spraying, colloidal processing, etc, have been used to fabricate $\mathrm{Al}_{2} \mathrm{O}_{3}$-CNT nanocomposite $[16,17,37,128,154,155,166-168,171,172$, 177, 178, 180]. Siegel et al were the pioneers in processing $\mathrm{Al}_{2} \mathrm{O}_{3}-\mathrm{CNT}$ nanocomposite and utilized hot pressing as consolidation technique [16]. Improvement of upto $24 \%$ in fracture toughness was achieved in $\mathrm{Al}_{2} \mathrm{O}_{3}-\mathrm{CNT}$ nanocomposite [16]. Zhan et al. adopted spark plasma sintering and evinced tremendous improvement in the electrical conductivity (from $10^{-12}$ to $1050 \mathrm{~S} / \mathrm{m}$ ) and fracture toughness (by three times) of $\mathrm{Al}_{2} \mathrm{O}_{3}$-CNT nanocomposite [185]. Wang et al adopted the similar methodology as that of Zhan et al., but did not observe the similar enhancement in fracture toughness [172]. Damage tolerance of $\mathrm{Al}_{2} \mathrm{O}_{3}-\mathrm{CNT}$ nanocomposite led to absence of Vicker indentation cracks [172]. Disparity of results were attributed to dissimilar densities and residual compressive stresses in the $\mathrm{Al}_{2} \mathrm{O}_{3}-\mathrm{CNT}$ nanocomposite [186]. Mo et al observed toughening of $\mathrm{Al}_{2} \mathrm{O}_{3}-\mathrm{CNT}$ nanocomposite by crack bridging [176]. An outlook and description of $\mathrm{Al}_{2} \mathrm{O}_{3}-\mathrm{CNT}$ nanocomposite by various researchers is presented in Table 2.10 .

Effort of CNT dispersion by colloidal processing and consolidation by hot pressing was studied by Sun et al [11]. CNT pullout was observed in the fractured surface of the $\mathrm{Al}_{2} \mathrm{O}_{3}$-CNT composite, Fig. 2.22. Hot pressed $\mathrm{Al}_{2} \mathrm{O}_{3}$-CNT nanocomposite displayed $10 \%$ improvement (from 496 to $554 \mathrm{MPa}$ ) in the bend 
Table 2.9: Description of ceramic-CNT nanocomposites research

\begin{tabular}{|c|c|c|c|c|}
\hline & $\begin{array}{c}\text { Peigney } \\
\text { (Univsite Paul-Sabatier, } \\
\text { France) }[9[\end{array}$ & \begin{tabular}{l}
\multicolumn{1}{c}{ Balazsi } \\
(Hungarian Academy of Sciences, \\
Hungary) $[21]$
\end{tabular} & $\begin{array}{c}\text { Peigney } \\
\text { (Univsite Paul- } \\
\text { Sabatier, } \\
\text { France) [31] }\end{array}$ & $\begin{array}{c}\text { Ning } \\
\text { (Chinese Academy of Sciences, } \\
\text { China) }[28]\end{array}$ \\
\hline Ceramic & $\mathrm{Fe}-\mathrm{Al}_{2} \mathrm{O}_{3}$ & $\mathrm{Si}_{3} \mathrm{~N}_{4} 90 \%-\mathrm{Al}_{2} \mathrm{O}_{3} 4 \%-\mathrm{Y}_{2} \mathrm{O}_{3}-6 \%$ & $\mathrm{Mg} \mathrm{Al}_{2} \mathrm{O}_{4}$ & $\mathrm{SiO}_{2}$ \\
\hline CNT & $\begin{array}{c}\text { Arc Discharge (SWNT + } \\
\text { MWNT) } 1.5-15 \mathrm{~nm} \text {. Mostly 2-3 } \\
\text { walls CNT (Grown in-situ) }\end{array}$ & $\begin{array}{l}\text { catalytic decomposition } \\
\text { of acetylene on aluminum oxide } \\
\text { supported } \mathrm{Co} / \mathrm{Fe} \text { catalyst }\end{array}$ & $\begin{array}{l}\text { SWNT (CCVD) : } \\
\text { In-situ }\end{array}$ & Catayltic pyrolysis of hydrocarbon \\
\hline $\begin{array}{l}\text { Mixing/ } \\
\text { Processing } \\
\text { Method }\end{array}$ & $\begin{array}{l}\text { Hot Pressing }(1475 \mathrm{C}) \text { in vacuum } \\
\text { (Thermal treatment optimization) }\end{array}$ & $\begin{array}{l}\text { Ball-milled in ethanol (several hours), } \\
\text { ultrasonicated, } \\
\text { Pressing: } 220 \mathrm{MPa} \text {, Sinter } 1700^{\circ} \mathrm{C}\end{array}$ & $\begin{array}{l}\text { Hot Pressing } \\
\left(1475^{\circ} \mathrm{C}\right)\end{array}$ & $\begin{array}{c}\text { CNT dispersed in alcohol, }+ \\
\text { ultrasonification. Gel was washed } \\
\text { dried and calcined }\left(500^{\circ} \mathrm{C}\right) \text {. Sinter } \\
\left(1300^{\circ} \mathrm{C}, 25 \mathrm{MPa}\right) \text { in } \mathrm{N}_{2} \text { atmosphere }\end{array}$ \\
\hline Measurement & $\begin{array}{l}\text { Surface Area to calculate CNT } \\
\text { content }\end{array}$ & $\begin{array}{c}\text { Modulus of Elasticity, Bending } \\
\text { Strength }\end{array}$ & $\begin{array}{l}\text { conductivity } \\
\text { studies }\end{array}$ & $\begin{array}{l}\text { Surfactants role in aiding } \mathrm{CNT} / \mathrm{SiO}_{2} \\
\text { composite fabrication }\end{array}$ \\
\hline $\begin{array}{l}\text { Fracture } \\
\text { Toughness }\end{array}$ & $1.8 \times 1.8 \times 16 \mathrm{~mm}$ specimen & $\begin{array}{c}\text { Not done } \\
\text { (Sample: } 3.5 \times 5 \times 50 \mathrm{~mm})\end{array}$ & Not done & $\begin{array}{c}3 \times 4 \times 35 \mathrm{~mm} \\
5 \text { vol\% CNT }+ \text { Surf. } \sim 146 \% \text { impr. }\end{array}$ \\
\hline Key Results & $\begin{array}{l}1050^{\circ} \mathrm{C}, 6 \mathrm{~min} \text { thermal treatment } \\
\text { found optimum cohesion } \\
\text { between } \mathrm{CNT} \text { and matrix is poor. }\end{array}$ & $\begin{array}{c}\text { Deterioration of carbon fibers (ref. } \\
\text { Sample) was observed } 15-37 \% \\
\text { improvement in mech. properties. } \\
\text { Pressure, sintering time and } \\
\text { temperature resulted disappearance of } \\
\text { MWNT }\end{array}$ & $\begin{array}{l}\text { continuous } \\
\text { network of CNT } \\
\text { between oxide } \\
\text { grains } \\
\text { Straightening of } \\
\text { CNT before } \\
\text { fracture }\end{array}$ & $\begin{array}{c}\text { Better dispersion of CNT has more } \\
\text { interfaces } \rightarrow \text { can strongly absorb crack } \\
\text { energy } \\
\text {-enhanced bending strength and } \\
\text { fracture toughness }\end{array}$ \\
\hline Other & $\begin{array}{l}\text { Quality and Quantity defined by } \\
\text { author for defining CNT }\end{array}$ & $\begin{array}{c}\text { Better to shorten sintering time and } \\
\text { pressure. }\end{array}$ & $\begin{array}{l}\text { Increased electrical } \\
\text { conductivity with } \\
\text { CNT }\end{array}$ & $\begin{array}{l}\text { How interface acts as booster } \\
\text { interface chemistry: future }\end{array}$ \\
\hline
\end{tabular}


Table 2.10: Description of $\mathrm{Al}_{2} \mathrm{O}_{3}-\mathrm{CNT}$ nanocomposites research

\begin{tabular}{|c|c|c|c|c|}
\hline & $\begin{array}{c}\text { Amiya } \\
\text { (UC Davis) }[186]\end{array}$ & $\begin{array}{c}\text { Siegel } \\
(\text { RPI, New York) [16] }\end{array}$ & $\begin{array}{c}\text { Padture, Tanaka } \\
\text { (U.Connecticut, CT) } \\
\text { (NIMS, Japan) [172] }\end{array}$ & $\begin{array}{c}\text { Mo } \\
\text { (KAIST, Korea) [176] }\end{array}$ \\
\hline $\begin{array}{l}\mathrm{Al}_{2} \mathrm{O}_{3} \text { Powder } / \\
\text { Vendor } / \\
\text { Morphology }\end{array}$ & $\mid \begin{array}{c}(80 \% \alpha+20 \% \gamma) \mathrm{Al}_{2} \mathrm{O}_{3} \\
300 \mathrm{~nm}(40 \mathrm{~nm} \text { Crystallite }) \text { and } \\
20 \mathrm{~nm}: \text { Irregular }\end{array}$ & $\begin{array}{l}\text { Nanophase Tech. }(39 \mathrm{~nm} \\
\text { average diameter) }\end{array}$ & $\begin{array}{c}\text { Baikowski Intl }(80 \% \alpha+20 \% \gamma) \\
\mathrm{Al}_{2} \mathrm{O}_{3} \\
300 \mathrm{~nm}(40 \mathrm{~nm} \text { Crystallite) and } 20 \\
\text { nm: Irregular }\end{array}$ & Al-tri-sec-butoxide \\
\hline SWNT/ Vendor & $\begin{array}{l}\text { Carbon Nanotechnology } \\
\text { (HIPco process) }\end{array}$ & $\begin{array}{l}\text { MWNT: Electric Discharge } \\
\text { method/ Oxidation } \sim 640^{\circ} \mathrm{C}\end{array}$ & $\begin{array}{c}\text { Carbon Nanotechnology (Same } \\
\text { as Zhan) }\end{array}$ & $\begin{array}{c}\text { MWNT: } 10-30 \mathrm{~nm} ; 10-50 \mu \mathrm{m} \\
\text { CVD:aluminum oxide supported }\end{array}$ \\
\hline $\begin{array}{c}\text { Mixing/ } \\
\text { Processing Method }\end{array}$ & $\begin{array}{l}\text { Ethanol + ultrasonic agitation } \\
\text { Ball Milling }\left(\mathrm{ZrO}_{2} \text { ball/ 24h }\right) / \\
\mathrm{SPS}\end{array}$ & $\begin{array}{c}\text { Ethanol }+ \text { ultrasonic } \\
\text { agitation, dried and crushed/ } \\
\text { Hot Pressed at T: } 1300^{\circ} \mathrm{C} \\
\text { lh, } 60 \mathrm{MPa})\end{array}$ & $\begin{array}{c}\text { Methanol }+ \text { ultrasonic agitation } / \\
\text { Ball Milling }\left(\mathrm{ZrO}_{2} \text { ball } / 24 \mathrm{~h}\right) / \\
\mathrm{SPS}\end{array}$ & $\begin{array}{c}\text { CNT dispersed in Ethanol + Aluminum } \\
\text { oxide sol (Yolda's Process) Hydrolysis } \\
\text { and Peptization of Al-hydroxide } \\
(\mathrm{AlOOH}) \text {. SPS }\left(1650^{\circ} \mathrm{C}, 5 \mathrm{~min}\right) \text {; sintered } \\
\text { at } 1000 \mathrm{C}, 6 \mathrm{~h}\end{array}$ \\
\hline $\begin{array}{c}\text { Electrical } \\
\text { Conductivity } \\
(\mathrm{S} / \mathrm{m}) / \text { Density } \\
(\mathrm{g} / \mathrm{cc}) \\
\end{array}$ & $\begin{array}{c}\mathrm{Al}_{2} \mathrm{O}_{3}: 10^{-10}-10^{-12} 10 \text { vol. } \% \\
\text { SWNT: } 1510 \mathrm{~S} / \mathrm{m}\end{array}$ & $\begin{array}{l}\mathrm{Al}_{2} \mathrm{O}_{3}+4 \text { vol. } \% \mathrm{CNT} \\
\text { : Best Wear Resistance }\end{array}$ & $\begin{array}{c}\mathrm{Al}_{2} \mathrm{O}_{3}: 3.898 \mathrm{~g} / \mathrm{cc} \\
10 \text { vol. } \% \text { SWNT: } 3.568 \mathrm{~g} / \mathrm{cc}\end{array}$ & $\begin{array}{c}\text { Drying } 350^{\circ} \mathrm{C}, 6 \mathrm{~h} ; \text { calcination } 1250^{\circ} \mathrm{C}, \\
1 \mathrm{~h}\end{array}$ \\
\hline $\begin{array}{c}\text { Strength/Fr. } \\
\text { Tough. }\left(\mathrm{MPa} \mathrm{m}{ }^{1 / 2)}\right.\end{array}$ & $\begin{array}{c}\mathrm{Al}_{2} \mathrm{O}_{3}: 3.33 \\
10 \text { vol \% SWNT: } 9.7\end{array}$ & $\begin{array}{c}\mathrm{Al}_{2} \mathrm{O}_{3}: 3.4 \\
10 \text { vol\% } \mathrm{MWNT}: 4.2(5 \mathrm{~kg})\end{array}$ & $\begin{array}{c}\mathrm{Al}_{2} \mathrm{O}_{3}: 3.22 \\
10 \text { vol.\% SWNT: } 3.32\end{array}$ & $\begin{array}{l}\mathrm{Al}_{2} \mathrm{O}_{3}: 1.0 \text { (Normalized) } \\
1.5 \text { vol\% MWNT }: 1.1\end{array}$ \\
\hline Key Results & $\begin{array}{c}\text { No Vicker cracking } \\
\text { Highest Indentation toughness } \\
\text { at } \mathrm{Al}_{2} \mathrm{O}_{3} / 10 \text { vol. \% CNT } \\
\text { Significant inc. in Electrical } \\
\text { conductivity }\end{array}$ & $\begin{array}{c}24 \% \text { Increase in } \mathrm{Fr} \text {. } \\
\text { Toughness }\left(\mathrm{Al}_{2} \mathrm{O}_{3}+10 \text { vol }\right. \\
\% \mathrm{MWNT}) \\
\text { Damage delocalization (well } \\
\text { dispersed MWNT) }\end{array}$ & $\begin{array}{l}\text {-Enhanced contact damage } \\
\text { resistance }\end{array}$ & $\begin{array}{c}\text { toughening of } \mathrm{CNT} \text { reinforced aluminum } \\
\text { oxide } \\
\text { crack bridging effect of } \mathrm{CNT}\end{array}$ \\
\hline Other & $\begin{array}{l}\text { Did not present toughening } \\
\text { mechanism }\end{array}$ & $\begin{array}{c}\text { No discussion on interfacial } \\
\text { properties }\end{array}$ & $\begin{array}{c}\text { Clear explanation for the result is } \\
\text { missing }\end{array}$ & $\begin{array}{l}\text { Does not includes discussion on the } \\
\text { bonding of CNT with matrix }\end{array}$ \\
\hline
\end{tabular}


strength [11]. Only marginal improvement from 3.9 to $4.0 \mathrm{MPa} \mathrm{m}^{1 / 2}$ was observed in the fracture toughness of the nanocomposite [11]. Marginal improvement in the toughness is attributed to damage of CNTs at high temperature processing as intimated by Peigney et al [9]. In addition, low relative density $(\sim 85-95 \%)$ possibly reduced the facture toughness of hot-pressed nanocomposites.

High temperature extrusion $\left(1500^{\circ} \mathrm{C}\right.$ at $\left.43 \mathrm{MPa}\right)$ elicited retention of undamaged CNTs in the CNT-Fe- $\mathrm{Al}_{2} \mathrm{O}_{3}$ and $\mathrm{CNT}-\mathrm{Fe} / \mathrm{Co}-\mathrm{MgAl}_{2} \mathrm{O}_{4}$ composite, respectively (Fig. $2.23 \mathrm{a}$ and b) [9]. This effort was directed for adjusting the electrical conductivity value in the composite [9]. Higher electrical conductivity $(\sim 20 \mathrm{~S} / \mathrm{m})$ was observed along extrusion direction, whereas it dropped to $0.6 \mathrm{~S} / \mathrm{m}$ along transverse direction. Alignment of CNTs was responsible towards imparting such a difference in the electrical conductivity [9]. Presence of CNTs also assisted in restricting grain growth and serving as lubricating agent during hot extrusion. No mechanical property data was discussed in the paper, but retention of undamaged CNTs and CNT alignment via extrusion insinuates possible anisotropic property enhancement [9].

With many publications in processing nanocrystalline aluminum oxide, Zhan et al, and Duan et al demonstrated improved fracture toughness, of upto three times than that of aluminum oxide, using spark plasma sintering consolidation of $\mathrm{CNT}-\mathrm{Al}_{2} \mathrm{O}_{3}$ composite, Fig $2.24[125,166,171,186-191]$. However, the primary interest of Zhan et 


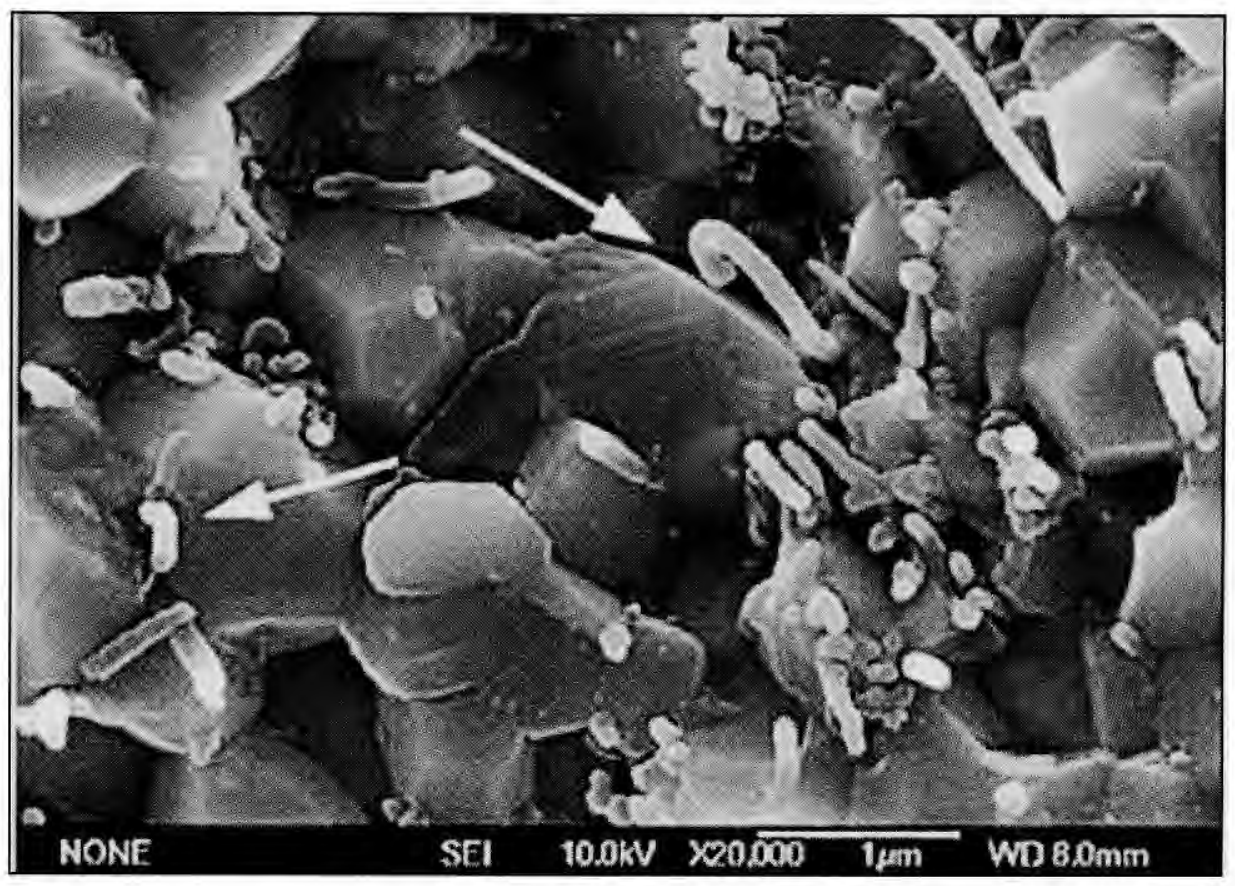

Fig. 2.22: SEM of the fractured surface of $\mathrm{Al}_{2} \mathrm{O}_{3}-\mathrm{CNT}$ composite sintered at $1450^{\circ} \mathrm{C}$ showing CNT pullout [11].

al was also concentrated on enhancing electrical properties using CNTs in the aluminum oxide matrix [166]. Electrical conductivity enhancement from $10^{-10} \mathrm{~S} / \mathrm{m}$ to $3345 \mathrm{~S} / \mathrm{m}$ was observed in 15 vol. \% CNT- $\mathrm{Al}_{2} \mathrm{O}_{3}$ nanocomposite. This conductivity increase was because of CNT addition, and formation of CNT-ropes along grain boundaries creating network of electrically conductive paths [166]. Dispersion of CNTs along $\mathrm{Al}_{2} \mathrm{O}_{3}$ grains is shown in Fig. 2.25 [166]. Later, Wang et al repeated the methodology of the Zhan et al and showed that toughness of $\mathrm{CNT}-\mathrm{Al}_{2} \mathrm{O}_{3}$ matrix was not improved [172]. The 

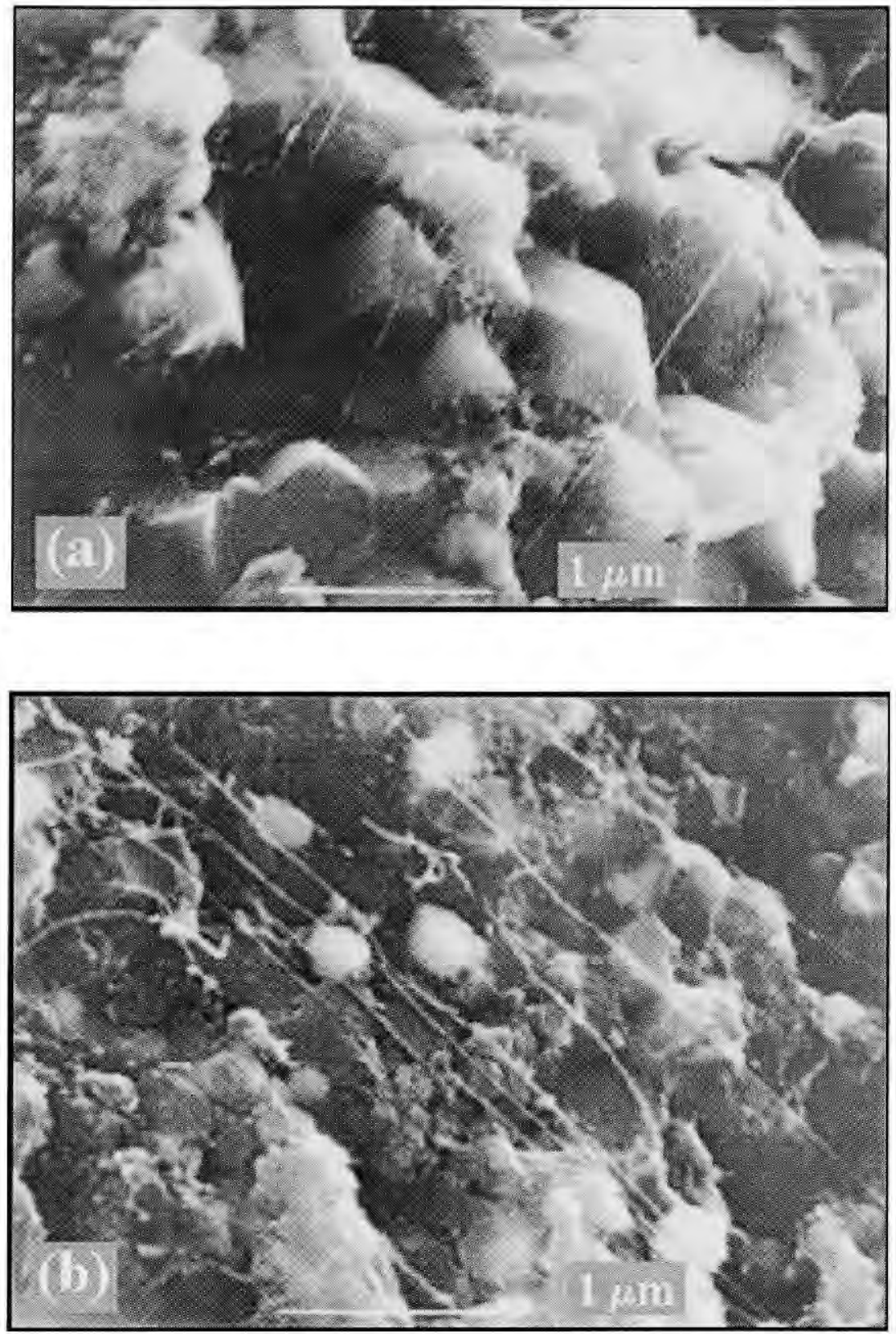

Fig. 2.23: SEM images of fracture profiles of the CNT-Fe- $\mathrm{Al}_{2} \mathrm{O}_{3}$ (a) and $\mathrm{CNT}-\mathrm{Fe} / \mathrm{Co}-$ $\mathrm{MgAl}_{2} \mathrm{O}_{4}$ (b) composites prepared by extrusion at $1500^{\circ} \mathrm{C}$. Note the alignment of the CNTs [9]. 
discrepancy in data was because Zhan et al had used indentation methods to calculate fracture toughness, whereas the $\mathrm{Al}_{2} \mathrm{O}_{3}-\mathrm{CNT}$ composite shows contact-damage resistance, which was tracked through Hertzian indentation [172]. This unusual mechanical behavior was attributed to shear-deformable nature of CNTs in redistributing stress field upon indentation. Also, different densification achieved by Zhan (> 99\%) and Wang ( 95\%) induced discrepancy [185]. Generation of crack was limited by high porosity in Wang's

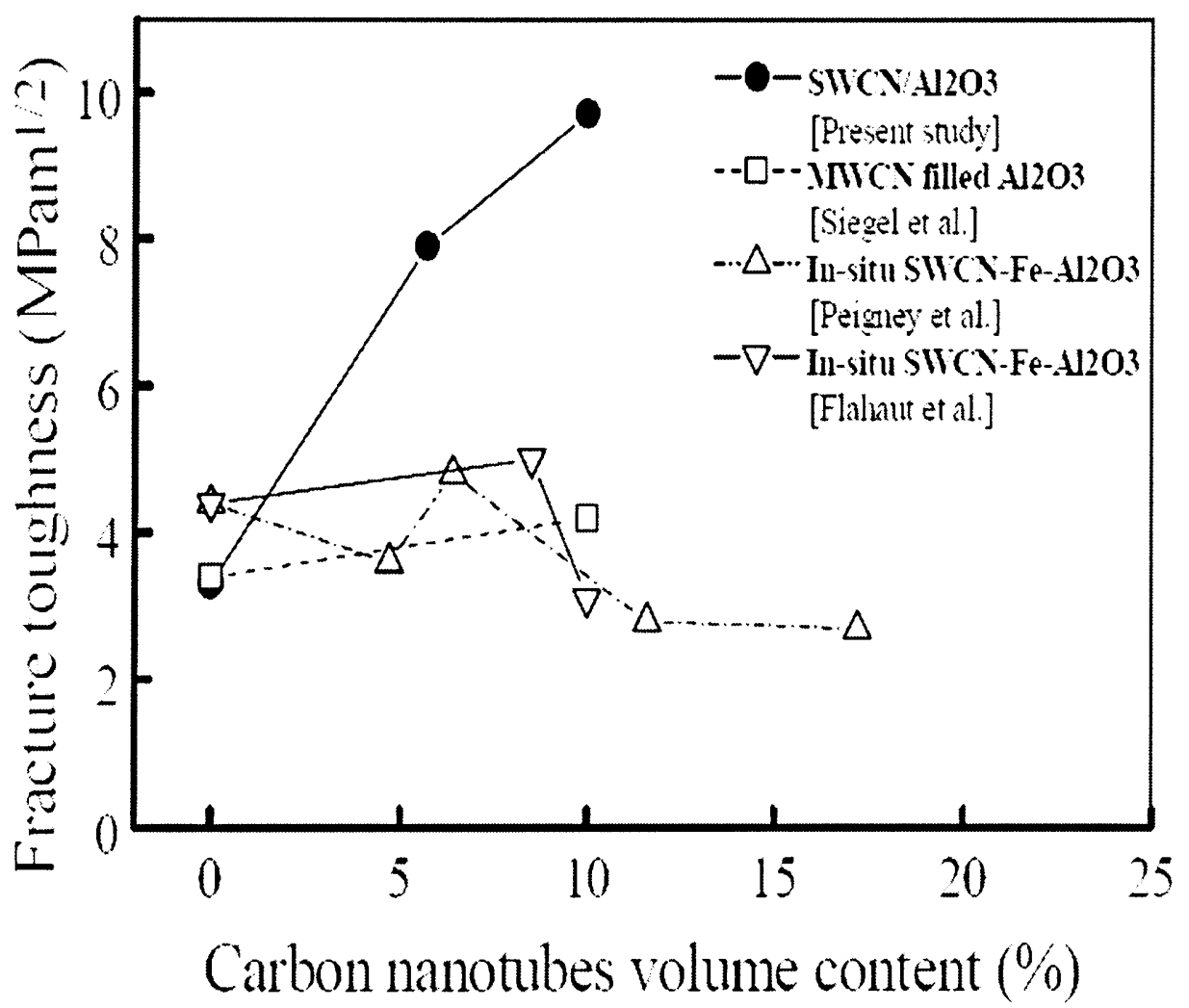

Fig. 2.24: Fracture toughness versus carbon nanotube volume content in aluminum oxide based composites as reported in literature[166]. (SWCNT: Single walled CNT). 

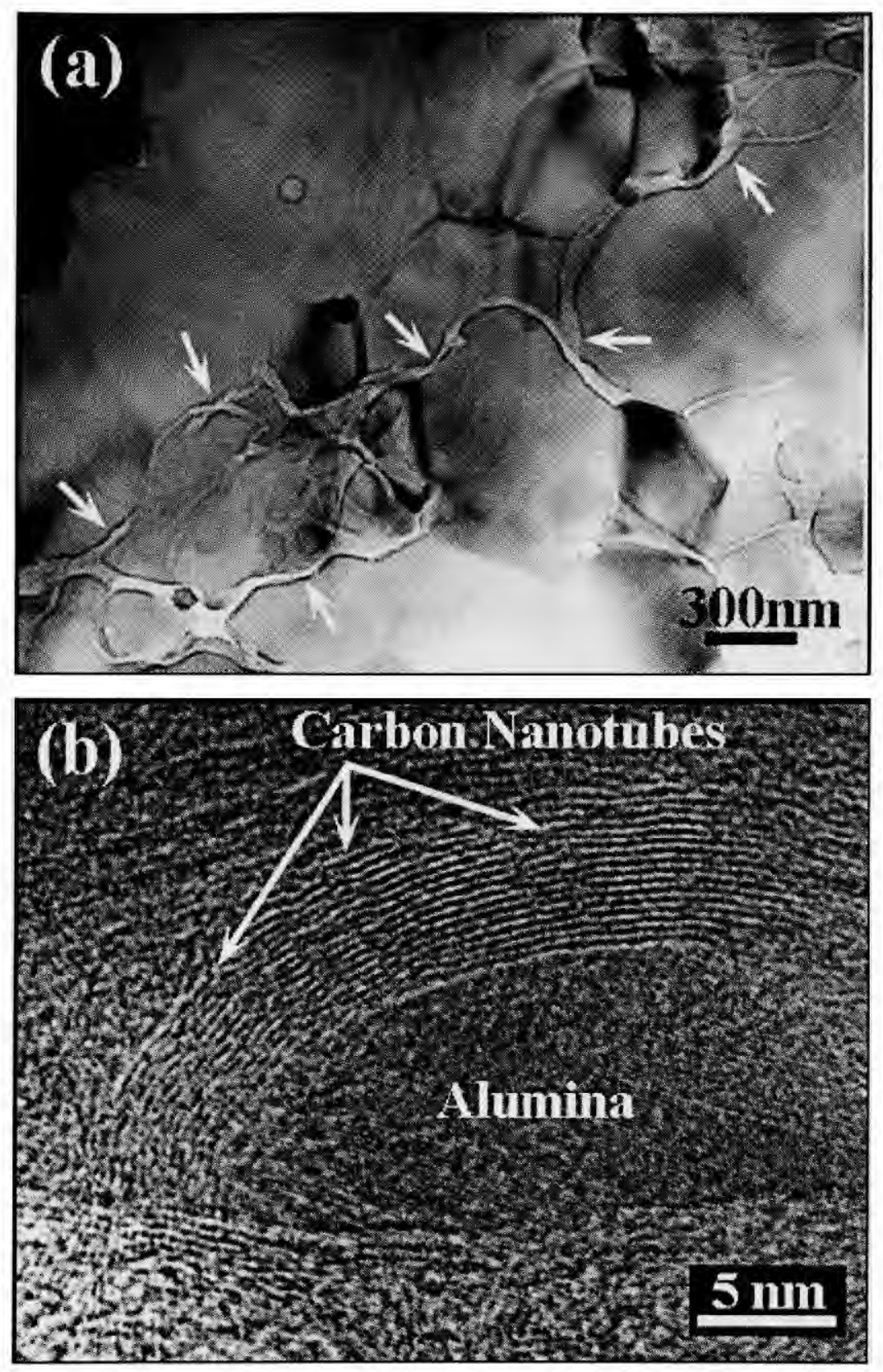

Fig. 2.25: TEM Micrographs of 5.7 vol. $\% \mathrm{SWCN} / \mathrm{Al}_{2} \mathrm{O}_{3}$ nanocomposite. (a) Bright-field

TEM image and (b) high-resolution TEM image of specimens in the fully dense 5.7 vol.\% SWCN $/ \mathrm{Al}_{2} \mathrm{O}_{3}$ nanocomposite. The arrows indicate the SWCN phase [166]. (SWCNT: Single walled CNT). 
work, since indentation initiated collapse of crack before the induction of plastic deformation in the material. On the other hand, Vicker indentation cracks were clearly observed in Zhan's paper. Moreover, high residual compressive stress $(2.0 \mathrm{GPa})$ in the alumina matrix, crack bridging, fiber pull-out and crack deflection were responisible for improved fracture toughness of spark plasma sintered $\mathrm{CNT}-\mathrm{Al}_{2} \mathrm{O}_{3}$ nanocomposite.

Surface assisted processing of CNTs followed by hot pressing (25 MPa at 1300 ${ }^{0} \mathrm{C}$ ) helped increasing the bending strength (from 51.5 to $97.0 \mathrm{MPa}$ ) and fracture toughness (from 1.0 to $2.46 \mathrm{MPa} \mathrm{m}^{1 / 2}$ ) of $\mathrm{SiO}_{2}$ ceramic [28]. CVD processing of CNT$\mathrm{Al}_{2} \mathrm{O}_{3}$ composite was elucidated by Xia et al along with computational modeling of CNT$\mathrm{Al}_{2} \mathrm{O}_{3}$ interface demonstrating CNT pullout, Fig. 2.26a, and crack deflection at CNT$\mathrm{Al}_{2} \mathrm{O}_{3}$ interface, Fig. 2.26b [17, 178]. Defects, residual stresses, alignment of crack path, and deflection of CNTs were stressed upon by researchers towards estimating the fracture toughness of the composite [17].

High aspect ratio of CNT diameter caused severe agglomeration. Hence a new technique for CNT dispersion was required. Catalytic CVD method was adopted by Rul et al for dispersing CNTs in $\mathrm{MgAl}_{2} \mathrm{O}_{4}$ matrix [29]. Insitu CCVD synthesis followed by hot pressing at $1300^{\circ} \mathrm{C}\left(43 \mathrm{MPa}\right.$ in vacuum of $10^{-4}$ torr $)$ retained the CNT distribution in the nanocomposite. CNTs inhibited grain growth and improved the electrical conductivity by seven orders of magnitude (from $10^{-10}$ to $4 \times 10^{-3} \mathrm{~S} / \mathrm{cm}$ ) [29]. Variety of 


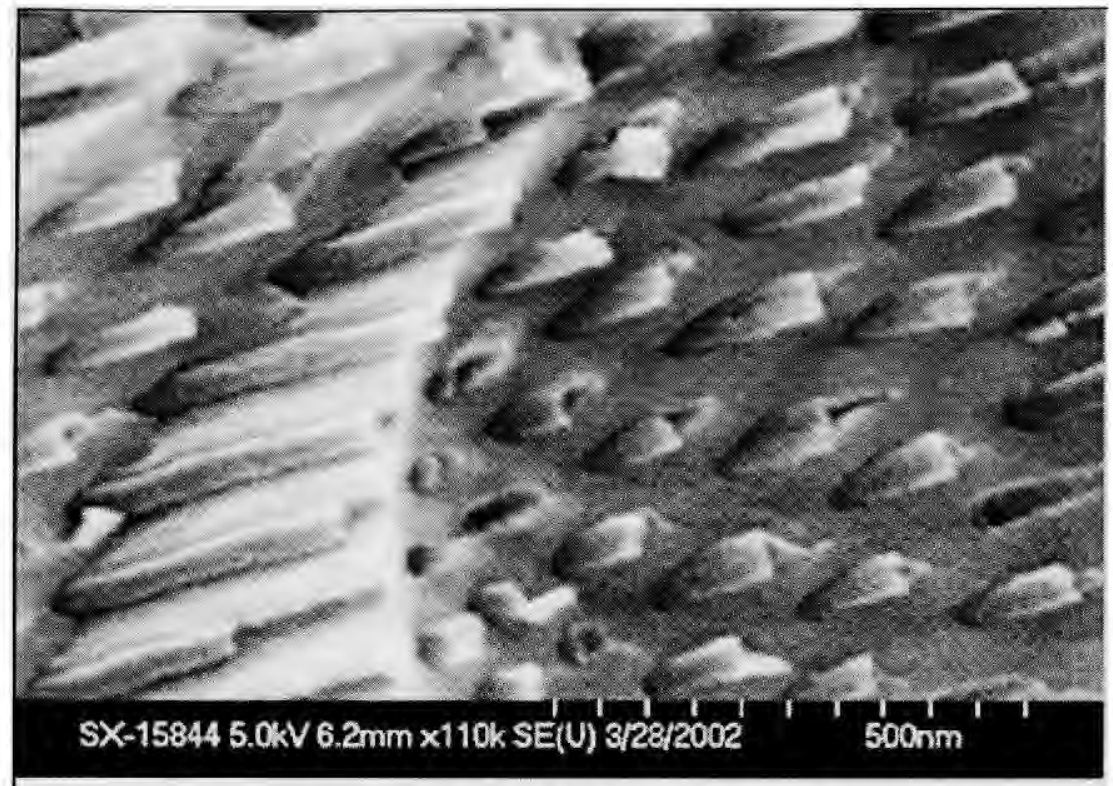

(a)

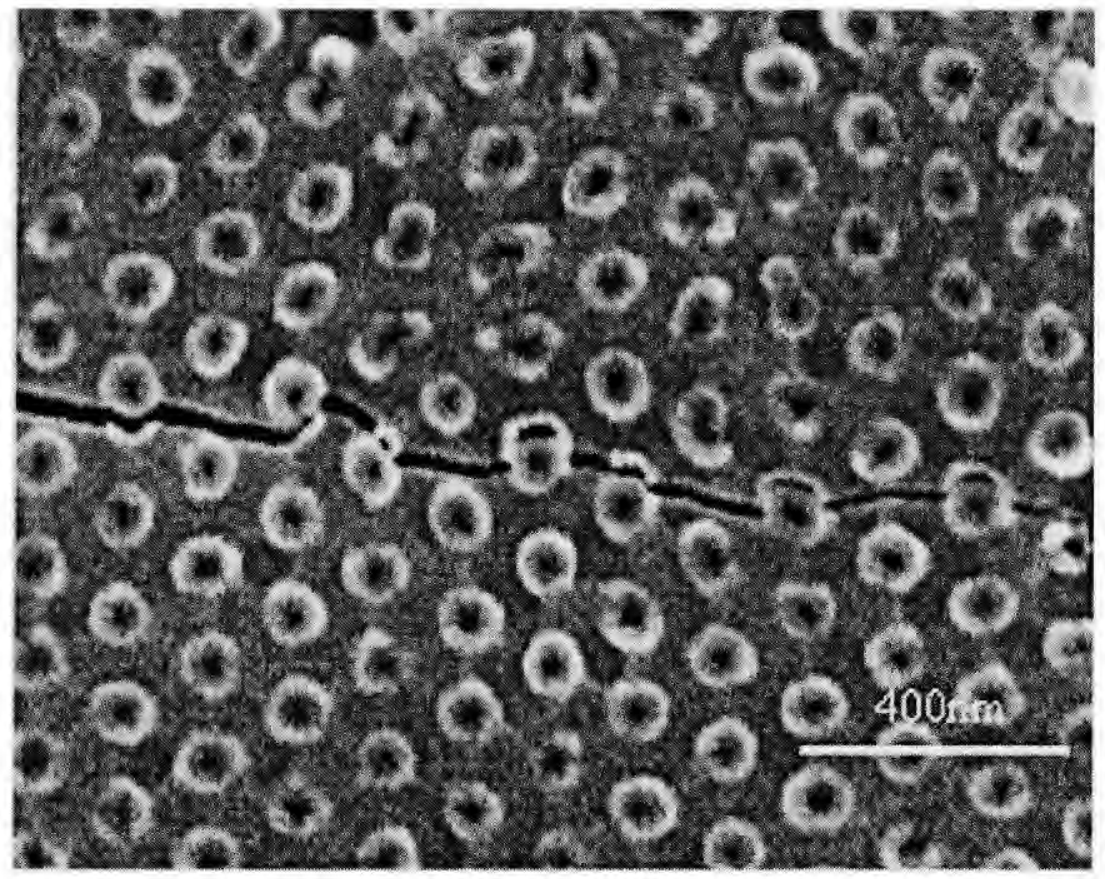

(b)

Fig. 2.26: a) SEM photograph of a fracture surface of the $20 \mu \mathrm{m}$-thick sample demonstrating CNT pullout, longitudinal delamination, and residual holes; (b) crack deflection at CNT along interface [17]. 
samples were produced, out of which the nomenclature of the samples $\mathrm{CMH}, \mathrm{C} 5, \mathrm{C10}$, CM2, and CM12 is presented in Table 2.11 A very well distributed network of CNTs was observed in the composite powders. Increased CNT content resulted restricted full densification, which was attributed to grain growth inhibition by CNTs. Fine grained matrix and widely interconnected CNT network was depicted in the fractured surface of nanocomposite. Fractured surface illustrated straightening of CNTs before breaking during the fracture, Fig. 2.27, which might show enhanced fracture toughness because of CNT pullout and CNT bridging [29].

Spray pyrolysis emerged as in-situ technique for fabrication of aluminum oxideCNT composites [179]. Here crack deflection and nanotube debonding served as site for fracture energy dissipation [179]. Molecular level mixing utilized functionalization of CNTs reacting with metal ions in solution [177]. Hardness of the $\mathrm{Al}_{2} \mathrm{O}_{3}-\mathrm{CNT}$ nanocomposite improved from $1500 \mathrm{H}_{\mathrm{V}}$ to $1700 \mathrm{H}_{\mathrm{V}}$. Addition and dispersion of functionalized CNTs was responsible for strengthening and toughening enhancement of upto $25 \%$ and $15 \%$ respectively for $\mathrm{CNT}-\mathrm{Al}_{2} \mathrm{O}_{3}$ nanocomposite [177]. CNT pullout and CNT bridging was also observed in the fractured surface with most of CNTs residing at grain boundary. Hence, CNT reinforced the matrix via load sharing and bridging mechanism [177]. Chemical bonding was observed between CNT and amorphous $\mathrm{Al}_{2} \mathrm{O}_{3}$ matrix, which imparted such increase in mechanical properties. 
Table 2.11: Nomenclature for powders and hot-pressed $\mathrm{MgAl}_{2} \mathrm{O}_{4}$ samples

\begin{tabular}{|c|c|c|c|c|c|c|}
\hline Sample & $\begin{array}{c}\text { Co } \\
(\mathbf{w t .} \%)\end{array}$ & $\begin{array}{c}\text { Mo } \\
(\mathbf{w t} \%)\end{array}$ & Preparation Mode & $\begin{array}{c}\text { CNT } \\
\text { content } \\
(\mathbf{w t . \%})\end{array}$ & $\begin{array}{c}\text { Density } \\
(\%)\end{array}$ & $\begin{array}{c}\text { Conductivity } \\
\text { (S/cm) }\end{array}$ \\
\hline CMH & 20 & 10 & Powder (Urea) & 0 & 97.3 & $10^{-10}$ \\
\hline C5 & 1 & 0 & Powder (Urea) & 0.55 & 100 & 0.0072 \\
\hline C10 & 20 & 0 & Gelcasting Foam (Urea) & 3.70 & 92.7 & 0.530 \\
\hline CM2 & 20 & 10 & Powder (Urea) & 5.10 & 83.8 & 1.00 \\
\hline CM12 & 10 & 5 & $\begin{array}{c}\text { Gelcasting Foam (with } \\
\text { Citric Acid) }\end{array}$ & 12.20 & 68.0 & 8.53 \\
\hline
\end{tabular}

Sol-gel technique was utilized to counteract the van der Waals attractive force between CNTs in the gel network. Sol-gel techniques depicted homogeneous dispersion in the aluminum oxide matrix without agglomeration, Fig. 2.28 [176]. The process was followed by spark plasma sintering to consolidate the $\mathrm{Al}_{2} \mathrm{O}_{3}-\mathrm{CNT}$ composite. Strong CNT bonding with matrix as CNT pullout was observed in the fractured surface indicating significant stress transfer from matrix to CNT. Enhanced density $(>99.5 \%)$, significant load transfer between matrix to CNTs, and bridging effect of CNTs during crack propagation were reasoned for enhanced fracture toughness (by $10 \%$ for $1.5 \mathrm{vol} . \%$ $\mathrm{CNT}$ ) of the $\mathrm{Al}_{2} \mathrm{O}_{3}-\mathrm{CNT}$ nanocomposite [176]. 


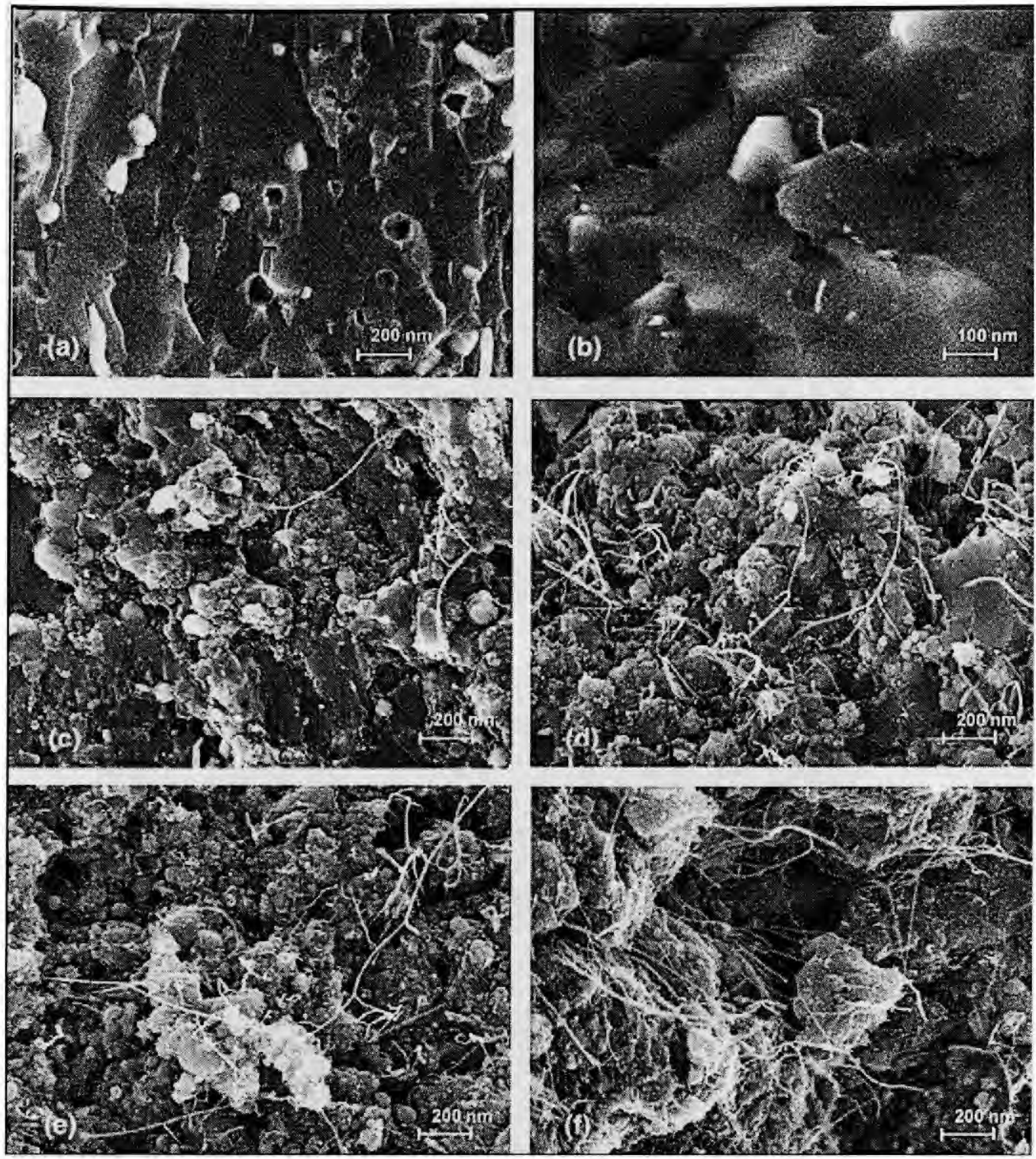

Fig. 2.27: High resolution SEM images of the fracture surface of the hot-pressed composites: (a) Sample without CNT showing mainly transgranular fracture. (b)-(e) Samples C5, C10, CM2 and CM5, respectively, showing CNT emerging out of the surface fracture. $\mathrm{C} 10$ and $\mathrm{CM} 2$ show restricted grain-growth effect (and intergranular fracture). (f) Sample CM12 showing many CNT emerging out of large pores and straightened before breaking during the fracture [29]. 


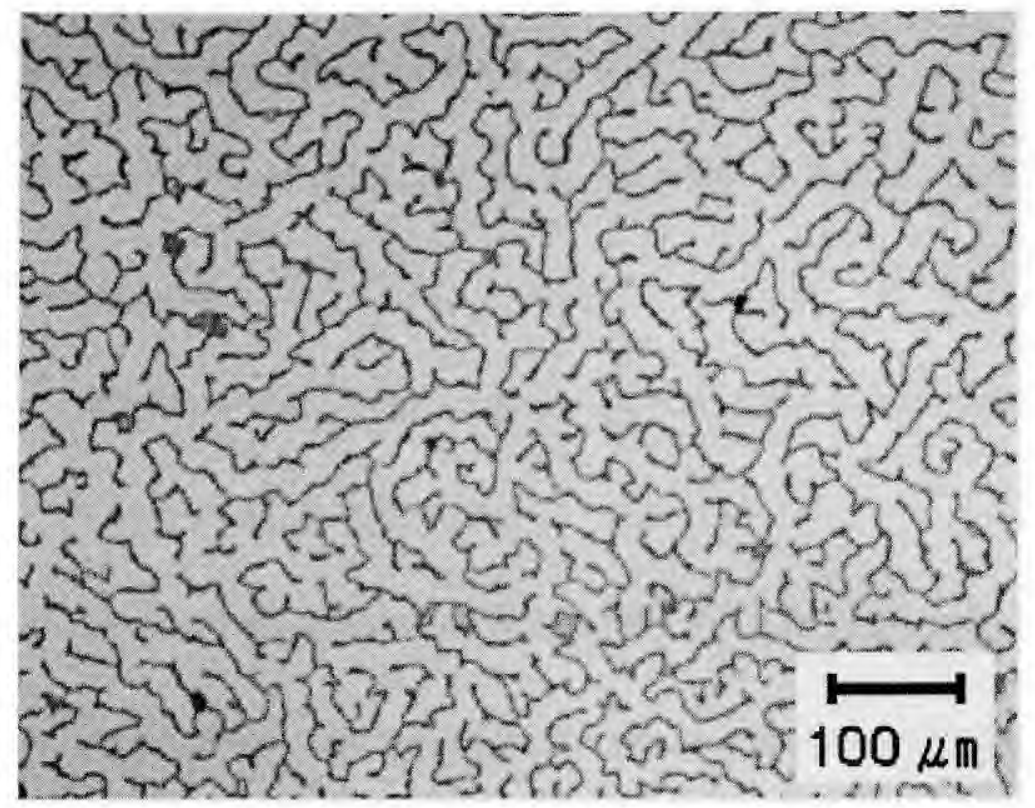

(a)

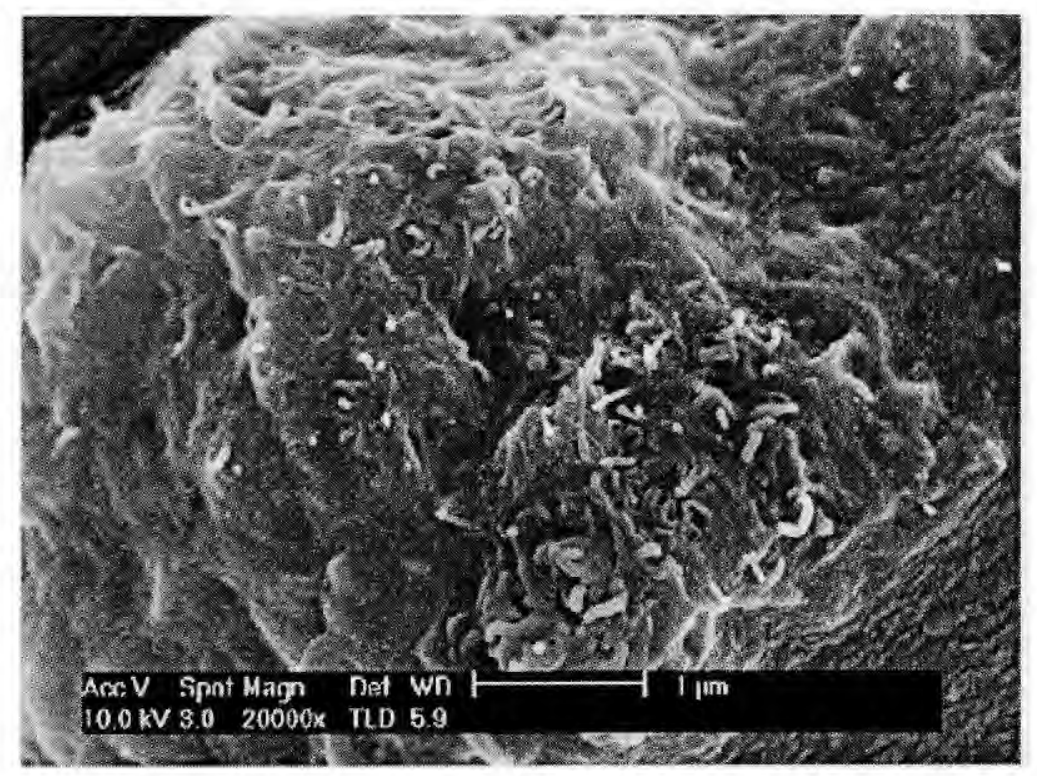

(b)

Fig. 2.28: The micrographs of carbon nanotube reinforced aluminum oxide composite powders. (a) The optical micrographs of dried gel and (b) the SEM micrographs of calcinated carbon nanotube/aluminum oxide composite powders [176]. 


\subsection{What's Missing and What's the Challenge?}

A review of the $\mathrm{Al}_{2} \mathrm{O}_{3}$-CNT nanocomposite in the previous section leads to following conclusions:

- Improvement in the fracture toughness has been achieved by addition of CNTs to the $\mathrm{Al}_{2} \mathrm{O}_{3}$ matrix.

- Uniform dispersion of CNTs in the $\mathrm{Al}_{2} \mathrm{O}_{3}$ matrix still remains a challenge.

- $\mathrm{Al}_{2} \mathrm{O}_{3}-\mathrm{CNT}$ nanocomposite processing/consolidation techniques are limited to solid state processing (e.g. HIP, sintering, spark plasma sintering, and extrusion).

- The interface between $\mathrm{Al}_{2} \mathrm{O}_{3} / \mathrm{CNT}$ is relatively less understood. $\mathrm{A}$ few works are limited to computational studies without sufficient experimental evidence.

Based on observations and limitations from other researchers' work, the present work addresses those specific challenges by synthesizing $\mathrm{Al}_{2} \mathrm{O}_{3}$-CNT nanocomposite coating by plasma spraying. In addition to the above listed challenges, this work also focus on the specific challenges which are unique to plasma spraying of $\mathrm{Al}_{2} \mathrm{O}_{3}-\mathrm{CNT}$ system. Some of these challenges are listed below:

- Improved CNT Dispersion: Achieving CNT dispersion in the powder makes powder treatment becomes a necessity in exercising them as required feedstock. 
- Dispersed CNTs allow interfacial interactions and load-transfers towards attaining best properties in ceramic nanocomposites. Consequently, layered deposition typical in plasma spraying will further improve CNT dispersion in the processed coating. Hence plasma spraying of pretreated (spray-dried) powder feedstock is incorporated in the current research to obtain coatings with improved fracture toughness.

- Bimodal Matrix Structure: Presence of second phases act as strengthening medium, thereby bimodal phase/grain generation can further enhance the fracture toughness of the nanocomposite. Consequently, controlling the bimodal microstructure becomes an immense challenge to overcome. Bimodal grain/phase can be obtained by imminent control of plasma processing parameters to result surface melting followed by resolidification and consolidation of core by solid state sintering. Further, extreme temperature $(>10000 \mathrm{~K})$ and impact $(\sim 1-3 \mathrm{Mach}$ velocity) associated with the plasma spraying creates an additional challenge of retaining CNTs in the processed coating [1, 39-41].

- $\mathrm{Al}_{2} \mathrm{O}_{3} / \mathrm{CNT}$ interface: Most of the ceramic-CNT processes involve "solid state processing" wherein aluminum oxide and CNT are both in solid state. But plasma spraying involves alumina in molten state, so wetting between alumina and CNT becomes highly important. This very novel concept of wettability of $\mathrm{Al}_{2} \mathrm{O}_{3} / \mathrm{CNT}$ 
interface is to be conceptualized, but currently there is no work dictating the interfacial mechanism occurring at the $\mathrm{Al}_{2} \underline{\mathrm{O}}_{3}-\mathrm{CNT}$ interface. And limited research on the aluminum oxide-graphite interface elicits non-wetting of the interface [90, 192-197]. Hence aspects like wettability, interfacial bonding, load transfer mechanism, etc. remain unanswered. Herein, ab-initio computer modeling of $\mathrm{Al}_{2} \mathrm{O}_{3}-\mathrm{CNT}$ interface has been incorporated towards eliciting interactive response at the $\mathrm{Al}_{2} \mathrm{O}_{3} / \mathrm{CNT}$ interface.

Figure 2.29 summarizes the $\mathrm{Al}_{2} \mathrm{O}_{3} / \mathrm{CNT}$ nanocomposite research efforts by various groups, their conclusions and remaining challenges. 


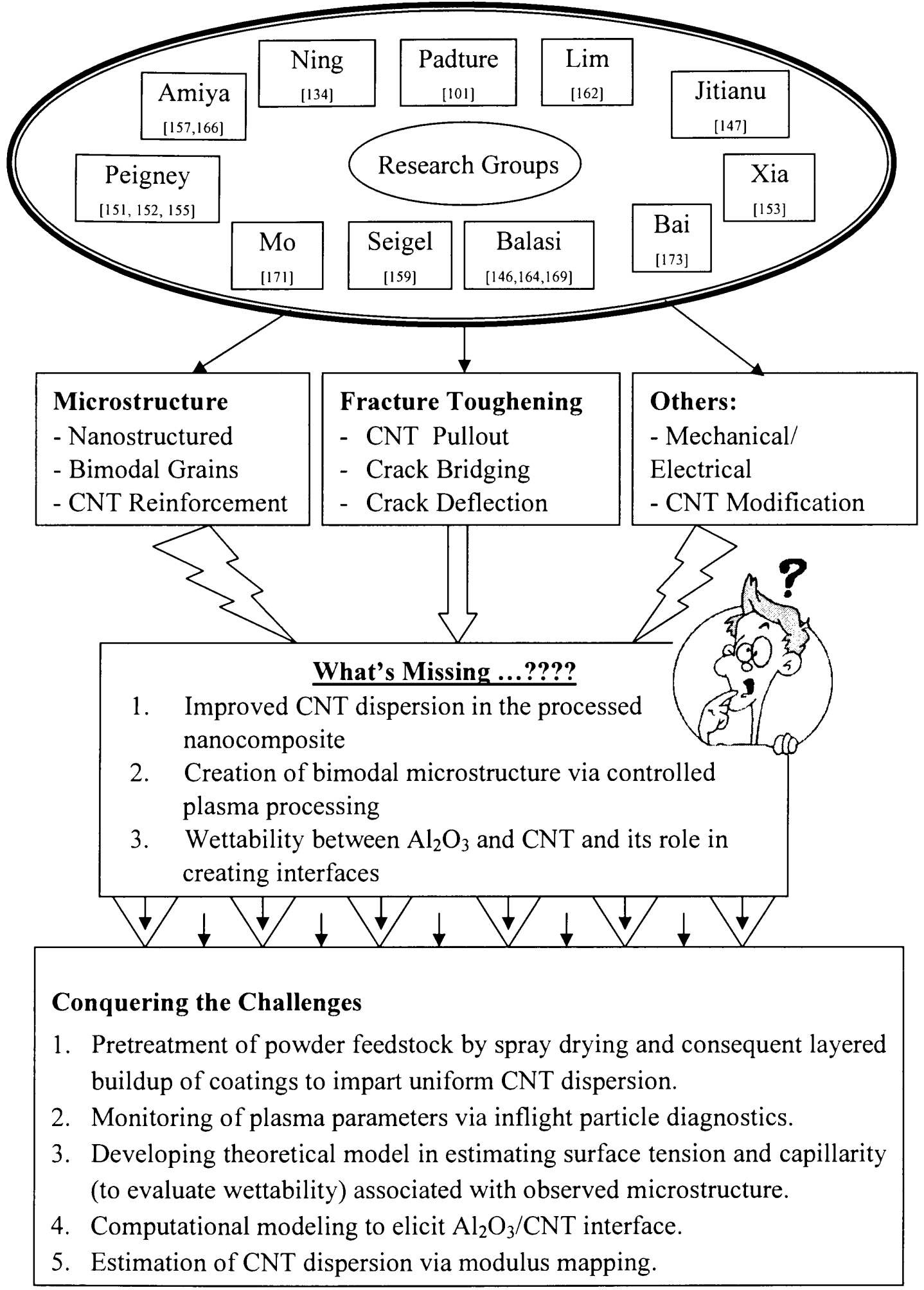

Fig. 2.29: $\mathrm{A}$ bird's view of $\mathrm{Al}_{2} \mathrm{O}_{3} / \mathrm{CNT}$ nanocomposite research efforts by various groups, their conclusions and remaining challenges. 


\section{EXPERIMENTAL PROCEDURE}

The aim of the current work is to fabricate $\mathrm{Al}_{2} \mathrm{O}_{3}-\mathrm{CNT}$ nanocomposite coating via plasma spraying with uniform dispersion of CNTs in the matrix for enhanced fracture toughness. Various methodologies of powder treatment are adopted for dispersion of CNTs in the powder feedstock.

\subsection{Pretreatment of Powder Feedstock}

Since nano size of the powders tend to clog the nozzle during plasma spraying, as-received nano $\mathrm{Al}_{2} \mathrm{O}_{3}$ and $\mathrm{CNTs}$ have been pretreated to result powder feedstock ideal for plasma spraying. Powder feedstock treatments prior to plasma spraying is explained in this section.

\subsubsection{As Received $\mathrm{Al}_{2} \underline{\mathrm{O}}_{3}$ Powder and CNTs}

Nano $\alpha-\mathrm{Al}_{2} \mathrm{O}_{3}$ powder $(99.8 \%$ pure, $150 \mathrm{~nm}$ particle size, and $40 \mathrm{~nm}$ crystallite size) was obtained from Inframat Corporation, Farmington, CT. Smooth and irregular faceted morphology of the as-received $\mathrm{Al}_{2} \mathrm{O}_{3}$ powder is presented in Fig. 3.1. Owing to increased surface area of the faceted nano particles, increased friction causes clogging of the nozzle during plasma spraying. Hence to reduce the clogging tendency of fine particles, powder treatment becomes necessary to reduce the contact area by consolidating them as spherical agglomerates.

Multiwall carbon nanotubes $(95 \%+$ purity, OD $40-70 \mathrm{~nm}, 0.5-2.0 \mu \mathrm{m}$ in length) were obtained from Nanostructured and Amorphous Materials Inc., Houston, TX. CNT 
diameter of about $70 \mathrm{~nm}$ is observed in the TEM image, Fig. 3.2, with a wall thickness of 25-30 $\mathrm{nm}$. In order to tap superior mechanical properties of CNTs, it becomes imperative to uniformly disperse CNTs in the matrix. But strong agglomerating tendency of CNTs require special treatments such as functionalization, molecular level dispersion, ultrasonication etc.

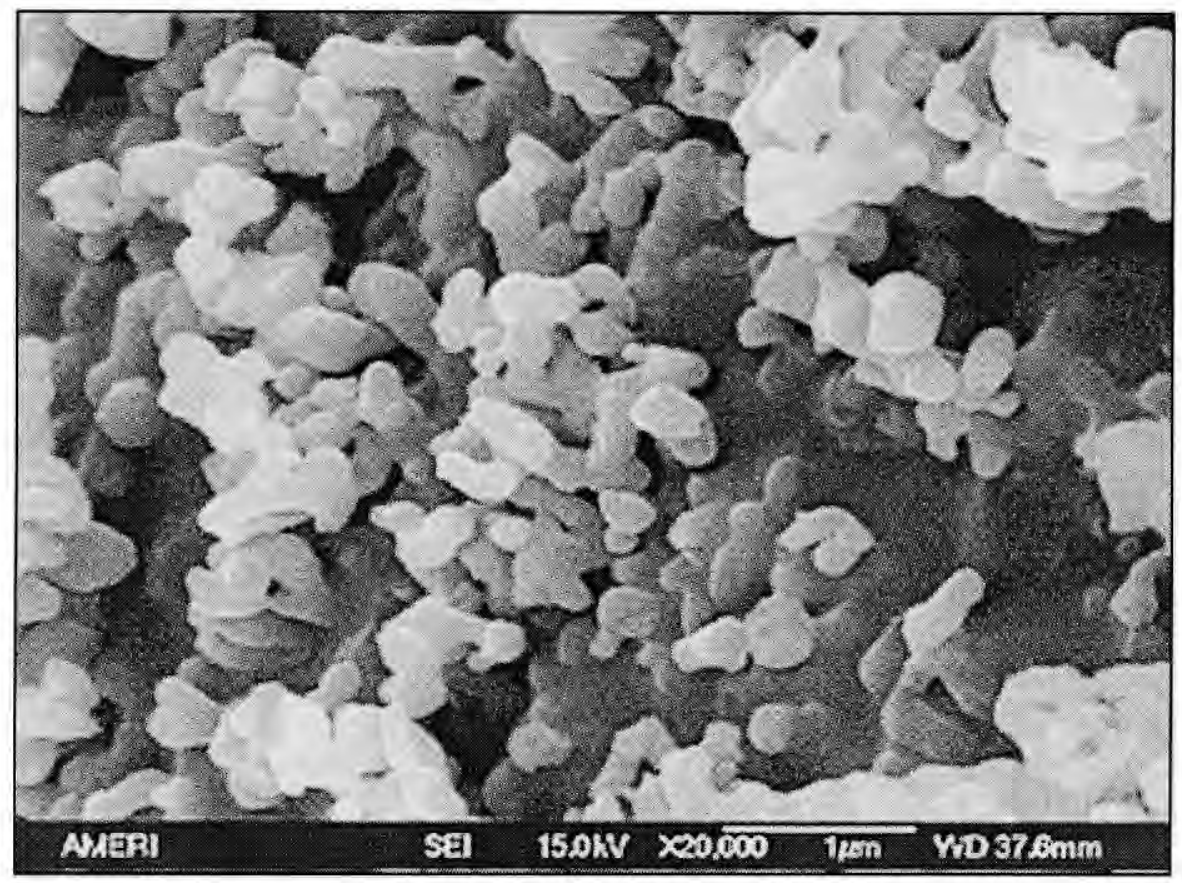

Fig. 3.1: As-received $\mathrm{Al}_{2} \mathrm{O}_{3}$ powder particles.

\subsubsection{Spray Drying of As-received $\mathrm{Al}_{2} \underline{\mathrm{O}}_{3}$ Powder (A-SD):}

Commercial spray drying process was utilized to obtain spherical agglomerates of $\mathrm{Al}_{2} \mathrm{O}_{3}$ particles (or mixture of $\mathrm{Al}_{2} \mathrm{O}_{3}$ and CNTs). Nanosize powder particles are dispersed in an aqueous organic binder to form slurry. The slurry is passed through an atomizing orifice, which mechanically binds the fine particles as spherical agglomerates. Resulting spherical agglomerates cause reduced interparticle friction, improved 
flowability and avoid clogging of powders in the nozzle during plasma spraying. Sieved spherical agglomerates in the size range of $15-60 \mu \mathrm{m}$ were obtained that are ideal for plasma spraying. Three powder treatment methodologies have been adopted to prepare the powder feedstock for consequent plasma spraying.

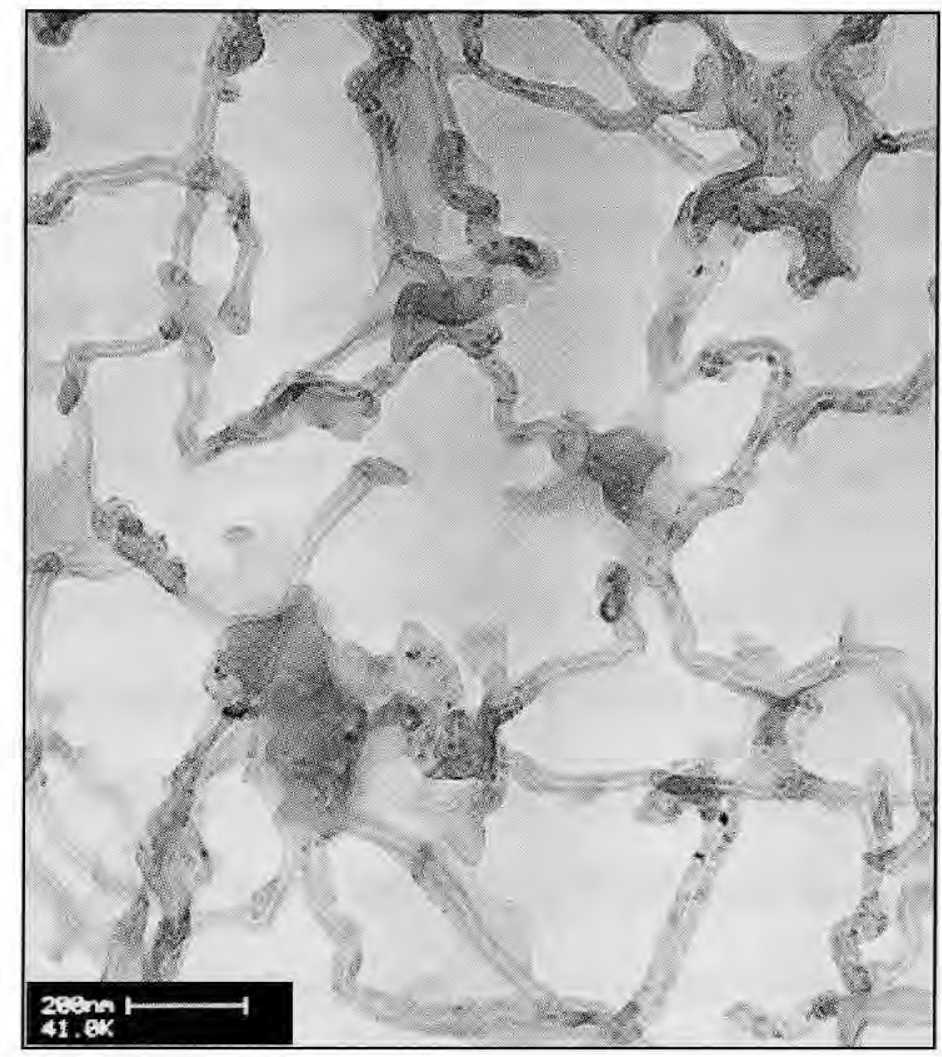

Fig. 3.2: TEM of Multiwalled CNTs.

Pure $\alpha-\mathrm{Al}_{2} \mathrm{O}_{3}(99.8 \%)$ was spray dried to obtain spherical agglomerates for powder feedstock (referred to as A-SD). Fig. 3.3a shows the typical size range of 15-60 $\mu \mathrm{m}$ spherical agglomerates, and an enlarged image, Fig. $3.3 \mathrm{~b}$ elicits the mechanically bounded porous cake of fine powders. 


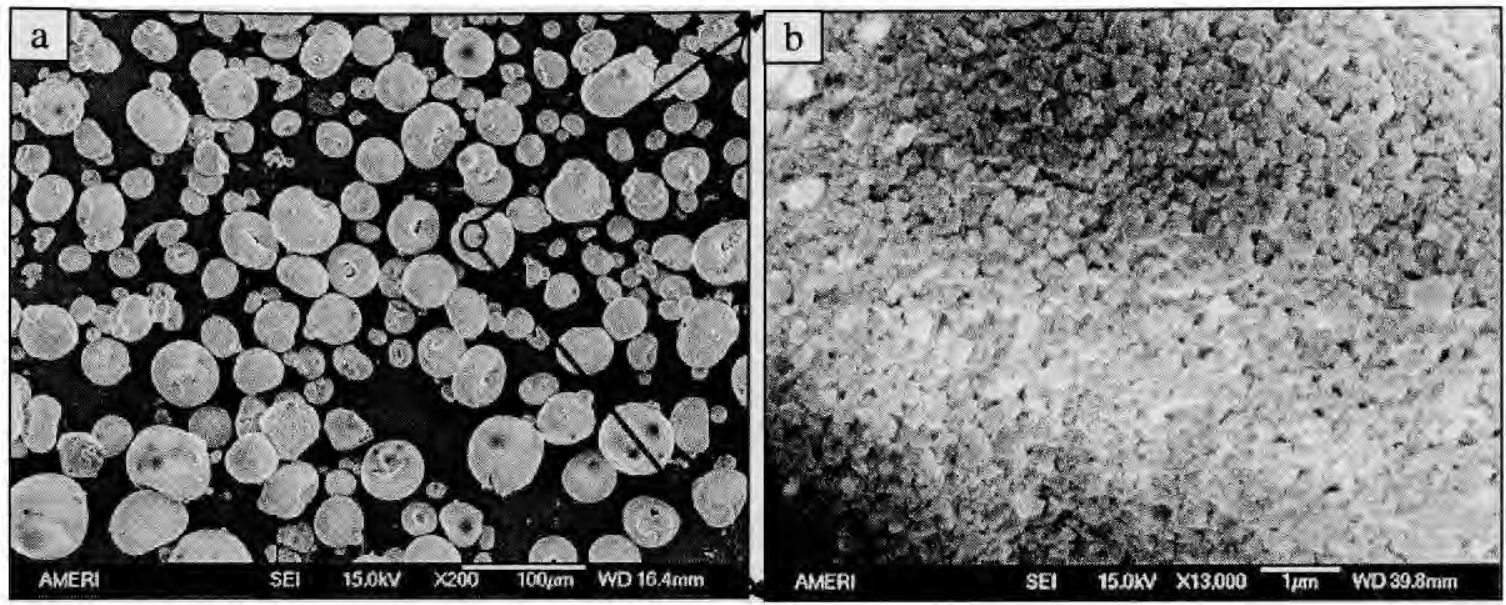

Fig. 3.3: a) Spray Dried $\mathrm{Al}_{2} \mathrm{O}_{3}$ powder, and b) Enlarged SEM image showing spraydried agglomerated mass of fine powders.

\subsubsection{Blending of Spray Dried $\mathrm{Al}_{2} \mathrm{O}_{3}$ with 4 wt. $\%$ CNT (A4C-B):}

Spray dried $\mathrm{Al}_{2} \mathrm{O}_{3}$ agglomerates were blended with $4 \mathrm{wt} . \% \mathrm{CNT}$ in a jar mill (US Stoneware, East Palestine, $\mathrm{OH}$ ) for $24 \mathrm{hrs}$ to obtain dispersion of CNTs in $\mathrm{Al}_{2} \mathrm{O}_{3}$ matrix (referred to as A4C-B powder). Schematic and SEM morphology of as-received $\mathrm{Al}_{2} \mathrm{O}_{3}$, spray-dried $\mathrm{Al}_{2} \mathrm{O}_{3}$ agglomerate and resulting blended $\mathrm{AC}-\mathrm{B}$ powder has been shown in Fig, 3.4. CNTs are observed to disperse onto (i) surface of agglomerate spray dried $\mathrm{Al}_{2} \mathrm{O}_{3}$ powder and (ii) dome-cavity regions of spray dried powder.

\subsubsection{Spray Drying of Composite $\mathrm{Al}_{2} \underline{\mathrm{O}}_{3}$ and CNT:}

Further refinement of CNT dispersion to what obtained in A4C-B powder can be achieved by spray drying of composite $\mathrm{Al}_{2} \mathrm{O}_{3}$ and CNT powder in a single step. Two powder compositions, namely $\mathrm{Al}_{2} \mathrm{O}_{3}$ - with 4 wt. \% CNT and 8 wt. \% CNT were used for composite spray drying: 
Nano $\mathrm{Al}_{2} \mathrm{O}_{3}$
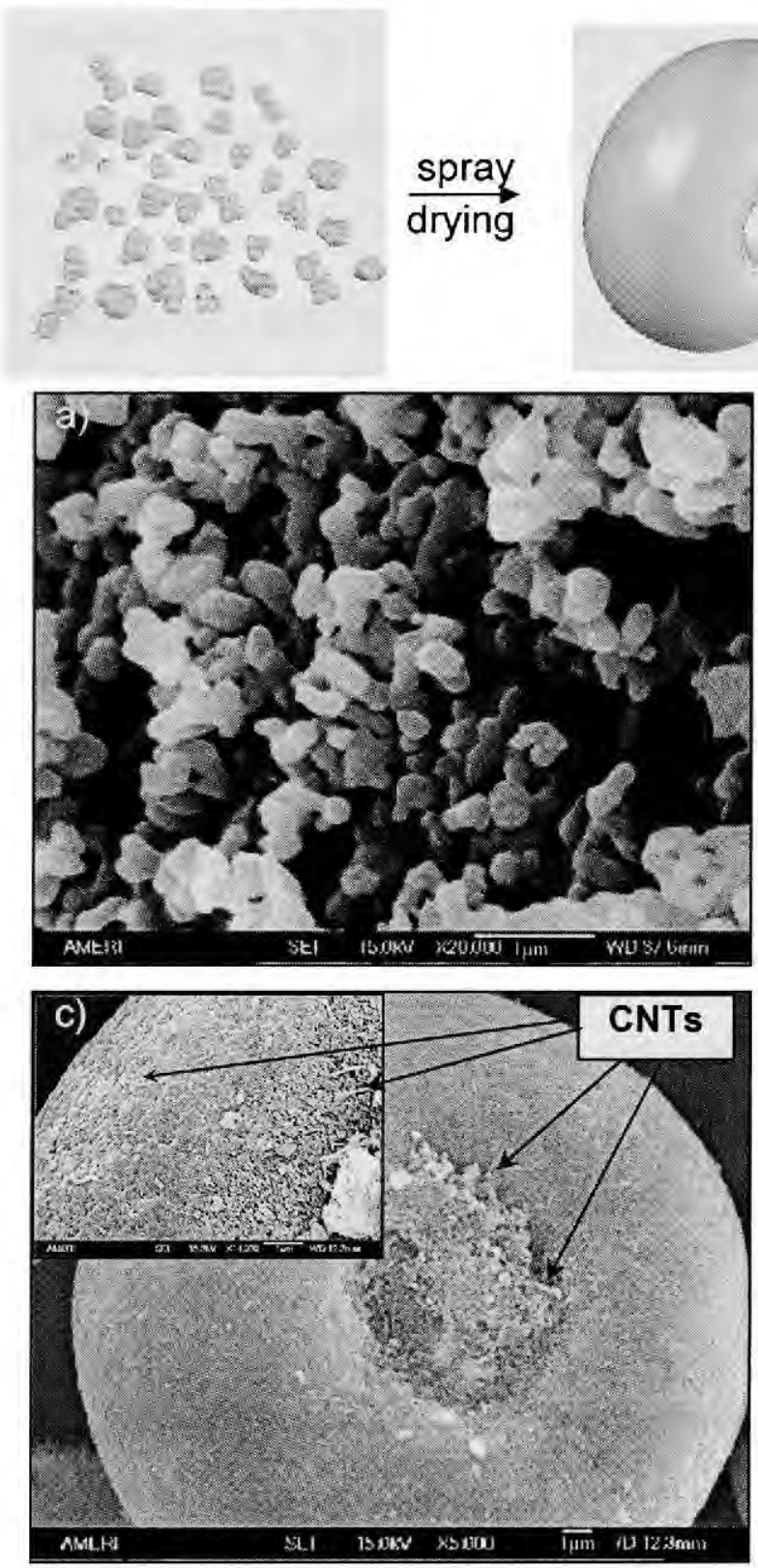

Spray Dried $\mathrm{Al}_{2} \mathrm{O}_{3}$
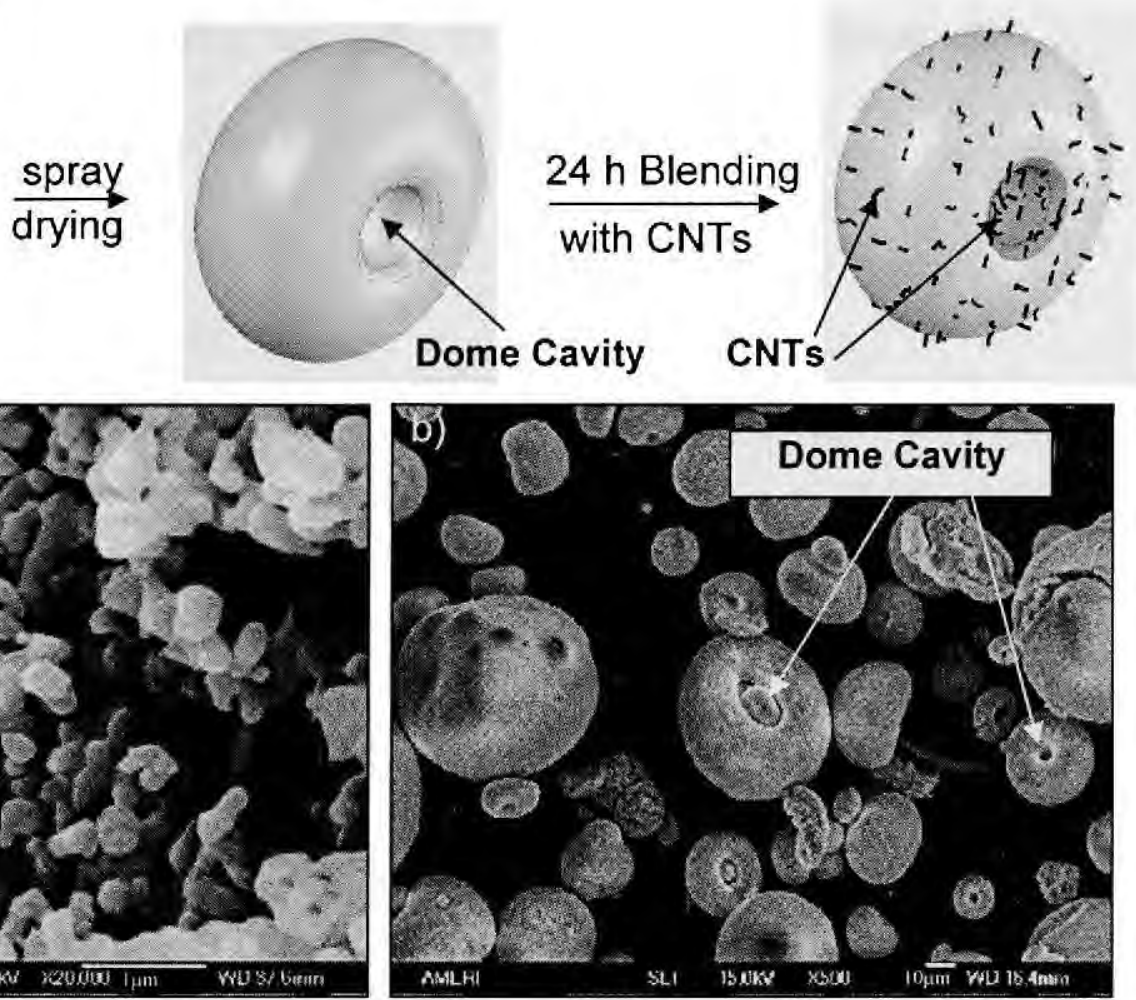

Fig. 3.4: Process schematic and SEM images of a) nano $\mathrm{Al}_{2} \mathrm{O}_{3}$, b) spray dried $\mathrm{Al}_{2} \mathrm{O}_{3}(\mathrm{~A}-\mathrm{SD})$ agglomerates, and c) spray dried $\mathrm{Al}_{2} \mathrm{O}_{3}$ blended with 4 wt. \% CNT for $24 \mathrm{~h}$ to result nanocomposite $\mathrm{A} 4 \mathrm{C}-\mathrm{B}$ powder feedstock.

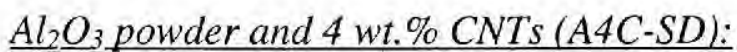

Spray drying of composite $\mathrm{Al}_{2} \mathrm{O}_{3}$ powder and $4 \mathrm{wt} . \% \mathrm{CNTs}$ (referred to as $\mathrm{A} 4 \mathrm{C}$ $S D$ ) produced spherical agglomerates of $15-60 \mathrm{Dm}$, Fig. 3.5a. In contrast to surface distribution of blended powder (Fig. 3.4), CNTs are also dispersed both at the powder 
agglomerate surface and the inside of the powder. No agglomeration of CNTs was observed along the powder surface, Fig. 3.5b. CNTs were also distributed uniformly inside the powder agglomerate, as seen in the fractured powder Fig. 3.5c.

\section{$\mathrm{Al}_{2} \underline{O}_{3}$ powder and $8 \mathrm{wt} \%$ CNTs $(A 8 C-S D)$ :}

Spray dried composite $\mathrm{Al}_{2} \mathrm{O}_{3}$ and 8 wt.\% CNTs $(A 8 C-S D)$ powder agglomerates show spherical morphology with size range of 15-60 Im, Fig. 3.6. Alike A4C-SD powder, uniform CNT dispersion is achieved in the powder agglomerate. Increase in the CNT content of the spray dried powder (from $4 \mathrm{wt} . \%$ to $8 \mathrm{wt} . \%$ ) is an extra parameter to analyze effect of CNT content in improving the fracture toughness of the plasma sprayed structure (apart from its different dispersion powder treatment when compared to A4C-B powder). Fracture surface of A8C-SD powder, Fig. 3.7, illustrates the non-agglomeration tendency of the spray-dried powder inside the powder agglomerate. This becomes significant since CNTs are dispersed non-preferentially everywhere uniformly in the powder agglomerate.

\subsection{Plasma Spraying of $\mathrm{Al}_{2} \mathrm{O}_{3}$-CNT Nanocomposite Coating}

Atmospheric plasma spraying, Fig. 3.8, of various powders is done using SG $100^{\circledR}$ plasma gun (Praxair Surface Technologies, Indianapolis, IN). Overall features of plasma spraying are listed in Table 3.1. Plasma processing parameters were optimized in terms of coating microstructure (partially melted, fully melted, and porosity), CNT distribution and fracture toughness. A representative set of plasma processing parameters is listed in Table 3.2. Consequently, A-SD, A4C-B, A4C-SD, A8C-SD powders were 

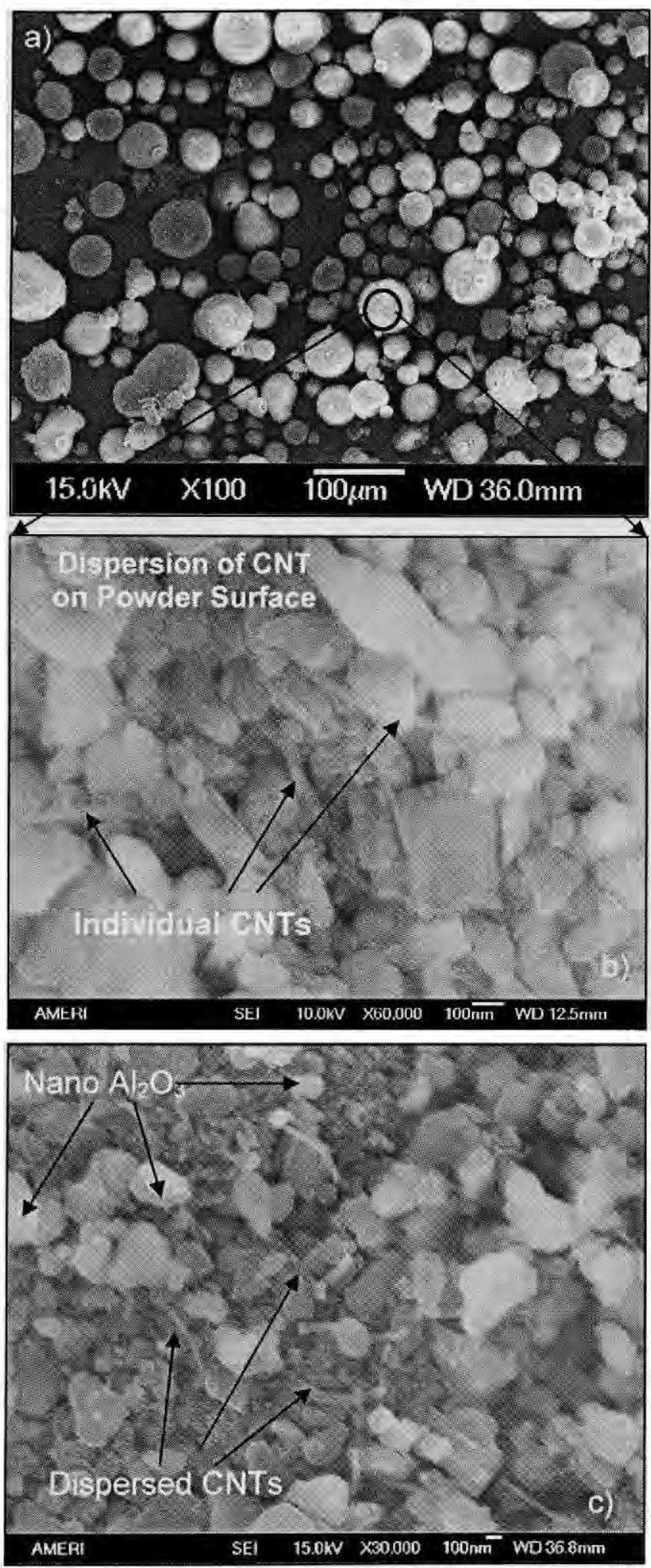

Fig. 3.5: SEM image of A4C-SD powder showing a) powder agglomerates, b) dispersion of individual CNTs, and c) fractured surface showing inside of the powder. 

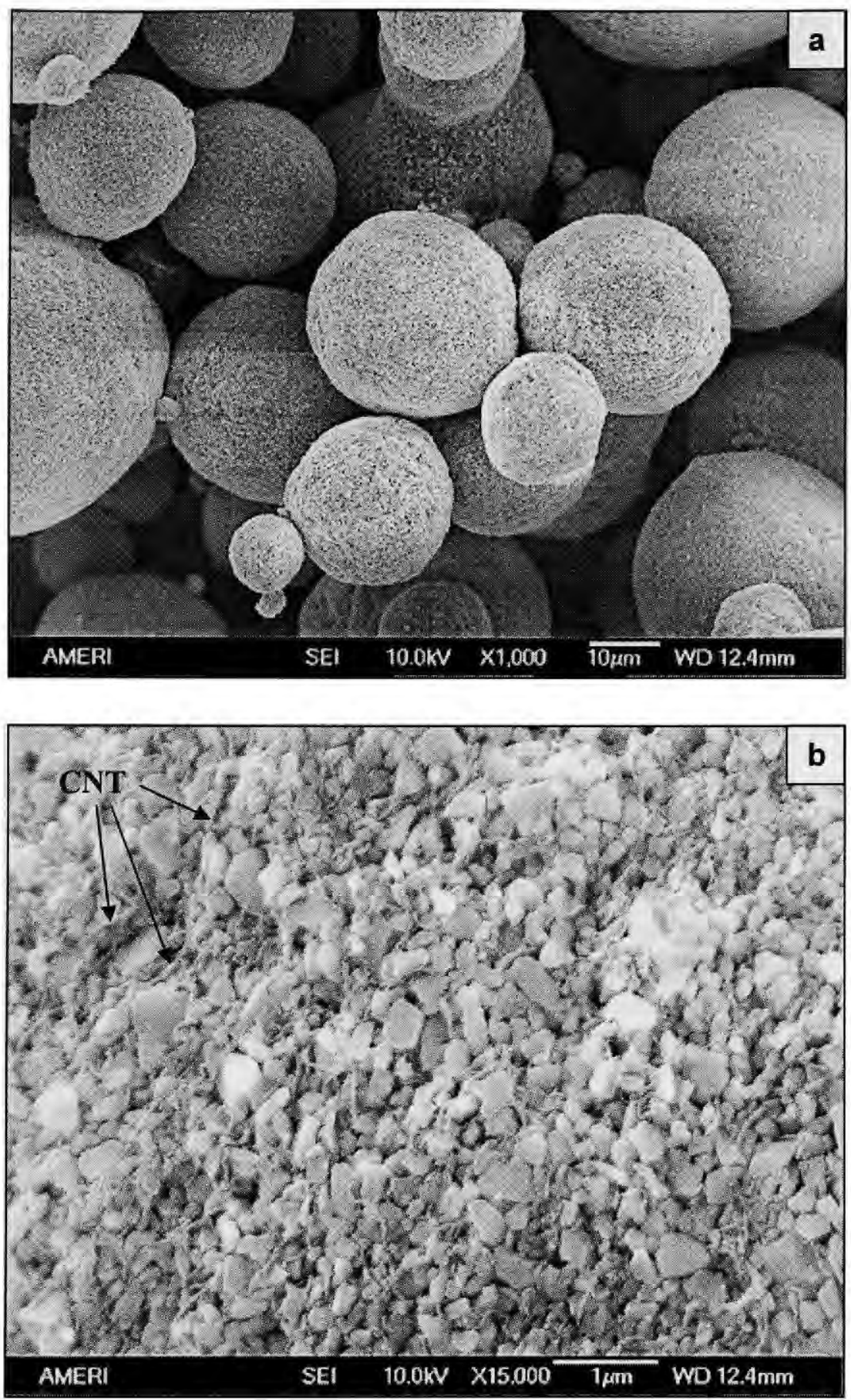

Fig. 3.6: Spray dried A8C-SD powder showing a) spherical agglomerates, and b) uniform CNT dispersion on surface. 


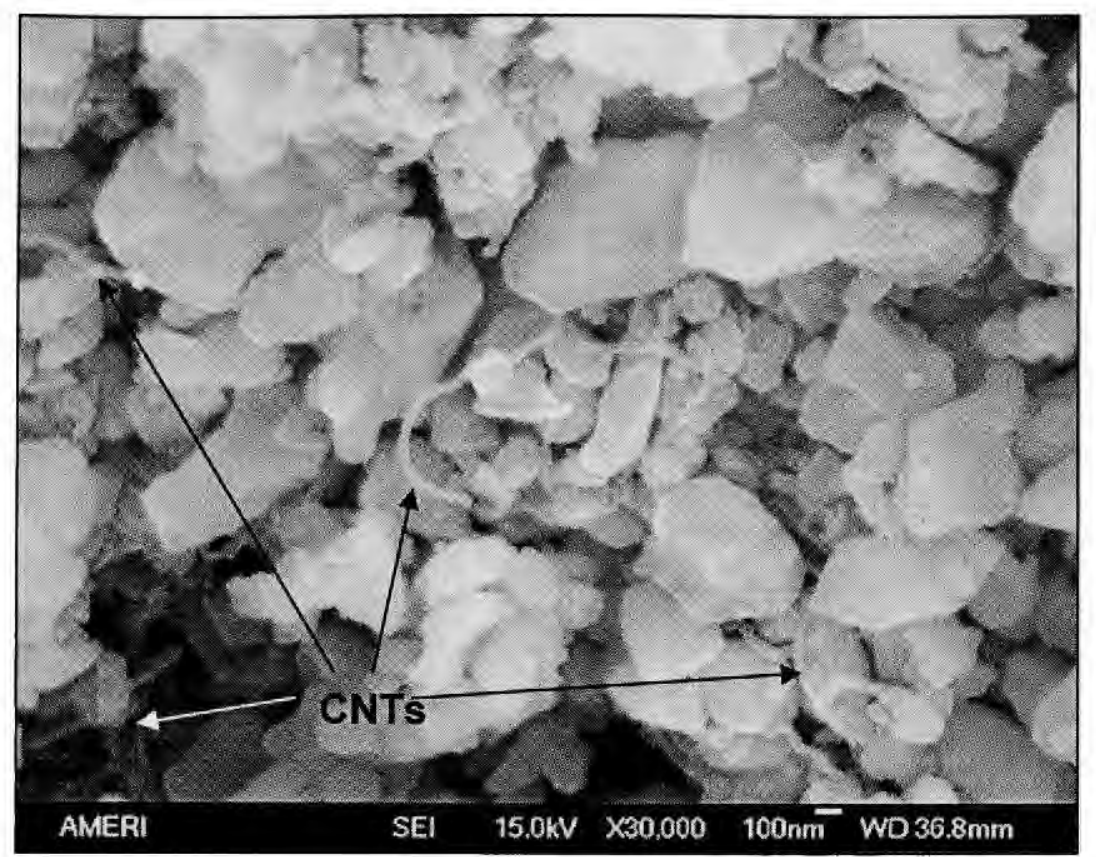

Fig. 3.7: Fracture surface of A8C-SD powder indicating uniform CNT dispersion in the core of the powder agglomerate.

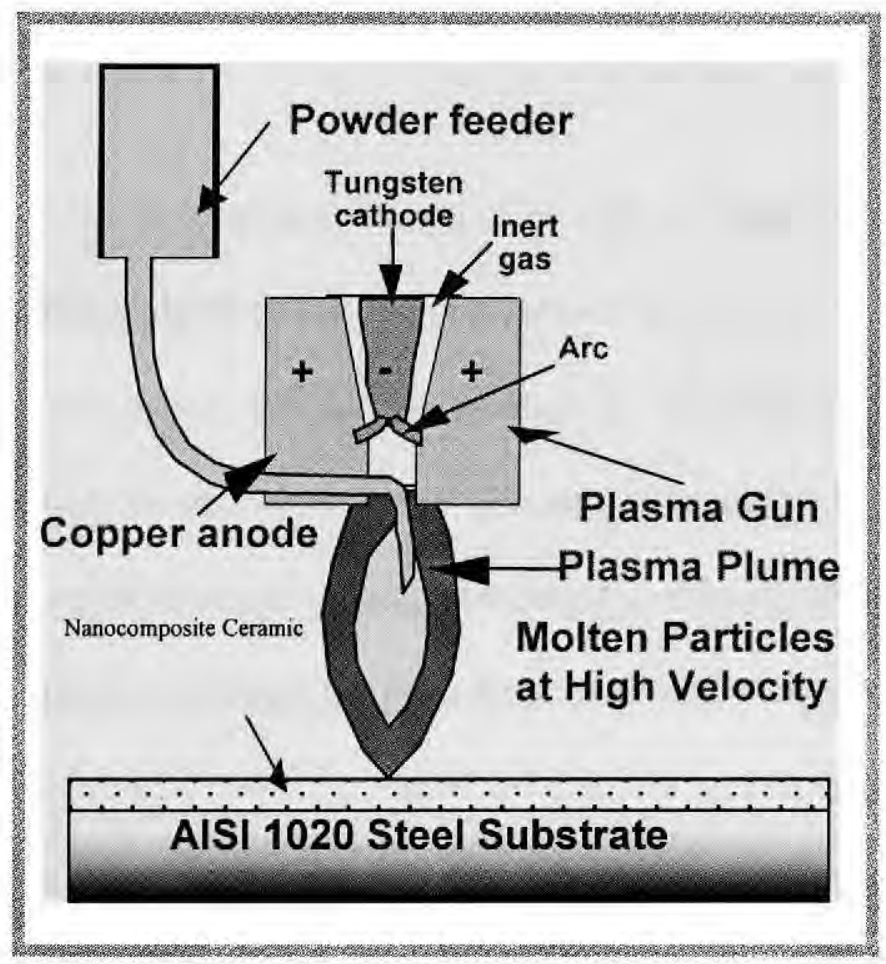

Fig. 3.8: Schematic of plasma spraying process. 
Table 3.1: Features of plasma spraying

\begin{tabular}{|c|c|}
\hline Features of Plasma Spraying & Description of the Feature \\
\hline Heat source & $\begin{array}{c}\text { Plasma arc with gun voltage and current 35 V } \\
\text { and 800 A, respectively }\end{array}$ \\
\hline Spray gun & $\begin{array}{c}\text { SG-100 } \\
\text { Technologies, Indianapolis, IN }\end{array}$ \\
\hline Particle velocity & $75-750 \mathrm{~m} / \mathrm{s}[198-201]$ \\
\hline Temperature & $10,000-15,000 \mathrm{~K}[198,200-202]$ \\
\hline Gases used & Primary gas: $\mathrm{Ar}$ \\
& $\begin{array}{c}\text { Secondary gas: } \mathrm{He} \\
\text { Carrier gas: } \mathrm{Ar}\end{array}$ \\
\hline
\end{tabular}

Table 3.2: Representative processing parameters for plasma spraying

\begin{tabular}{|c|c|c|c|c|c|c|c|}
\hline Plasma Spraying & $\begin{array}{c}\text { Volts } \\
(\mathbf{V})\end{array}$ & $\begin{array}{c}\text { Current } \\
(\mathbf{A m p})\end{array}$ & $\begin{array}{c}\text { Power } \\
(\mathbf{k W})\end{array}$ & $\begin{array}{c}\text { Feed } \\
\text { Rate } \\
(\mathbf{r p m})\end{array}$ & $\begin{array}{c}\text { Primary } \\
\mathbf{G a s} \\
\left(\mathbf{s l m}^{*}\right)\end{array}$ & $\begin{array}{c}\text { Secondary } \\
\left.\mathbf{G a s}_{(\mathbf{s}} \mathbf{s l m}^{*}\right)\end{array}$ & $\begin{array}{c}\text { Carrier } \\
\mathbf{G a s} \\
\left(\mathbf{s l m}^{*}\right)\end{array}$ \\
\hline $\begin{array}{c}\mathrm{Al}_{2} \mathrm{O}_{3} \text { with } \mathrm{CNT} \\
\text { coatings }\end{array}$ & $35-40$ & $600-780$ & $\begin{array}{c}18.5- \\
32.3\end{array}$ & $\begin{array}{c}2.5- \\
3.0\end{array}$ & $\begin{array}{c}\mathrm{Ar} \\
(32.1)\end{array}$ & $\mathrm{He}(59.5)$ & $\begin{array}{c}\mathrm{Ar} \\
(19.8- \\
21.7)\end{array}$ \\
\hline
\end{tabular}

*slm: standard liters per minute

plasma sprayed onto AISI 1020 steel substrate (100 x $20 \times 3 \mathrm{~mm}^{3}$ coupon) for detailed evaluation. A typical plasma sprayed coating is presented in Fig. 3.9.

Control of plasma parameters can be attained by inflight monitoring of powder particles in plasma plume using AccuraSpray (Tecnar Automation Ltée, QC, Canada), Fig. 3.10. This allows extra leverage in understanding the relation of plasma parameters responsible for generating resulting coating and microstructure. Optical fiber measures the particle velocity and temperature by controlled distance traveled by particle and the differential wavelength emission respectively. Particle velocity is indicative of degree of flattening of particles whereas particle temperature suggests its heated/melted state. 
Degree of melting and flattening of particles directly constitute the microstructure of the coating that affects its properties.

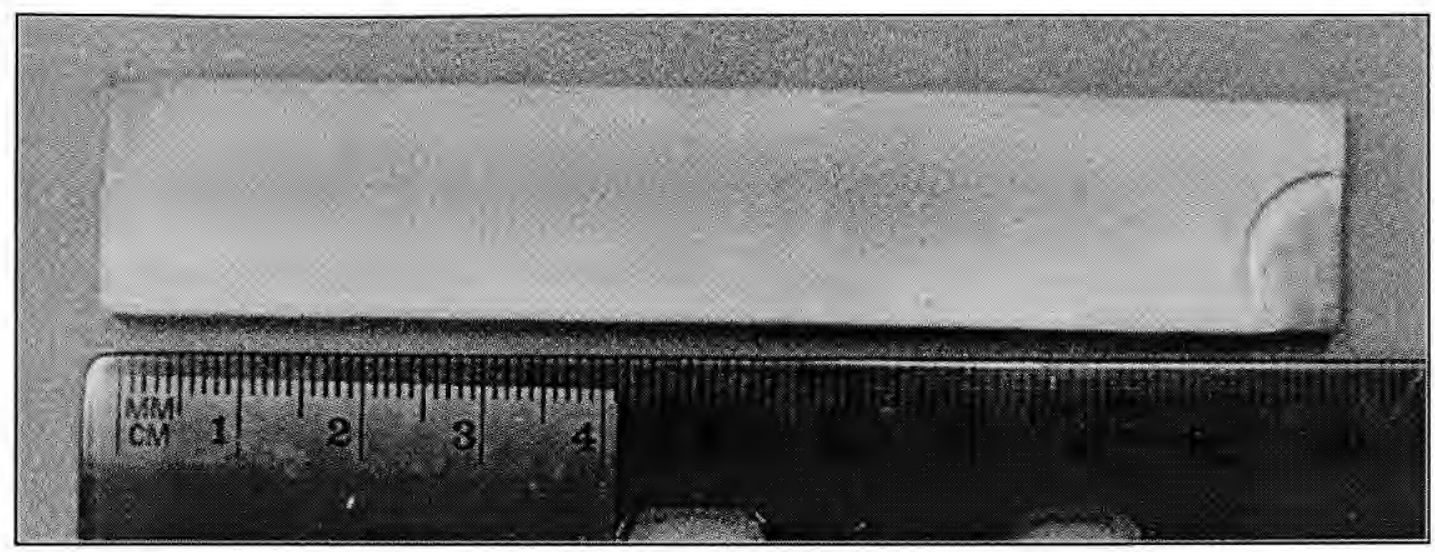

Fig. 3.9: Typical plasma sprayed coating showing uniform coating thickness.

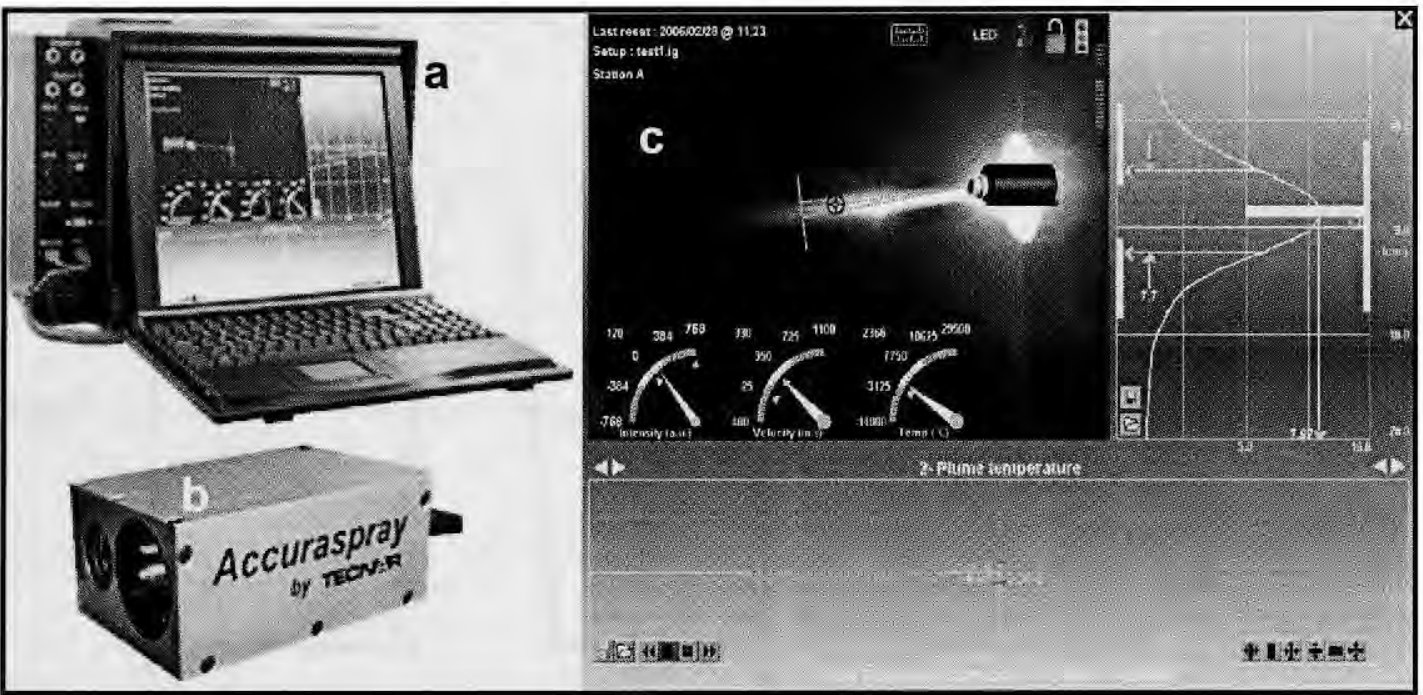

Fig. 3.10: AccuraSpray: Inflight particle diagnostic sensor: (a) Controller with display monitor, (b) the sensor head, and (c) a screenshot showing temperature, velocity and plume profile. 
The particle velocity is measured based on a time of flight technique. The sensor head images two optical fibers in space. As the particles pass through these images incandescent light is gathered and transmitted to photo multiplier tubes that generate an electronic pulse that corresponds to the particle passing through the fiber images, Fig. 3.11. The figure below shows a representation of the two pulses generated as the particle passes in front of the first and second fiber. A trigger level is set on the slope of each pulse to start and stop a timing clock. The velocity, $v$, is given by,

$$
v=\frac{D}{t_{2}-t_{1}}
$$

where $\mathrm{D}$ is the physical spacing of the two fibers imaged at the measurement volume and $t_{2}-t_{l}$ is the measured time it takes for the particle to fly past the two fibers.

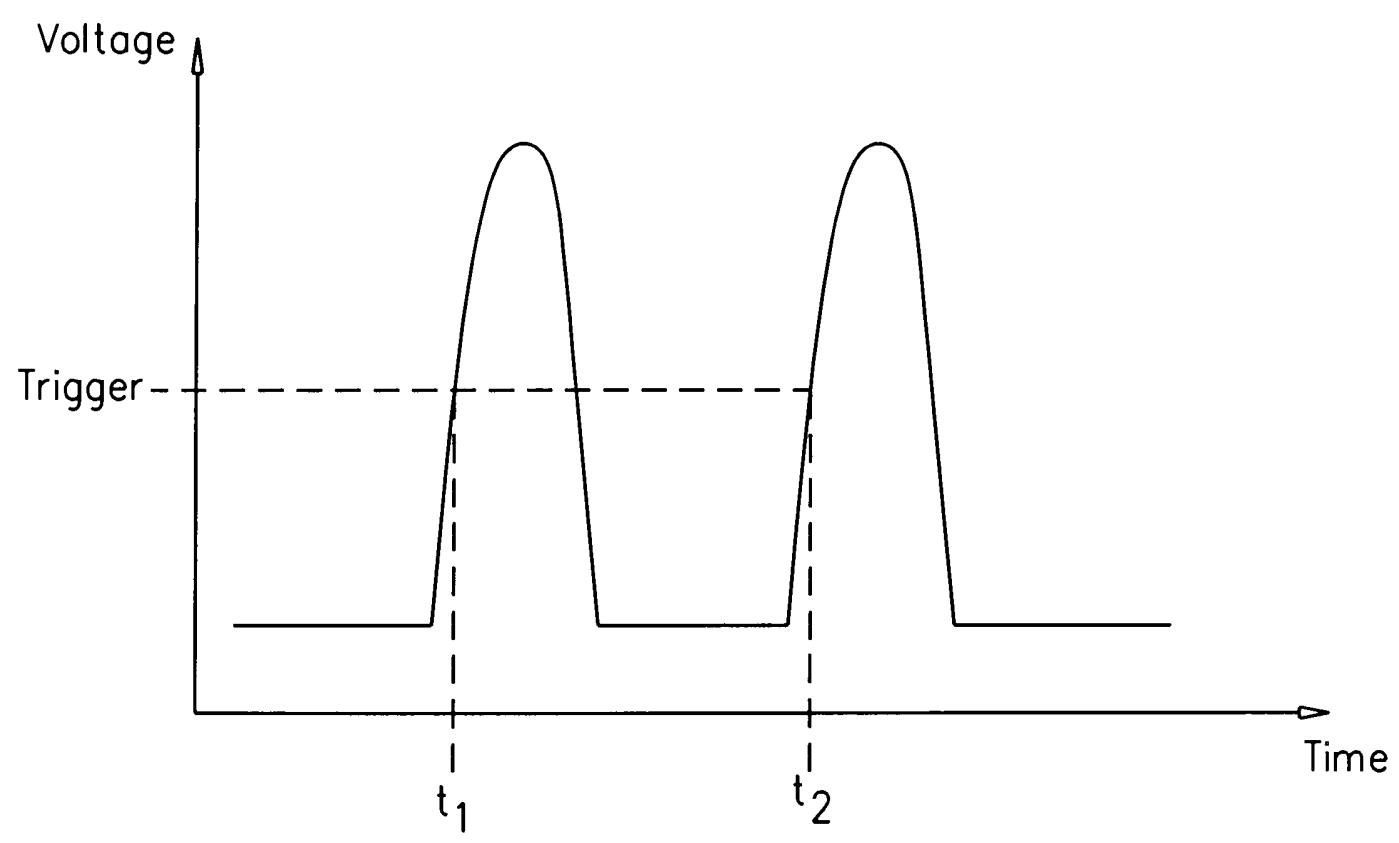

Fig. 3.11: Detection of particle velocity from the time traveled within fixed distance between two optical fibers. 
The basic premise behind all radiation thermometry is Plank's Law, which describes the emissive power of a radiating body as a function of wavelength, emissivity and temperature. Dual-wavelength (ratio or two color) pyrometry involves the measurement of the spectral energy in two different wavelength bands. Using Wien's approximation to Planck's law the ratio of radiant energy, $\mathrm{R}$, in two different wavelength bands, $\lambda_{1}$ and $\lambda_{2}$, is given by:

$$
\frac{I_{\lambda_{1}}}{I_{\lambda_{2}}}=R=\frac{\varepsilon_{\left(\lambda_{1}, T\right)}}{\varepsilon_{\left(\lambda_{2}, T\right)}}\left(\frac{\lambda_{1}}{\lambda_{2}}\right)^{-5} \exp \left[-\frac{C_{2}}{T}\left(\frac{1}{\lambda_{1}} \cdot \frac{1}{\lambda_{2}}\right)\right] \quad \text { Equation } 3.2
$$

where $T$ is the surface temperature of the radiating body, $C_{2}$ is a constant $(=1.4388$ $\mathrm{cm} . \mathrm{K})$, and $\varepsilon_{(\lambda, \mathrm{T})}$ is the spectral emissivity. AccuraSpray utilizes a CCD camera in capturing the plume intensity of plasma plume.

And solving for T gives:

$$
\frac{1}{T}=\frac{\ln \left[\frac{\varepsilon_{\left(\lambda_{1}, T\right)}}{\varepsilon_{\left(\lambda_{2}, T\right)}}\left(\frac{\lambda_{2}}{\lambda_{1}}\right)^{5} \frac{I_{\left(\lambda_{2}\right)}}{I_{\left(\lambda_{1}\right)}}\right]}{c_{2}\left(\frac{1}{\lambda_{1}}-\frac{1}{\lambda_{2}}\right)}
$$

Temperature and velocity of the powder particles is major factor in deciding the development of microstructure in a coating. Density, degree of melting, cooling rate, degree of impact, etc are deciding factors in deposition of a coating. Hence access to the inflight particle diagnostic data is beneficial in relating the microstructure in terms of experienced thermal history. 


\subsection{Microstructural and Phase Characterization}

\subsubsection{Optical Microscopy and Quantitative Analysis of CNT Dispersion}

Quantitative and morphological analysis of plasma sprayed coatings is performed using an optical microscope (Versamet 3, Buehler Ltd, IL, USA) with an attached CCD camera (Leica DFC 320). CNT dispersion in the powder feedstock was measured using Image-Pro ${ }^{\circledR}$ Plus, Version 5.1 imaging software (2004 Media Cybernetics Inc.) on SEM images. Development of microstructures in terms of fully melted, partially melted and porosity in the plasma sprayed coatings were quantified using imaging software. Partially melted region is recognized with solid state sintering of the particles showing necking/sintering at the particle interface. Fully melted region is characterized by melting and resolidification of the powder particles. Porosity is the region of voids (inter-splat, intra-splat or unfilled regions). Quantification of such features helps in assimilating the role of regions in enhancing the fracture toughness and optimizing the plasma processing parameters.

SEM images were used in analyzing CNT dispersion through Image-Pro imaging software. Quantitative image analysis illuminates the nature of CNT dispersion in the various treated powders. Angular alignment of CNTs in the dome cavity or surfacedispersion of CNTs in the powder agglomerate allows significant inference to the powder behavior during plasma spraying and consequent coating microstructure. Moreover, uniform CNT dispersion also becomes obvious through the quantitative analysis depicting uniform spreading of CNTs homogeneously in the matrix. 


\subsubsection{Phase Analysis in Powder Feedstock and Plasma Sprayed Coatings}

Phase analysis of the powders and coatings was carried out using a Siemens 500D X-ray Diffractometer (XRD) with $\mathrm{CuK}_{\alpha}$ radiation (of $1.54 \AA$ ) operating at $40 \mathrm{kV}$ and 20 mA. A graphic software DIFFRAC ${ }^{\text {plus }}$ EVA (Version Rev 0, Bruker axs, Madison, WI, USA) was used to analyze the XRD spectra. Crystallite size $(t)$ measurement was carried out using Scherrer equation, equation 3.4. Standard NIST Si powder sample was employed to determine the instrumental broadening and the calibration of the XRD patterns of powder samples using Gaussian profile.

$$
t=\frac{0.9 \lambda}{B \cos \theta} \quad \text { Equation } 3.4
$$

where $\lambda$ is the incident wavelength, and $\theta$ is the Bragg angle, and $B=\sqrt{B_{m}^{2}-B_{s}^{2}}$, where $B_{m}$ and $B_{s}$ are full width half maximum (FWHM) broadening of specimen and standard sample respectively.

\subsubsection{Confirmation of CNT Retention by Raman Spectroscopy}

Micro-Raman spectroscopy of the powder feedstock and plasma sprayed nanocomposites was carried out to validate the carbon nanotube structure. Ti-sapphire crystal target with a laser wavelength of $785 \mathrm{~nm}$ was used for this purpose. The laser was produced using a laser source from Spectra Physics (Model 3900S, California, USA) and the detector was from Kaiser Optical Systems, Inc. (Michigan, USA). 


\subsubsection{Microstructural Characterization of $\mathrm{Al}_{2} \underline{O}_{3}-\mathrm{CNT}$ Nanocomposite}

Coating morphology and CNT dispersion in the powders and plasma sprayed deposits was investigated using FESEM JEOL JSM $6330 \mathrm{~F}$ scanning electron microscope. Samples were metallographically polished to $0.5 \mu \mathrm{m}$ using diamond slurry for preparing sample surface. No sample preparation was done for imaging powders and fractured coating surfaces. These were consequently gold coated (using Pelco SC-7 Auto Gold Sputter coater) for 30 seconds to allow earthing of electronic charge. SEM was operated at $15 \mathrm{kV}$ and $12 \mu \mathrm{A}$ for microstructure observation. Elemental analysis of the composite was determined by performing energy dispersive spectroscopy (EDS) integrated with the FESEM.

Transmission electron microscope (TEM) was used to analyze the CNT distribution and observe $\mathrm{Al}_{2} \mathrm{O}_{3}$-CNT interface in the plasma sprayed coatings. Two different types of TEMs were used in characterizing the coatings, viz. (i) Philips PW 6061 TEM system (model CM 200, Eindhoven, Netherlands), and (ii) FEI Technai F30 high-resolution transmission electron microscope operated at $300 \mathrm{kV}$. Samples for TEM observation were fabricated by two methods: (i) crushing and dispersion in ethanol followed by ultrasonification (Branson 2510, Danbury, CT), and (ii) metallographic thinning, dimpling (Gatan, Inc. Model $656 \mathrm{Mk3}$, California, USA) and ion milling (Gatan Precision Ion Polishing, Model 691, California, USA). 


\subsection{Mechanical Testing}

\subsubsection{Vicker Indentation Toughness}

Zhongguo HXD-100 TMC Shanghai Taiming Optical Instruments microhardness tester (200g load and $15 \mathrm{~s}$ dwell time) was used for estimating hardness and fracture toughness of the plasma sprayed samples using indentation technique. Student t-test was utilized, with better than $95 \%$ confidence level, towards comparing the statistical difference between the fracture toughness values for eight indentations on each sample. Fracture toughness was calculated from the Anti's semi-empirical relationship based on radial crack generation during Vicker indentation [203].

\subsubsection{Nanoindentation}

The elastic modulus values of the as-sprayed nanocomposites in modulus mapping mode were evaluated by the nanoindentation technique. Indentation experiments were conducted using Hysitron TriboIndenter ${ }^{\circledR}$ (Hystrion Inc., Minneapolis, MN). Three-sided Berkovich diamond indenter was used for indentation. The load and displacement data obtained from the tests were analyzed using the methods reported by Oliver and Pharr [204]. The initial calibration of the instrument was done using a standard aluminum and fused silica sample provided by Hysitron.

Modulus mapping is performed using dynamic mechanical analysis approach of applying cyclic stress in compression. Quasi-static load is applied to the probe tip with smaller dynamic load (at a predescribed frequency $(200 \mathrm{~Hz})$. Dynamic load is then analyzed to measure amplitude and phase shift of the original signal. The equation of motion for sinusoidal force $\left(F_{0}\right)$ is given as: 


$$
F_{0} \sin (\omega t)=m \ddot{x}+C \dot{x}+k x
$$

where $\mathrm{m}$ is the mass of sensor, $\mathrm{k}$ is the stiffness, and $\mathrm{C}$ is the damping of the system. And displacement response is given as:

$$
x=X \sin (\omega t-\phi)
$$

Equation 3.6

Solution to the differential equations are:

$$
X=\frac{F_{o}}{\sqrt{\left(k-m \omega^{2}\right)^{2}+(C \omega)^{2}}},
$$

and

$$
\phi=\tan ^{-1} \frac{\omega C}{k-m \omega^{2}}
$$

rearranging the equations, $\mathrm{k}$ and $\mathrm{C}$ can be calculates by:

$$
k=\frac{F_{0}}{X} \frac{1}{\sqrt{1+\tan ^{2} \phi}}+m \omega^{2}
$$

and

$$
C=\sqrt{\frac{\left(\frac{F_{0}}{X}\right)^{2} \tan ^{2} \phi}{1+\tan ^{2} \phi} \frac{1}{\omega}}
$$

During instrument set up, stiffness, damping and mass of sensor are evaluated by frequency sweep of sensor in air. Stiffness and damping of the instrument can be directly subtracted to obtain actual stiffness and damping of the tested sample. Correspondingly, storage modulus (E') and loss modulus (E") can be calculated as following:

$$
E^{\prime}=\frac{k_{s} \sqrt{\pi}}{2 \sqrt{A_{c}}}
$$




$$
E^{\prime \prime}=\frac{\omega C_{s} \sqrt{\pi}}{2 \sqrt{A_{c}}}
$$

where $A_{c}$ is contact area, which is dependent on the contact depth. Contact depth of the indenter is described through tip area function during instrument calibration.

\subsection{Ab-initio Molecular Modeling of $\mathrm{Al}_{2} \mathrm{O}_{3}$-CNT Interface}

SIESTA (Spanish Initiative for Electronic Simulations with Thousands of Atoms) 1.3 modeling scales $\mathrm{Al}_{2} \mathrm{O}_{3}$-CNT interface was created with $1 \times 1 \times 1$ crystal lattice of alpha-alumina interfacing $2 \times 2 \times 2$ crystal layers of graphite. Plane wave basis set was used for the interfacial system, limiting in $z$-direction and periodicity in $x-y$ direction with cell size of $4.928 \times 4.928 \times 26.4114 \AA^{3}$ and $\alpha=90^{\circ}, \beta=90^{\circ}$, and $\gamma=120^{\circ}$. Standard Kohn Sham self-consistent density functional was utilized with LCAO (Linear combination of atomic orbitals) basis set in local density approximations. Spin polarized Ceperly-Adler scheme (Perdew and Zunger) was used for defining $\mathrm{Al}, \mathrm{O}$ and $\mathrm{C}$ exchange correlation functional. Improved Troullier Martins pseudo potential generation was employed to describe nonlocal, and normconserving interaction between core and valence electrons. First principle pseudopotentials were generated from spin polarized non relativistic ground state components of Kleinman and Bylander projectors. $\mathrm{Al}$ was defined with ground state $3 \mathrm{~s}^{2} 3 \mathrm{p}^{1} 3 \mathrm{~d}^{0}$ with cutoff $1.86,2.25$ and 3.07 bohrs respectively [192]. Oxygen was stated as $2 \mathrm{~s}^{2} 2 \mathrm{p}^{4} 3 \mathrm{~d}^{0} 4 \mathrm{f}^{0}$ with cutoff $1.15,1.15,1.15$, and 1.15 bohrs respectively, whereas Carbon was described through $2 \mathrm{~s}^{2} 2 \mathrm{p}^{2}$ ground state with cutoff 1.50 and 1.54 bohrs respectively $[192,205]$. 
Localized spin density (LSD) Hamiltonian was calculated by matrix diagonalization to generate self-consistent Kohn Sham solution. Conjugate gradient (CG) method was used for coordinate optimization with limiting force of $0.05 \mathrm{eV} / \AA$ or 50 iterations whichever came first. Maximum displacement during CG optimization run was limited to 0.2 bohr. 


\section{RESULTS AND DISCUSSION}

Effect of CNT dispersion in enhancing the fracture toughness of $\mathrm{Al}_{2} \mathrm{O}_{3}-\mathrm{CNT}$ nanocomposite has been conferred to in this section. Role of powder pretreatment and optimized plasma spraying parameters in retaining and distributing CNTs are elucidated with underlying consolidation mechanism. Microstructural effect on mechanical property is undertaken to confirm the fracture toughness enhancement. Wettability studies and computational modeling is pursued for understanding the interfacial behavior between $\mathrm{Al}_{2} \mathrm{O}_{3}$ matrix and $\mathrm{CNT}$ reinforcement. Subsequent sections enunciate the overall behavior of plasma sprayed $\mathrm{Al}_{2} \mathrm{O}_{3}$-CNT nanocomposite interlinking the $\mathrm{CNT}$ dispersion with generated microstructure and toughening mechanisms.

\subsection{Powder Treatment of $\mathrm{n}-\mathrm{Al}_{2} \mathrm{O}_{3}$ as Ideal Feedstock for Plasma Spraying}

Nano- $\mathrm{Al}_{2} \mathrm{O}_{3}$ powder was spray dried to result A-SD powder (explained in section 3.1.2). Though the powder particles are agglomerated as spherical cake in the size range of $15-60 \mu \mathrm{m}$, there is no change in the phase of the treated powder particles, and no chemical reaction occurred during the spray drying. Identity of initial powder particles is maintained during the spray drying as confirmed by XRD spectrum, Fig. 4.1 and Raman Spectrum, Fig. 4.2. This powder (A-SD) is used as a feedstock for optimizing the plasma spraying parameters.

Spray dried aluminum oxide powder is referred to as A-SD from now onwards, and details of initial particle size, spray drying treatment, and adopted nomenclature are presented in Table 4.1. 


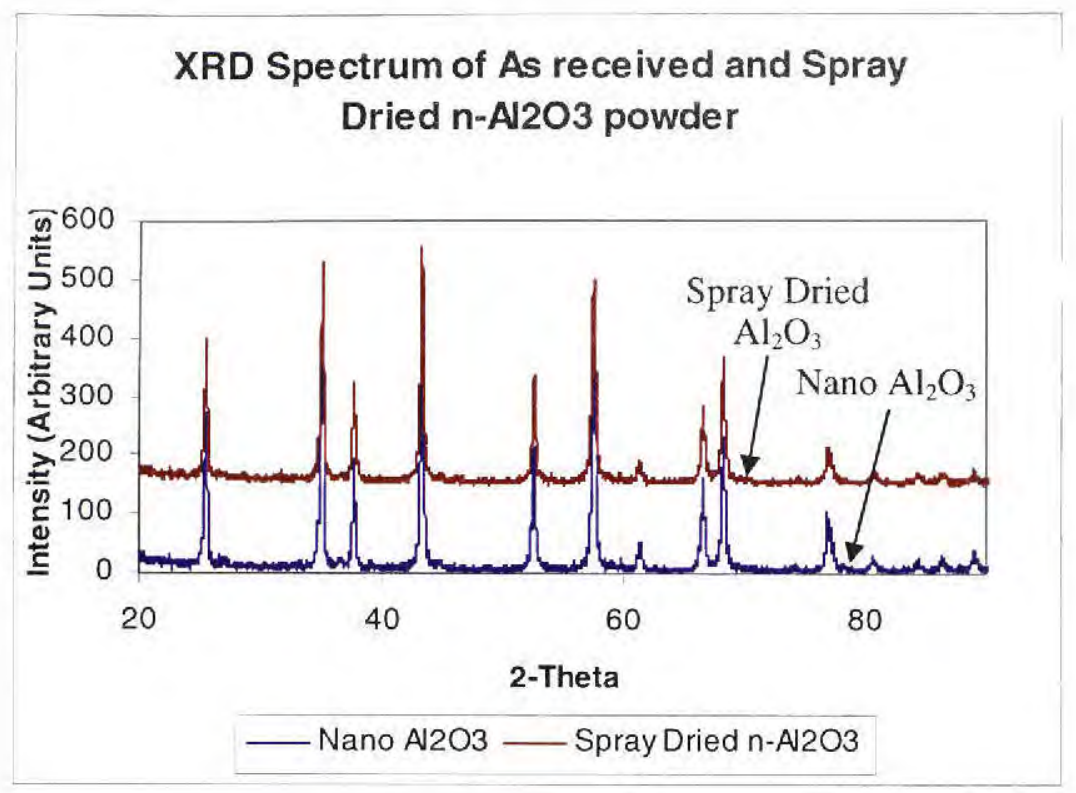

Fig. 4.1: $\mathrm{XRD}$ spectrum of as-received and spray dried $\mathrm{n}-\mathrm{Al}_{2} \mathrm{O}_{3}$ powder indicating no phase change and chemical reaction.

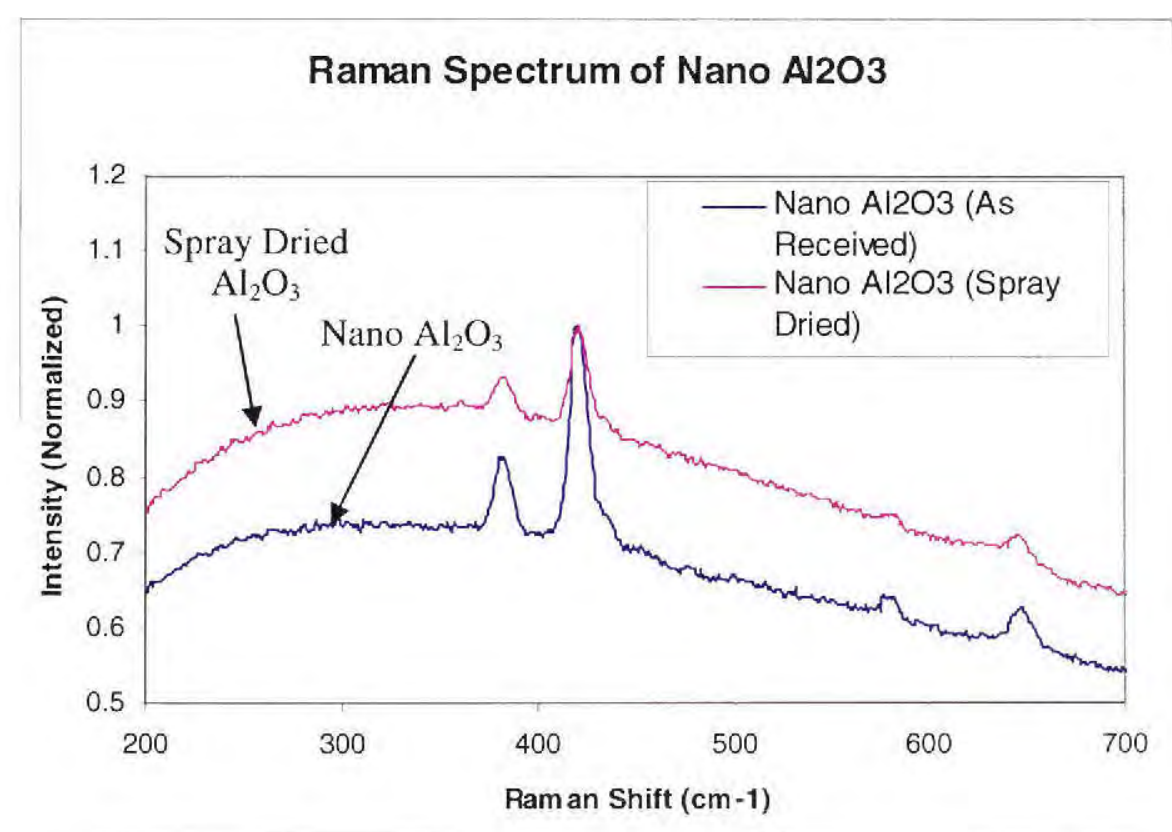

Fig. 4.2: Raman spectrum showing similar peaks in the as-received and spray dried n$\mathrm{Al}_{2} \mathrm{O}_{3}$ powder. 
Nano scale surfaces are known to be unstable because of their high surface to volume ratio, and have strong tendency towards agglomeration [206]. During mechanical blending of the A-SD and CNT powder mixture, agglomeration and settling of CNTs in the dome cavity of A-SD powder is observed in the scattergram, Fig. 4.3a, via Image Pro quantitative analysis. Agglomeration of CNTs in the histogram, Fig. 4.3b, endorses the higher CNT content in the dome cavity when compared to the CNTs adhering at the surface of the powder agglomerate. Though CNTs are dispersed in A4C-B powder to some extent, further improvisation of CNT dispersion can have tremendous impact on improving mechanical properties of the nanocomposite. This is achieved by composite spray drying of the $n-\mathrm{Al}_{2} \mathrm{O}_{3}$ and CNT. Laplace equation (of free energy change with particle radius) clearly elicits the instability of nanoparticles with the decreasing radius of particles. But, increasing the surface area by contacting two unstable surfaces reduces the overall energy of system to a lower value. CNTs, when mixed with nano- $\mathrm{Al}_{2} \mathrm{O}_{3}$ powder particles (during composite spray drying), reduce the surface charges by physically attaching to open surfaces[207]. Because of high surface area of nano- $\mathrm{Al}_{2} \mathrm{O}_{3}$ particles, CNTs do not find difference in associating itself with nano- $\mathrm{Al}_{2} \mathrm{O}_{3}$ [207]. Surface energy of CNTs range about $0.2 \mathrm{~J} / \mathrm{m}^{2}$, whereas $\mathrm{Al}_{2} \mathrm{O}_{3}$ particles have surface energy of $\sim 1.59$ $\mathrm{J} / \mathrm{m}^{2}$ [194]. It has been shown that Al-graphite interface can bring down the energy in the $0.02-0.4 \mathrm{~J} / \mathrm{m}^{2}$ range [194]. Nano- $\mathrm{Al}_{2} \mathrm{O}_{3}$ powder particles thereby aid dispersion of CNTs in the solid state mixing of nanocomposite powder [208]. Random surface availability therefore results in the uniform dispersion of CNTs. Distribution of CNTs in the A4C-SD and $\mathrm{A} 8 \mathrm{C}-\mathrm{SD}$ powder feedstock as a function of their aspect ratio is presented in Fig. 4.4 and Fig. 4.5 respectively. Figure 4.4 and Fig. 4.5 show non-preferential distribution of 
Table 4.1: Initial powder size, spray drying treatment and adopted nomenclature

\begin{tabular}{|c|c|c|c|c|c|}
\hline Initial Powders & $\begin{array}{c}\text { Powder Treatment } \\
\text {-Step } 1\end{array}$ & $\begin{array}{c}\text { Powder } \\
\text { Treatment } \\
\text {-Step 2 } \\
\end{array}$ & Resulting Powder & CNT Dispersion & $\begin{array}{l}\text { Plasma Sprayed } \\
\text { Coating } \\
\text { Nomenclature } \\
\end{array}$ \\
\hline \multirow{4}{*}{$\begin{array}{c}\mathrm{Al}_{2} \mathrm{O}_{3}(150 \mathrm{~nm} \\
\text { particle size) } \\
\\
\mathrm{CNTs} \\
\text { (Multi walled, OD } \\
40-70 \mathrm{~nm}, 0.5-2.0 \\
\quad \mu \mathrm{m} \text { long) }\end{array}$} & $\begin{array}{c}\text { Spray Drying of } \mathrm{Al}_{2} \mathrm{O}_{3} \\
\text { (agglomerate } \sim 15-60 \mu \mathrm{m})\end{array} \mid$ & --- & $\begin{array}{c}\boldsymbol{A - S D} \\
\left(\mathrm{Al}_{2} \mathrm{O}_{3} \text { agglomerate }\right. \\
15-60 \mu \mathrm{m})\end{array}$ & --- & $\begin{array}{c}\text { A-SD Coating } \\
\left(\mathrm{Al}_{2} \mathrm{O}_{3}\right)\end{array}$ \\
\hline & $\begin{array}{c}\text { Spray Drying of } \mathrm{Al}_{2} \mathrm{O}_{3} \\
(\text { agglomerate } \sim 15-60 \mu \mathrm{m})\end{array}$ & $\begin{array}{c}\text { Blending with } \\
4 \text { wt. } \% \text { CNTs } \\
\text { for } 24 \mathrm{~h}\end{array}$ & $\begin{array}{c}\boldsymbol{A} 4 \mathrm{C}-\boldsymbol{B} \\
\left(\mathrm{Al}_{2} \mathrm{O}_{3}-4 w t . \% \mathrm{CNT}\right. \\
\text { agglomerate } \\
\sim 15-60 \mu \mathrm{m})\end{array}$ & $\begin{array}{l}\text { Onto surface and } \\
\text { dome cavity }\end{array}$ & $\begin{array}{c}\text { A4C-B Coating } \\
\left(\mathrm{Al}_{2} \mathrm{O}_{3}-4 \text { wt.\% CNT }\right)\end{array}$ \\
\hline & $\begin{array}{c}\text { Spray Drying of } \mathrm{Al}_{2} \mathrm{O}_{3} \\
\text { and } \\
4 \text { wt.\% CNTs } \\
\text { (agglomerate } \sim 15-60 \mu \mathrm{m})\end{array}$ & --- & $\begin{array}{c}\mathrm{A} 4 \mathrm{C}-\mathrm{SD}\left(\mathrm{Al}_{2} \mathrm{O}_{3}-\right. \\
\quad 4 w t . \% \mathrm{CNT} \\
\text { agglomerate } \\
\sim 15-60 \mu \mathrm{m})\end{array}$ & $\begin{array}{l}\text { Throughout the } \\
\text { powder } \\
\text { agglomerate }\end{array}$ & $\begin{array}{c}\text { A4C-SD Coating } \\
\left(\mathrm{Al}_{2} \mathrm{O}_{3}-\right. \\
4 w t . \% \mathrm{CNT})\end{array}$ \\
\hline & $\begin{array}{c}\text { Spray Drying of } \mathrm{Al}_{2} \mathrm{O}_{3} \\
\text { and } \\
8 \text { wt.\% CNTs } \\
\text { (agglomerate } \sim 15-60 \mu \mathrm{m})\end{array}$ & --- & $\begin{array}{l}\text { A8C-SD }\left(\mathrm{Al}_{2} \mathrm{O}_{3}-\right. \\
\quad 8 w t . \% \mathrm{CNT} \\
\text { agglomerate } \\
\sim 15-60 \mu \mathrm{m})\end{array}$ & $\begin{array}{l}\text { Throughout the } \\
\text { powder } \\
\text { agglomerate }\end{array}$ & $\begin{array}{c}\text { A8C-SD Coating } \\
\left(\mathrm{Al}_{2} \mathrm{O}_{3}-\right. \\
8 w t . \% \mathrm{CNT})\end{array}$ \\
\hline
\end{tabular}


a)
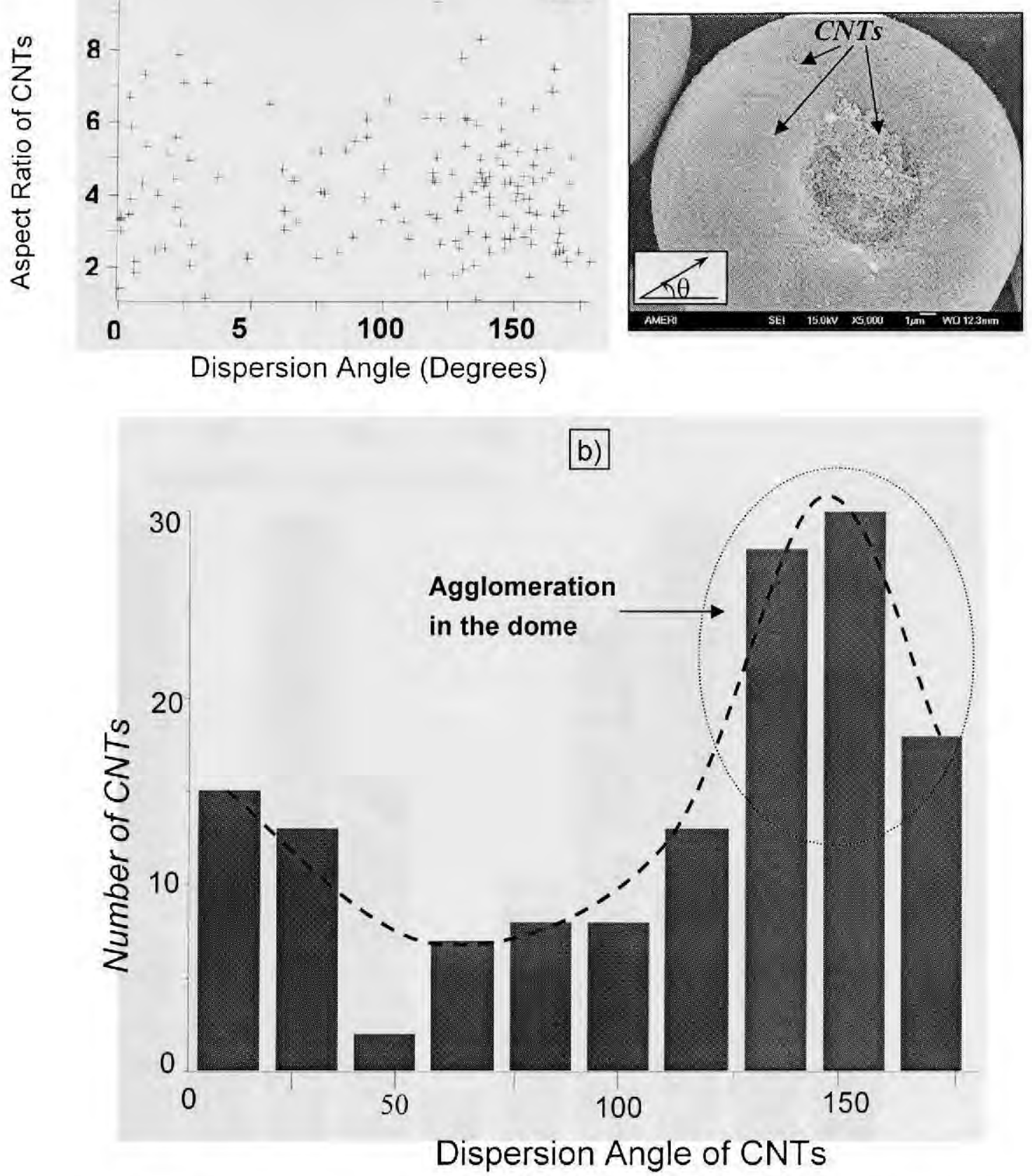

Fig. 4.3: a) Scattergram and representative micrograph describing CNT dispersion angle with respect to aspect ratio, and b) Histogram of $\mathrm{A} 4 \mathrm{C}-\mathrm{B}$ powder depicting agglomeration of CNTs in the dome cavity of the powder agglomerate. 

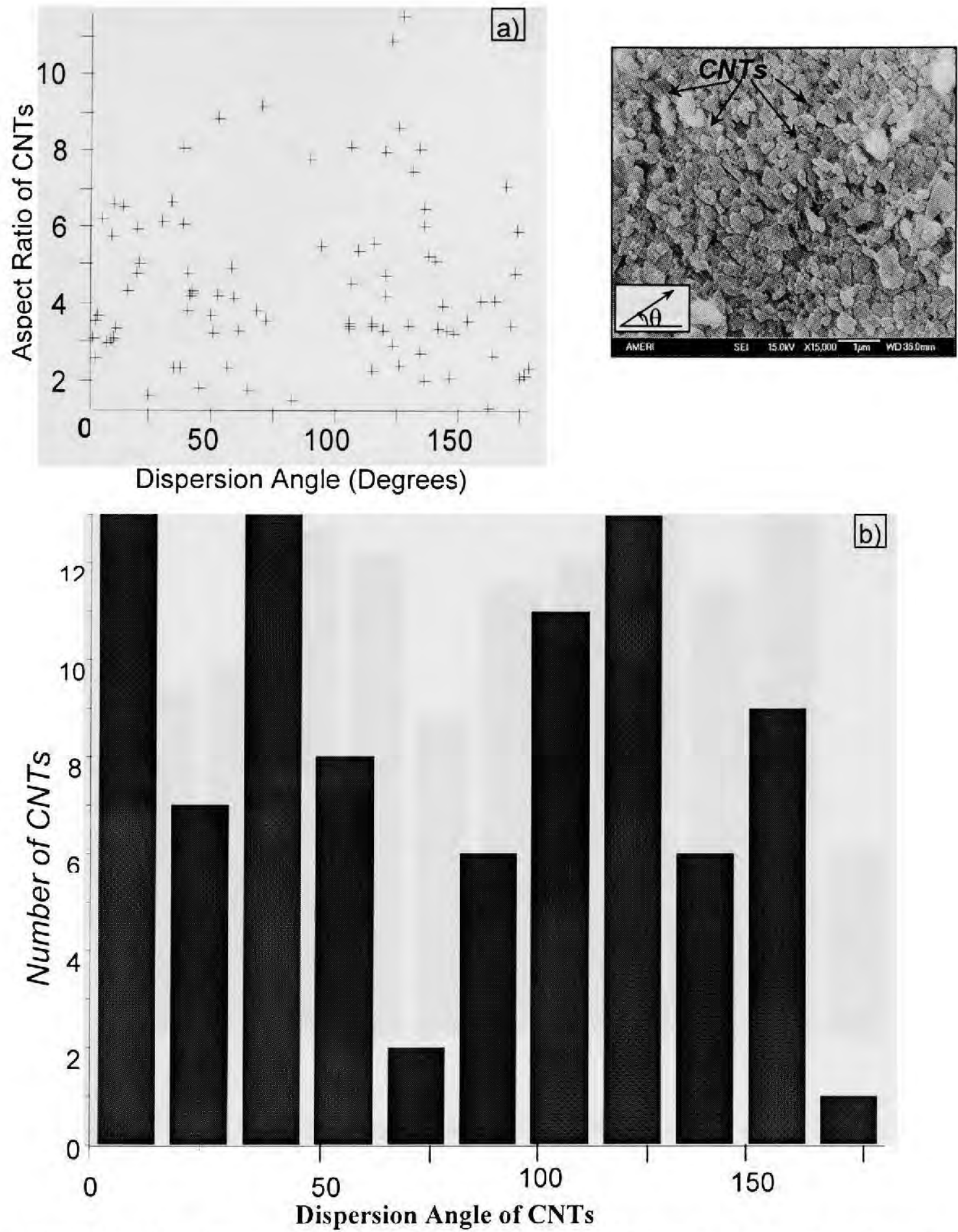

Fig. 4.4: a) Scattergram and representative micrograph describing CNT dispersion angle with respect to aspect ratio, and b) Histogram of A4C-SD powder depicting dispersion angle with respect to the number of CNTs in the powder agglomerate. 

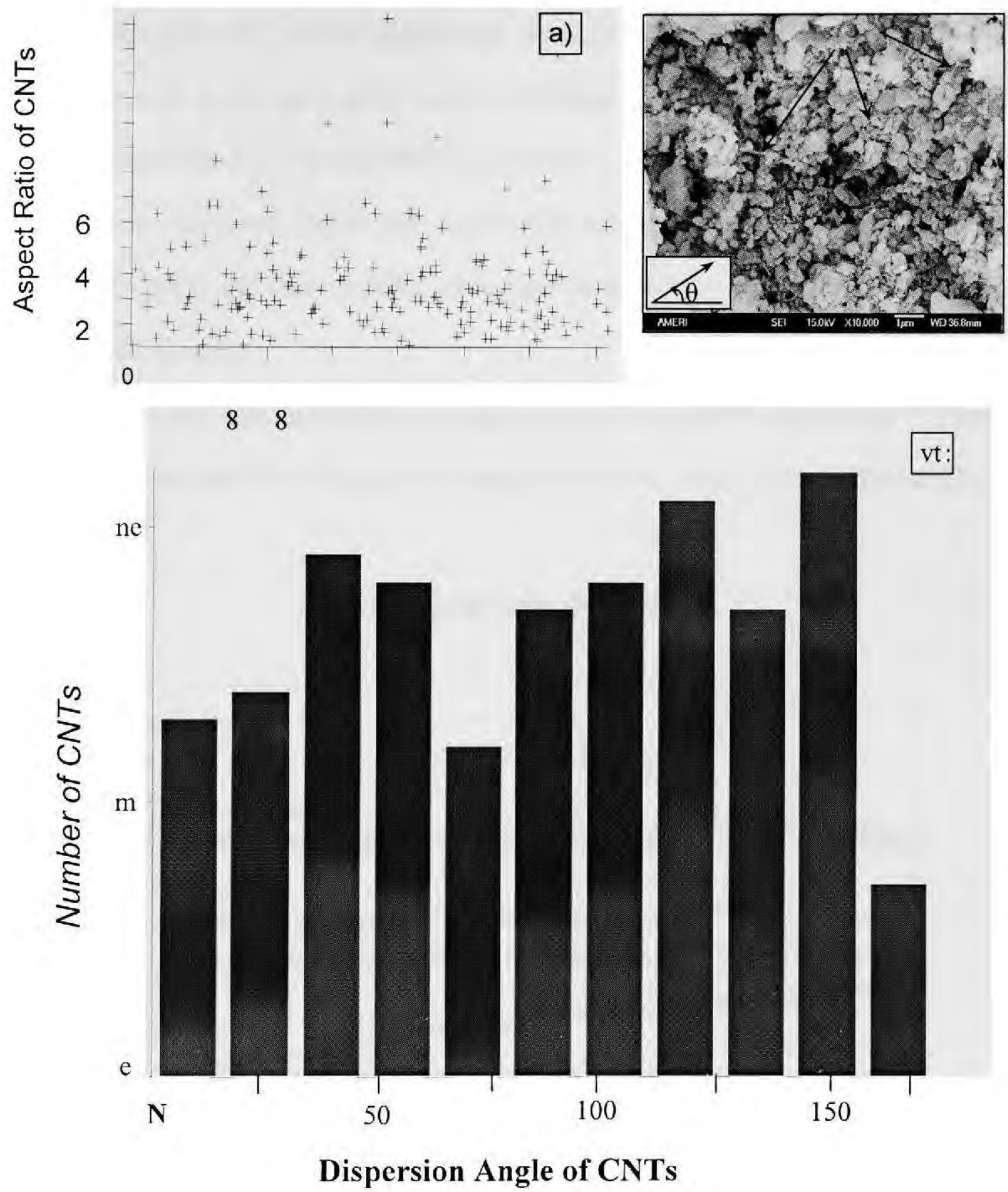

Fig. 4.5: a) Scattergram and representative micrograph describing CNT dispersion angle with respect to aspect ratio, and b) Histogram of A8C-SD powder depicting dispersion angle with respect to the number of CNTs in the powder agglomerate. 
CNTs throughout the powder agglomerate. Visually no bundles/agglomerates of CNTs were observed in the spray dried powder validating the non-preferred sticking sites for CNTs. Fractured A4C-SD and A8C-SD powders displayed excellent CNT dispersion in the core of spray dried agglomerate. Aspect ratio is helpful in understanding the pinning effect of CNTs, and corresponding dispersion angle suffices the reinforcement in the subsequent direction.

An initial signature of Raman spectrum was acquired for the powder feedstock, Fig. 4.6, to be able to compare the retention of CNT structure in the plasma sprayed

\section{Raman Spectrum of Powders}

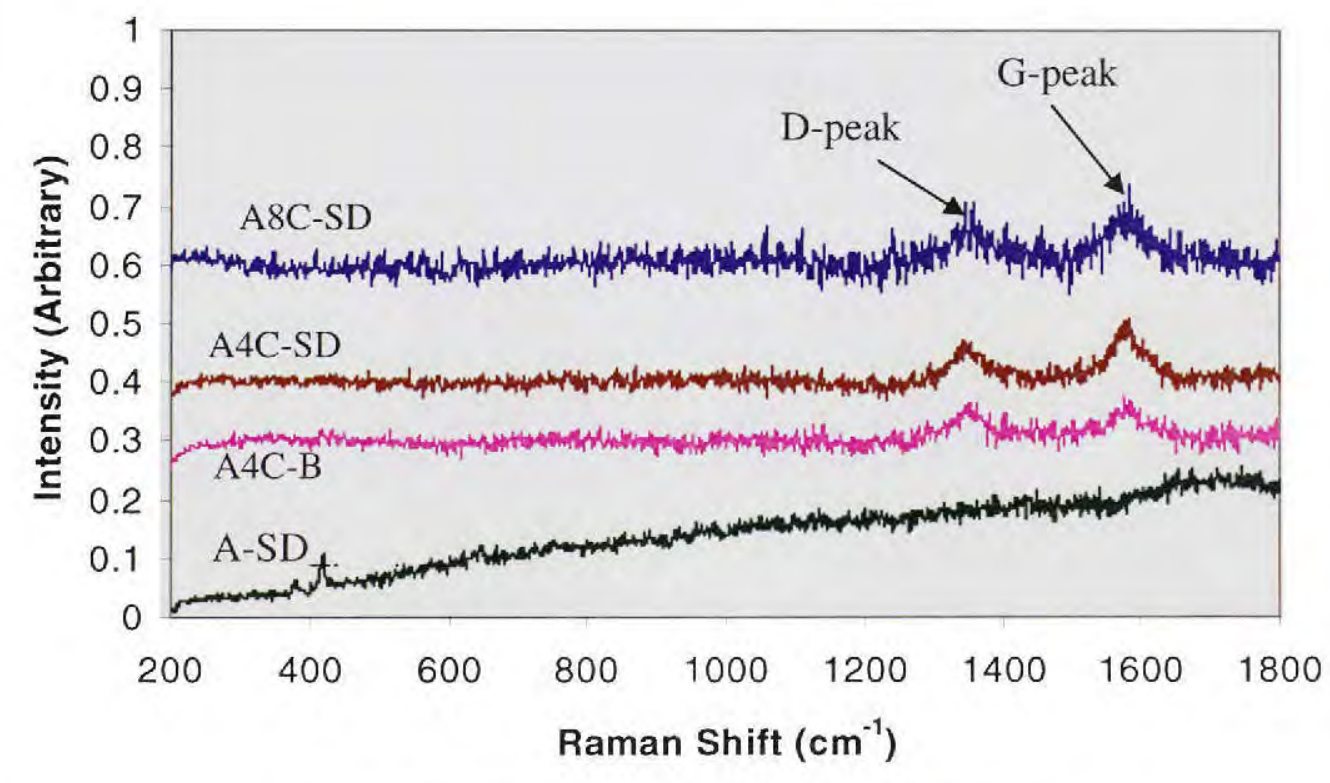

$A-S D-A 4 C-B-A 4 C-S D-A 8 C-S D$

Fig. 4.6: Raman spectrum of the initial powder feedstock (before plasma spraying). D and G peaks correspond to defect and graphitic structure of CNT. 
coatings. D and G peaks correspond to defect and graphitic peaks of CNT $[42,178]$. The presence of $\mathrm{G}$ peaks in the plasma sprayed coatings will confirm the presence of CNT structure. Since high temperature and impact associated with plasma spraying might damage the CNTs during the processing, an initial Raman spectrum assists in realizing importance of optimized plasma spray parameters in retaining CNTs in the plasma sprayed coatings.

\subsection{Optimization of Plasma Processing Parameters}

First step is to optimize the plasma spraying parameters in order to achieve uniform coating thickness and required microstructure for improved fracture toughness. Optimized processing of spray dried composite powder was required to result controlled melting of the surface and solid state sintering of core, Fig. 4.7.

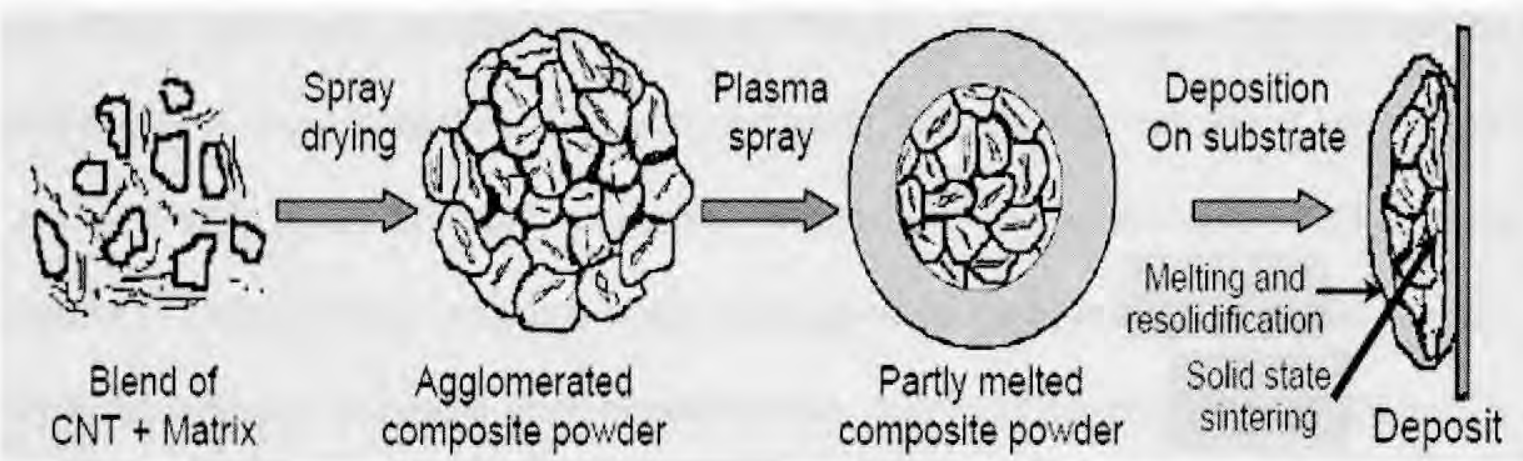

Fig. 4.7: Optimized plasma spraying to result surface melting and resolidification and core consolidation via solid state sintering. 
Surface melting and resolidification results metallurgical consolidation of splats, whereas solid state sintering of core retains the nanocrystalline nature of the powder feedstock. Increasing the power-input (from the optimized condition) will increase the degree of melting and the nanocrystalline nature of the powder may not be retained in the processed coating. On the other hand reducing the power input will not result proper consolidation between splats and the integrity of the coating may not be achieved. Thereby, plasma-processing parameters are optimized in terms of bimodal microstructure in the matrix and fracture toughness of resultant coatings. Bimodal microstructure is characterized through fully melted structure (surface melting and resolidification) and partially melted structure (solid state sintering of the core), Fig. 4.7. Partially melted/solid state sintered (PM) grains provide ductility by grain shearing, whereas fully melted (FM) regions provide strength owing to dense structure.

Spray-dried nano- $\mathrm{Al}_{2} \mathrm{O}_{3}\left(\mathrm{n}-\mathrm{Al}_{2} \mathrm{O}_{3}\right.$ : ASD powder) was used as the starting powder to obtain optimized processing conditions. Purpose of using nano $\mathrm{Al}_{2} \mathrm{O}_{3}$ is to take advantage of nanocrystalline grain with enhanced strength and ductility. Conventional micro ceramic grains are brittle in nature, whereas nanocrystalline ceramics show grain boundary shear sliding. Moreover crack propagation is more tortuous in nanocrystalline material owing to enhanced grain boundary area, which enhances the energy absorption during impact. Table 4.2 differentiates deformation mechanisms in micro and nanocrystalline ceramics. 
Table 4.2: Toughening in micro- and nano-crystalline grains

\begin{tabular}{|c|c|c|}
\hline Toughening of Ceramic & Conventional Micro Grain & Nanocrystalline Grain \\
\hline $\begin{array}{c}\text { Deformation } \\
\text { Mechanism }\end{array}$ & $\begin{array}{c}\text { Limited Plastic } \\
\text { Deformation: Brittle }\end{array}$ & $\begin{array}{c}\text { Deformation via Cracking } \\
\text { and Shear Sliding along } \\
\text { Grain Boundaries }\end{array}$ \\
\hline Crack Path & Less Tortuous & More Tortuous \\
\hline
\end{tabular}

\subsubsection{Optimization of Plasma Parameters on $\mathrm{n}_{-} \mathrm{Al}_{2} \underline{\mathrm{O}}_{3}$ Coating}

Overall plasma spraying parameters utilized in the optimization of depositing n$\mathrm{Al}_{2} \mathrm{O}_{3}$ coating are listed in Table 4.3. Processing parameters for plasma spraying of A-SD powder were selected based on earlier experience and exiting literature $[39,118,209$ 211]. Uniform coating thickness is observed in the plasma sprayed ASD coatings, as presented in Fig. 4.8. Gas flow rate is expressed in standard liters per minute (slm). Powder feed rate is expressed in rpm (rounds per minute) of the rotating wheel in the Praxair (model \#1264) powder feeder. The conversion of $\mathrm{rpm}$ to the feed rate in $\mathrm{g} / \mathrm{min}$ depends on the volume of powder being fed (in c.c.) per minute at specified speed of feeding wheel (in rpm).

It must be noted that slight tweaking of plasma parameters was required in order to obtain a uniform coating. Practical difficulties such as non-deposition of coating with lower plasma power required change in the carrier gas velocity and feed rate as observed in Table 4.3. Lower carrier gas flow rate increase the residence time of particles in plasma plume, so higher degree of melting occurs for powder particle. Similarly, lower feed rate converts to small amount of powder in same plasma plume volume. Thereby lower feed rate and lower carrier gas flow rate help coating deposition at low power. An 
overall picture of the plasma parameters and the resultant microstructure is presented in Fig. 4.9.

Coating thicknesses of $300,500,400$ and $120 \mu \mathrm{m}$ were obtained for the samples A, B, C and D respectively as shown in Fig. 4.10 a-d. Coatings are denser with increasing power, whereas lower power processed coatings depict increasing solid-state sintered region (Fig. 4.9). Image Pro imaging software dictated dense coatings (porosity between $13-18 \%)$ with varying degree of differential structure as explained in the consequent section.

Powder treatment (spray drying) and control of plasma parameters results in two distinct regions, viz. partially melted /solid-state sintered (PM), and fully melted and resolidified $(\boldsymbol{F M})$ structure in the coating with varying plasma power. This bimodal structure is attributed to $30-45$ percent porosity of spray dried powder feedstock [94] coupled with optimized plasma parameters. Velocity $(v)$ and temperature $(T)$ are the controlling parameters deciding the microstructure of the sprayed coatings. Velocity corresponds to the kinetic energy attained by inflight particle. This in turn decides the degree of flattening and subsequent densification of the powder particle upon impact. The degree of melting is decided by the temperature attained by inflight particles. Thus the interplay of kinetic- (through velocity) and thermal- energy (via temperature) is critical in the generation of the microstructure. Thereby, inflight sensor monitoring of velocity and temperature (of the inflight powders) serve as controlling factors in optimizing the plasma processing parameters. 


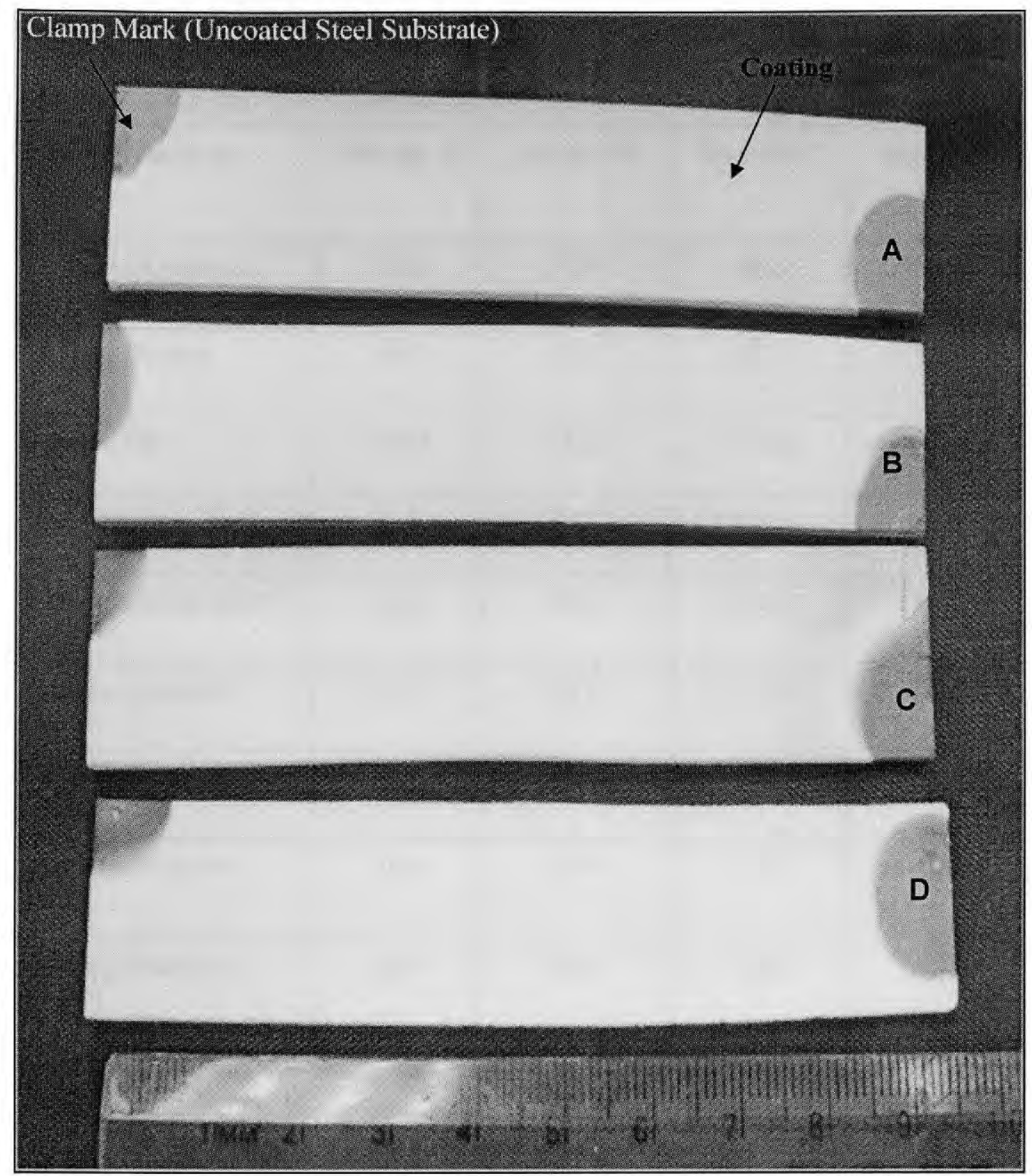

Fig. 4.8: Plasma sprayed coatings (from top: Sample A, B, C, and D respectively) on an AISI 1020 steel coupon of size $100 \times 19 \times 3.1 \mathrm{~mm}^{3}$. Coating thickness varies between 120- $500 \mu \mathrm{m}$. 
Table 4.3: Plasma spraying parameters used for spraying $\mathrm{Al}_{2} \mathrm{O}_{3}$

\begin{tabular}{|l|c|c|c|c|}
\hline \multicolumn{1}{|c|}{ Parameters } & Sample A & Sample B & Sample C & Sample D \\
\hline Current (Amperes) & 780 & 727 & 642 & 512 \\
\hline Voltage (Volts) & 39.5 & 38.3 & 37.3 & 36.2 \\
\hline Power (kW) & 30.81 & 27.84 & 23.95 & 18.53 \\
\hline Primary, Ar (slm*) & 32.1 & 32.1 & 32.1 & 32.1 \\
\hline Secondary, He (slm*) & 59.5 & 59.5 & 59.5 & 59.5 \\
\hline $\begin{array}{l}\text { Carrier, Ar (slm*) } \\
\text { Feed Rate (rpm) }\end{array}$ & 41.7 & 19.8 & 19.8 & 19.8 \\
\hline $\begin{array}{l}\text { Standoff Distance } \\
(\mathrm{mm})\end{array}$ & 100 & 100 & 100 & 100 \\
\hline $\begin{array}{l}\text { Coating Thickness } \\
(\mu \mathrm{m})\end{array}$ & 300 & 500 & 400 & 120 \\
\hline
\end{tabular}

*slm: standard liters per minute

Various plasma parameters required different powder feed and gas flow rates in order to deposit a coating under different plasma power conditions. Subsequent development of the bimodal phases is optimized by plasma spraying of spray dried nano $\mathrm{Al}_{2} \mathrm{O}_{3}$ powder feedstock. Consequently, the generated microstructure is characterized depending upon the bimodal structure and the mechanical properties (hardness and 
fracture toughness) attained by the plasma sprayed coatings. The role of initial powder treatment and the subsequent role of plasma parameters are discussed in the current section.

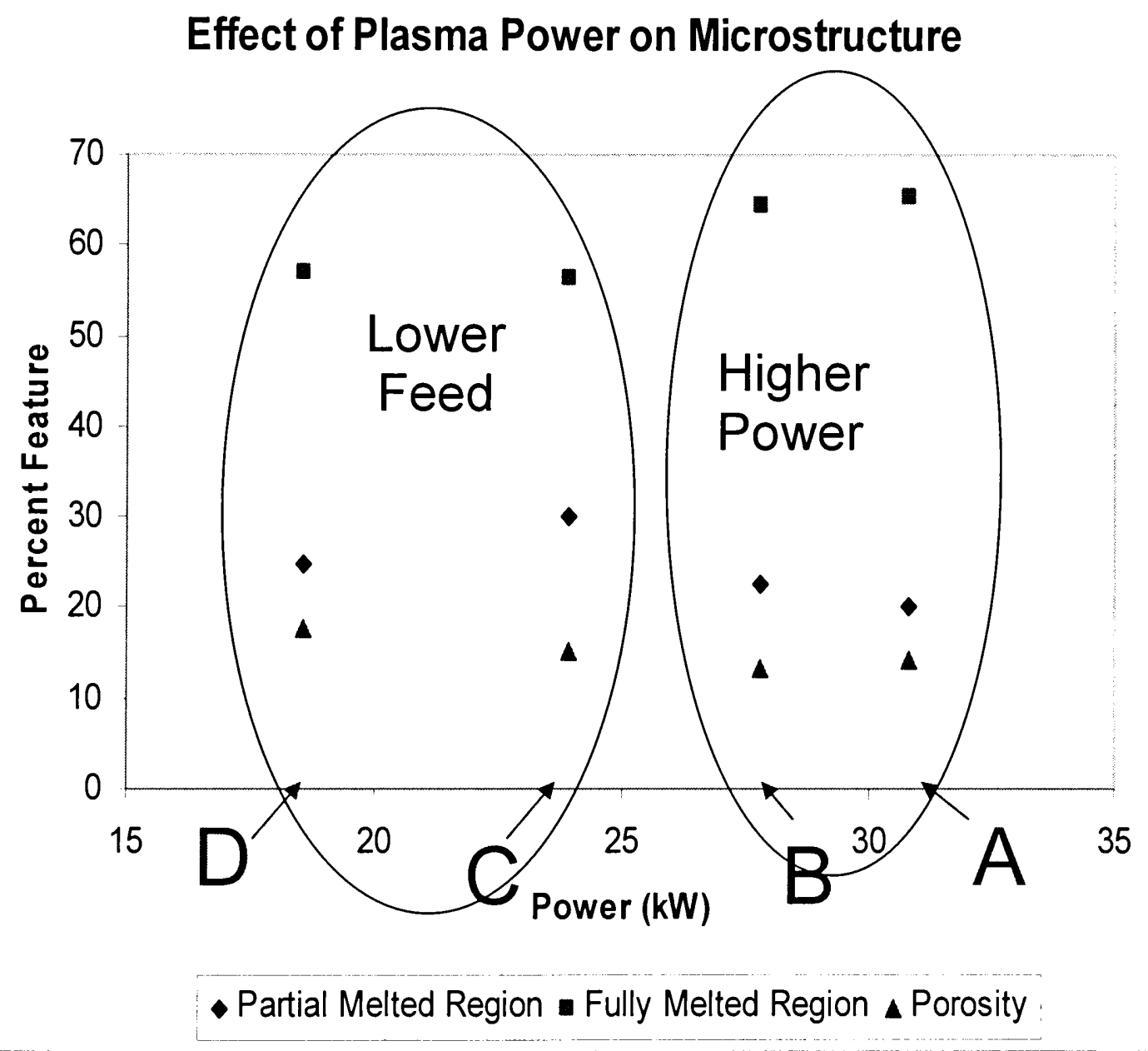

Fig. 4.9: Plasma spray parameter optimization showing relation of microstructural features of sprayed coatings with differential plasma parameter settings. 

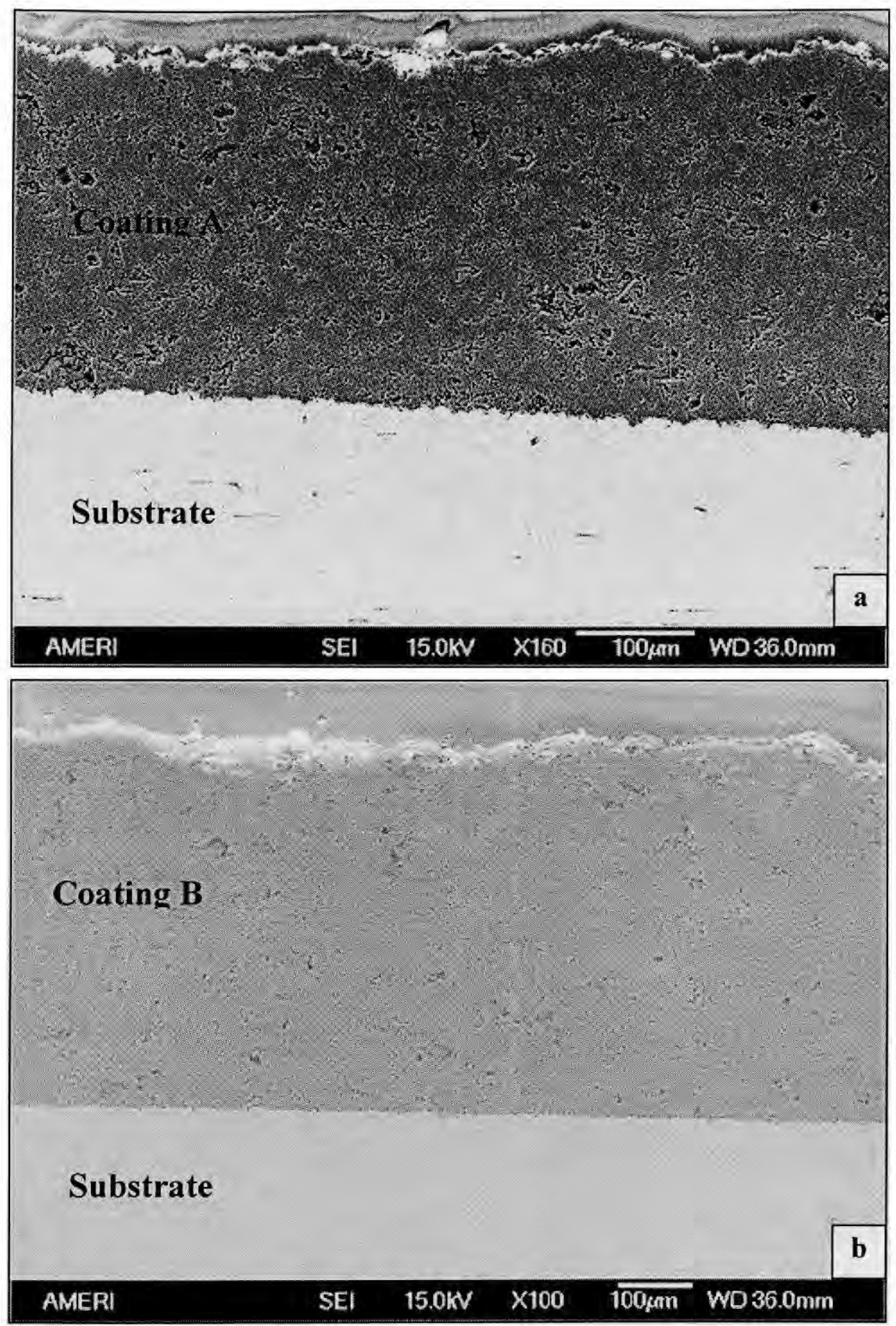

Fig. 4.10: Plasma sprayed $\mathrm{nAl}_{2} \mathrm{O}_{3}$ coating: a) Sample A, and b) Sample B from A-SD powder. 

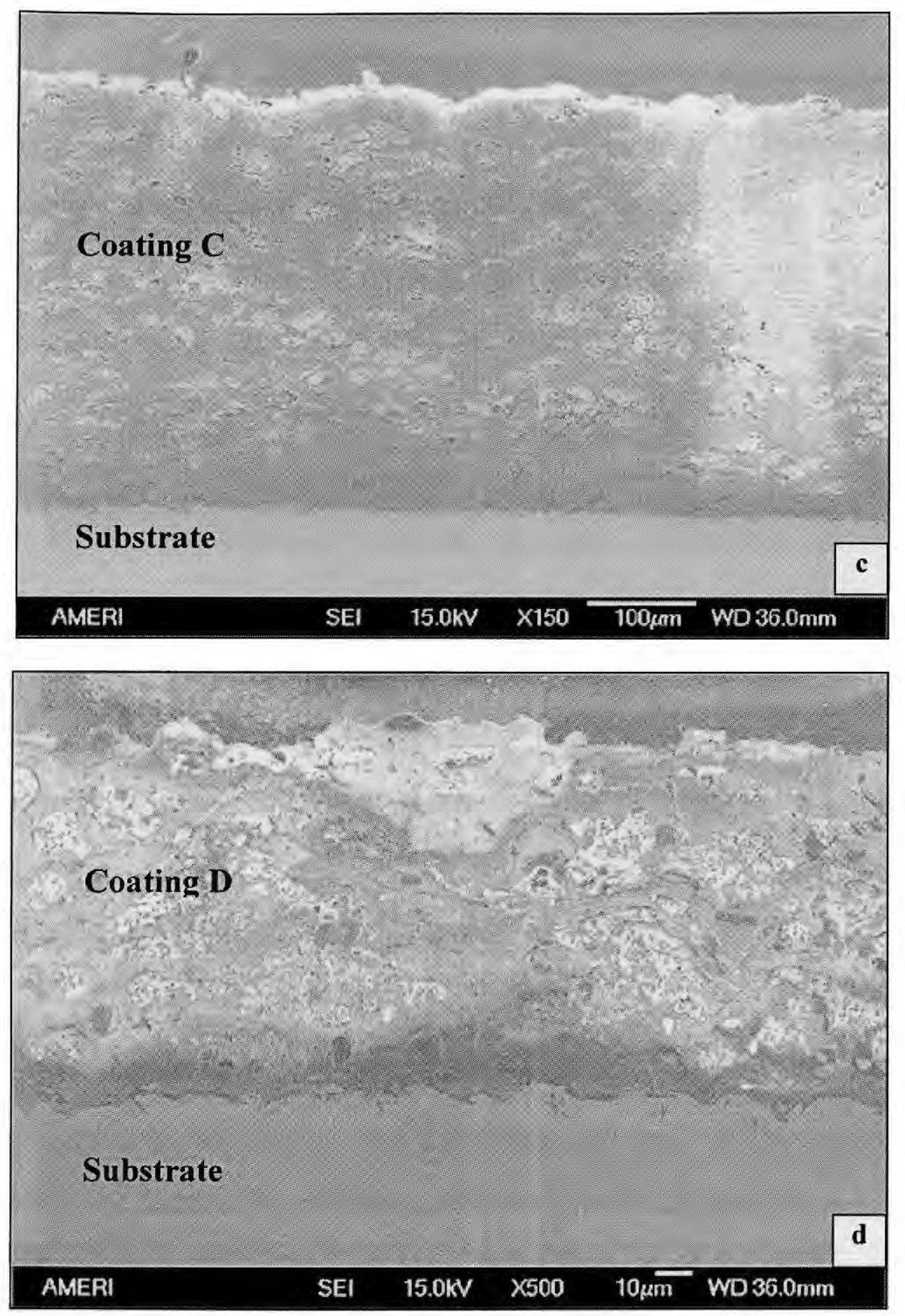

Fig. 4.10: Plasma sprayed $\mathrm{nAl}_{2} \mathrm{O}_{3}$ coating: c) Sample C, and d) Ssample D from A-SD powder. 
Owing to poor thermal conductivity of porous spray dried agglomerate, partial melting is sustained in the core whereas fully melted region is obtained on the surface. The surface-melting of powder particles results fully melted and re-solidified regions whereas the core of the powder agglomerate gets sintered in solid state. Hence, solid state sintering of the $\mathrm{Al}_{2} \mathrm{O}_{3}$ nano-particles occurs in the core region without destroying the nano nature of the starting powders. Thus, the matrix results in bimodal grain structure, i.e. fully melted and resolidified outer region and partially melted/solid-state sintered core region. Partially melted/sintered region helps in distributing the shock energy experienced during an impact, and helps deflecting crack resisting its propagation. Fully melted regions imparts strength and binding integrity to the composite coating $[94,114]$.

Increasing power shows increased FM zone, and increased grain size in the PM region, Fig. 4.11 through Fig. 4.14. Grain size is highest $(\sim 1 \mu \mathrm{m})$ in the highest plasma power setting, Fig. 4.11, and decreases to $0.8 \mu \mathrm{m}$ (Fig. 4.12), to $0.5 \mu \mathrm{m}$, (Fig. 4.13) and to $0.35 \mu \mathrm{m}$ (Fig. 4.14) in Sample A, B, C and D respectively. Influence of plasma parameters on microstructural features is quantified in Table 4.4 and Fig. 4.9.

Fracture toughness of the coatings is measured using Anti's equation (eq. 2.1) from Vicker indentation at $200 \mathrm{~g}$ with a dwell time of $15 \mathrm{~s}$, Table 4.4 [59]. Radial crack generate upon unloading, and the crack is restrained when the crack tip stress intensity is balanced by the fracture toughness of the material to restrict its propagation as shown in Fig 4.15. Uniform bimodal microstructure distribution and highest fracture toughness $\left(\sim 3.29 \mathrm{MPa} \mathrm{m}^{1 / 2}\right)$ were obtained for sample $\mathrm{C}$ (Voltage: $37.3 \mathrm{~V}$, and Current: $\left.642 \mathrm{~A}\right)$. Hence, processing parameters from sample $\mathrm{C}$ were selected as optimized condition to spray $\mathrm{Al}_{2} \mathrm{O}_{3}-\mathrm{CNT}$ coatings for improved fracture toughness. 


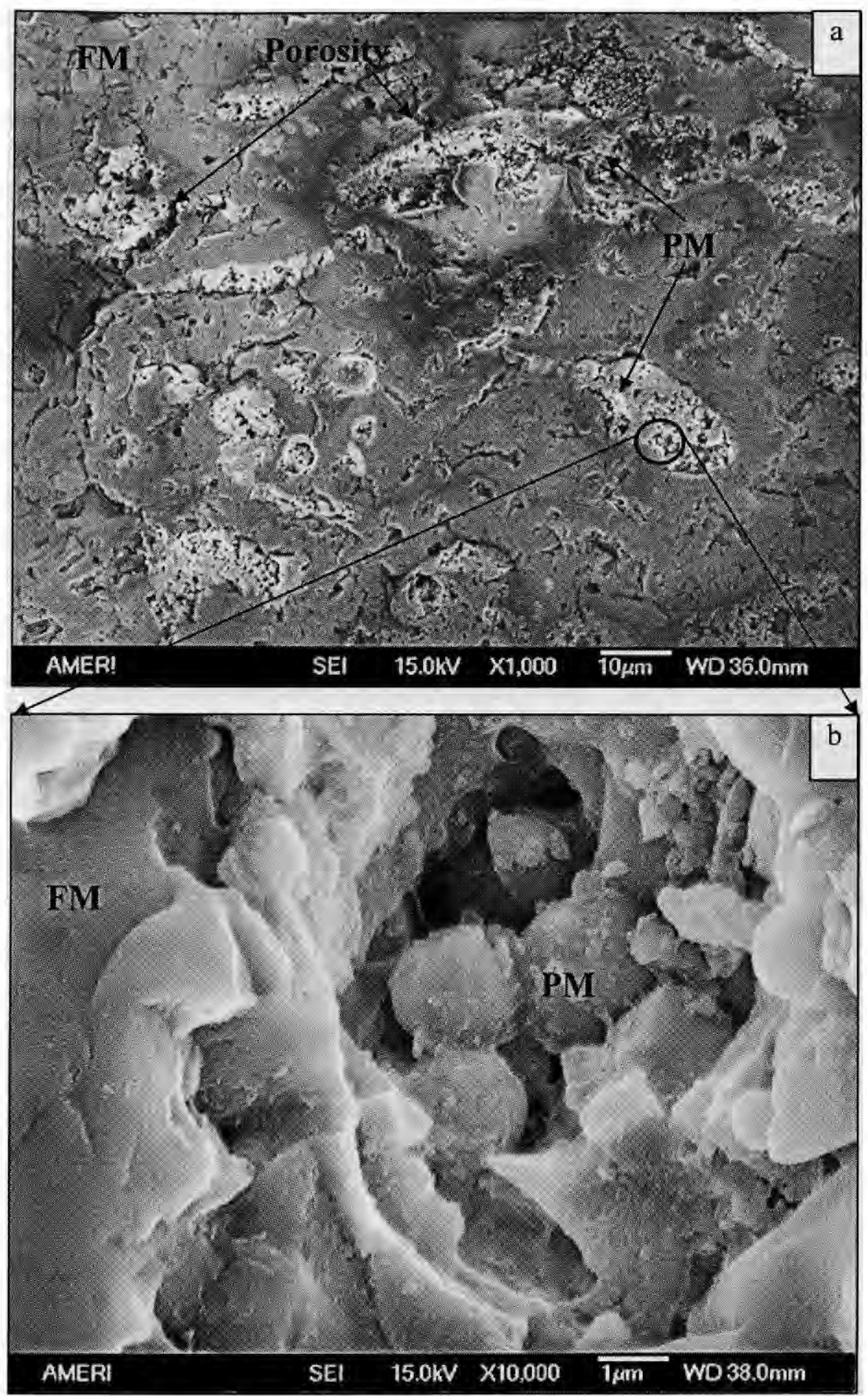

Fig. 4.11: Plasma sprayed $\mathrm{nAl}_{2} \mathrm{O}_{3}$ coating (Sample A) showing a) PM, FM and porosity distribution, and b) solid-state sintering of powders. Note that grain size is around $1 \mu \mathrm{m}$. 


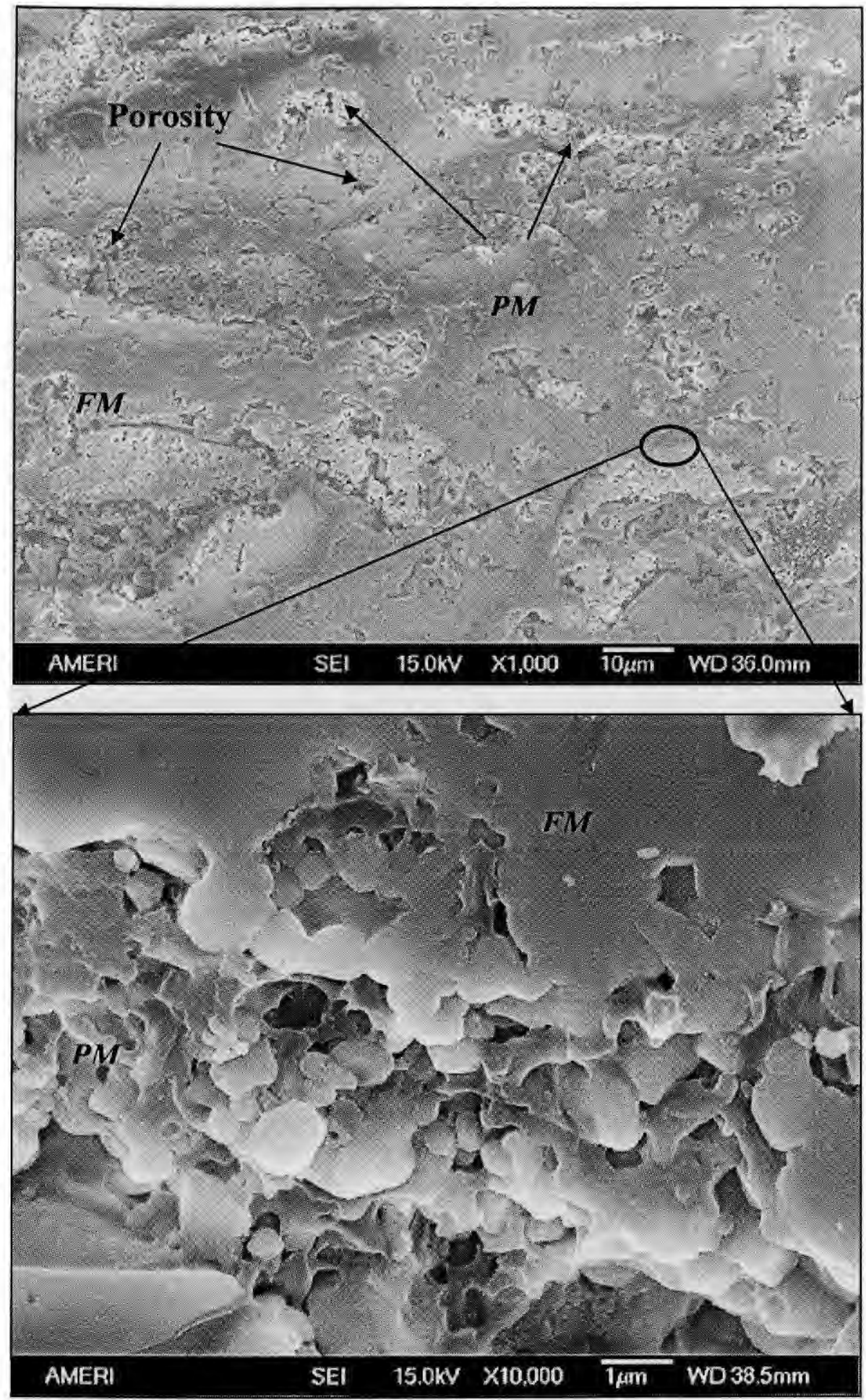

Fig. 4.12: Plasma sprayed $\mathrm{nAl}_{2} \mathrm{O}_{3}$ coating (Sample B) showing a) PM, FM and porosity distribution, and b) solid-state sintering of powders. Note that grain size is decreased to around $0.8 \mu \mathrm{m}$. 

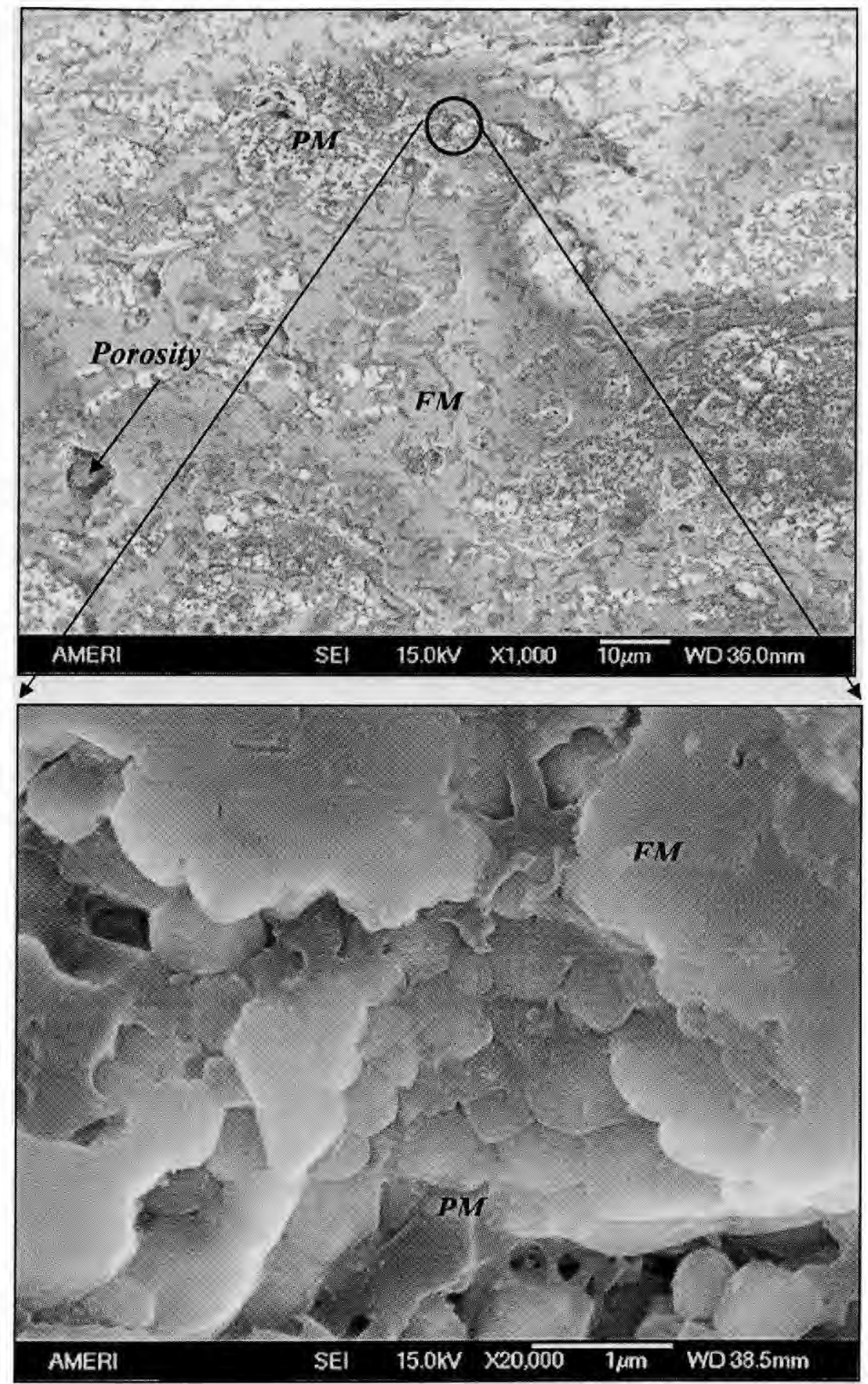

Fig. 4.13: Plasma sprayed $\mathrm{nAl}_{2} \mathrm{O}_{3}$ coating (Sample C) showing a) PM, FM and porosity distribution, and b) solid-state sintering of powders. Note that grain size is further decreased to around $0.5 \mu \mathrm{m}$. 


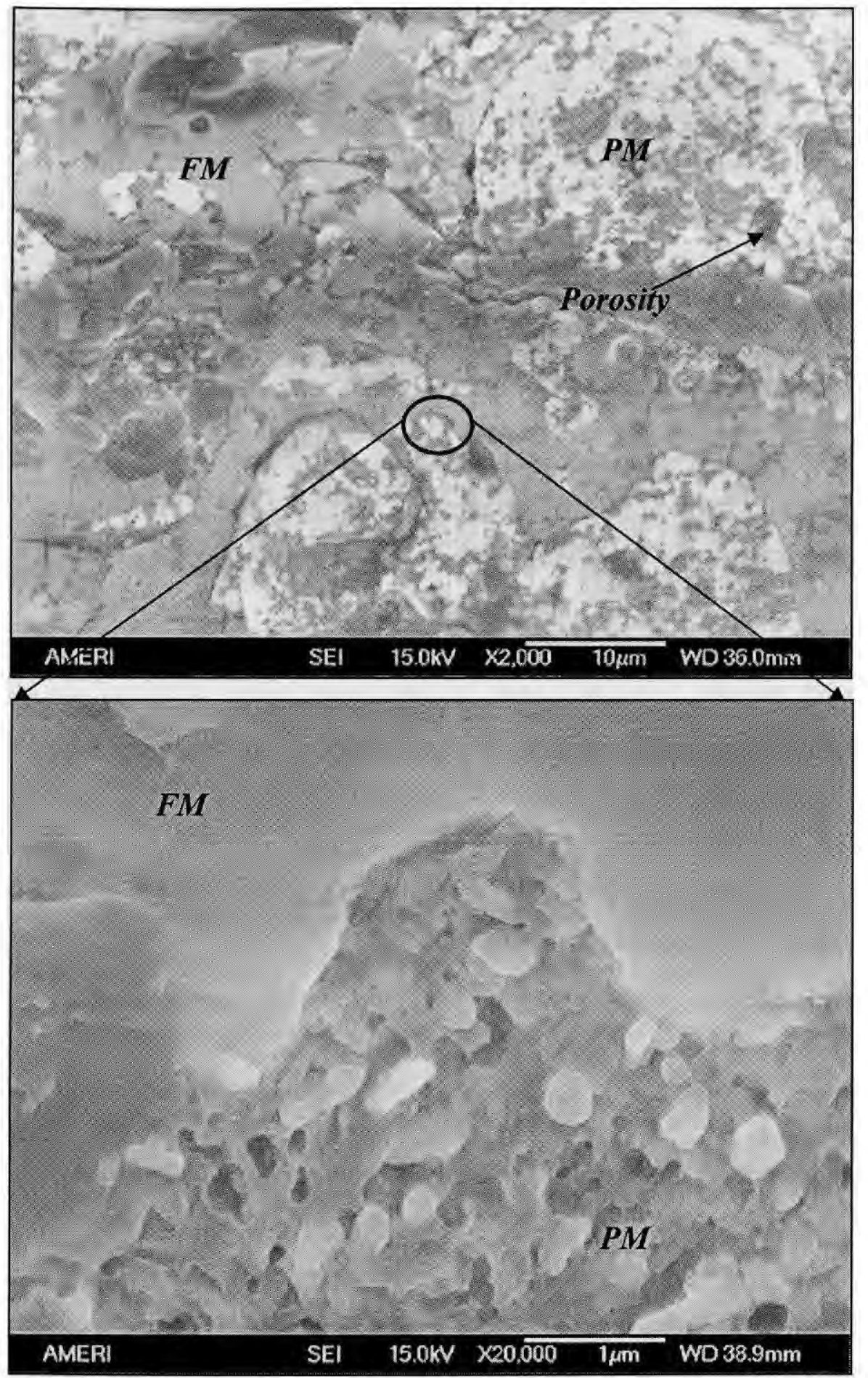

Fig. 4.14: Plasma sprayed $\mathrm{nAl}_{2} \mathrm{O}_{3}$ coating (Sample D) showing a) PM, FM and porosity distribution, and b) solid-state sintering of powders. Note that grain size is $\sim 0.35 \mu \mathrm{m}$. 


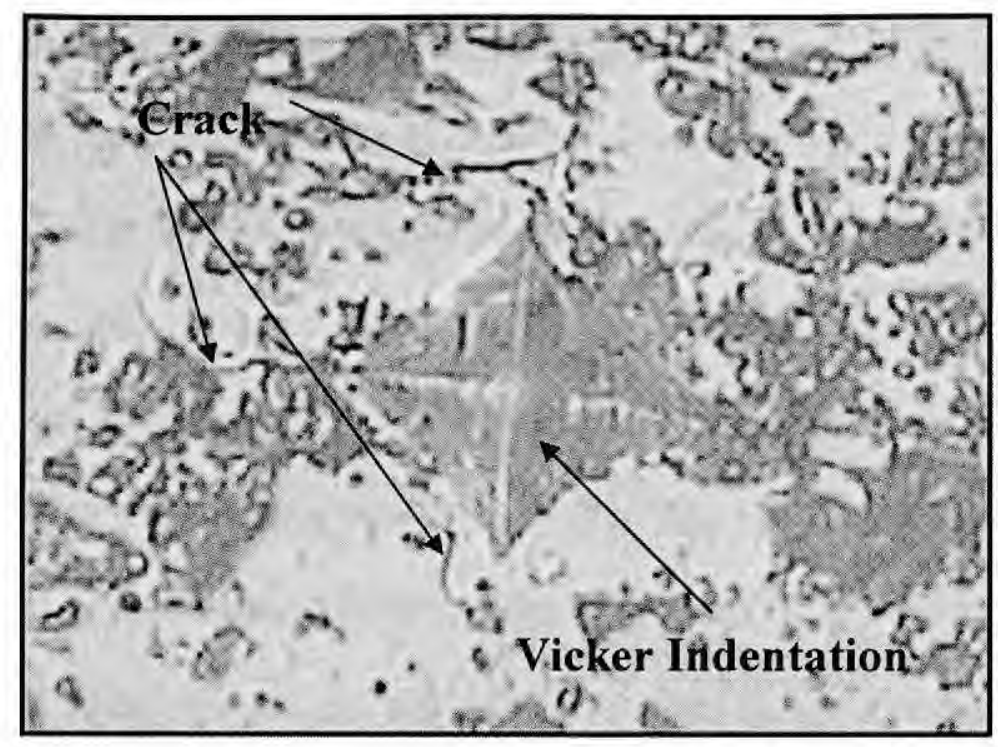

Fig. 4.15: Radial crack generation in plasma sprayed $n-\mathrm{Al}_{2} \mathrm{O}_{3}$ coating via Vicker indentation.

Table 4.4: Microstructural features and fracture toughness of plasma sprayed $\mathrm{nAl}_{2} \mathrm{O}_{3}$ coatings

\begin{tabular}{|l|c|c|c|c|c|}
\hline $\begin{array}{c}\text { Features } \rightarrow \\
\text { Coatings } \downarrow\end{array}$ & $\begin{array}{c}\text { FM Content } \\
\mathbf{( \% )}\end{array}$ & $\begin{array}{c}\text { PM Content } \\
\mathbf{( \% )}\end{array}$ & $\begin{array}{c}\text { Porosity } \\
\mathbf{( \% )}\end{array}$ & $\begin{array}{c}\text { Coating } \\
\text { Thickness } \\
\boldsymbol{\mu m}\end{array}$ & $\begin{array}{c}\text { Fracture } \\
\text { Toughness } \\
\mathbf{M P a ~ m}^{\mathbf{1} / \mathbf{2}}\end{array}$ \\
\hline Sample A & $65.3 \pm 2.9$ & $20.2 \pm 2.4$ & $14.3 \pm 1.2$ & 300 & $1.51-3.55$ \\
\hline Sample B & $64.5 \pm 2.3$ & $22.7 \pm 1.9$ & $13.3 \pm 0.0$ & 500 & $1.28-1.36$ \\
\hline Sample C & $56.5 \pm 1.8$ & $30.1 \pm 3.8$ & $15.0 \pm 2.5$ & 400 & $2.53-3.29$ \\
\hline Sample D & $56.9 \pm 1.6$ & $24.6 \pm 1.7$ & $17.7 \pm 1.0$ & 120 & $1.07-1.66$ \\
\hline
\end{tabular}




\subsection{Plasma Spraying of $n-\mathrm{Al}_{2} \mathrm{O}_{3}$ with Addition of CNTs}

A4C-B, A4C-SD, and A8C-SD powders were plasma sprayed with the plasma parameters listed in Table 4.5. It was observed that plasma parameters optimized for ASD coating (Sample C) were not sufficient to deposit a uniform CNT reinforced coating. Since CNTs are uniformly distributed in spray dried powders, they extract heat from $\mathrm{Al}_{2} \mathrm{O}_{3}$ particles due to their high thermal conductivity. Thereby higher power is required to melt $\mathrm{Al}_{2} \mathrm{O}_{3}$ particles and get uniformly thick coatings. Accuraspray inflight sensor data clearly elicits such observation and thereby higher power plasma parameters were utilized in order to accommodate deposition of a uniform coating (Table 4.5). Comparative inflight particle diagnostic data (temperature and velocity of inflight powder particles) for all samples is discussed in later section.

Table 4.5: Plasma parameters for spraying A4C-B, A4C-SD and A8C-SD coating

\begin{tabular}{|c|c|c|c|}
\hline $\begin{array}{c}\text { Coatings } \rightarrow \\
\text { Parameters } \downarrow\end{array}$ & A4C-B Coating & A4C-SD Coating & A8C-SD Coating \\
\hline Current (Amperes) & 630 & 778 & 761 \\
\hline Voltage (Volts) & 40.8 & 39.6 & 42.5 \\
\hline Power (kW) & 25.7 & 30.8 & 32.3 \\
\hline Primary, Ar (slm) & 32.1 & 32.1 & 32.1 \\
\hline Secondary, He (slm) & 59.5 & 59.5 & 59.5 \\
\hline Carrier, Ar (slm) & 19.8 & 19.8 & 19.8 \\
\hline Feed Rate (rpm) & 3.0 & 3.0 & 3.0 \\
\hline Standoff Distance $(\mathrm{mm})$ & 100 & 100 & 100 \\
\hline Coating Thickness $(\mu \mathrm{m})$ & 350 & 450 & 500 \\
\hline
\end{tabular}

* slm: standard liters per minute 
In this section, comparable plasma parameters, Table 4.5, are used for studying the role of CNT dispersion in generating a contrasting microstructure and mechanical properties. Plasma sprayed A-SD coating under optimized conditions (Sample C with highest fracture toughness) is taken as a reference for comparing the CNT reinforced $\mathrm{Al}_{2} \mathrm{O}_{3}$ coatings. Steel samples coated with A-SD, A4C-B, A4C-SD and A8C-SD are presented in Fig 4.16 a-d. A-SD coating is whitish (Fig. 4.16 a), A4C-B coating is grayish (Fig. 4.16 b), A4C-SD coating is dark-grayish (Fig. $4.16 \mathrm{c}$ ), whereas A8C-SD coating is blackish (Fig. $4.16 \mathrm{~d}$ ) in color. A-SD coating is white owing to the pure $\mathrm{Al}_{2} \mathrm{O}_{3}$ coating (Fig. 4.16a). Black specs and grayish color is observed in A4C-B coating (Fig. 4.16b) owing to agglomeration and dispersion of CNTs respectively. Uniform dark grayish nature of A4C-SD (Fig. 4.16c) is attributed to the uniform CNT dispersion in the coating. Uniformly black color of A8C-SD (Fig. 4.16d) is attributed to higher CNT content and their uniform dispersion. Overall microstructure generated in each CNT reinforced coating is discussed in following sections.

Table 4.6 shows the inflight particle diagnostic data for various plasma sprayed coatings. Temperature variation of different powders owing to CNT dispersion is clearly evinced in Table 4.6. Contrasting difference is attributed to role of high thermal conductivity of CNTs in the A4C-B powder (CNT agglomeration) and A4C-SD powder (CNT dispersion).

\subsubsection{Plasma Sprayed A4C-B Coating}

Fig. 4.17 shows plasma sprayed A4C-B nanocomposite coating on the AISI 1020 steel substrate. Uniform and homogeneous A4C-B nanocomposite coating of $\sim 350 \mu \mathrm{m}$ is 


\section{A-SD Coating}
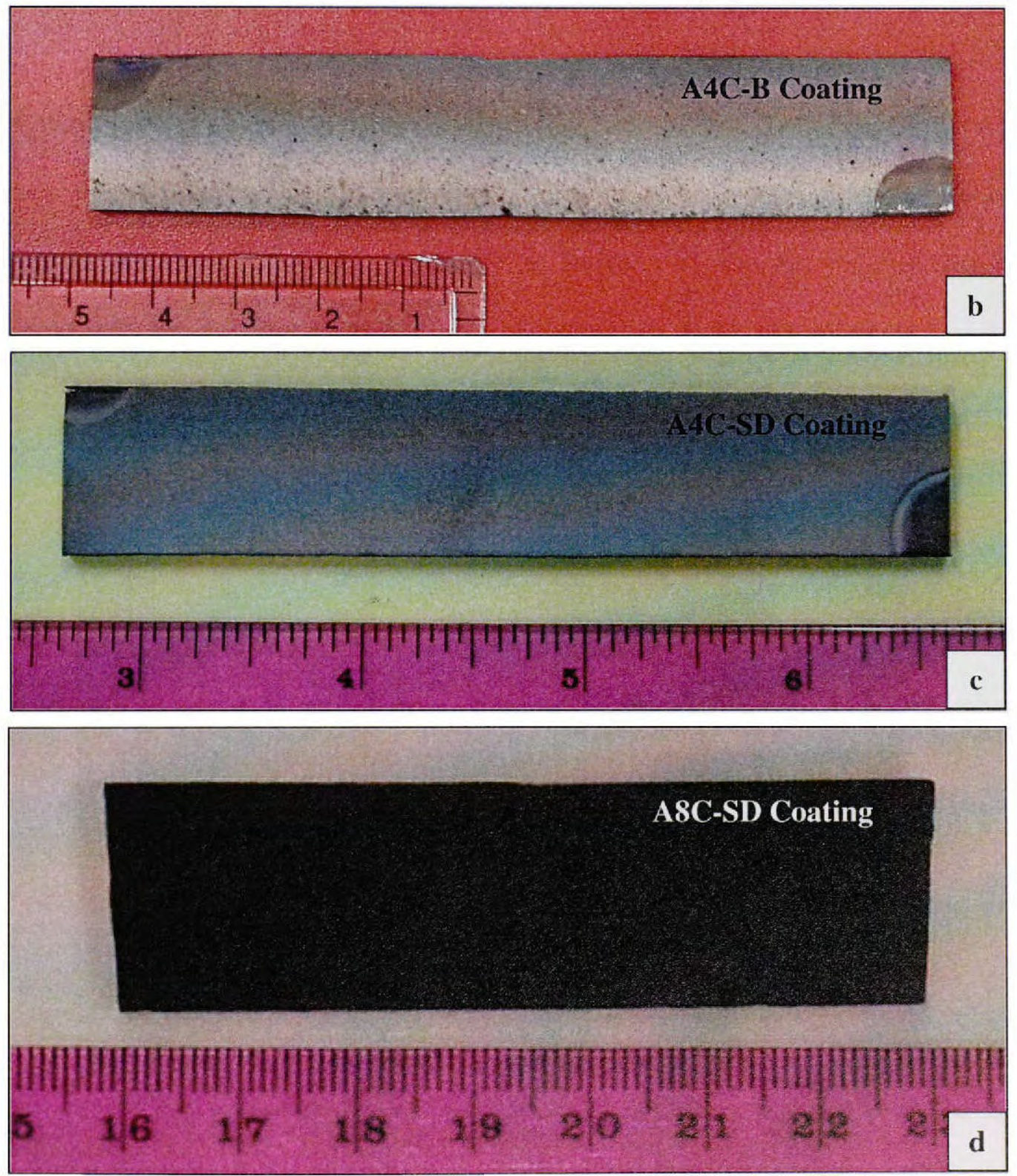

Fig. 4.16: Plasma sprayed a) A-SD, b) A4C-B, c) A4C-SD, and d) A8C-SD coating. 
Table 4.6: Inflight particle diagnostic data for various plasma sprayed coatings

\begin{tabular}{|c|c|c|c|c|}
\hline Coating & Temperature (K) & Velocity (m/s) & Dwell Time (s) & Cooling Rate (K/s) \\
\hline A-SD Coating & 2512 & 289 & $3.46 \times 10^{-4}$ & $7.26 \times 10^{6}$ \\
\hline A4C-B Coating & 2898 & 271 & $3.69 \times 10^{-4}$ & $7.85 \times 10^{6}$ \\
\hline A4C-SD Coating & 2332 & 244 & $4.10 \times 10^{-4}$ & $5.69 \times 10^{6}$ \\
\hline A C C-SD Coating & 2241 & 232 & $4.31 \times 10^{-4}$ & $5.20 \times 10^{6}$ \\
\hline
\end{tabular}

obtained. The coating is free from cracks and adherent to the substrate. Coating was removed from the substrate to obtain as a freestanding structure. Density of $3.40 \mathrm{~g} / \mathrm{cc}$ (87.2\% dense as compared to theoretical density of 3.90 for $\mathrm{Al}_{2} \mathrm{O}_{3}-4$ wt. \% CNT composite) was obtained through Archimedes water immersion technique.

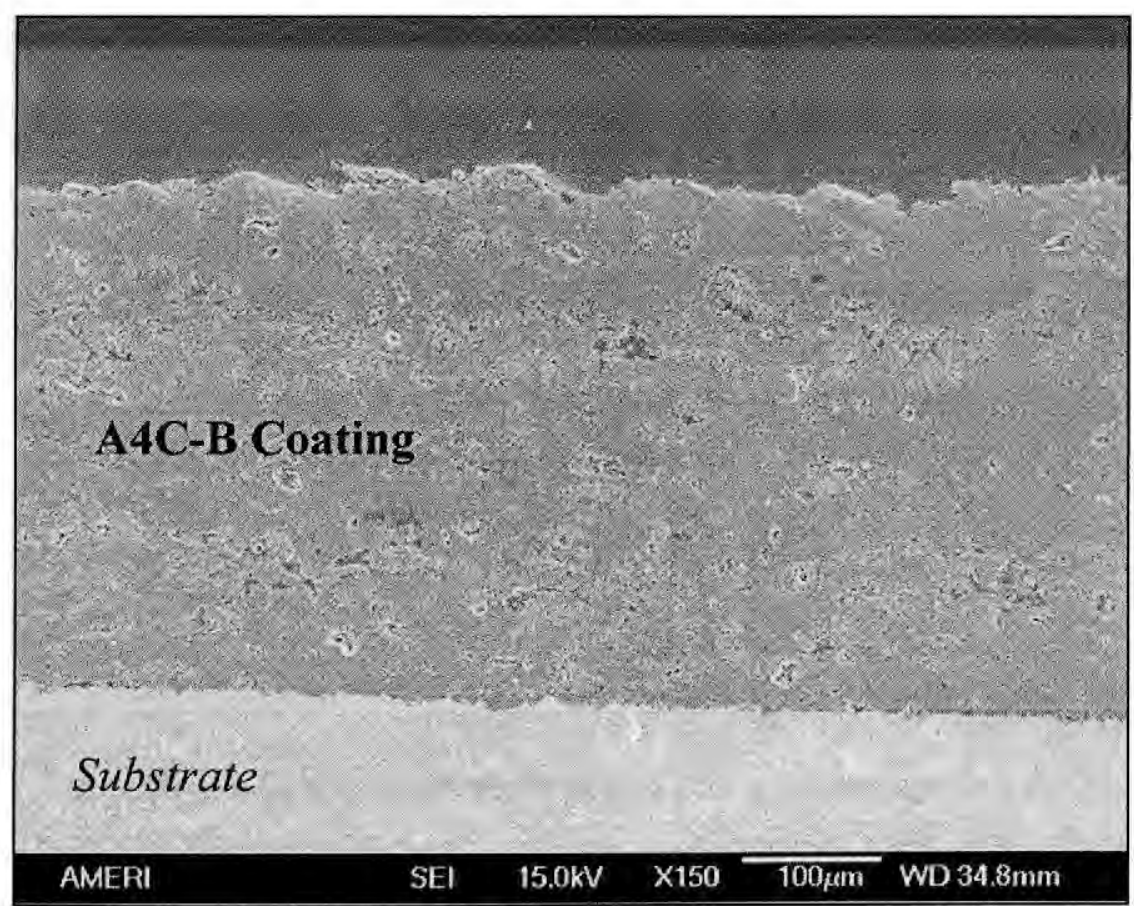

Fig. 4.17: Cross-sectional image of A4C-B coating showing uniform and adherent coating. 
At higher magnification, differential microstructure with fully melted and resolidified (FM) and partially-melted and solid state sintered (PM) regions is observed in Fig. 4.18. This microstructure is similar to that of optimized plasma sprayed A-SD coating (Fig. 4.13).

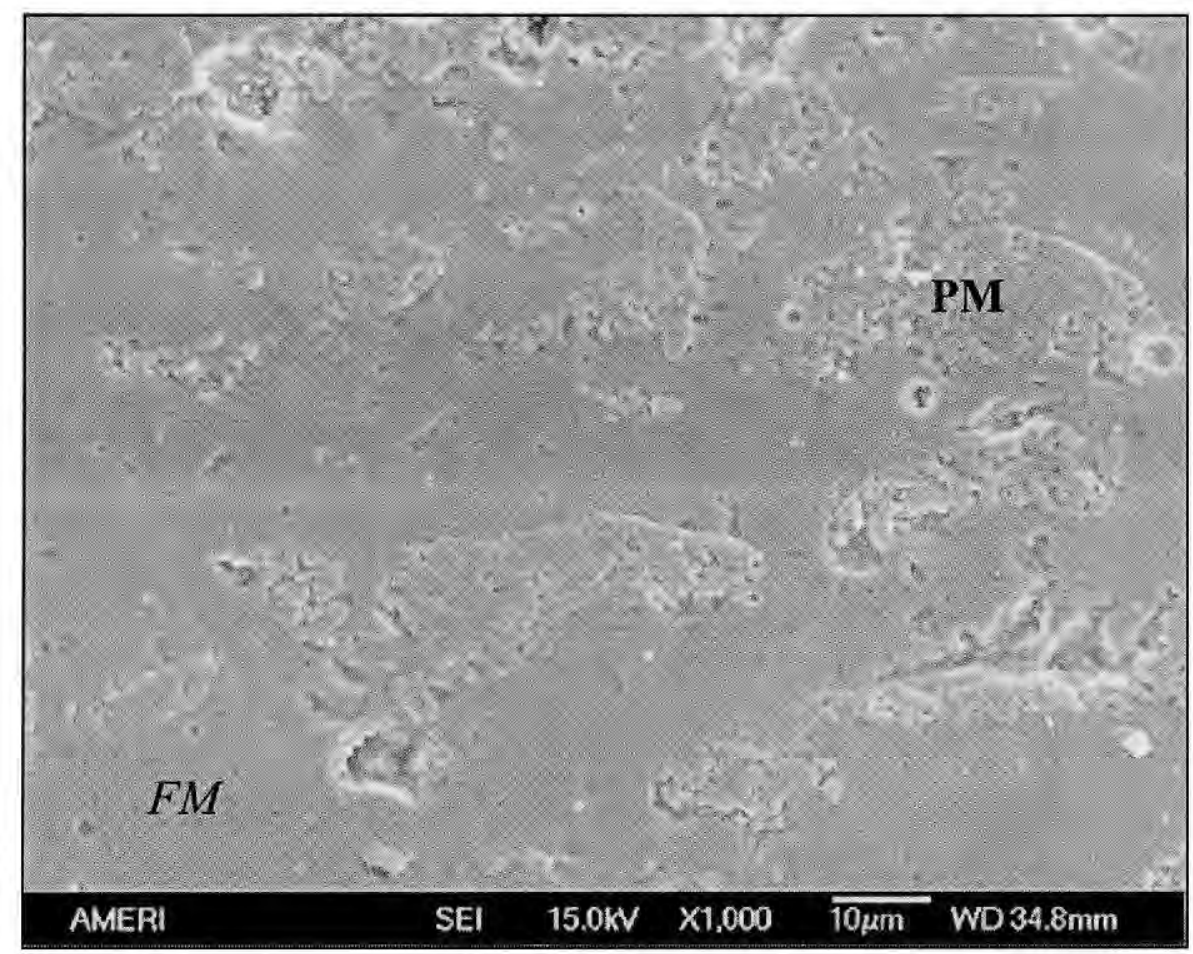

Fig. 4.18: Plasma sprayed A4C-B coating showing fully melted (FM) and solid state sintered (PM) region.

To preserve the advantages of CNT reinforcement, it is necessary for CNTs to survive their sojourn in the harsh environment of plasma plume. As seen in Fig. 4.19a and $4.19 \mathrm{~b}$, interlinked-CNTs are distributed, undamaged and retained in the plasma sprayed structure. Survival and distribution of CNTs is attributed to: i) dispersion of $\mathrm{CNTs}$ along the surface and the dome-cavity region of the spray dried $\mathrm{Al}_{2} \mathrm{O}_{3}$ powder 

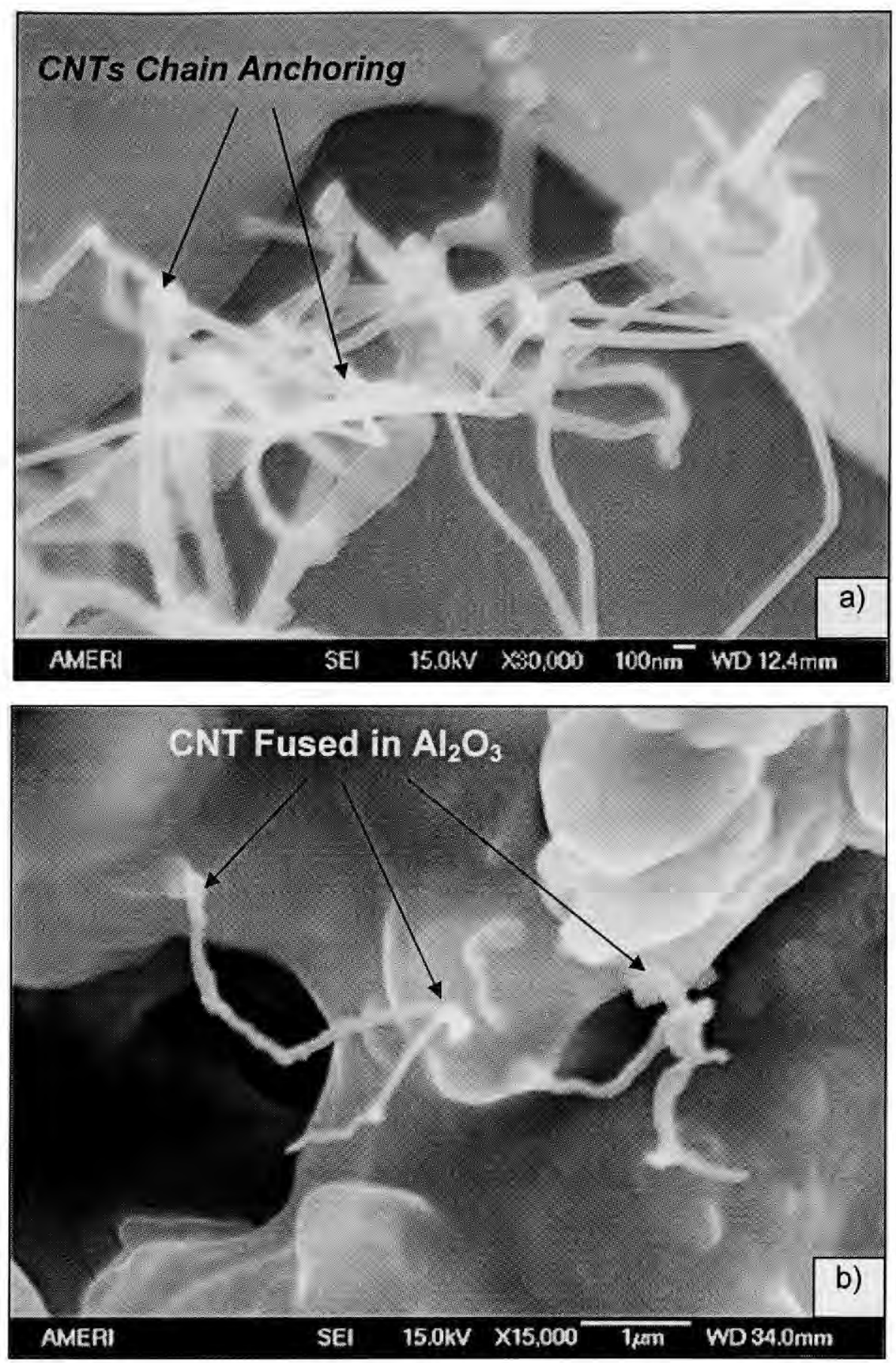

Fig. 4.19: Fractured surface image of A4C-B coating depicting a) chain loop formation of CNTs, and b) fusion of CNTs in $\mathrm{Al}_{2} \mathrm{O}_{3}$. 
(also seen in Fig. 4.3), and ii) controlled plasma processing parameters $(2898 \mathrm{~K}, 271$ $\mathrm{m} / \mathrm{s}$ ) to cause partial melting of core. Damage of CNTs from harsh plasma environment is also shrouded by poor thermal conductivity of powder-agglomerate porosity, and limited melting of the surface causing the core and tail regions of powder agglomerate to retain CNTs during successive deposition of splats. Survival of CNTs play an important role towards resulting microstructural ingenuity owing to their excellent mechanical properties [26, 31, 212]. CNT chain-loop formation, Fig. 4.19a, acts as anchors for providing improved fracture toughness. In addition, entangling of CNTs induce torsion, making torsion stiffness an important concept of providing toughness to the reinforced nanocomposite [213]. Fusion of CNTs along the $\mathrm{Al}_{2} \mathrm{O}_{3}$ melt, Fig. $4.19 \mathrm{~b}$, shows the good wettability observed in the plasma sprayed nanocomposite coating owing to high plasma parameters melting the $\mathrm{Al}_{2} \mathrm{O}_{3}$ surface and trapping the CNTs within.

\subsubsection{Plasma Sprayed A4C-SD Coating}

A uniform $\sim 450 \mu \mathrm{m}$ thick coating is obtained by plasma spraying A4C-SD powder, Fig. 4.20. Coating is dense (3.52 g/cc with $90.2 \%$ theoretical density) and adherent to the substrate. Higher degree of PM region is observed in the plasma sprayed A4C-SD coating, and is compared and quantified with other coatings in the later section. Processing of this coating is aimed to correlate the role of CNT dispersion in enhancing fracture toughening in comparison to $\mathrm{A} 4 \mathrm{C}-\mathrm{B}$ coating, which also has similar composition. 


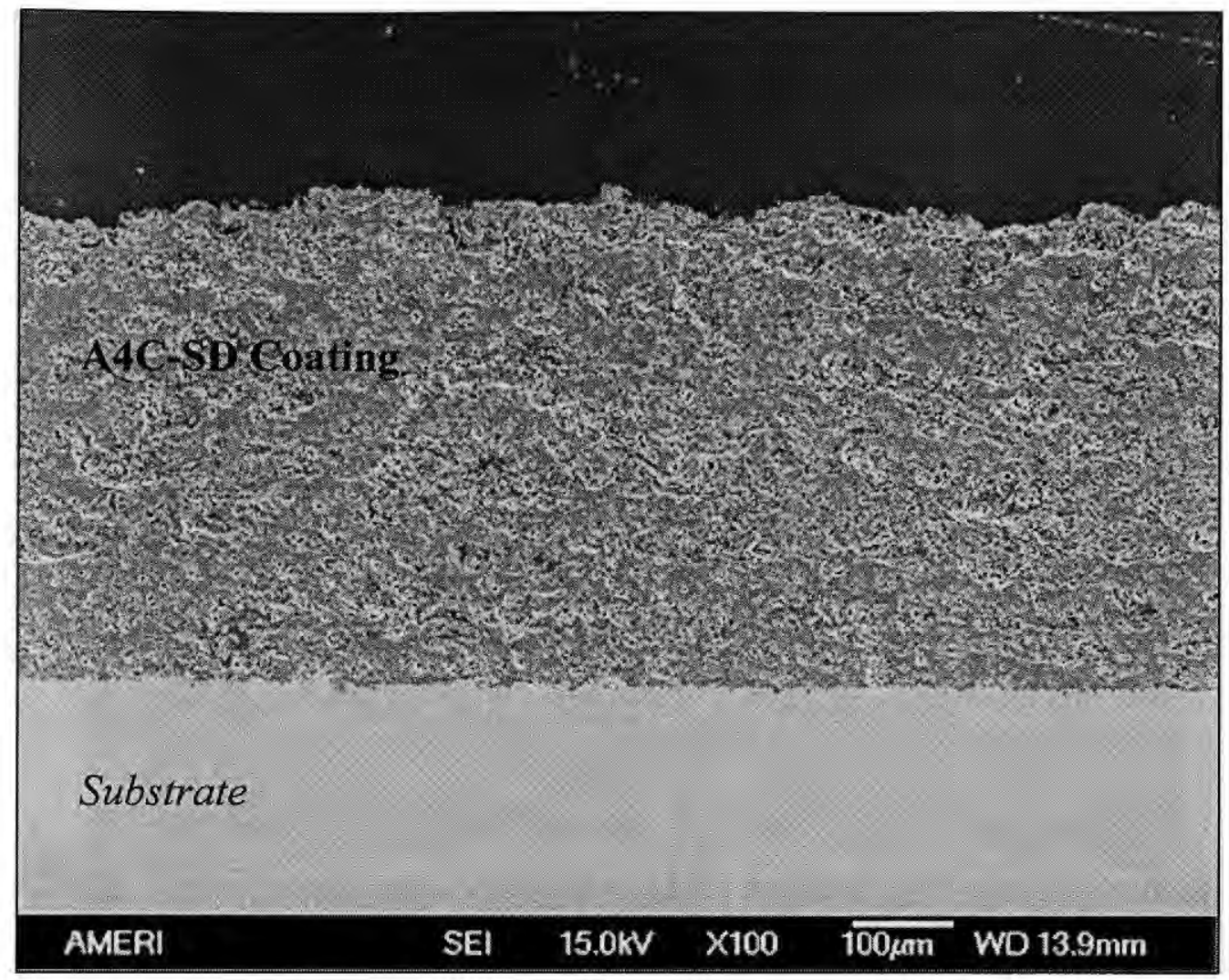

Fig. 4.20: Cross-section of plasma sprayed A4C-SD coating.

It is clearly indicated that the powder particles have undergone reduced temperature (of $2332 \mathrm{~K}$ when compared to $2898 \mathrm{~K}$ for A4C-B coating) deposition owing to its high PM content, Fig. 4.21. Inflight particle temperature data indeed confirms the role of CNT dispersion in reducing thermal exposure to the powders during plasma spraying.

CNTs are retained in the plasma sprayed A4C-SD coating, Fig. 4.22a. CNTs appear thicker $(\sim 100-120 \mathrm{~nm})$ when compared to starting CNTs $(40-70 \mathrm{~nm})$ implying that CNTs are coated with $\mathrm{Al}_{2} \mathrm{O}_{3}$ during processing. Spreading of CNTs throughout the matrix without agglomeration confirms good dispersion (Fig. 4.22b). 


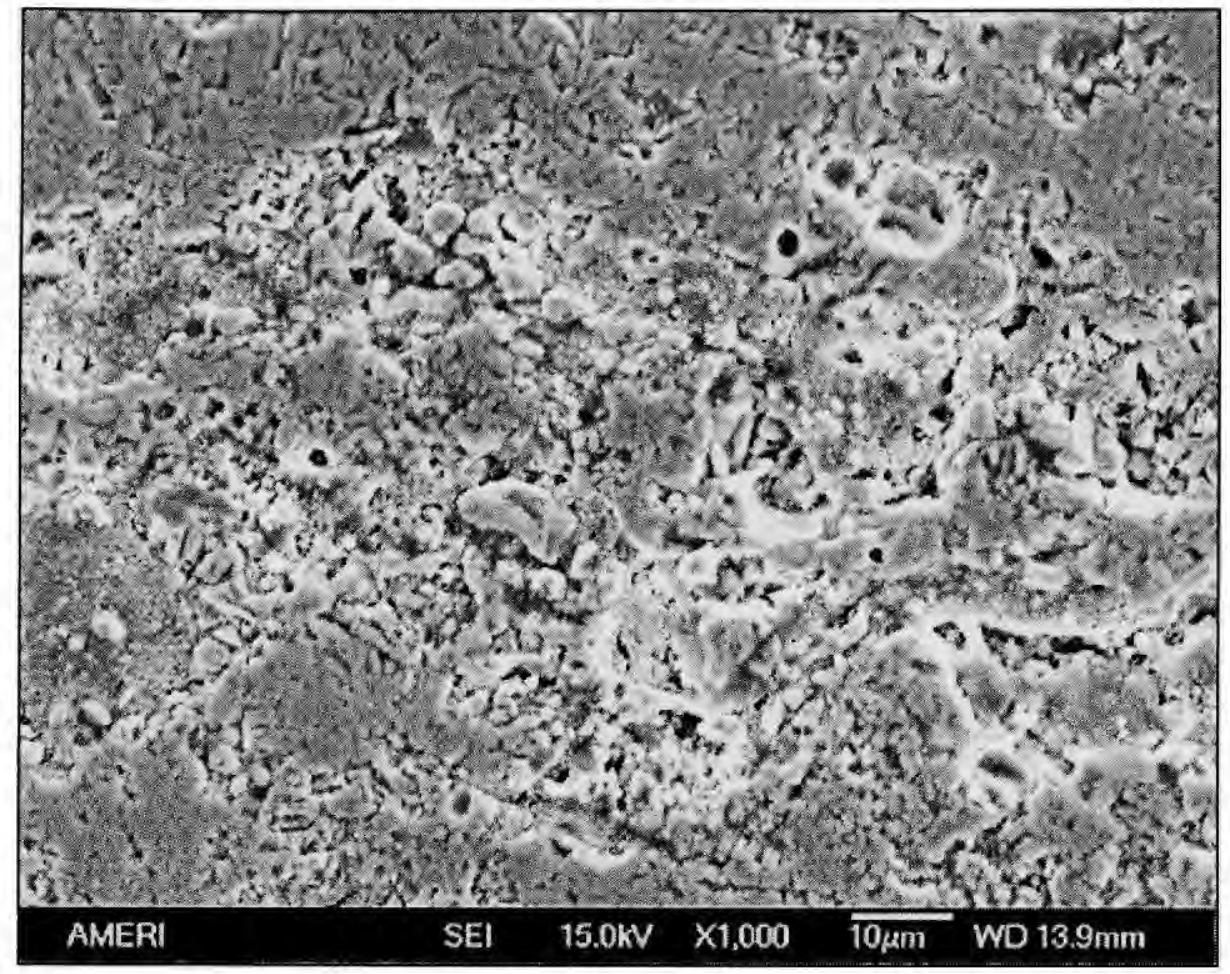

Fig. 4.21: Plasma sprayed A4C-SD coating depicting enhanced PM content.

CNT dispersion plays an important role in reducing thermal damage and consequently CNTs are retained in the matrix. CNTs are observed both near the PM and FM regions, indicating their survival in the plasma plume during their processing. Survival of CNTs in dispersed condition is critical towards uniform toughening of ceramic nanocomposite.

Enhanced wettability of CNTs is dictated by $\mathrm{Al}_{2} \mathrm{O}_{3}$ coating on CNT surface (indicated by increase in the CNT diameter), Fig. 4.22a, with good anchoring with the splats. On one hand where excellent wettability of CNTs anchors the splats, on the other hand, dispersed CNTs provide uniform reinforcements. Synergetic effect of dispersed CNTs depicting good wettability is expected to ensue enhanced fracture toughening of the A4C-SD coating. 

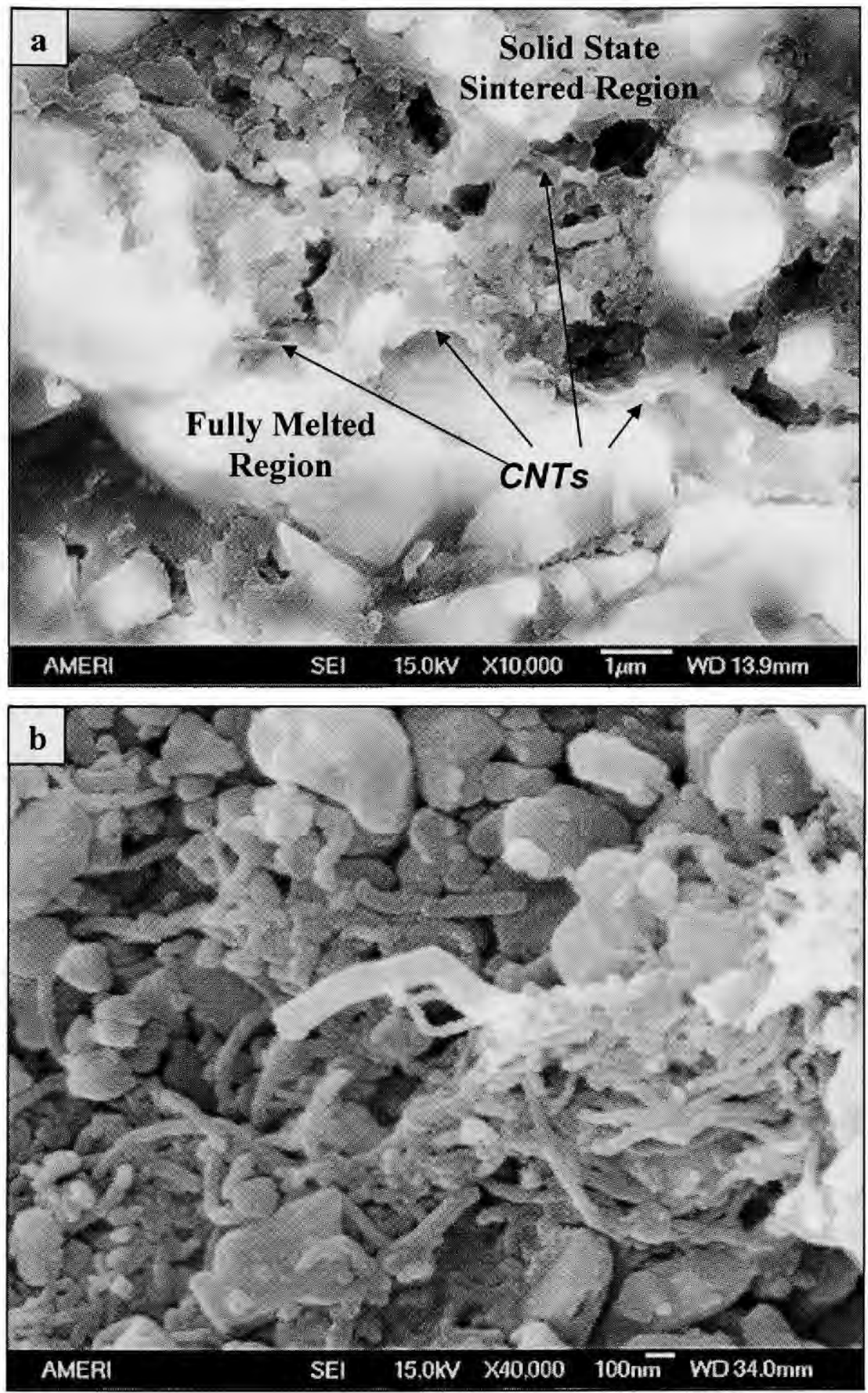

Fig. 4.22: a) Cross-section and b) fractured surface of plasma sprayed A4C-SD coating showing CNT retention and distribution. 
Most of the ceramic CNT composite processing is performed via solid state sintering techniques such as spark plasma sintering, hot pressing, extrusion, etc) consolidation $[16,172,176,186]$. Absence of molten ceramic in conventional processing (and in most of advanced techniques) has obviated attention to aspects of CNT surface coating and anchoring in such structures. Hence wetting of CNT by alumina is a novel issue by itself that has been discussed later.

\subsubsection{Plasma Sprayed A8C-SD Coating}

Plasma sprayed A8C-SD shows a uniform and $\sim 500 \mu \mathrm{m}$ thick coating in Fig. 4.23. Coating is dense $(3.53 \mathrm{~g} / \mathrm{cc}$ with $94 \%$ theoretical density) and adherent to the substrate. Magnified SEM image of the coating, Fig. 4.24, elicits similar features as observed for A4C-SD coating. Comparative solid state sintered region (PM), fully melted region (FM), and porosity is defined by the microstructural features, as discussed in section 4.5.1. Enhanced PM content is attributed to uniform CNT distribution and higher CNT content leading to reduced thermal exposure.

A typical solid state sintered region of A8C-SD coating is shown in Fig. 4.25a. Reduced thermal exposure $(2241 \mathrm{~K})$ to the powders is attributed to increased CNT content (from 4 wt. $\%$ to 8 wt.\%), which extracts heat from surrounding $\mathrm{Al}_{2} \mathrm{O}_{3}$ particles. Consequent consolidation, in turn, entraps dispersed CNTs in the solid state sintered region, Fig. 4.25a. CNT dispersion is also observed at neck region between two adjoining splats, resolidified region and surface of $\mathrm{Al}_{2} \mathrm{O}_{3}$ particles, Fig. 4.25b. CNTs remain undamaged and are uniformly dispersed at various locations such as melted and resolidified region, high-impact area of adjoining neck region and also at surface. 


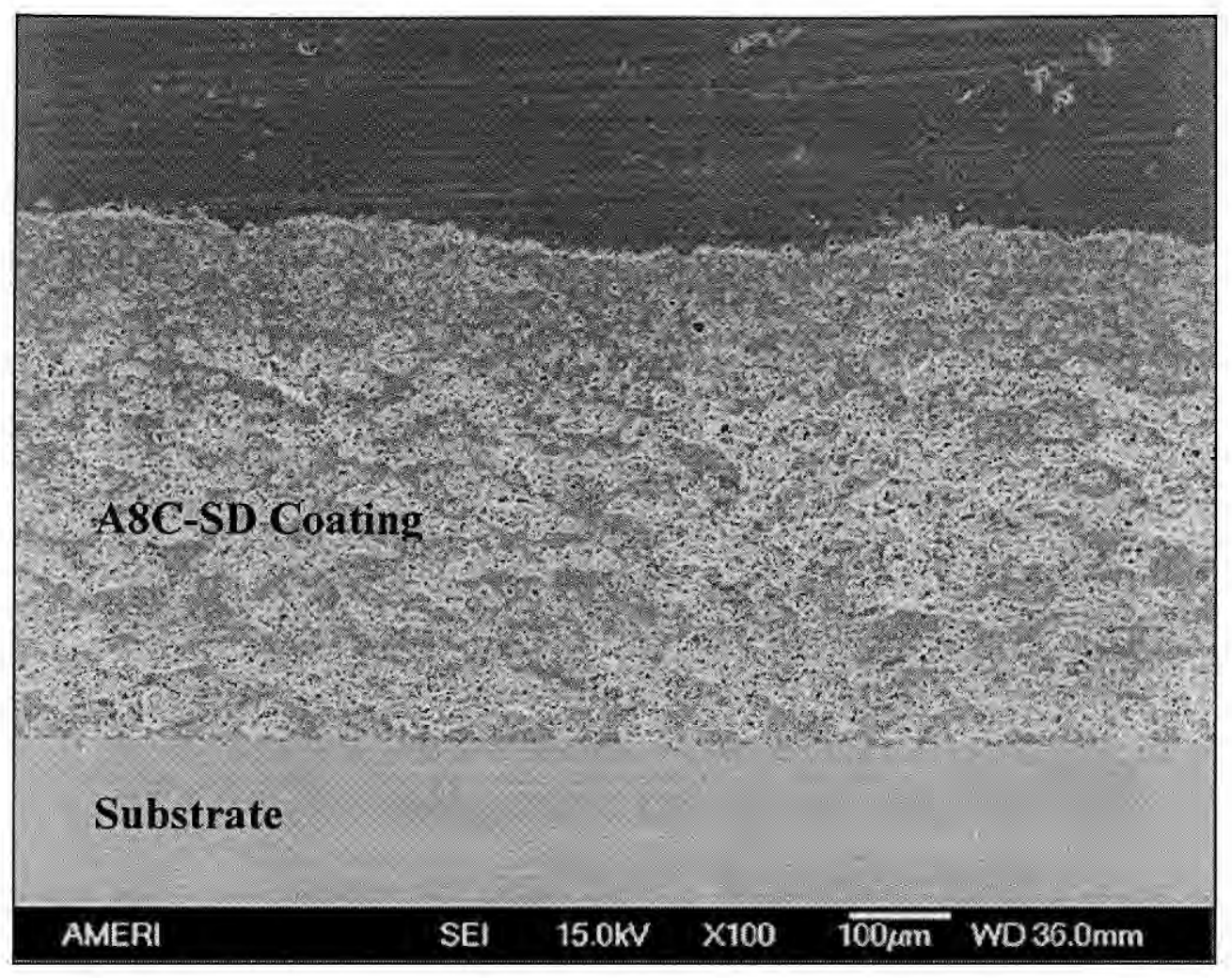

Fig. 4.23: Cross-sectional SEM image of plasma sprayed A8C-SD coating.

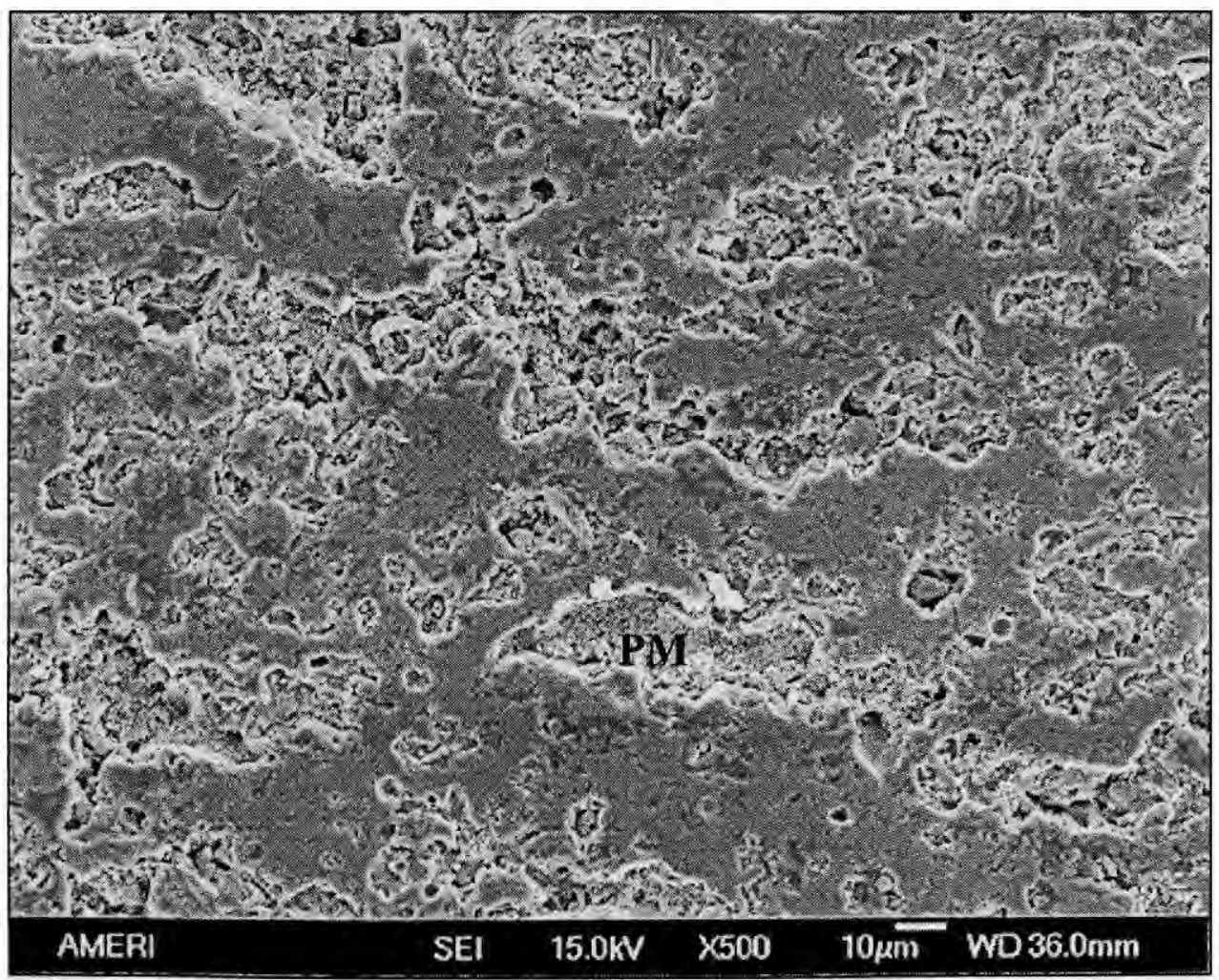

Fig. 4.24: Plasma sprayed A8C-SD coating showing FM and PM regions. 

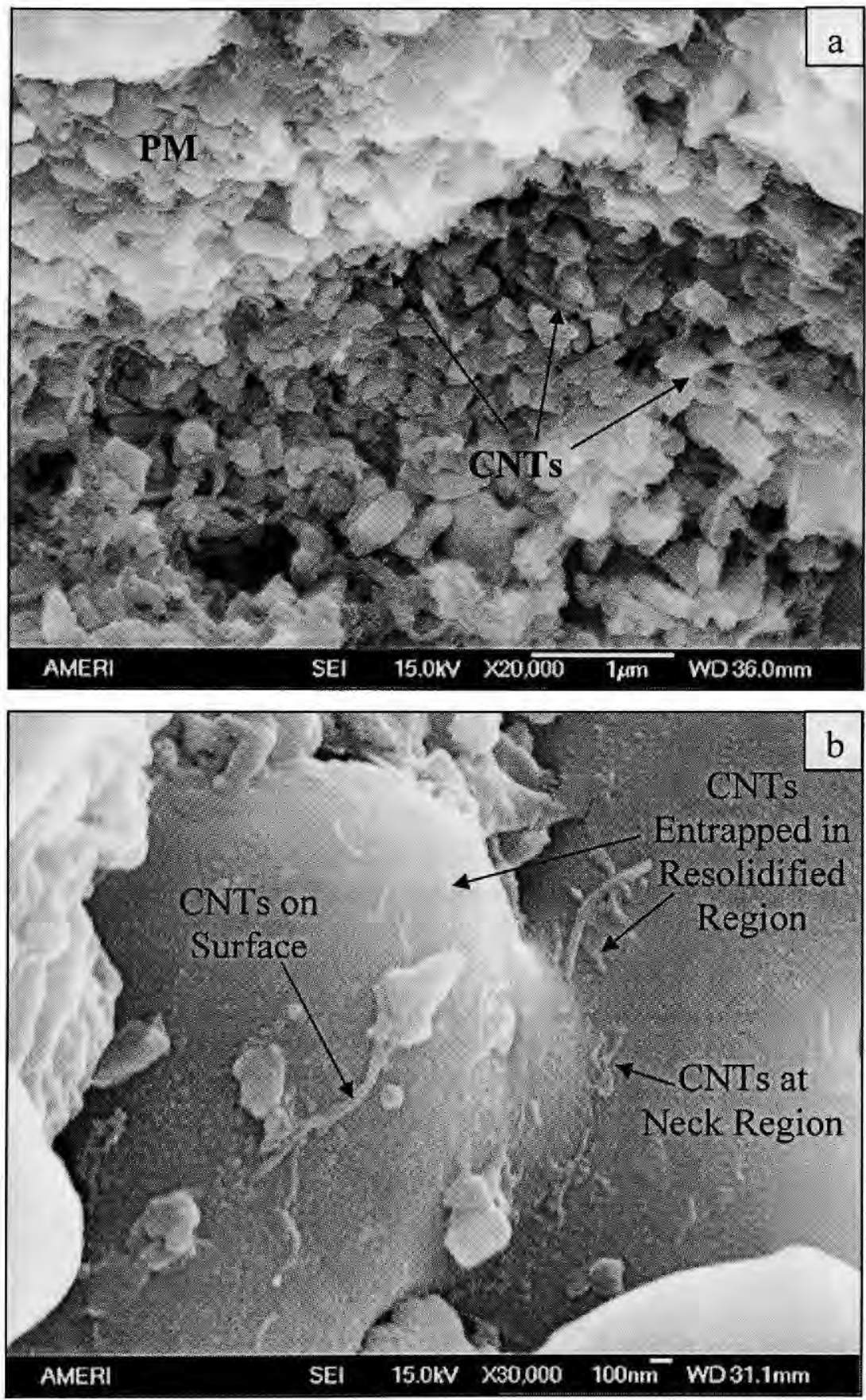

Fig. 4.25: Solid state sintered region showing a) CNT retention in the plasma sprayed A8C-SD coating, and b) CNT distribution at neck, resolidified region and surface of $\mathrm{Al}_{2} \mathrm{O}_{3}$ particles. 
Distribution of CNTs in the coatings is affirmed owing to retention of distributed $\mathrm{CNTs}$ as present in the starting A8C-SD powder.

The purpose of plasma spraying A8C-SD coating is to evaluate toughening properties with an increase in CNT content in comparison to A4C-SD coating. Hence it will be possible to capture fracture toughening enhancement both because of $C N T$ dispersion and CNT content.

Density of plasma sprayed coatings (measured from water immersion technique) is summarized in Fig. 4.26. Minimum density of $\sim 87 \%$ was achieved for A-SD coating, whereas addition of CNTs has resulted increased in the density of plasma sprayed coatings. This is attributed to dispersed CNTs promoting better heat transfer and thereby resulting enhanced densification. This is confirmed via enhanced PM region with

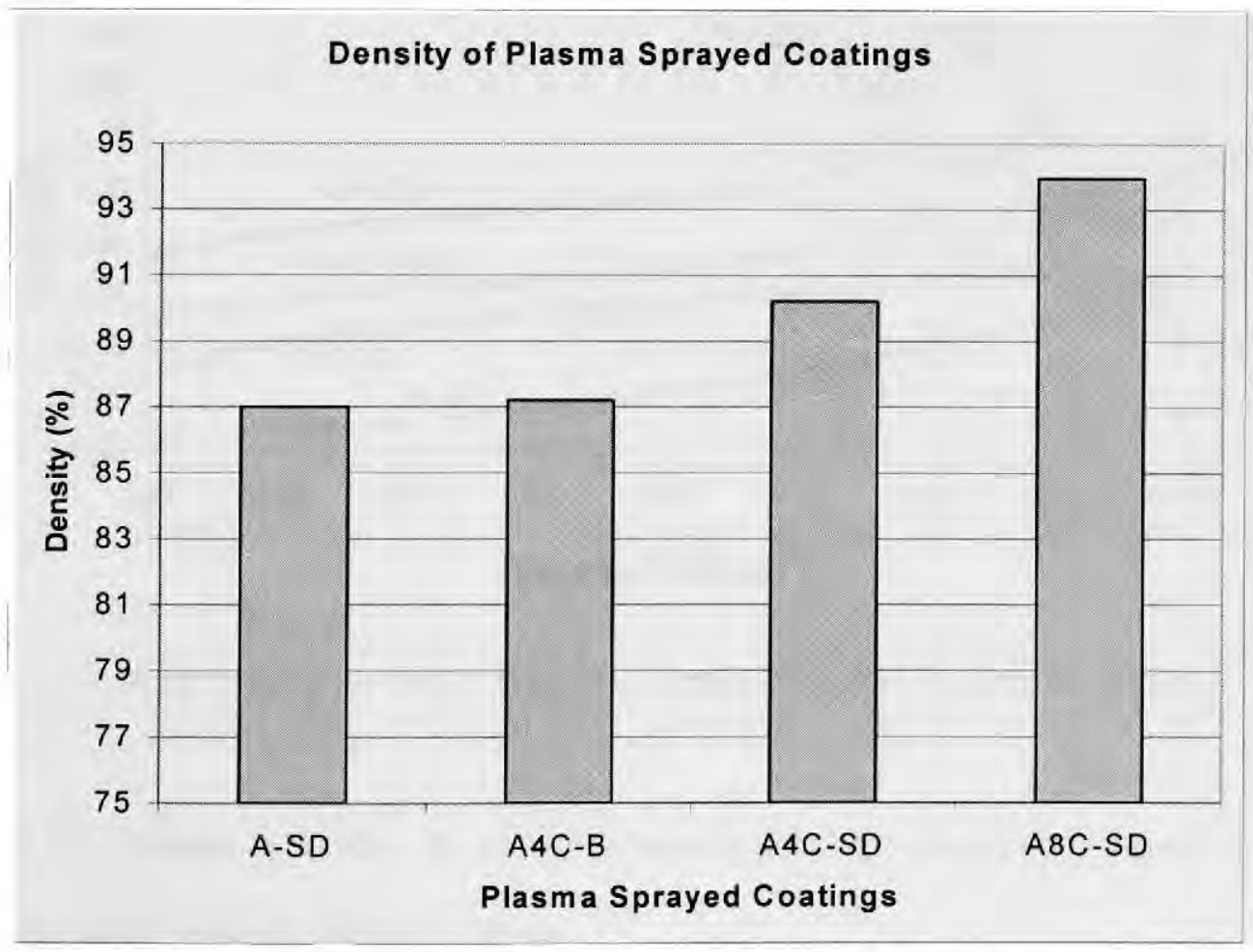

Fig. 4.26: Density of plasma sprayed coatings measured from water immersion technique. 
increase in CNT dispersion and CNT content. In addition, flexing nature of CNTs fill-in the gaps between $n-\mathrm{Al}_{2} \mathrm{O}_{3}$ splats and reduce the overall porosity during deposition.

\subsection{Retention of CNTs in Plasma Sprayed of $\mathrm{Al}_{2} \mathrm{O}_{3}$ - CNT Coatings}

Retention of CNT structure in the plasma sprayed coatings is confirmed by the D $\left(\sim 1340 \mathrm{~cm}^{-1}\right)$ and $\mathrm{G}$ peaks (between 1500 and $\left.1600 \mathrm{~cm}^{-1}\right)$, Fig. 4.27 which are same as observed in the initial powder feedstock. D-peak corresponds to disordered graphite, whereas G-peak corresponds to stretching mode of graphite, a characteristic of

\section{Raman Spectrum of Plasma Sprayed Coatings}

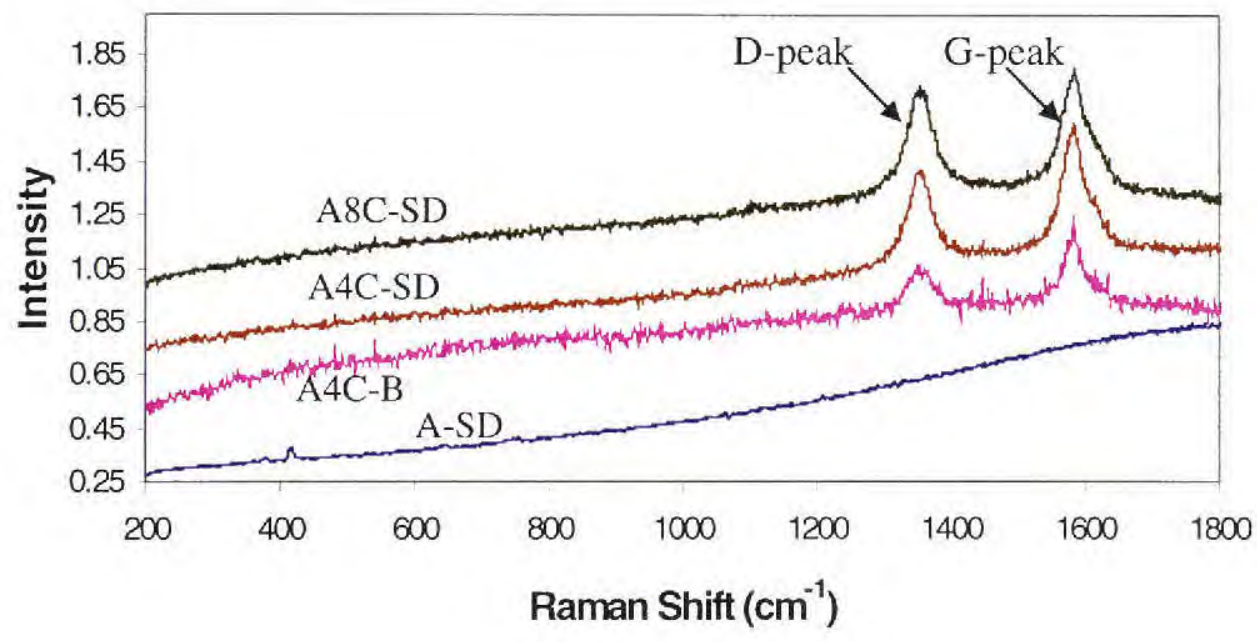

- ASD Coating - A4C-B Coating — AAC-SD Coating - ABC-SD Coating

Fig. 4.27: Raman spectrum of plasma sprayed coatings showing retention of CNT structure in the plasma sprayed coatings. 
nanotubes. Hence, Raman spectroscopy dictates that optimized plasma spraying parameters contribute in the retention of undamaged CNTs in the plasma sprayed coatings. This effect is assisted by enhanced porosity of spray-dried powder, which blankets the damage to CNTs via its reduced thermal conductivity, and adds in retaining CNTs even under high power plasma parameters.

\subsection{Effect of CNTs in Generating Microstructure and Phases in Plasma Sprayed Coatings}

Bimodal grain size distribution, corresponding inflight particle diagnostic data, generation of secondary metastable phases is presented in the current section. Microstructural PM and FM regions observed in the A-SD, A4C-B, A4C-SD, and A8CSD coatings are analyzed and influence of inflight particle diagnostic on generating such a microstructure is discussed. XRD analysis further elucidates the rapid kinetics inherent to the plasma spray processing.

\subsubsection{Role of CNT Dispersion in Creating Bimodal Grain Distribution in Matrix}

Distribution of the FM, PM and porosity in the plasma sprayed coatings is analyzed using ImagePro imaging software and presented in Table 4.7. Such a differential microstructure is obtained because of different thermal history experienced by powder particles during their transit through plasma plume.

Porosity measurement by quantitative microscopy considers only the provided image information, which in some cases might not be true representative. Different magnification images and selection of area for porosity calculation is user-dependent. 
Hence a more concrete approach of evaluating porosity by water immersion technique is utilized in the current research. Water immersion technique evaluates both macro and micro porosity of the plasma sprayed coatings, and, hence, is more reliable.

Table 4.7: Volume percentage of microstructural features of the plasma sprayed coatings obtained from quantitative microscopy and image analysis

\begin{tabular}{|c|c|c|c|c|}
\hline Coating & $\begin{array}{c}\text { Coating } \\
\text { Thickness }(\boldsymbol{\mu m})\end{array}$ & $\begin{array}{c}\text { Fully Melted } \\
(\mathbf{F M})\end{array}$ & $\begin{array}{c}\text { Partially Melted } \\
(\mathbf{P M})\end{array}$ & $\begin{array}{c}\text { Porosity } \\
\text { (Image } \\
\text { Analysis) }\end{array}$ \\
\hline A-SD Coating & 400 & $80.9 \%$ & $15.9 \%$ & $3.2 \%$ \\
\hline A4C-B Coating & 350 & $82.1 \%$ & $12.1 \%$ & $5.8 \%$ \\
\hline A4C-SD Coating & 450 & $71.4 \%$ & $24.7 \%$ & $3.9 \%$ \\
\hline A8C-SD Coating & 500 & $52.6 \%$ & $46.3 \%$ & $1.1 \%$ \\
\hline
\end{tabular}

Inflight particle diagnostic data was presented in Table 4.6. These contrasting velocities and temperature of inflight particles that form coatings indicate strong influence of CNT content and distribution in imparting reduced thermal exposure to the blended and spray dried powder particles. A4C-B coating experienced higher temperature when compared to A-SD coating, which is attributed to $\mathrm{CNT}$ distribution on the surface and the dome cavity (as explained in section 4.1). Owing to higher thermal conductivity of CNTs $(\sim 3000 \mathrm{~W} / \mathrm{mK})$ when compared to $\mathrm{Al}_{2} \mathrm{O}_{3}(\sim 36 \mathrm{~W} / \mathrm{mK})$, high heat is absorbed on the surface leading to enhanced thermal exposure and higher attained temperatures in A4C-B coating. Inflight particle diagnostic data, therefore affirms the mechanism of the heat distribution in the $\mathrm{A} 4 \mathrm{C}-\mathrm{B}$ coating. But, the reduction of thermal exposure in spray 
dried coatings (A4C-SD and A8C-SD coating) is attributed to uniform CNT dispersion both on the surface and inside of the powder particles (explained earlier in section 4.1). Directly stating, now less number of CNTs are present on the surface of spray dried powder (since content is same and CNTs are distributed uniformly also at inside of powder), thereby superheating of surface by CNTs is reduced. Additionally, CNT content in the inside of the powder now 'soaks up' the heat acquired by the surface CNTs towards reducing the temperature attained by powder particles. Hence, reduced temperature for A4C-SD coating was observed. Though addition of CNTs (in A8C-SD powder) marginally should increase the surface temperature, but uniform CNT distribution inside of the particle increases too, and the rate of soaking in the heat also increases. And owing to rapid kinetics inherent to the process, short residence times of $\sim$ $4 \times 10^{-4} \mathrm{~s}$ in the plasma plume do not suffice superheating of surface CNTs. This phenomenon further lowers the temperature acquired by the $\mathrm{A} 8 \mathrm{C}-\mathrm{SD}$ powder particles in the plasma plume. This offers two advantages: first that damage to CNTs is reduced due to reduced thermal exposure, and secondly that solid state sintered region can successfully retain CNTs.

It becomes clear now that increased temperature observed by $\mathrm{A} 4 \mathrm{C}-\mathrm{B}$ powder particles in plasma plume undergo higher degree of heating and induces higher FM content. Hence, it can be concluded that increasing plasma parameters and CNT content and dispersion are critical in defining temperature exposure experienced by powder particles and subsequent partially melted and fully melted structure. 


\subsubsection{Generation of Metastable $\gamma-\mathrm{Al}_{2} \underline{\mathrm{O}}_{3}$}

Owing to rapid kinetics of the plasma spraying (with cooling rates as high as 7.85 $x 10^{6} \mathrm{~K} / \mathrm{s}$ ), generation of metastable phases becomes inherent. XRD spectrum of the coatings is presented in Fig. 4.28. Since CNT peaks are not observed in the presence of $\mathrm{Al}_{2} \mathrm{O}_{3}$, only one XRD spectrum for powder feedstock is shown (instead of individual A$\mathrm{SD}, \mathrm{A} 4 \mathrm{C}-\mathrm{B}, \mathrm{A} 4 \mathrm{C}-\mathrm{SD}$, and A8C-SD powder spectra). Scherrer formula is used to calculate the crystallite size of the phases $(t)$ present in powder feedstock and plasma sprayed coatings.

$$
t=\frac{0.9 \lambda}{B \cos \theta} \quad \text { Equation } 4.1
$$

where $\lambda$ is the $\mathrm{X}$-ray wavelength, $\theta$ is the diffraction angle, $\mathrm{B}$ is the peak broadening measured at half of maximum intensity in radians (obtained as $\sqrt{B_{M}^{2}-B_{S}^{2}}$ where $B_{M}$ is the measured broadening at peak intensity half maximum, and $B_{S}$ is the peak intensity half-maximum of the standard). Silicon (Si) from NIST is taken as standard for calculating peak broadening of machine.

For crystallite size measurement of $\alpha-\mathrm{Al}_{2} \mathrm{O}_{3}, \mathrm{FWHM}$ (full width at half maximum) at most intense peak of 2-theta ( 35 degrees) is considered, whereas for $\gamma$ $\mathrm{Al}_{2} \mathrm{O}_{3}, \mathrm{FWHM}$ (full width at half maximum) at most intense peak of 2-theta $(\sim 46$ degrees) is measured. Alpha and gamma $\mathrm{Al}_{2} \mathrm{O}_{3}$ content was measured with the ratio of area of corresponding peaks to the area under cumulative peaks. It must be noted that starting powder feedstock was $100 \% \alpha-\mathrm{Al}_{2} \mathrm{O}_{3}$, hence only the crystallite size is reported in Table 4.8 . 


\section{XRD Spectrum of Plasma Sprayed Coatings}

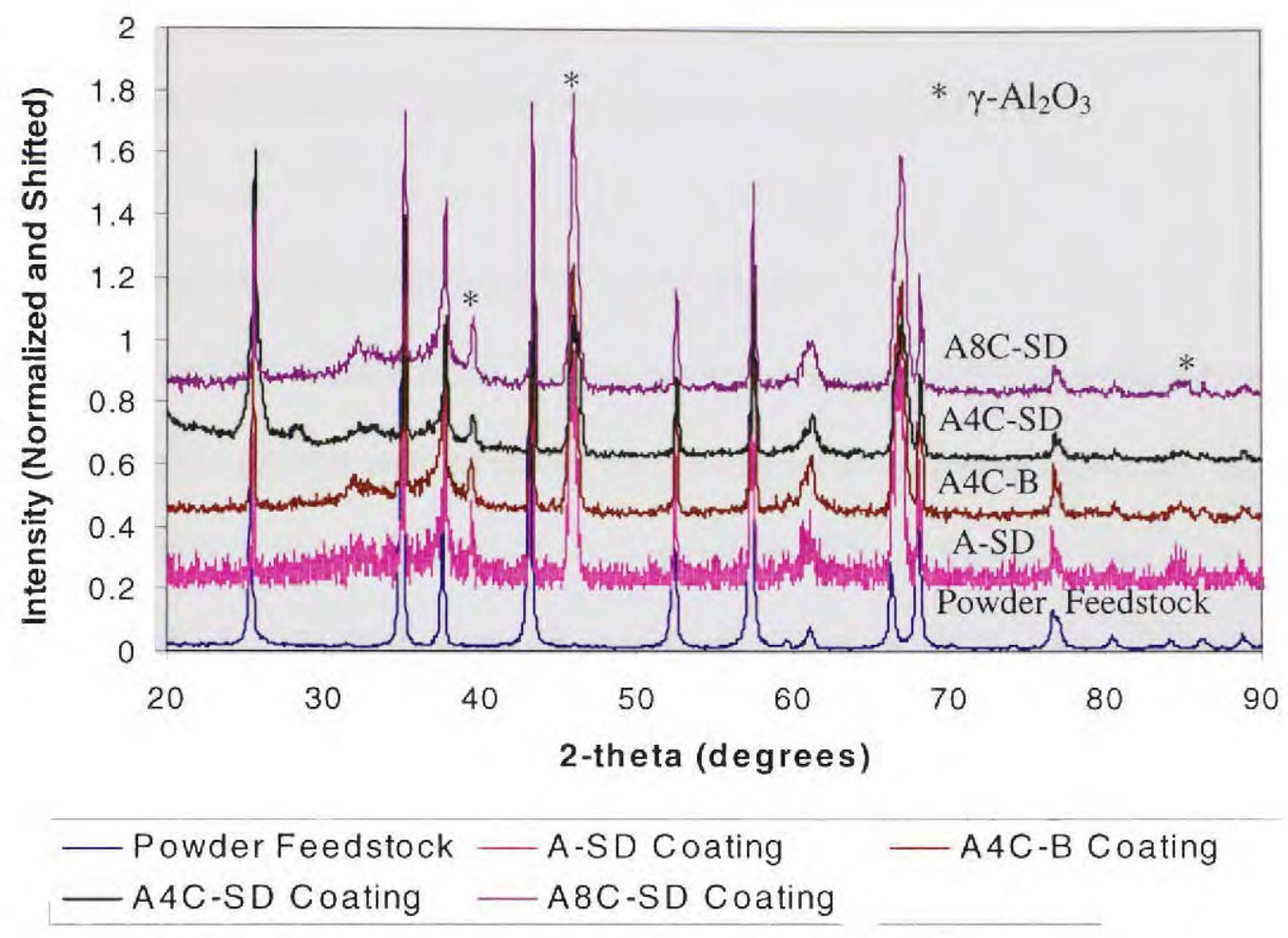

Fig. 4.28: XRD spectrum of plasma sprayed coatings. Indicated peaks belong to $\gamma-\mathrm{Al}_{2} \mathrm{O}_{3}$ whereas all other peaks belong to $\alpha-\mathrm{Al}_{2} \mathrm{O}_{3}$.

Table 4.8: Crystallite size and content of powder and plasma sprayed coatings

\begin{tabular}{|c|c|c|c|c|c|}
\hline \multirow{3}{*}{$\begin{array}{l}\text { Crystallite Size } \\
\text { and Phase } \\
\text { Content }\end{array}$} & \multirow{3}{*}{$\begin{array}{c}\text { Powder* } \\
\begin{array}{c}\text { Size } \\
(\mathrm{nm})\end{array}\end{array}$} & \multicolumn{4}{|c|}{ Coating } \\
\hline & & \multicolumn{2}{|c|}{$\alpha-\mathrm{Al}_{2} \mathrm{O}_{3}$} & \multicolumn{2}{|c|}{$\gamma-\mathrm{Al}_{2} \mathrm{O}_{3}$} \\
\hline & & $\begin{array}{l}\text { Size } \\
(\mathrm{nm})\end{array}$ & $\begin{array}{c}\text { Content } \\
(\%)\end{array}$ & $\begin{array}{l}\text { Size } \\
(\mathrm{nm})\end{array}$ & $\begin{array}{c}\text { Content } \\
\text { (\%) }\end{array}$ \\
\hline A-SD & 44 & 55 & 76.6 & 22 & 23.4 \\
\hline A4C-B & 46 & 95 & 68.4 & 13 & 31.6 \\
\hline $\mathrm{A} 4 \mathrm{C}-\mathrm{SD}$ & 45 & 73 & 81.7 & 15 & 18.3 \\
\hline A8C-SD & 43 & 76 & 74.9 & 21 & 25.1 \\
\hline
\end{tabular}

*Powder is $100 \% \alpha-\mathrm{Al}_{2} \mathrm{O}_{3}$. 
Presence of CNTs affects the nucleation of $\gamma-\mathrm{Al}_{2} \mathrm{O}_{3}$ that occurs from the melting and rapid solidification of $\alpha-\mathrm{Al}_{2} \mathrm{O}_{3}$. High thermal conductivity and superheating of CNTs create favorable sites for nucleation of $\gamma-\mathrm{Al}_{2} \mathrm{O}_{3}$. Generation of $\gamma-\mathrm{Al}_{2} \mathrm{O}_{3}$ phase is related to the CNT dispersion and location in the powder agglomerate. Nucleation of $\gamma-\mathrm{Al}_{2} \mathrm{O}_{3}$ phase is dependent on the content, location and dispersion behavior of CNTs.

\subsubsection{Dependence of $y-\mathrm{Al}_{2} \mathrm{O}_{3}$ on CNTs in the Matrix}

CNTs on the surface of the powder particle are responsible for nucleating $\gamma-\mathrm{Al}_{2} \mathrm{O}_{3}$ by absorbing the plasma heat, assisting the melting on surrounding alpha, and leading to enhanced cooling rates in its vicinity to generate $\gamma-\mathrm{Al}_{2} \mathrm{O}_{3}$. Owing to their high thermal conductivity $\left(\sim 3 \times 10^{3} \mathrm{~W} / \mathrm{m} / \mathrm{K}\right)$ when compared to that of $\mathrm{Al}_{2} \mathrm{O}_{3}(\sim 36 \mathrm{~W} / \mathrm{m} / \mathrm{K})$, CNTs heat up melting the surrounding $\mathrm{Al}_{2} \mathrm{O}_{3}$, and quickly lose heat to cooler surrounding $\mathrm{Al}_{2} \mathrm{O}_{3}$ in the core (because of optimized surface meting and solid state sintering of core). Rapid kinetics involved inherently with the processing adds to the $\gamma-\mathrm{Al}_{2} \mathrm{O}_{3}$ nucleation in the processed coating. Hence surface CNTs are responsible for enhancing the gamma content of the plasma spayed structure.

CNTs entrapped in the core absorb the heat from the surface. Since the travel time of powder particles is in order of $\sim 4 \times 10^{-4} \mathrm{~s}$, CNTs present in the core do not allow superheating of the CNTs located on the surface. Restriction to reach high temperature presents two benefits in the processed coatings. First it shrouds the damage to surface CNTs by restricting the CNT temperatures. This behavior is linked by the inflight particle diagnostic data observed for the spray dried coatings, where reduced temperature profiles

were observed even for higher plasma parameters (Table 4.7). Secondly, it reduces the 
gamma precipitation on CNTs in core by disallowing high cooling rates as experienced by surface CNTs in the absence of core CNTs.

Dome cavity CNT agglomeration (as in A4C-B coating) can achieve exceedingly high temperatures owing to agglomerated mass of CNTs. Reduced resistance path and high thermal conductivity of CNTs renders high cooling rates to the surrounding $\mathrm{Al}_{2} \mathrm{O}_{3}$. Absence of core-CNTs not only tender comparatively enhanced damage to surfaceCNTs, but also is expected to impart enhanced $\gamma-\mathrm{Al}_{2} \mathrm{O}_{3}$ precipitation. Though $\gamma-\mathrm{Al}_{2} \mathrm{O}_{3}$ assists with second phase strengthening, the total potential of CNT-toughening goes untapped.

Dispersion of CNTs (as in A4C-SD and A8C-SD coatings) allows entrapment of CNTs both in the molten- and solid state sintered region. CNTs present in the core region are retained by solid state sintering and retain entrapped in the densified region. Surface CNTs disperse along intersplat region as successive impact of molten/semi-molten particles. Therefore, dispersion of CNTs potentially creates more sites for $\gamma-\mathrm{Al}_{2} \mathrm{O}_{3}$ nucleation. But two factors must be considered before making conclusions. First that since CNTs act as nucleating sites for $\gamma-\mathrm{Al}_{2} \mathrm{O}_{3}$, increasing CNT content overall increases the $\gamma-\mathrm{Al}_{2} \mathrm{O}_{3}$ content. Secondly that CNTs in the core act as 'heat absorbing' sites to reduce the surface temperature of the $\mathrm{Al}_{2} \mathrm{O}_{3}$, thereby reducing the degree of melting and kinetics of rapid solidification. Hence precipitation of $\gamma-\mathrm{Al}_{2} \mathrm{O}_{3}$ is a balance between CNT dispersion, surface distribution and total CNT content.

It can be reasoned from the observed date, Table 4.8, that addition of CNTs increased gamma content, but reduces size of gamma crystallite (A4C-B coating when compared to A-SD coating). This behavior is attributed to distribution of CNTs only on 
the surface. Since CNTs are distributed onto the surface, rapid quenching due to thermal heat absorption enhances the kinetics increases the nucleation rate, but reduces the growth rate, thereby reduce the $\gamma-\mathrm{Al}_{2} \mathrm{O}_{3}$ size and increase the $\gamma-\mathrm{Al}_{2} \mathrm{O}_{3}$ content.

Contrasting feature of CNT dispersion is observed in the spray dried powder, where reduced $\gamma-\mathrm{Al}_{2} \mathrm{O}_{3}$ size and content is observed (A4C-SD coating compared to A-SD coating). Since CNTs are dispersed throughout the powder, superheating of CNTs is restricted by CNTs present in the core of the powder agglomerate. On one hand, the overall content of CNTs on the surface of A4C-SD powder is less (when compared to A4C-B powder because of same CNT content with better dispersion even in the core of powder), and secondly core CNTs soak up the thermal heat of the surface CNTs. Hence overall sites for gamma nucleation are reduced and enhanced time for cooling (reduced cooling rate) allows only minimal precipitation of $\gamma-\mathrm{Al}_{2} \mathrm{O}_{3}$.

Now, with the A8C-SD powder, interesting feature of both enhanced $\gamma-\mathrm{Al}_{2} \mathrm{O}_{3}$ size and content (when compared to A4C-SD powder) is observed. This is attributed to similar dispersion as that of A4C-SD powder, but enhanced CNT content on the surface now leads to more nucleating sites (and increased $\gamma-\mathrm{Al}_{2} \mathrm{O}_{3}$ content). In addition to reduced cooling rate (via increased heat absorption by core CNTs), and enhanced chances of merging the precipitated $\gamma-\mathrm{Al}_{2} \mathrm{O}_{3}$ crystallites by amplified CNT content shows increased $\gamma-\mathrm{Al}_{2} \mathrm{O}_{3}$ size.

Generation of metastable phases is inherent in the plasma sprayed structures. Considering the high energy input of plasma process, the free energy change associated with $\alpha-\mathrm{Al}_{2} \mathrm{O}_{3}$ to $\gamma-\mathrm{Al}_{2} \mathrm{O}_{3}$ phase transformation can be described as [[214]]: 


$$
\Delta G_{r}=\Delta G_{v o l}+\Delta \Gamma_{\text {surface }}=\frac{4}{3} \pi r^{3}\left(G_{\gamma}-G_{\alpha}\right)+4 \pi r^{2}\left(\Gamma_{\gamma}-\Gamma_{\alpha}\right) \quad \text { Equation } 4.2
$$

where $\mathrm{G}_{\alpha}$ and $\mathrm{G}_{\gamma}$ are free energies, and $\Gamma_{\alpha}$ and $\Gamma_{\gamma}$ are surface energies of $\alpha-\mathrm{Al}_{2} \mathrm{O}_{3}$ and $\gamma$ $\mathrm{Al}_{2} \mathrm{O}_{3}$ respectively of radius $\mathrm{r}$.

Since $\gamma-\mathrm{Al}_{2} \mathrm{O}_{3}$ is a metastable phase, it has critical radius of transformation much smaller than existing $\alpha-\mathrm{Al}_{2} \mathrm{O}_{3}$ phase. Considering the crystallite size of $\alpha-\mathrm{Al}_{2} \mathrm{O}_{3} \rightarrow \gamma$ $\mathrm{Al}_{2} \mathrm{O}_{3}$ phase transformation, the critical radius $r_{c}$ is obtained by equating the differential, with respect to $\mathrm{r}$, of eq. 4.2 to zero.

$$
r_{c}=\frac{-2\left(\Gamma_{\gamma}-\Gamma_{\alpha}\right)}{\left(G_{\gamma}-G_{\alpha}\right)}
$$

Equation 4.3

Though the $\gamma-\mathrm{Al}_{2} \mathrm{O}_{3}$ crystallites will grow as coarsening progresses, they will serve as second phase particles and help improving the dispersion strengthening of the composite. Hence, CNTs become critical in manipulating the bimodal phase distribution in the plasma sprayed coatings and take advantage of secondary strengthening mechanisms.

High magnification TEM image of CNT surface, Fig. 4.29a, shows precipitation of $\gamma-\mathrm{Al}_{2} \mathrm{O}_{3}$ crystallites. High resolution TEM imaging, Figs. $4.29 \mathrm{~b}$ and $\mathrm{c}$, show lattice fringes of the generated precipitate. Lattice spacing of fine crystallite corresponds to 2.19 $\AA$, which matches with the interplanar crystal spacing of (023) plane for $\gamma-\mathrm{Al}_{2} \mathrm{O}_{3}$ phase. Presence of fine $\gamma-\mathrm{Al}_{2} \mathrm{O}_{3}$ precipitates on CNT surface confirms the proposed mechanism of $\gamma-\mathrm{Al}_{2} \mathrm{O}_{3}$ phase generation. 

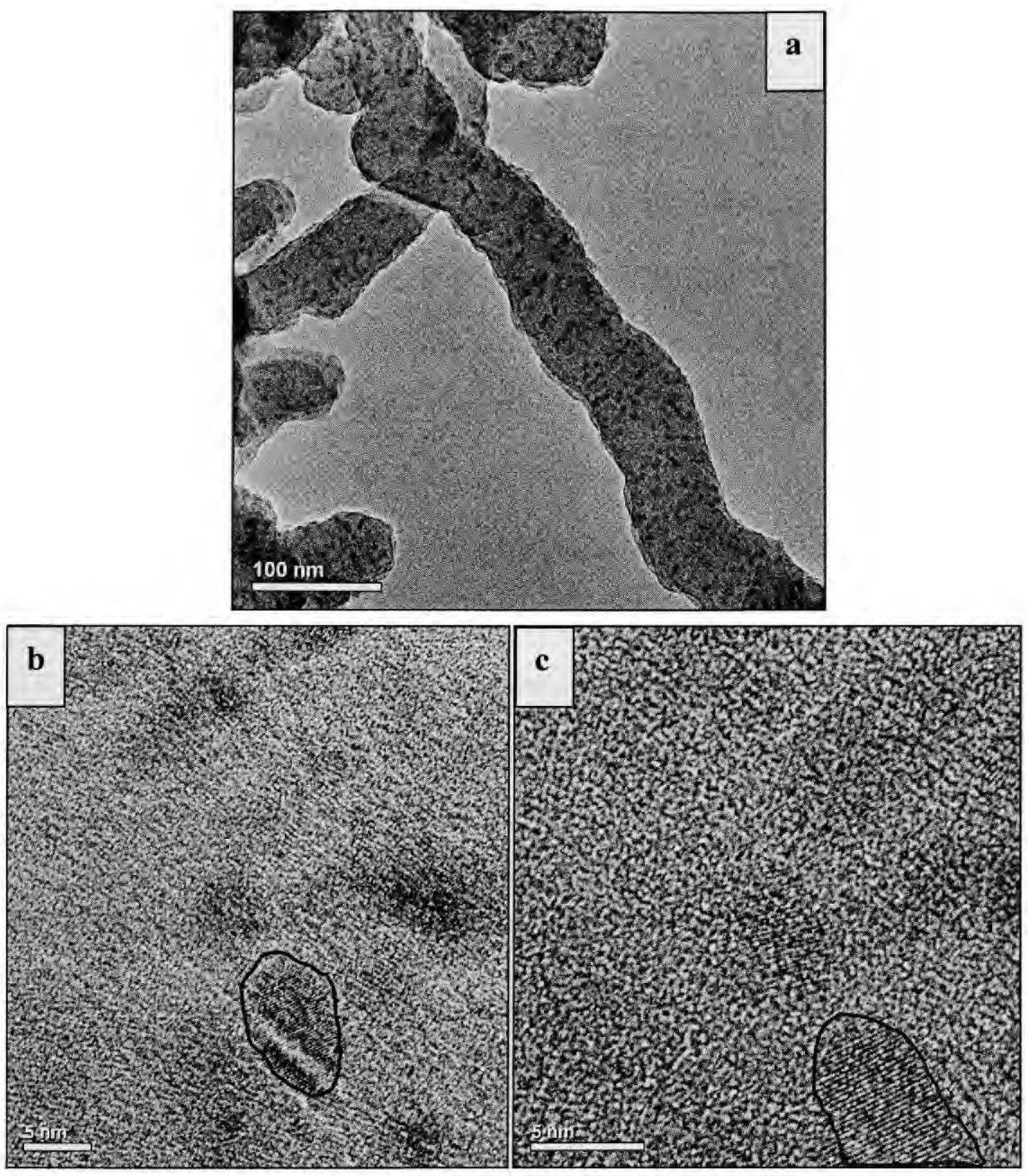

Fig. 4.29; TEM image showing a) $\gamma-\mathrm{Al}_{2} \mathrm{O}_{3}$ precipitation on CNT surface, and High resolution TEM image showing $\gamma-\mathrm{Al}_{2} \mathrm{O}_{3}$ lattice fringes corresponding to b) (311) plane (lattice spacing of $2.38 \AA$, and c) (023) plane (lattice spacing of $2.19 \AA$ ). 


\subsection{Role of CNT Dispersion in the Fracture Toughening of Nanocomposite Ceramic Coatings}

Role of CNT dispersion is measured by an increase in the fracture toughness of the plasma sprayed coatings. Measure of toughening by CNT pull out, crack bridging, and crack deflection is reflected in the high magnification SEM microscopy of the coatings. Vicker indentation is utilized for measuring indentation toughness with a load of $200 \mathrm{~g}$ with a dwell time of $15 \mathrm{~s}$.

Table 4.9 details the hardness and fracture toughness of the plasma sprayed coatings. It can be noted that A4C-B there is no significant variation in the hardness value of the different coatings. Though it might be expected that higher density of coatings result higher hardness, but it is counter balanced by the reduction of hardness due to increasing PM content. Hence, overall hardness of the plasma sprayed coatings show similar hardness values in Vicker indentation.

Table 4.9: Hardness and fracture toughness of plasma sprayed coatings

\begin{tabular}{|c|c|c|c|c|}
\hline Coating & A-SD & A4C-B & A4C-SD & A8C-SD \\
\hline $\begin{array}{c}\text { Density g/cc } \\
(\% \text { theoretical) }\end{array}$ & $3.47(87 \%)$ & $3.40(87.2 \%)$ & $3.52(90.2 \%)$ & $3.53(94 \%)$ \\
\hline Hardness $\left(\mathrm{V}_{\mathrm{H}}\right)$ & $714.2 \pm 14.9$ & $709.6 \pm 8.4$ & $738.7 \pm 12.9$ & $712.7 \pm 2.3$ \\
\hline $\begin{array}{c}\text { Fracture Toughness } \\
\left(\mathrm{MPa} \mathrm{m}{ }^{1 / 2}\right)\end{array}$ & $3.22 \pm 0.22$ & $3.86 \pm 0.16$ & $4.60 \pm 0.27$ & $5.04 \pm 0.58$ \\
\hline
\end{tabular}

Fracture toughness $\left(K_{c}\right)$ of the ceramic coatings, Fig. 4.29 , is calculated from Antis Equation as [215]: 


$$
K_{c}=\chi\left(\frac{E}{H}\right)^{1 / 2} \frac{P}{c^{3 / 2}}
$$

where $P$ is the applied load, $E$ is the Young's modulus (taken as $390 \mathrm{GPa}$ ), $H$ is the Vickers hardness, $c$ is the radial crack length (measured from center of indent), and $\chi$ is the calibration constant (taken as 0.016 ).

Vicker indentation fracture toughness of coatings is calculated as $3.22 \mathrm{MPa} \mathrm{m}$ for A-SD coating, whereas it increases to $3.86 \mathrm{MPa} \mathrm{m}^{1 / 2}$ for $\mathrm{A} 4 \mathrm{C}-\mathrm{B}$ coating, an improvement of $20 \%$, Fig. 4.30. Enhancement of fracture toughness is solely arising from the introduction of $4 \mathrm{wt} . \% \mathrm{CNT}$ in the matrix. But, A4C-SD coating shows fracture toughness of $4.60 \mathrm{MPa} \mathrm{m}^{1 / 2}$ (Fig. 4.30), an improvement of $43 \%$ over A-SD coating. Though CNT contents are same in A4C-B and A4C-SD coating, tremendous improvement in the fracture toughness is attributed to uniform CNT dispersion in the A4C-SD coating when compared to A4C-B coating. Further increase in fracture toughness $\left(5.04 \mathrm{MPa} \mathrm{m}^{1 / 2}\right)$ to $56 \%$ is achieved for A8C-SD coating, which is attributed to increase in the CNT content. Student t-test was performed to confirm significant statistical difference between the mean fracture toughness values with more than $95 \%$ confidence level.

It must be noted that porosity content, porosity size and porosity distribution can also alter the fracture toughness of the nanocomposites $[10,45,56]$. The dispersion of CNTs (in A4C-B, A4C-SD and A8C-SD) has shown contrasting change in the thermal exposure to inflight powders (Table 4.6), which consequently results in the variation in porosity in the plasma sprayed coatings, as seen in Fig. 4.26. Table 4.5 shows that higher plasma power of $30.8 \mathrm{~kW}$ for $\mathrm{A} 4 \mathrm{C}-\mathrm{SD}$ and $32.3 \mathrm{~kW}$ for $\mathrm{A} 8 \mathrm{C}-\mathrm{SD}$ was used when 
compared to A4C-B powders (power of $25.7 \mathrm{~kW}$ ). Higher plasma power results in enhanced degree of sintering for composite spray dried powders. Hence, higher degree of consolidation, both between (i) CNTs and $\mathrm{Al}_{2} \mathrm{O}_{3}$ interface and ii) $\mathrm{Al}_{2} \mathrm{O}_{3}-\mathrm{Al}_{2} \mathrm{O}_{3}$ interface leads to reduced porosity content in A4C-SD and A8C-SD coatings (when compared to that of A4C-B coating. Thereby, synergetic densification of coatings (in addition to CNT addition and dispersion) assisted in enhanced fracture toughening of plasma sprayed nanocomposite coatings.

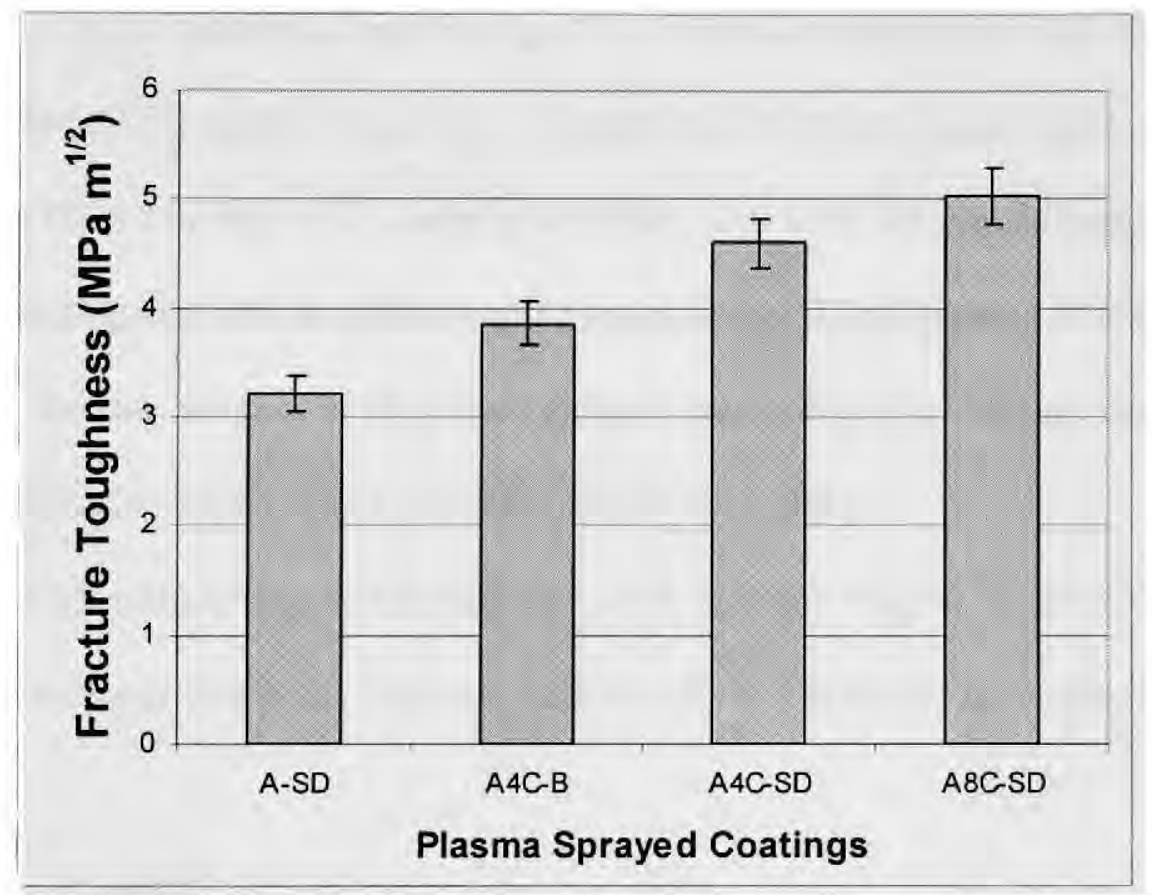

Fig. 4.30: Fracture toughness of plasma sprayed coatings.

To solely estimate the role of porosity in evaluating the fracture toughness of ceramic nanocomposite coatings, precise control on porosity should be required without disturbing (i) porosity size, and (ii) porosity distribution. In order to estimate effect of porosity, processing should prevent CNT damage, retain the grain size, and should not disturb CNT distribution even after consolidation. This in itself is a big challenge. Post 
spray treatment such as hot-isostatic processing (HIPping) is recommended for future endeavors to separate the effect of porosity and CNT content on fracture toughness. Microstructural observation of CNT reinforcement in coatings is explained in the following section.

\subsection{1: Enhancement of Fracture Toughness by CNT Distribution in A4C-B Coating}

Long fibrous-CNT rope structures form anchors between the splats of the aluminum oxide matrix as seen in Fig. 4.31. CNTs are also fused with the molten and resolidified $\mathrm{Al}_{2} \mathrm{O}_{3}$ matrix (Fig 4.31). In addition, CNT form hooks and loops with other CNTs as shown in Fig. 4.32. Looping of CNTs, Fig. 4.31, initiate entangling effect upon tensile loading and aids in restraining the separation of fused regions. This behavior abets superior fracture toughness of plasma sprayed nanocomposite coating. Entangling CNT network has also been observed by other researchers [166].

Tensile stretching of the anchored hook structure, Fig. 4.33, is restrained by high bending stiffness of CNTs. Bending stiffness $(B)$ of CNTs has been largely accepted as [216]:

$$
B=\frac{N E t^{3}}{12\left(1-v^{2}\right)}
$$

where $t$ is the thickness of CNT wall and $N$ is the number of walls in CNT. This clearly implies the direct proportionality of increase in bending stiffness with increasing number of CNT walls in multiwalled carbon nanotubes. Hence, it is expected that loop and hook observed in Fig 4.32 will improve the fracture resistance of $\mathrm{A} 4 \mathrm{C}$-B coating. 


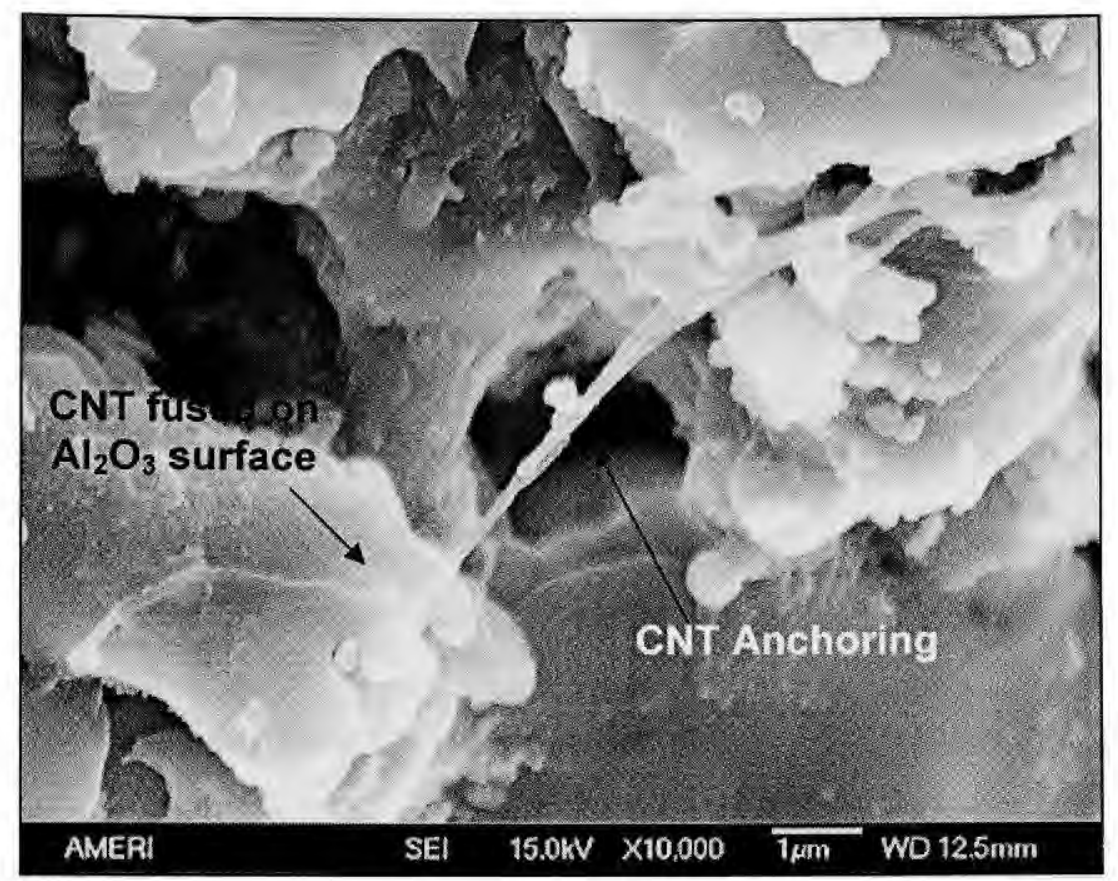

Fig. 4.31: Fibrous CNT fused onto $\mathrm{Al}_{2} \mathrm{O}_{3}$ surface anchoring the splats.

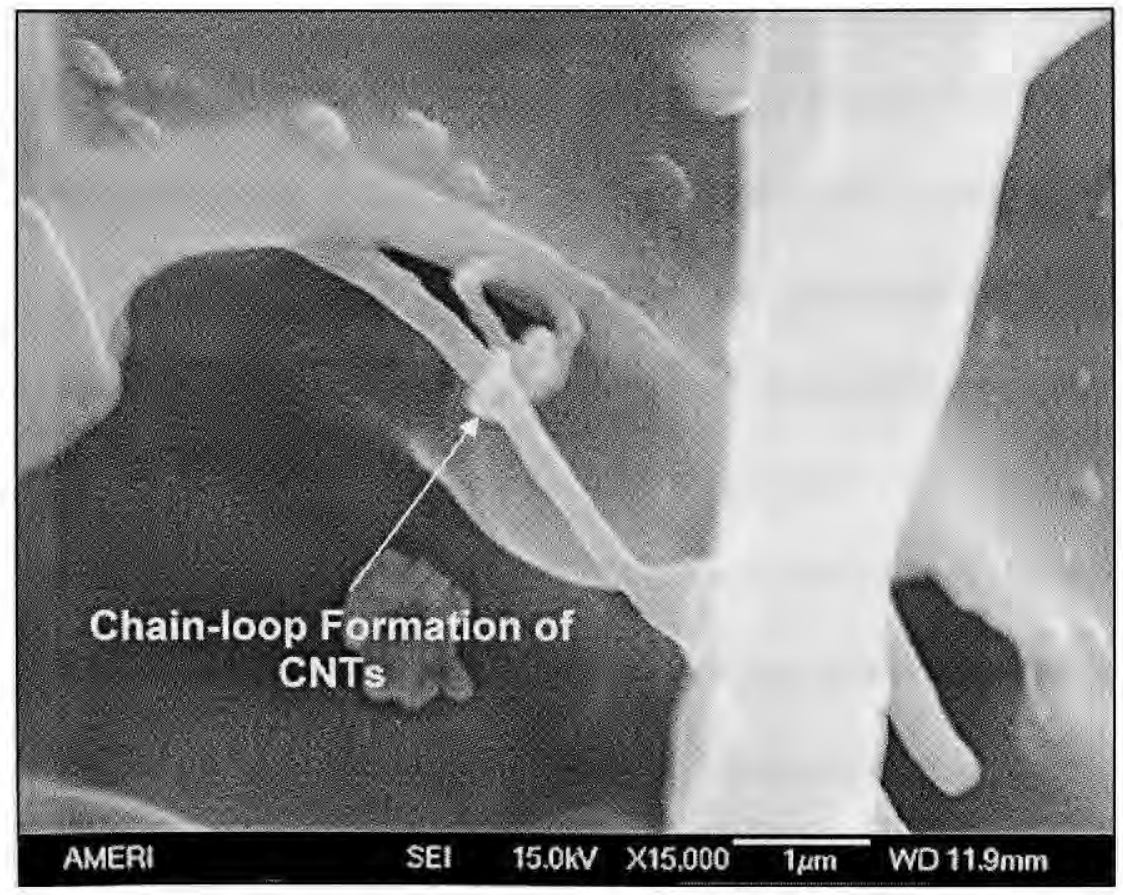

Fig. 4.32: Hook and loop formation of CNTs. 


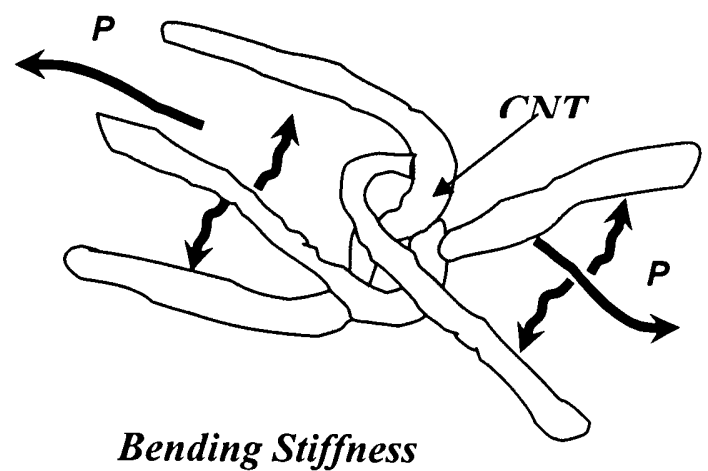

\section{Hook Formation}

Fig. 4.33: Hook formation by entanglement of CNTs in A4C-B coating.

CNT alignment along the splat interface is also observed in A4C-B coating (Fig. 4.34) Since the A4C-B powder has CNTs dispersed only on the surface of the powder, surface melting during plasma spraying gathers most of the CNTs upon the impact onto the substrate. Surface melting of the powder agglomerate eases the CNT movement in the semi-molten/molten pool of $\mathrm{Al}_{2} \mathrm{O}_{3}$. And upon impact onto substrate, CNTs align parallel to the substrate in order to absorb the high collision shock. Consequent impact and deposition of splats entraps CNTs along the intersplat region. Preferential alignment of CNTs brings about the concept of directional strengthening towards enhancing the fracture toughness. Energy absorption upon shock via interfacial sliding also assists in improving the fracture toughness of the coating. Hence, trimodal microstructure (CNTs, partially-melted (PM), and fully-melted (FM) regions) observed in the cross-sectional images of the plasma sprayed nanocomposite coating, Fig. 4.34, rivet fracture toughness enhancement owing to differential phase properties [94].

Interfacial strengthening of the nanocomposite ceramic can be realized in terms of load transfer between the CNTs and matrix as shown in schematic Fig. 4.35. Based on the 


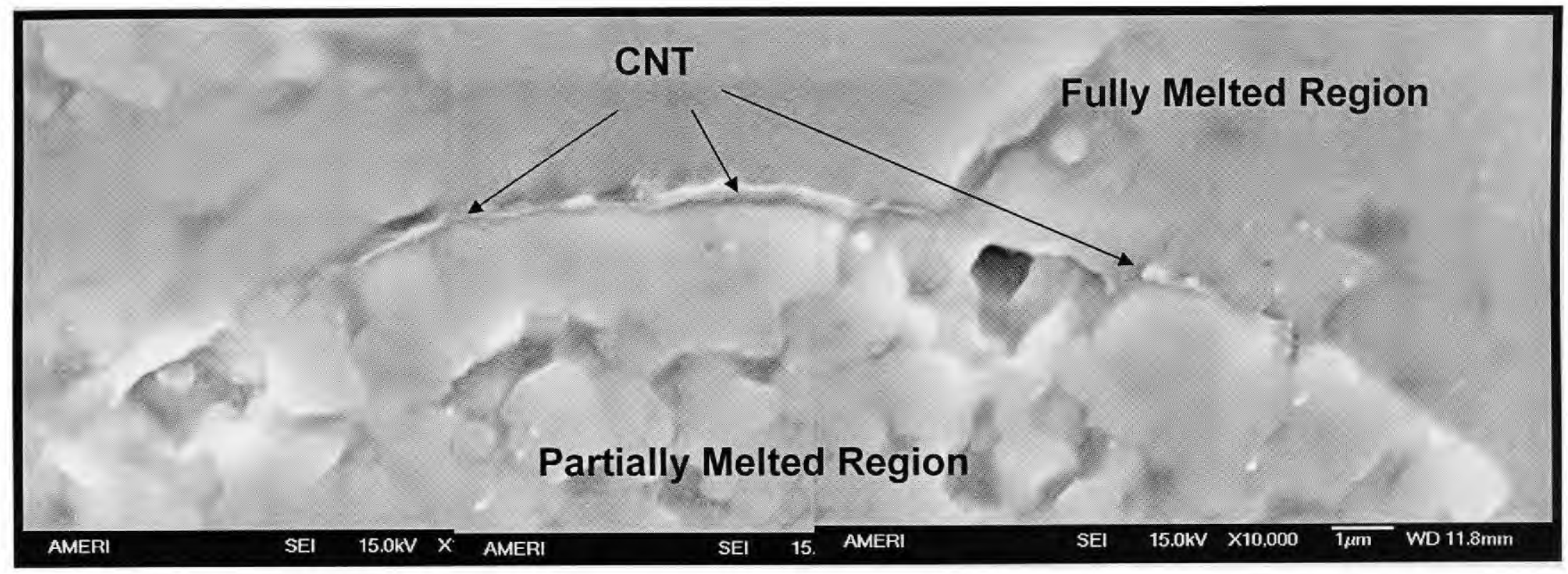

Fig. 4.34: Impact alignment of CNTs along the intersplat region in the plasma sprayed A4C-B Coating. 
assumptions of elastic interfacial shear stress between reinforcement and ceramic matrix, without any sliding [217], modified axial stress (a) and critical shear stress () equations for the parallel aligned MWNT at the interface can be expressed as.

$$
\begin{aligned}
& \sigma=\frac{L^{2}}{L^{2}-r^{2}}\left[\sigma_{C N T}-\frac{r^{2}}{L^{2}}\left(\frac{E_{C N T}}{E_{\text {splat }}} \sigma_{\text {splat }}\right)\right] \\
& \tau=\frac{r E_{C N T}\left(w_{\text {interface }}-w_{C N T}\right)}{L^{2}\left(1+v_{C N T}\right)}
\end{aligned}
$$

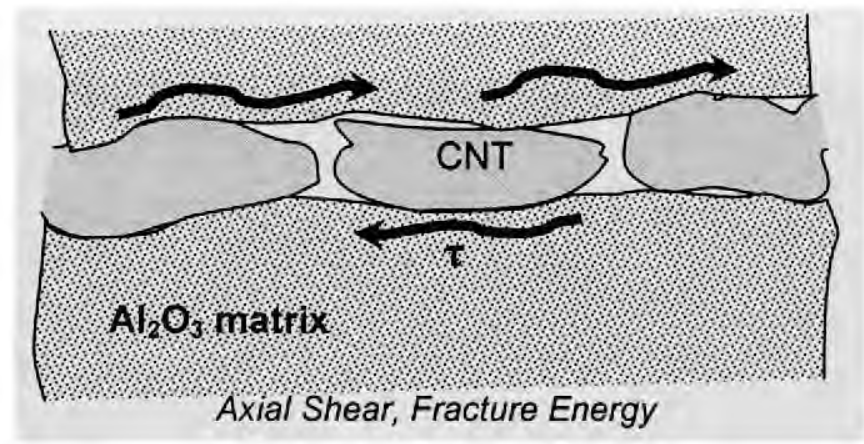

Impact Alignment of CNTs

Fig. 4.35: Increased axial shear and absorption of energy by impact alignment of CNTs along splat interface.

where $r$ is the radial distance, and $L$ is the length of CNT, $w$ is the displacement along axial direction, and $v$ is the Poisson's ratio, and $E$ is the Young's modulus identified with subscripts. Equations 4.6 and 4.7 suggest that parallel alignment of CNTs increase interfacial area and thereby results improved load transfer at the interface and reduction in axial stress $(\sigma)$. Splat interface therefore experiences smooth transitioning of stresses without causing stress concentration. In addition, increased surface area of CNTs 
requires higher critical shear stress $(\tau)$ to debond and pull out from $\mathrm{Al}_{2} \mathrm{O}_{3}$ matrix resulting higher dissipation of fracture energy [217].

Fig. 4.36 shows restraining of the crack damage and consequent crack termination by CNTs. Hence, on one hand, interfacial alignments of CNTs stick the splats, and on the other hand, transverse alignment of CNTs prevents the crack progress. Eventually CNT alignments both along longitudinal and transverse directions contribute to the improved fracture toughness of the plasma sprayed A4C-B nanocomposite coating.

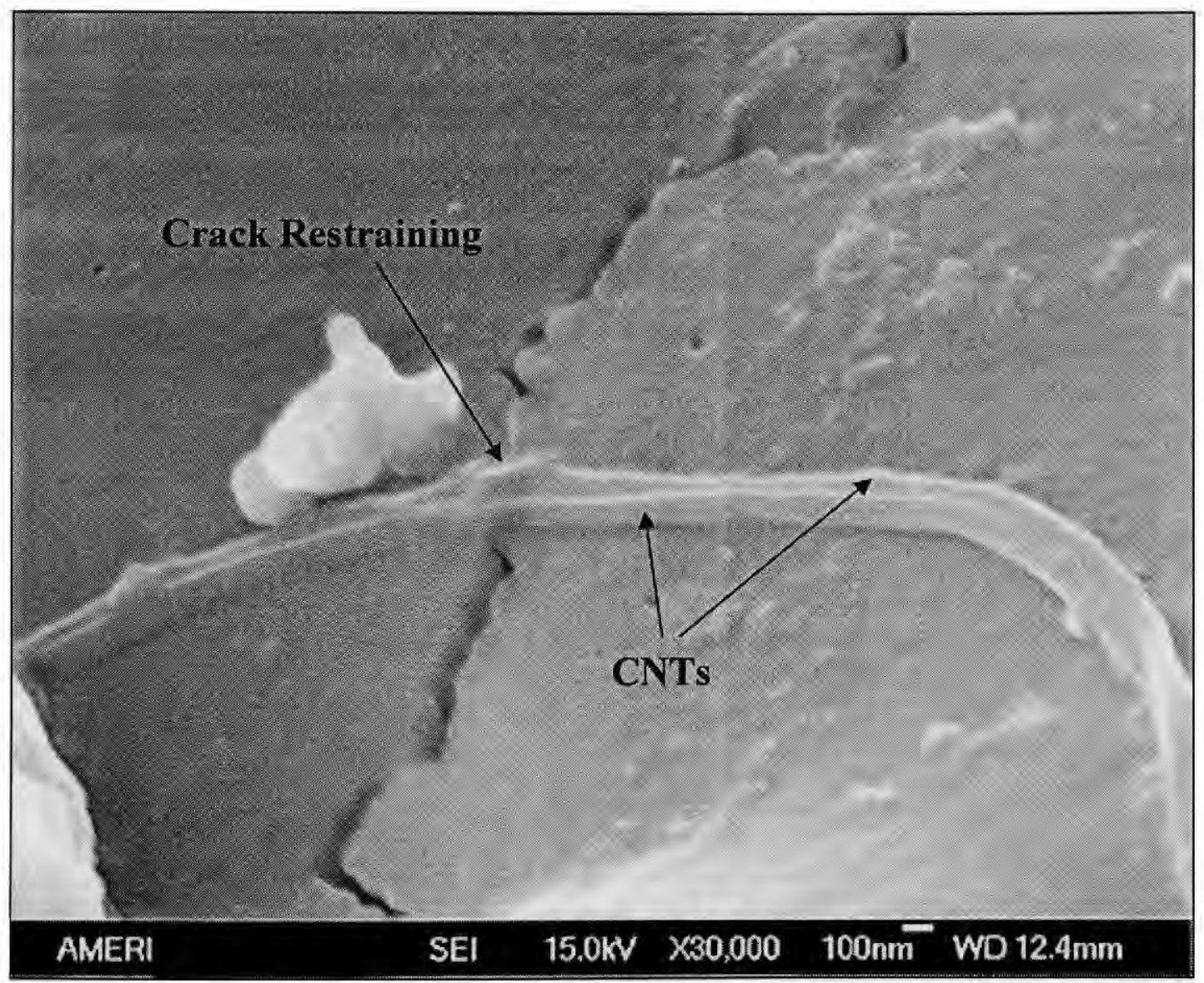

Fig. 4.36: Restraining of crack-damage by presence of CNTs transverse to crack propagation path. 
High magnification SEM image of the A4C-B coating, Fig. 4.37, shows fine (150$250 \mathrm{~nm}) \mathrm{Al}_{2} \mathrm{O}_{3}$ particles attached onto CNT surface. Also, CNTs form a rope structure by aligning with each other. Each $\mathrm{CNT}$ in the rope gets coated with molten $\mathrm{Al}_{2} \mathrm{O}_{3}$. Apart from the melt coating of $\mathrm{Al}_{2} \mathrm{O}_{3}$ on CNT surface, increase in the CNT diameter (Fig. 4.37) to form CNT ropes can be attributed to CNT fusion. Ceramic-coated CNTs easily fuse with the surrounding matrix and show good anchoring with the splats.

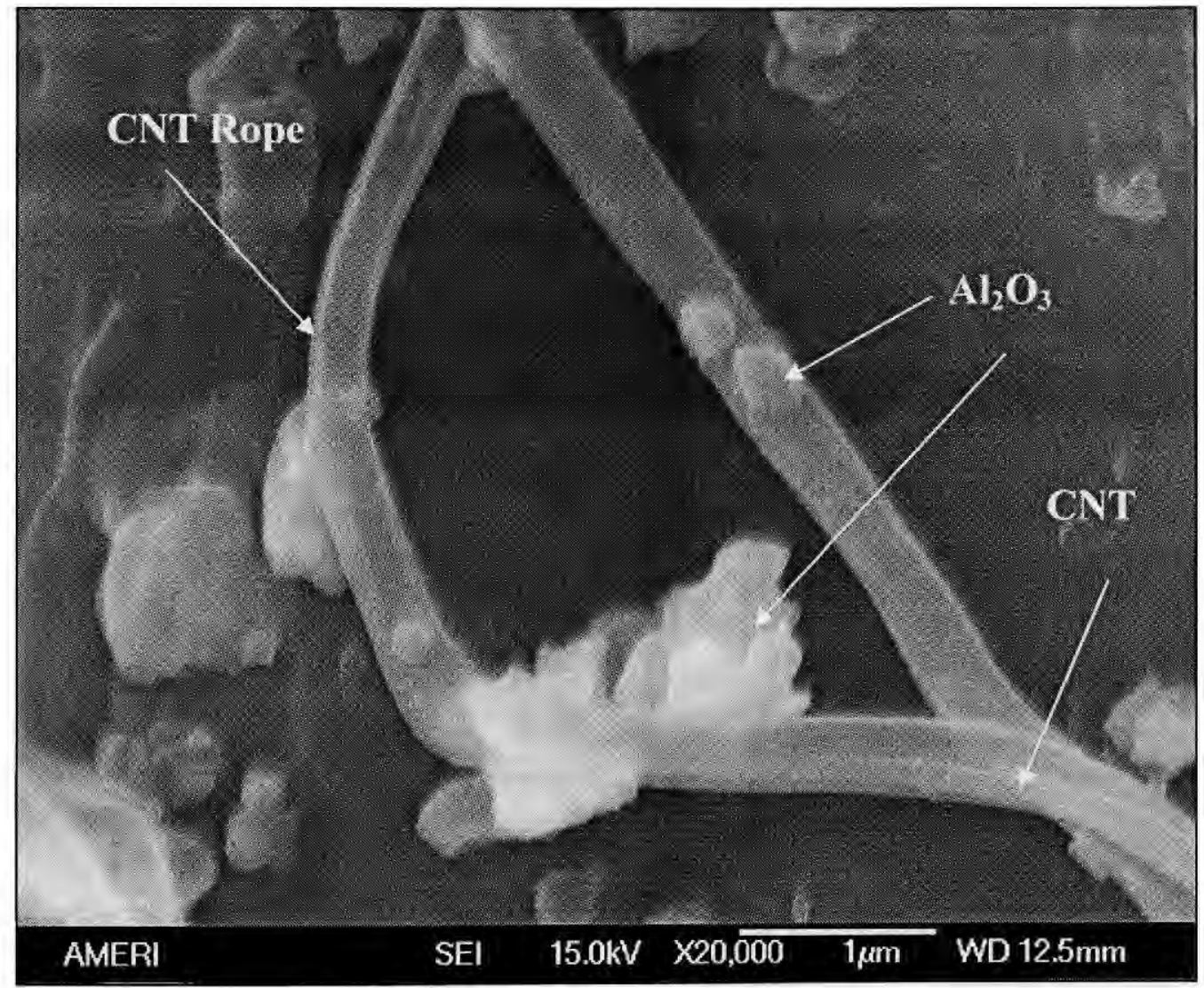

Fig. 4.37: $\mathrm{SEM}$ image of nano $\mathrm{Al}_{2} \mathrm{O}_{3}$ particles sticking to $\mathrm{CNT}$ rope surface in $\mathrm{AC}-\mathrm{B}$ coating. 
Fig. 4.38 is a bright field TEM image and shows features similar to Fig 4.37. Fig. 4.38a shows entanglement of CNTs and $\mathrm{Al}_{2} \mathrm{O}_{3}$ particles hanging on the CNT network. CNT fusion is also observed in Fig. 4.38b indicating superheating of CNTs to fuse with other CNTs. However, CNT structure is intact and not damaged due to fusion. It only forms a CNT network, which will further assist in improving the fracture toughness. Embedded SAD pattern (in Fig. 4.38b) shows diffraction spots corresponding to $\alpha-\mathrm{Al}_{2} \mathrm{O}_{3}$ phase, as indexed in Fig. 4.39. This is indicative of signal arising primarily from large size $(\sim 95 \mathrm{~nm}) \alpha-\mathrm{Al}_{2} \mathrm{O}_{3}$ crystallites $(\sim 68.4 \%)$, (also see Table 4.8$)$.
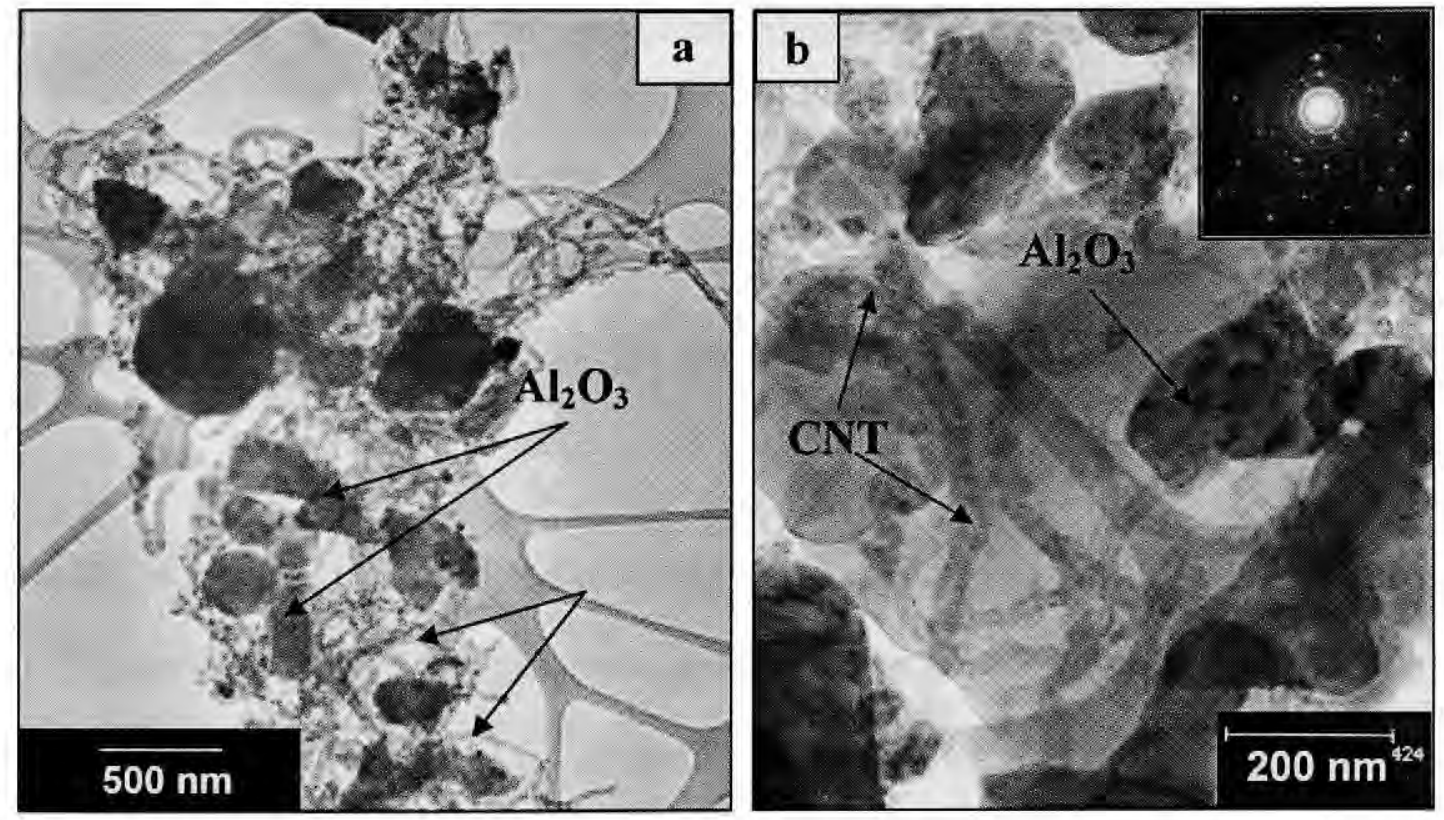

Fig. 4.38: a) TEM micrograph depicting non-preferred dispersion of $\mathrm{Al}_{2} \mathrm{O}_{3}$ particles onto CNT surface, and $b$ ) incomplete sintering of $\mathrm{Al}_{2} \mathrm{O}_{3}$ particles embedded in CNT network and CNT fusion is observed. SAD pattern is embedded along with. 


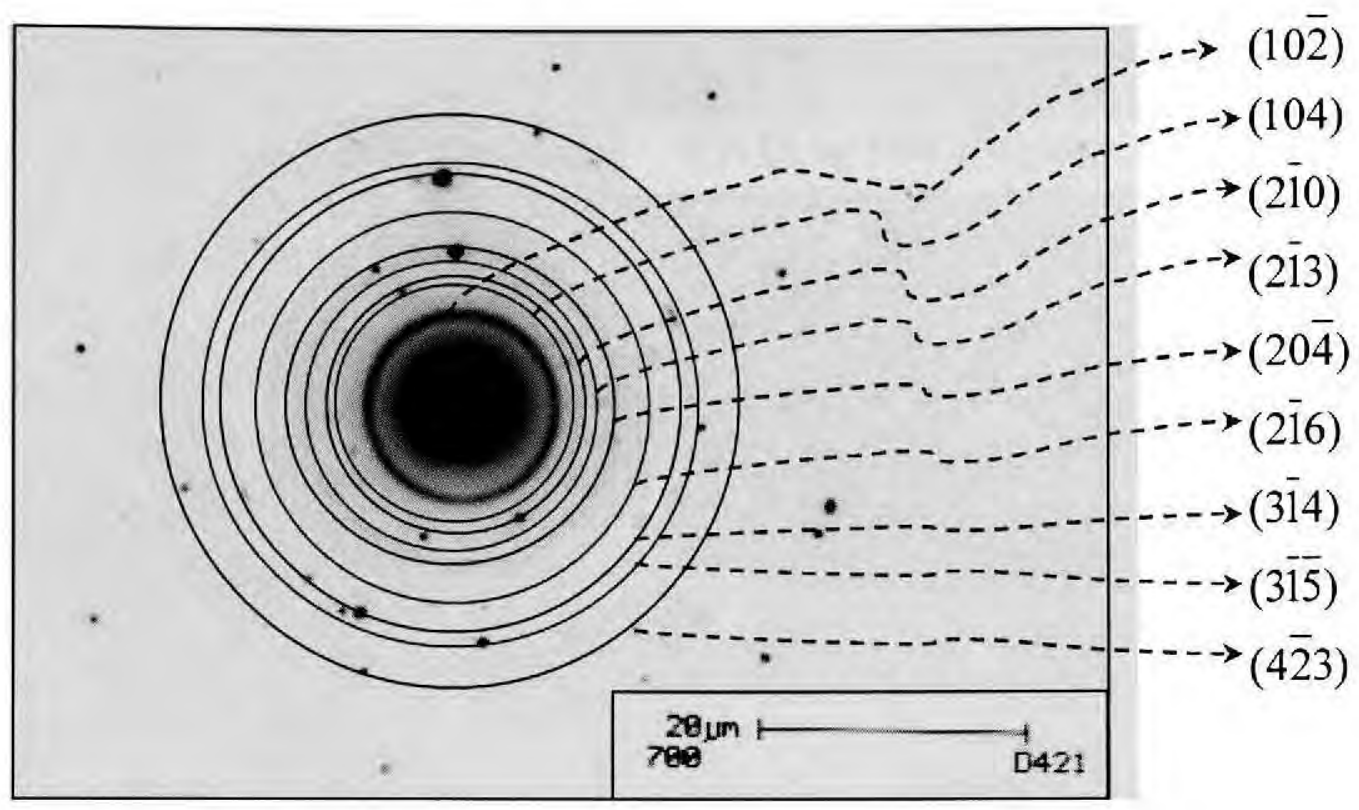

Fig. 4.39: $\mathrm{SAD}$ ring pattern as indexed for major $\alpha-\mathrm{Al}_{2} \mathrm{O}_{3}$ phase.

The role of $C N T$ dispersion in enhancing fracture toughness can be understood by comparing A4C-B coating with A4C-SD coating as content of CNT is same in both coatings. CNT distribution in A4C-SD is explained in the next section.

\subsection{2: Enhancement of Fracture Toughness by CNT Dispersion in A4C-SD Coating}

Figure 4.40 shows CNT dispersion in all the regions of fully melted, partially melted and retained nano $\mathrm{Al}_{2} \mathrm{O}_{3}$ regions of $\mathrm{A} 4 \mathrm{C}-\mathrm{SD}$ coating. Presence of CNTs throughout the matrix shall provide homogeneous and consistent properties of the coatings. A4C-SD coating shows additional toughening mechanisms such as CNT pinning of splats, CNT bridge formation and CNT mesh formation. 


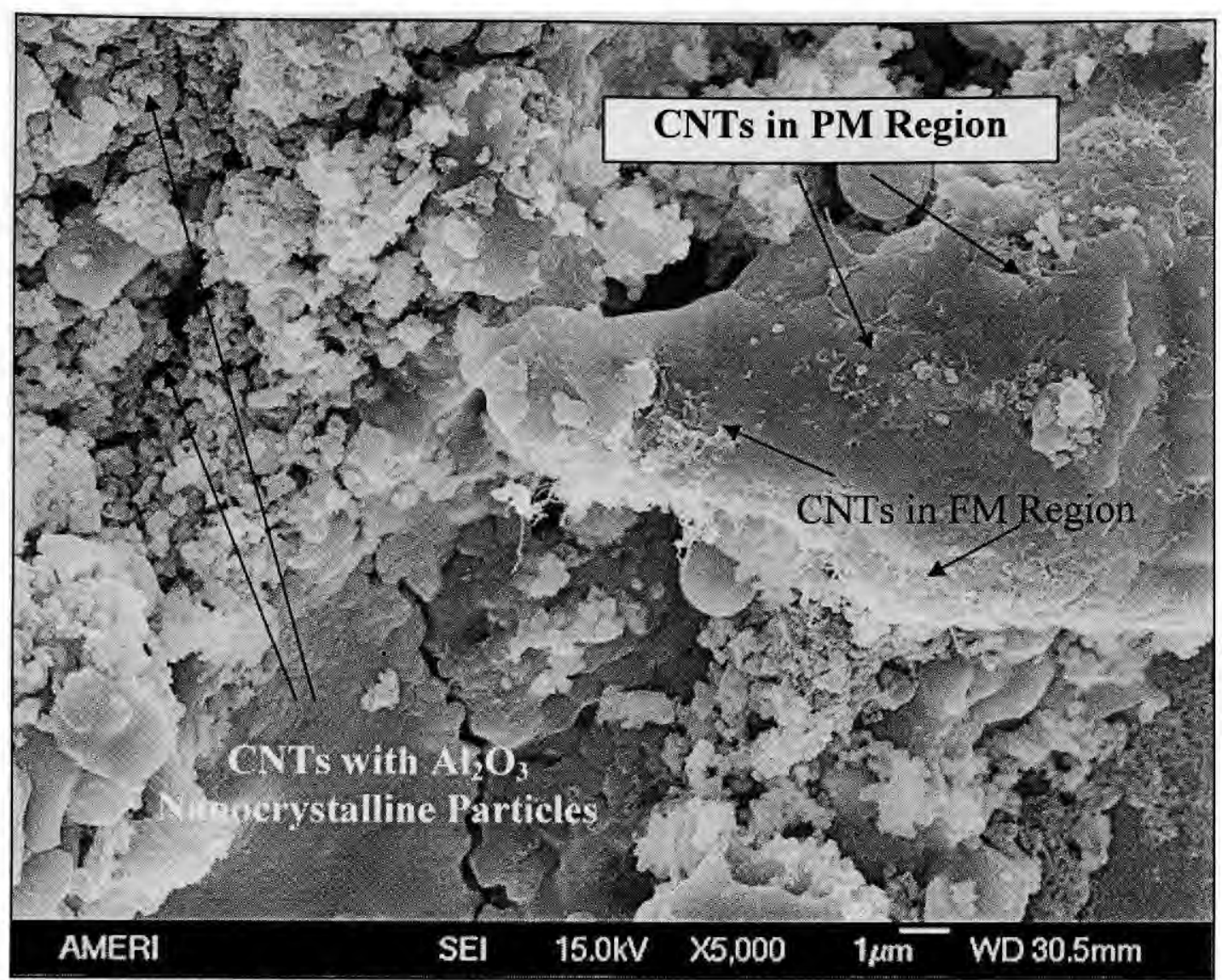

Fig. 4.40: $\mathrm{CNT}$ retention and dispersion is observed in the PM, FM and nano- $\mathrm{Al}_{2} \mathrm{O}_{3}$ regions.

CNTs are present on the surface, in between the splat regions, and embedded in the melted region of plasma sprayed A4C-SD coating, Fig. 4.41. CNTs entrapped in the fully melted region indicate their survival in the plasma plume and consequent retention in the deposited coating. CNTs in the fully molten region can directly add onto the excellent mechanical properties. CNTs present on the surface assist shock absorption during impact and serve as energy absorption regions to result enhanced fracture toughness. CNTs embedded between the splat regions serve as glue to hold the splats together and at the same time act as strengthening agents. 


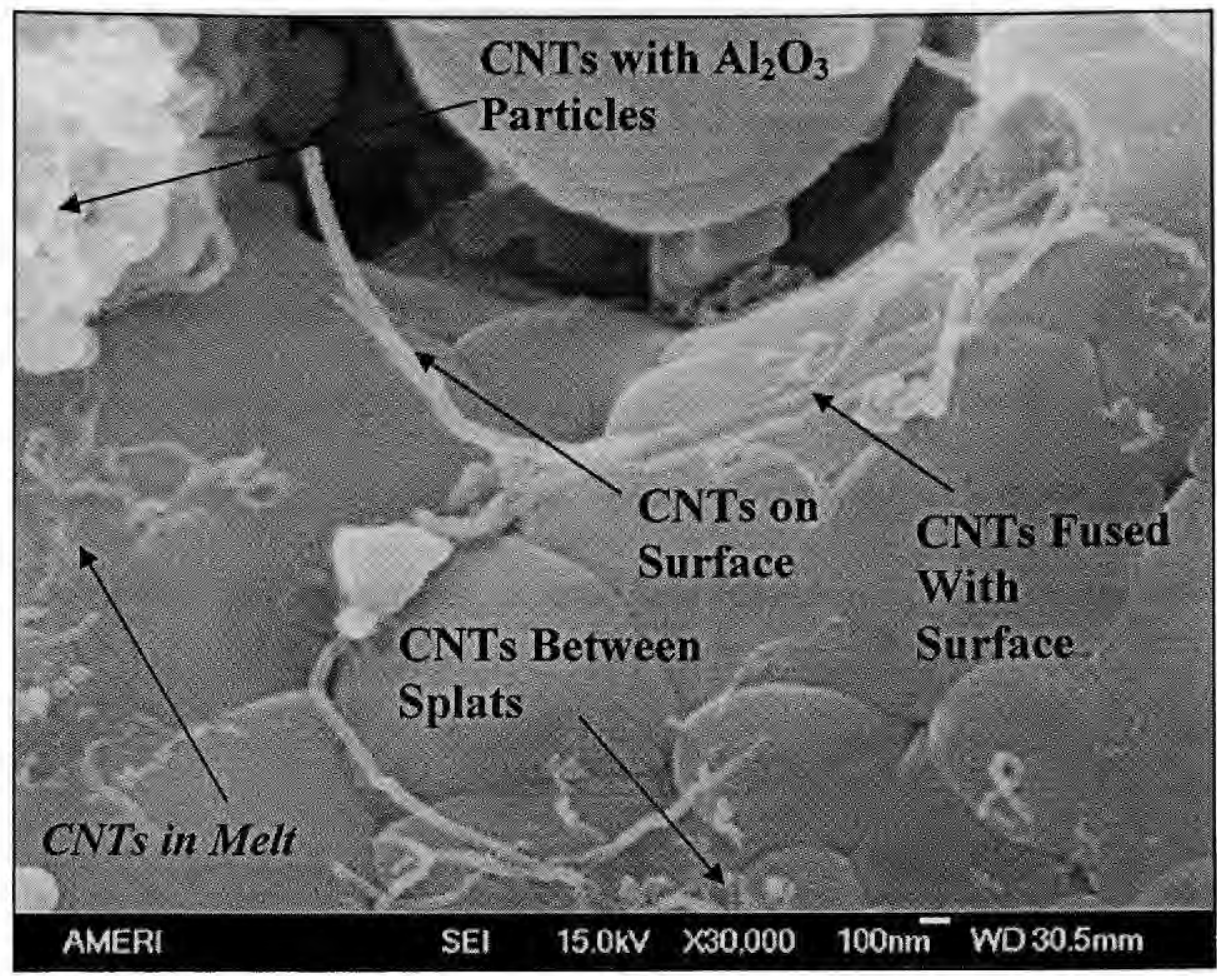

Fig. 4.41: Dispersion of CNTs in FM, PM and splat regions is observed in A4C-SD coating.

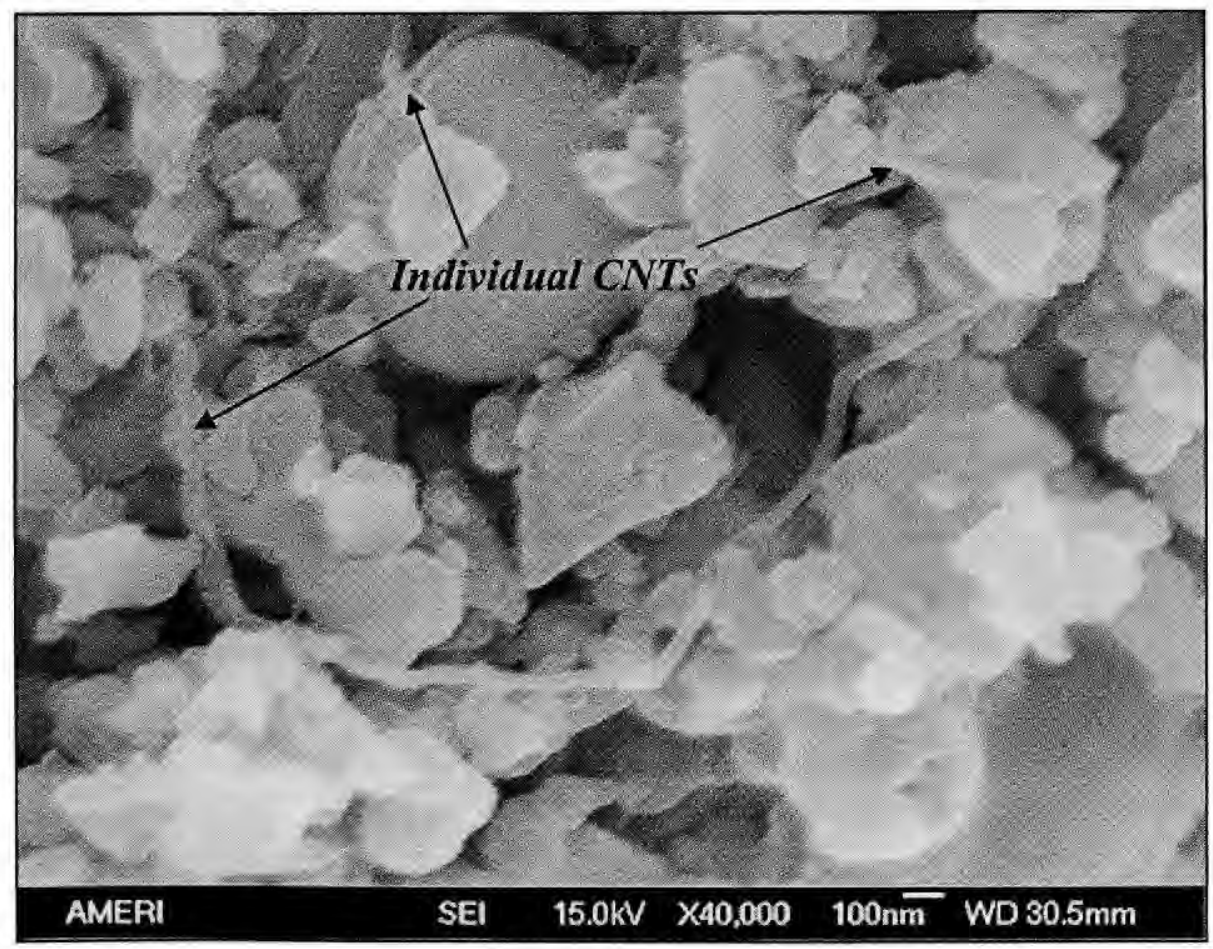

Fig. 4.42: Non-agglomeration of CNTs in the nano- $\mathrm{Al}_{2} \mathrm{O}_{3}$ particle matrix. 
Fig 4.42 shows non-agglomerated $\mathrm{CNT}$ s with the nano $\mathrm{Al}_{2} \mathrm{O}_{3}$ particles. Retention of nanoparticles in the semi-porous region acts as energy absorption sink in terminating the crack-propagation.

Fig. 4.43 shows sintered $n-\mathrm{Al}_{2} \mathrm{O}_{3}$ particles attached to extended CNT emanating from the matrix. $\mathrm{Al}_{2} \mathrm{O}_{3}$ particles are entrapped by curved CNTs indicating strong interaction of the surfaces resulting in enhanced wettability. Wetting of surfaces, therefore, becomes a dominant factor in terms of reinforcing the ceramic nanocomposite.

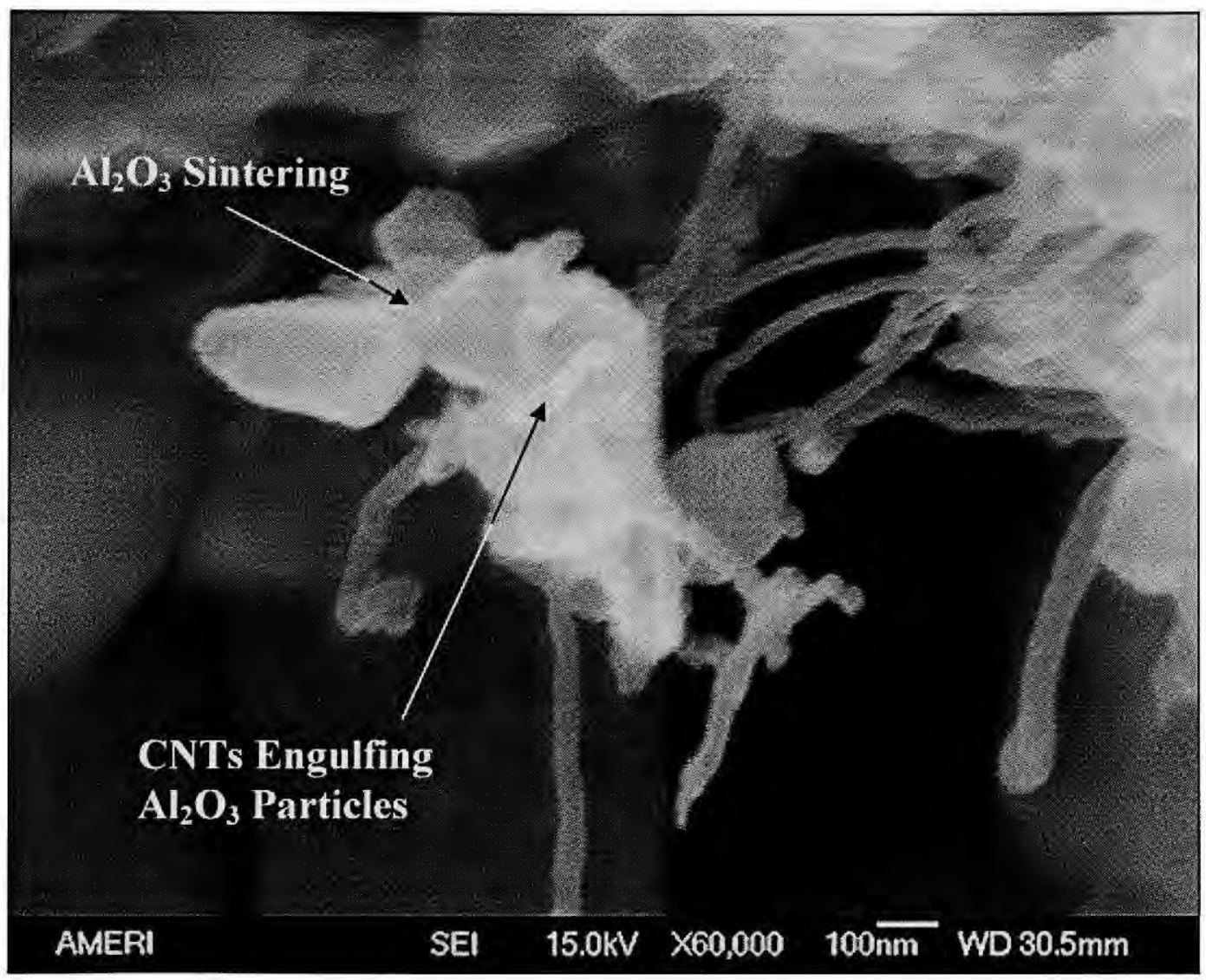

Fig. 4.43: Engulfing of $\mathrm{Al}_{2} \mathrm{O}_{3}$ particles by CNTs. $\mathrm{Al}_{2} \mathrm{O}_{3}$ sintering is also observed in the SEM image. 
Fig. 4.44 shows a CNT bridge between matrix splats. Thickening of CNT bridge is caused by the flow of molten $\mathrm{Al}_{2} \mathrm{O}_{3}$ over $\mathrm{CNT}$ surface. Curvature effect at the ends of $\mathrm{CNT}$ is due to the capillary action responsible for covering the CNT along its surface. Excellent wetting of CNT by molten $\mathrm{Al}_{2} \mathrm{O}_{3}$ is evident from Fig. 4.44. Though increased wetting of $\mathrm{CNT}$ with molten $\mathrm{Al}_{2} \mathrm{O}_{3}$ is observed, currently there is no data on the determination of wetting angle [218] and interaction of $\mathrm{Al}_{2} \mathrm{O}_{3}$ and CNTs. The existing literature on $\mathrm{Al}_{2} \mathrm{O}_{3}$-CNT and ceramic-CNT nanocomposite does not refers to wetting phenomenon as these materials are processed via solid state processes such as sintering, spark plasma sintering, and $\operatorname{HIP}[16,172,176,186]$.

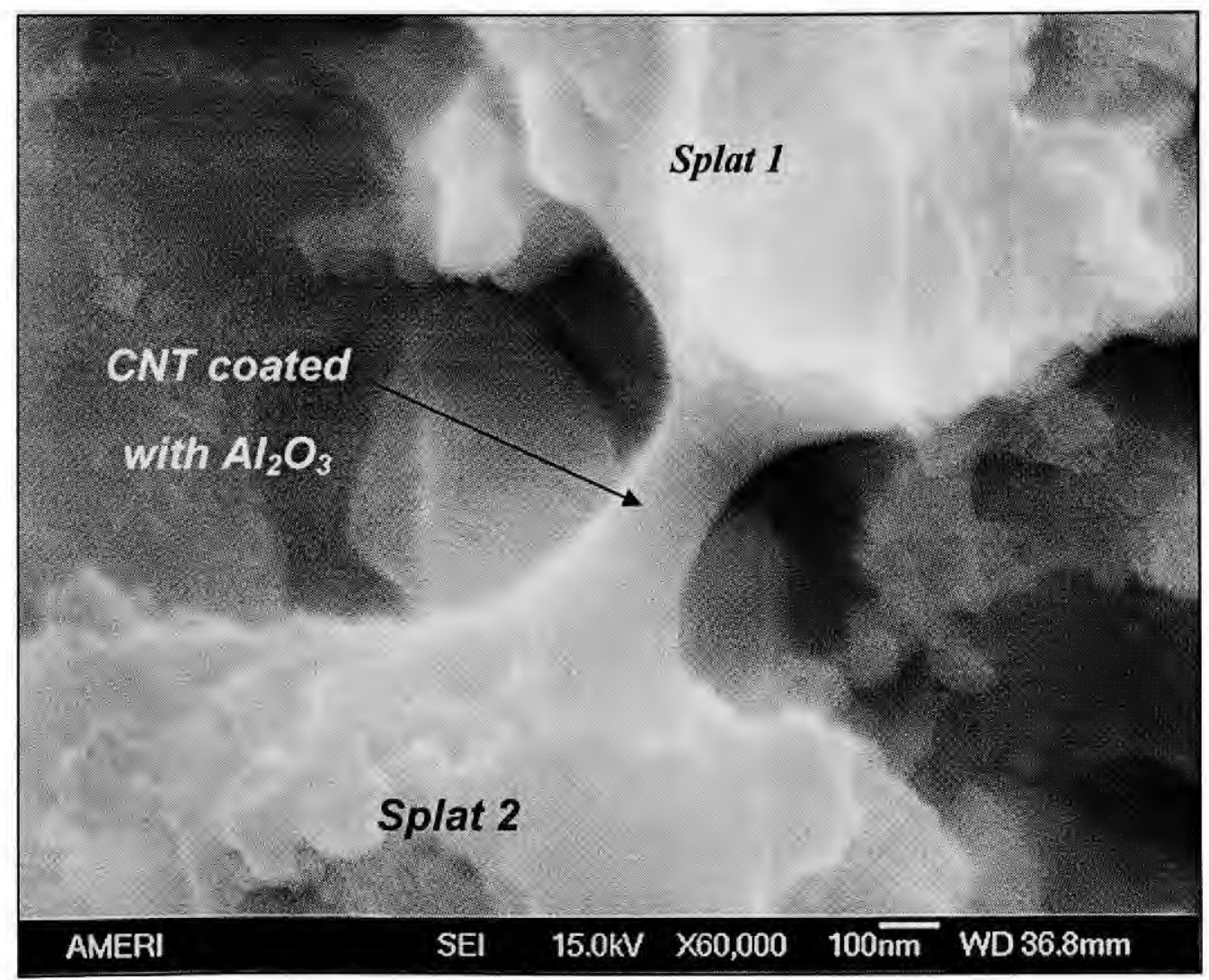

Fig. 4.44: CNT bridge formation between two splats in A4C-SD coating. 
Fig. 4.45 shows an interesting phenomenon of CNT mesh formation in A4C-SD coating which can be explained in terms of wetting kinetics. Surface tension holds the molten $\mathrm{Al}_{2} \mathrm{O}_{3}$ over $\mathrm{CNT}$ surface as a coating during plasma spraying. Since increased contact area leads to reduced surface energy, $\mathrm{Al}_{2} \mathrm{O}_{3}$ coated CNTs come closer to each other. As the volume of molten $\mathrm{Al}_{2} \mathrm{O}_{3}$ over CNTs increases, surface tension is not large enough to hold the molten liquid in place. Now capillarity has enough time to allow seeping down of molten surfaces to leave behind a meshed network of CNTs, Fig. 4.45 $[197,219]$. These phenomena have been validated by modeling the $\mathrm{CNT}-\mathrm{Al}_{2} \mathrm{O}_{3}$ interface, which is explained in the later section.

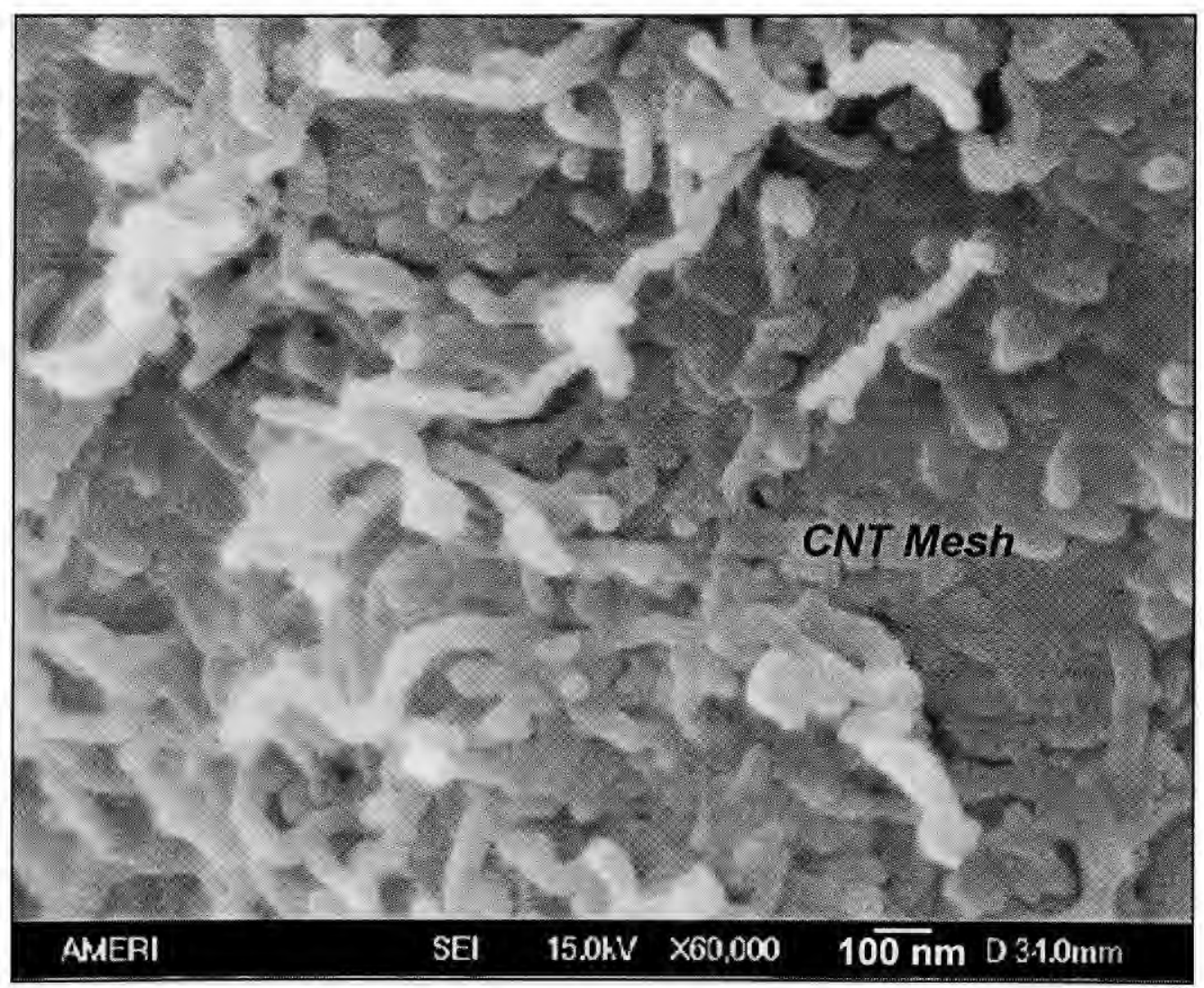

Fig. 4.45: CNT mesh formation observed due to capillarity in the plasma sprayed A4C-SD coating. 
TEM image of plasma sprayed A4C-SD coating, Fig. 4.46a, shows a single dispersed CNT anchored at $\mathrm{Al}_{2} \mathrm{O}_{3}$ surface. This is in contrast with $\mathrm{A} 4 \mathrm{C}-\mathrm{B}$ (Fig. 4.38) coating that shows agglomerated CNTs. Enhanced fracture toughness observed for A4CSD coating is attributed to improved dispersion of CNTs in the matrix, along with enhanced wetting of CNT surface by molten $\mathrm{Al}_{2} \mathrm{O}_{3}$ during plasma spraying. Embedded SAD pattern depicted presence of two phases, viz. $\alpha-\mathrm{Al}_{2} \mathrm{O}_{3}$ and $\gamma-\mathrm{Al}_{2} \mathrm{O}_{3}$. An enlarged image of the SAD pattern is shown in Fig. 4.46b.

Diffraction spots from $\gamma-\mathrm{Al}_{2} \mathrm{O}_{3}$ are observed as secondary diffraction away from the primary transmitted beam, Fig. $4.46 \mathrm{~b}$. One of the diffraction spots of $\alpha-\mathrm{Al}_{2} \mathrm{O}_{3}$ is acting as primary beam for diffraction of $\gamma-\mathrm{Al}_{2} \mathrm{O}_{3}$ crystallites. Presence of dual phases is indicative of precipitation of fine $\gamma-\mathrm{Al}_{2} \mathrm{O}_{3}$ crystallites $(\sim 15 \mathrm{~nm})$ in the $\alpha-\mathrm{Al}_{2} \mathrm{O}_{3}$ matrix $(\sim 73 \mathrm{~nm})$.

Vicker indentation radial crack as observed in Fig. $4.47 \mathrm{a}$ is restrained by CNT bridges across the propagating crack in A4C-SD coating [220]. Bridge structure reinforces the splat separation during crack propagation leading to topsy-turvy crack path. Hence, higher energy absorption regulates the reduced crack length to reinforce the integrity of the structure. Herein role of dispersed CNTs in A4C-SD coating (Fig. 4.40 and Fig. 4.41) provides multiple sites of anchoring and bridging the splats (Fig. 4.44).

Solid-state sintered region, Fig. 4.47b, (also Fig. 4.42) fulfils the objective of terminating the propagating crack by providing the necessary sink for energy relaxation. Hence, combination of powder treatment to uniformly distribute CNTs, and controlling the plasma parameters to obtain bimodal microstructure can greatly influence improvement in the fracture toughness of the plasma sprayed nanocomposite coating. 

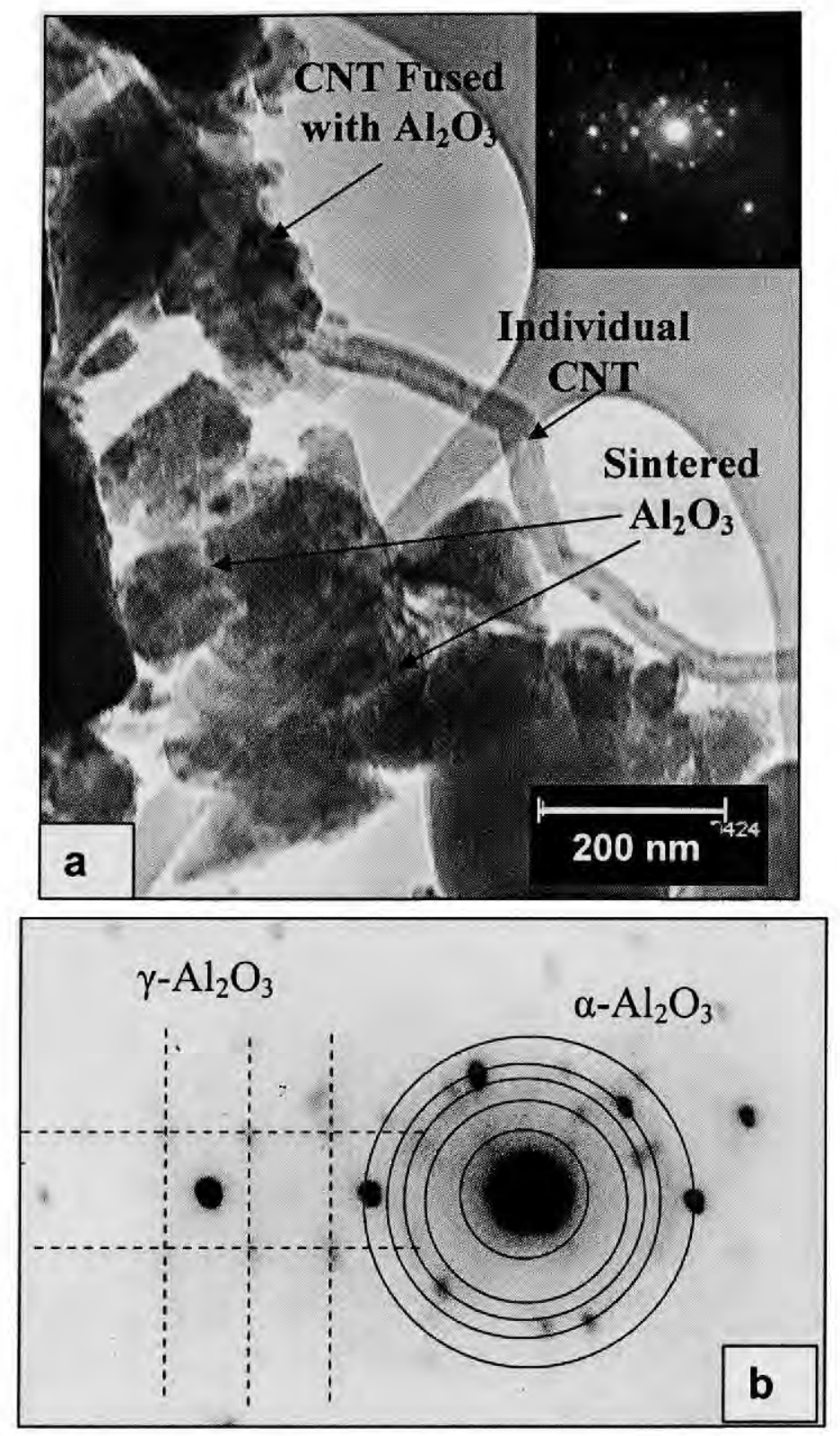

Fig.4.46: a) TEM image of dispersed $\mathrm{CNT}$ in $\mathrm{Al}_{2} \mathrm{O}_{3}$ matrix showing $\mathrm{CNT}$ fusion with $\mathrm{Al}_{2} \mathrm{O}_{3} . \mathrm{Al}_{2} \mathrm{O}_{3}$ particle sintering is also observed. Embedded $\mathrm{SAD}$ pattern is enlarged, and b) dual crystalline phases are seen in the diffraction image. 


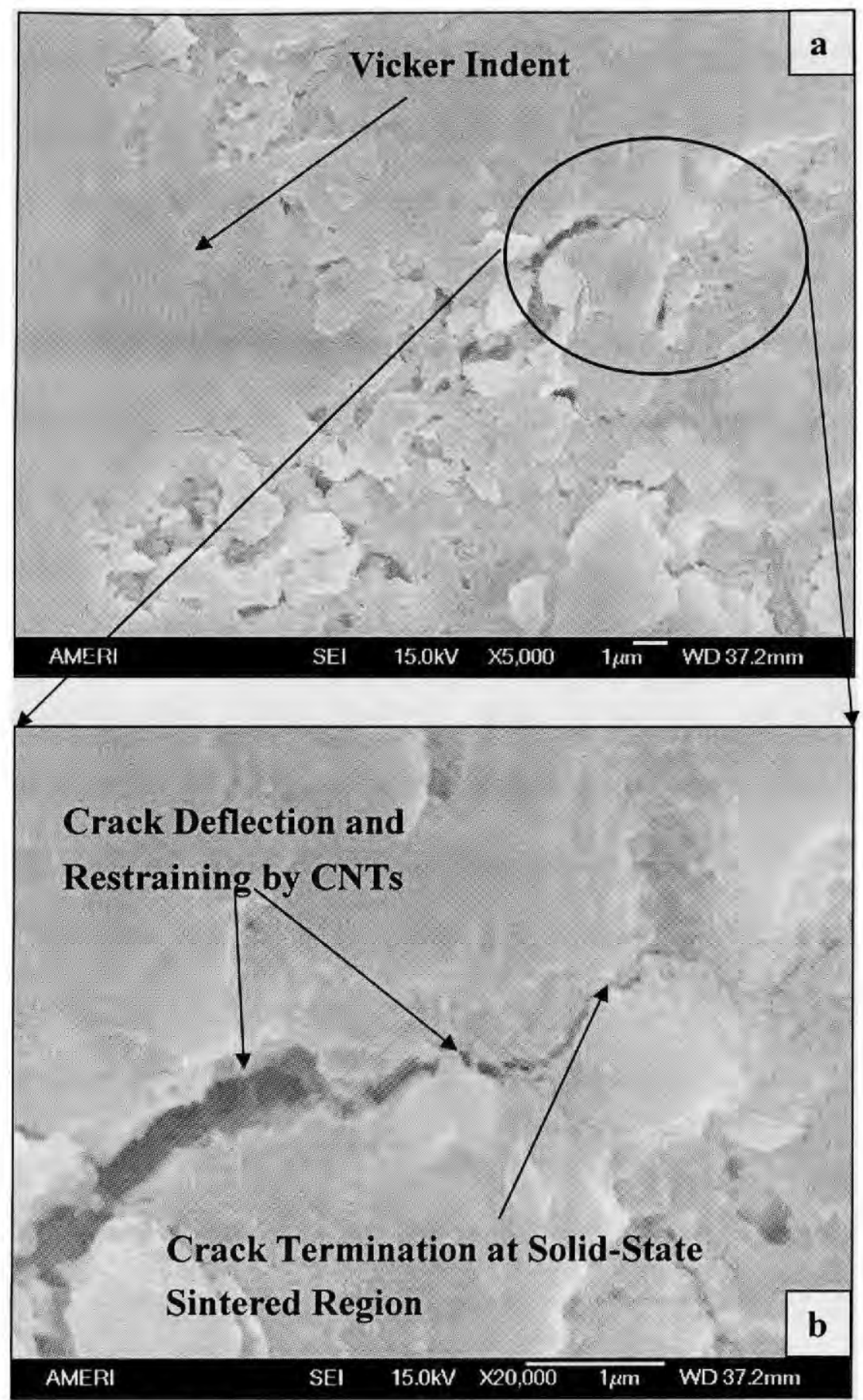

Fig. 4.47: Vicker indentation crack in A4C-SD coating depicting: a) radial crack generation, and b) crack deflection and restraining by CNTs and crack. termination at solid state sintered region. 


\subsection{3: Enhancement of Fracture Toughness by CNT Dispersion in A8C-SD Coating}

Plasma sprayed A8C-SD coating shows enhanced CNT content throughout the matrix, i.e., entrapped in the fully melted region, partially melted region and present with nano $\mathrm{Al}_{2} \mathrm{O}_{3}$ particles (Fig. 4.48). In additions, some agglomerated and undispersed CNT are also observed indicating that CNT-agglomeration effect is not counteracted because of increased CNT content. The role of $\mathrm{CNT}$ as reinforcing agent is beneficial in enhancing the toughening of ceramic nanocomposite only in dispersed state. Fig. 4.49 shows clumps of agglomerated CNTs. Agglomeration tendency in the A8C-SD coating is attributed to excessive CNT content ( $8 \mathrm{wt} \%$ ) indicating that the optimized CNT content in the composite spray dried agglomerate should be between 4 wt.\% CNT and 8 wt. \% CNT. Presence of CNTs in the solid state sintered region and presence with nano $\mathrm{Al}_{2} \mathrm{O}_{3}$ grains, Fig. 4.50, still assists in energy absorption upon impact. But it is possible that increase of fracture toughness by addition of CNTs is getting neutralized by CNT agglomeration. This is indicative of further optimization (of uniform CNT dispersion with maximum CNT content) required for excavating a maxima of fracture toughness in the provided composition range.

CNT fusion is also observed in the plasma sprayed A8C-SD coating, Fig. 4.51, resulting in a CNT rope. Though nanotubes are known for offering tensile- and bendtoughness [221], advantage of nanorope CNTs to serve as strong fortification can be extracted efficiently in the nanocomposite coating. In addition to the intra-nanotube sliding potential of graphitic planes in CNTs, superior toughness of CNT-rope in comparison to carbon fiber of equivalent diameter must be realized owing to higher torsion stiffness and inter-nanotube sliding-capability of CNT-rope structure. 


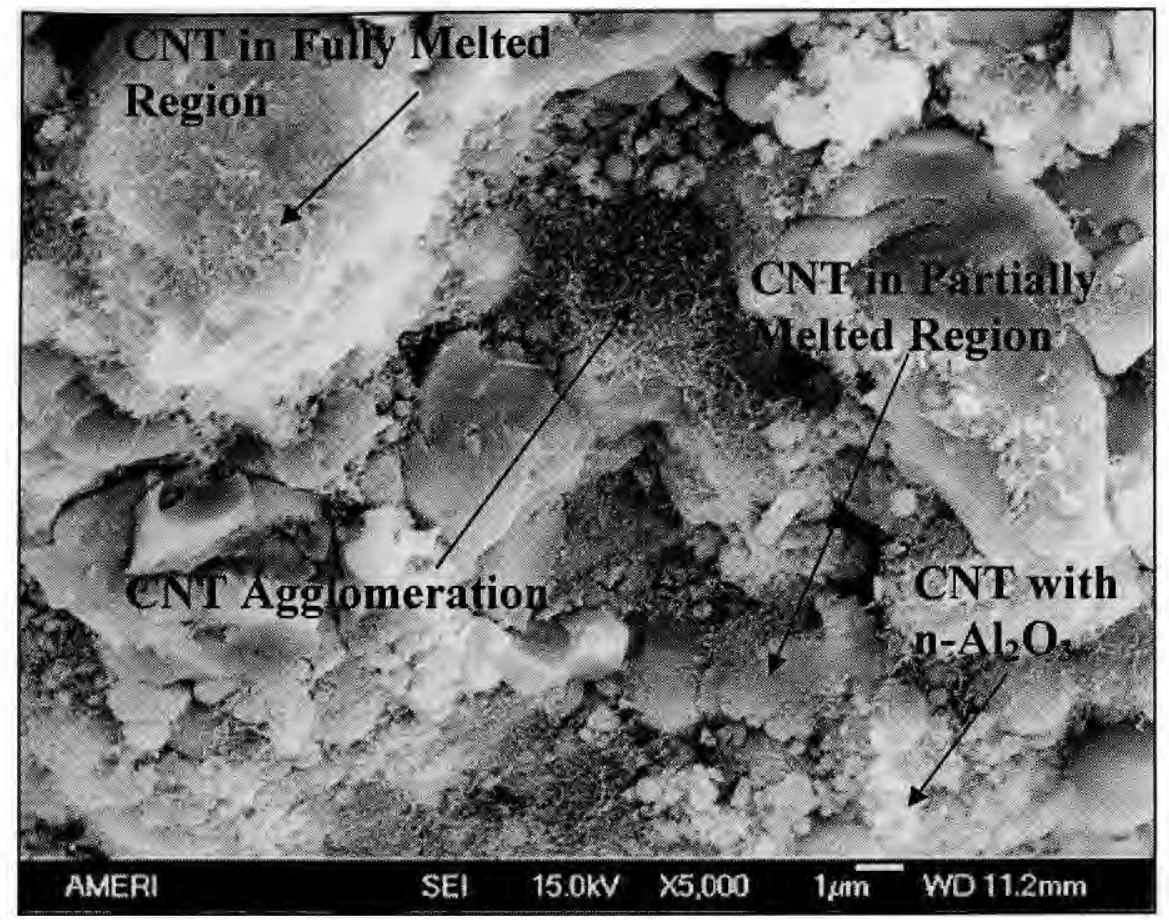

Fig. 4.48: CNT distribution in the plasma sprayed A8C-SD coating.

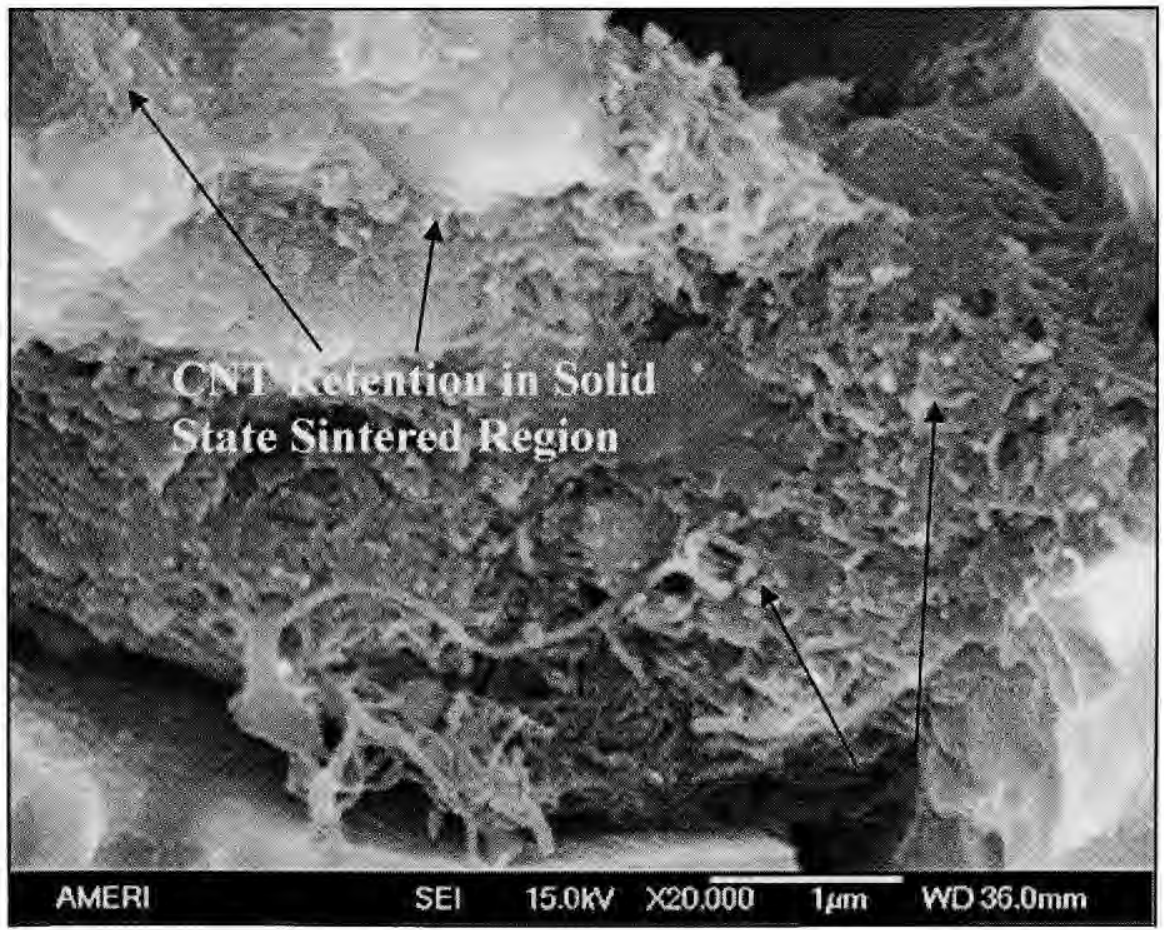

Fig. 4.49: SEM image showing CNT retention and agglomeration in A8C-SD coating. 


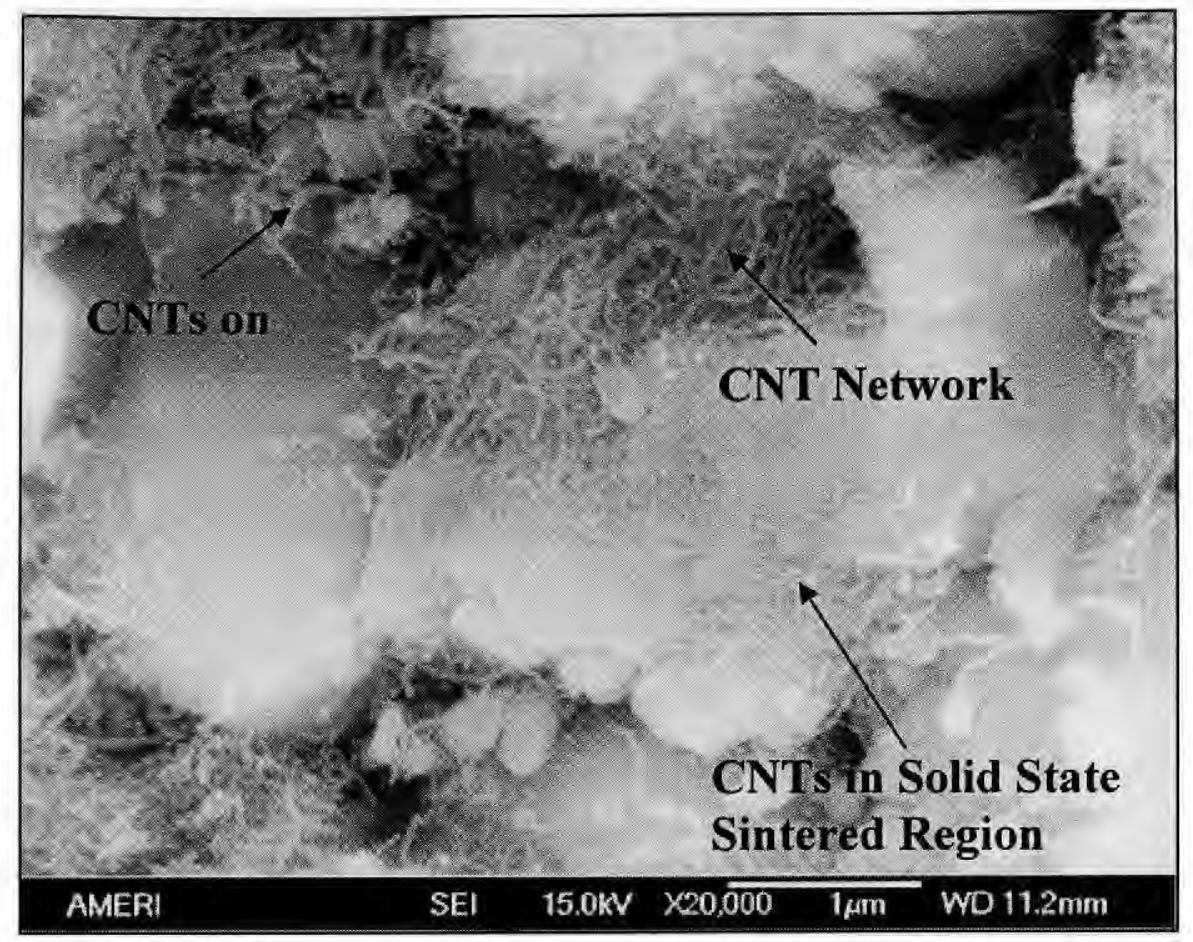

Fig. 4.50: Fracture toughening enhancement via presence of CNTs in solid state sintered region and its presence with nano $\mathrm{Al}_{2} \mathrm{O}_{3}$ particles. $\mathrm{CNT}$ agglomeration as CNT network is also observed.

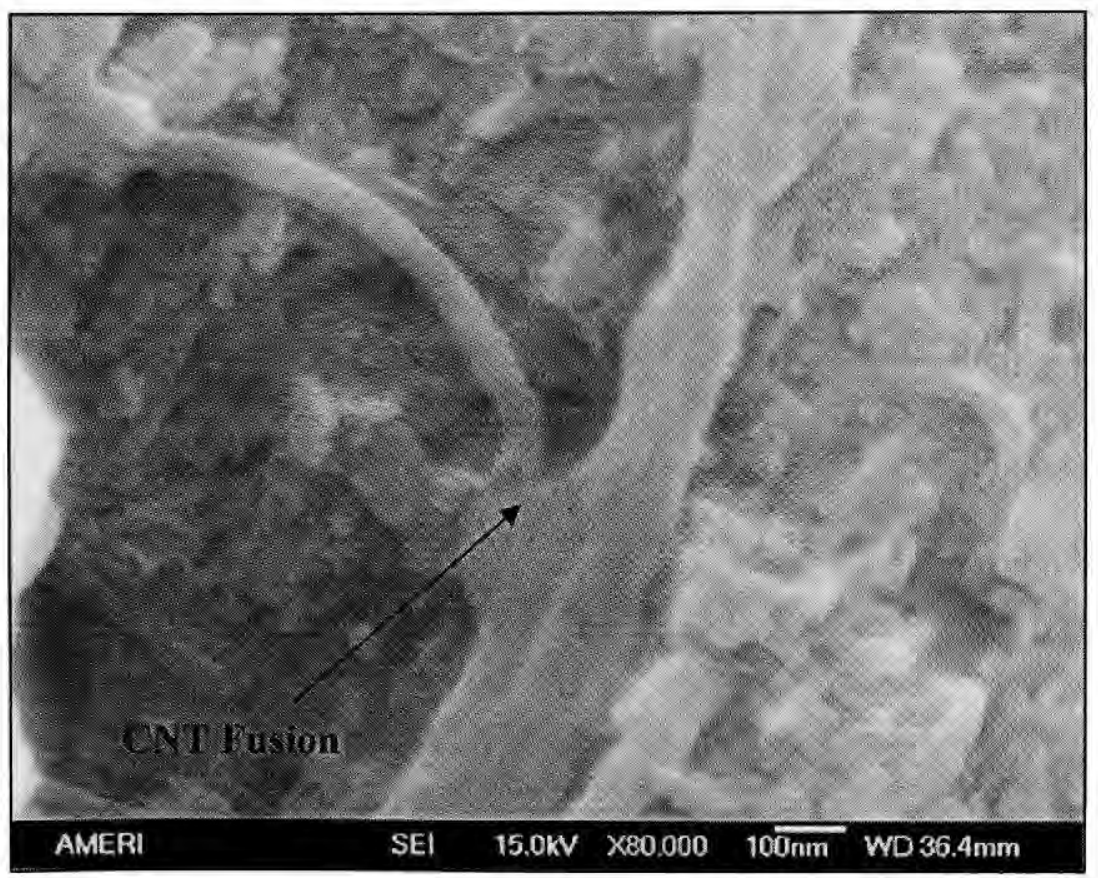

Fig. 4.51: CNT fusion occurring in A8C-SD coating to result a rope structure. 
Schematic of torsional stiffening by rope structure is presented in Fig. 4.52. Shear modulus of nanoropes may be contrasting to that of individual nanotubes since shear in between the nanotubes will dominate over graphitic shear modulus [222]. Intertwining of CNTs induce torsion stiffening upon loading, which improvises the overall toughness of the nanocomposite, Fig. 4.52. Torsion stiffness (K) of CNT is given as [223]:

$$
K=\frac{1}{L} \frac{d^{2} E}{d^{2} \theta}=\frac{r^{3}}{L^{2}} G(2 \pi h)
$$

Equation 4.8

where $\mathrm{Q}$ is torsion angle and $G$ is shear modulus of CNT. Strong dependence of torsion stiffness on CNT-diameter imparts enhanced reinforcing effect to the $\mathrm{Al}_{2} \mathrm{O}_{3}$ matrix making torsion stiffness an essential toughening parameter.

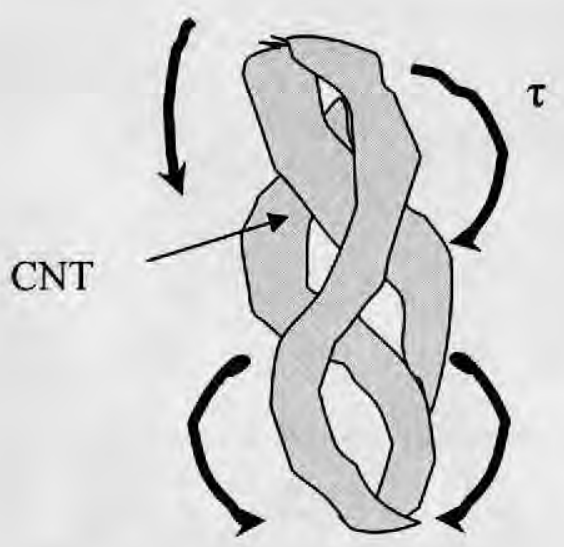

Torsional Stiffness

Fig. 4.52: Schematic of torsional stiffening by CNTs.

TEM of plasma sprayed A8C-SD coating depicts sintering of $\mathrm{Al}_{2} \mathrm{O}_{3}$ particles in Fig. 4.53a. Exaggerated fusion of CNTs is observed in Figs. 4.53a and 4.53b. Entrapment of $\mathrm{Al}_{2} \mathrm{O}_{3}$ particles by CNTs confirms the CNT dispersion by high surface energy of nano- 

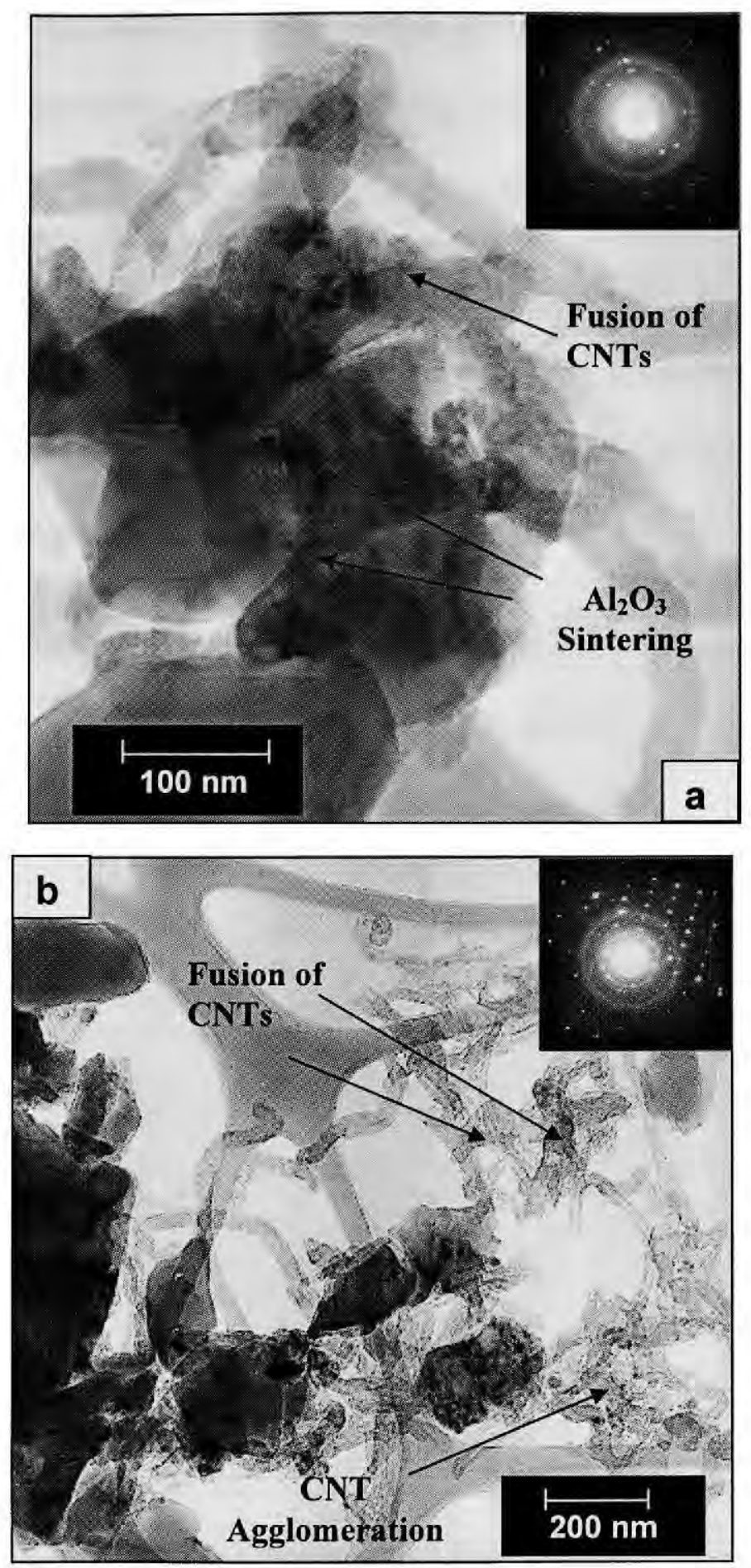

Fig. 4.53: TEM micrograph of A8C-SD coating showing, a) $\mathrm{Al}_{2} \mathrm{O}_{3}$ sintering with embedded $\mathrm{SAD} \alpha-\mathrm{Al}_{2} \mathrm{O}_{3}$ pattern, and b) CNT fusion and agglomeration with embedded SAD pattern of $\gamma-\mathrm{Al}_{2} \mathrm{O}_{3}$. 
$\mathrm{Al}_{2} \mathrm{O}_{3}$ particles. But, excessive agglomeration of CNTs is attributed to the enhanced CNT content in the plasma sprayed A8C-SD coating. Light rings pattern in the embedded SAD image (as two light rings in embedded Figs. $4.53 \mathrm{a}$ and b) correspond to [1010] and [3030] family of planes of CNT. Embedded SAD diffraction pattern in Fig. 4.53a corresponds to $\alpha-\mathrm{Al}_{2} \mathrm{O}_{3}$ (indexed earlier), whereas $\mathrm{SAD}$ diffraction pattern in Fig. $4.53 \mathrm{~b}$ corresponds to $\gamma-\mathrm{Al}_{2} \mathrm{O}_{3}$. Diffraction pattern embedded in Fig. $4.53 \mathrm{~b}$ is indexed in Fig. 4.54, and zone axis of [112] is measured.

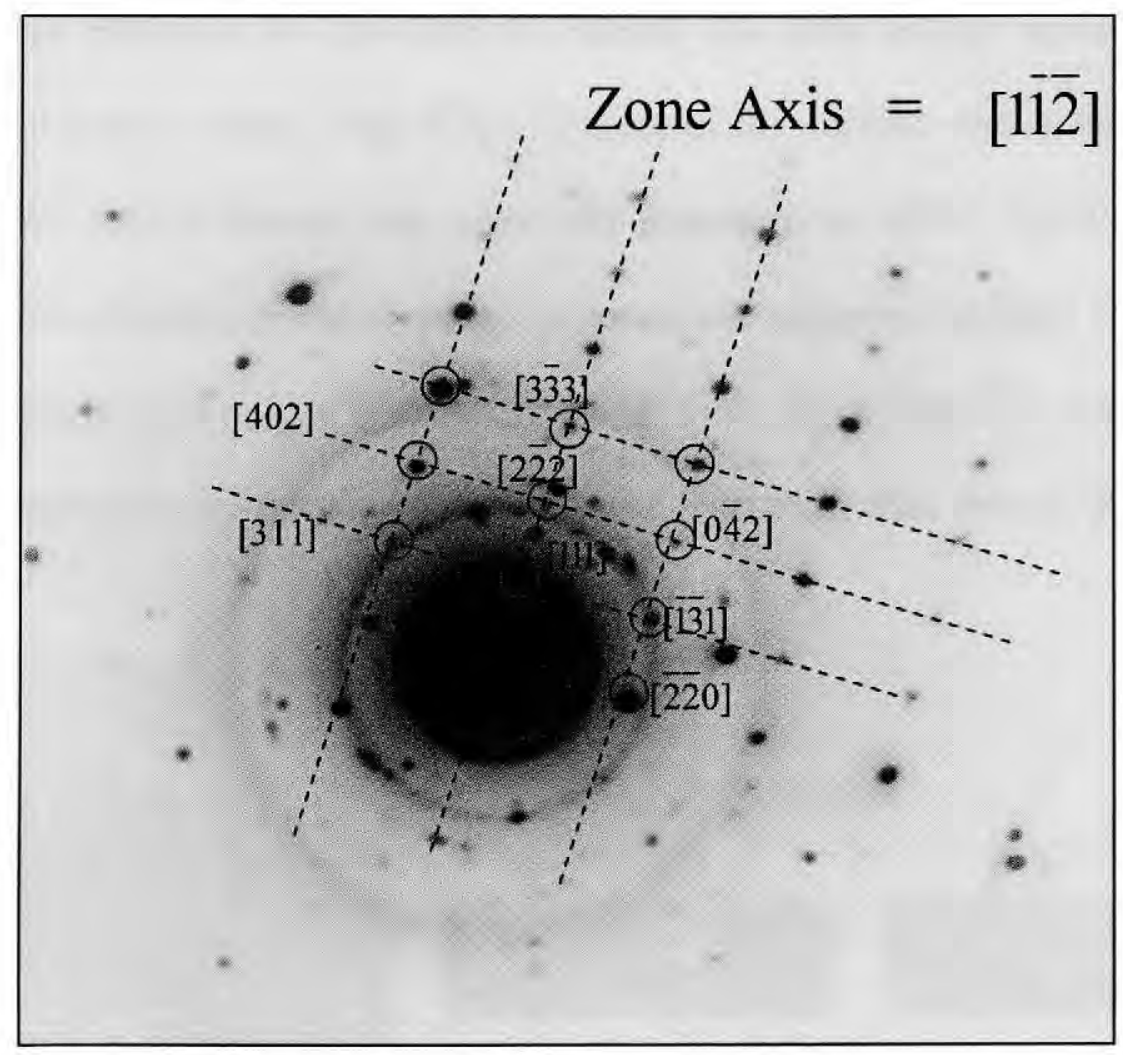

Fig. 4.54: $\mathrm{SAD}$ Diffraction pattern of $\gamma-\mathrm{Al}_{2} \mathrm{O}_{3}$ indexed as zone axis of [112] $]$.

Fracture toughening of the nanocomposite depends on the load transferability from $\mathrm{Al}_{2} \mathrm{O}_{3}$ matrix onto CNTs. $\mathrm{CNT} / \mathrm{Al}_{2} \mathrm{O}_{3}$ interface is critical for stress transfer. 
Toughening mechanisms provided by excellent- bending stiffness (eq. 4.5), axial and shear strength (eqs. 4.6 an d 4.7), and torsional stiffness (eq. 4.8) can play a part in the presence of a strong $\mathrm{CNT} / \mathrm{Al}_{2} \mathrm{O}_{3}$ interface. Interface is affected by wettability between the two surfaces. Hence wettability between $\mathrm{CNT}$ and $\mathrm{Al}_{2} \mathrm{O}_{3}$ is an underlying phenomenon towards understanding of the interface properties.

\subsection{Wettability of $\mathrm{CNT}-\mathrm{Al}_{2} \mathrm{O}_{3}$ Interface}

Surface tension is defined as the surface force acting along a line per unit length. Interatomic distances are stretched to balance the extra energy associated with the unsatisfied surface atoms, Fig. 4.55a. Contact of the surface with another body often reduces the surface tension and makes the system more stable. Since the nature has tendency to minimize potential energy, adhesion and cohesion interplay to adjust contact area/angle and equilibrate at dynamically stable state, Fig. $4.55 \mathrm{~b}$. It is clearly evident that when cohesive force between solid and liquid is stronger than that of liquid and vapor
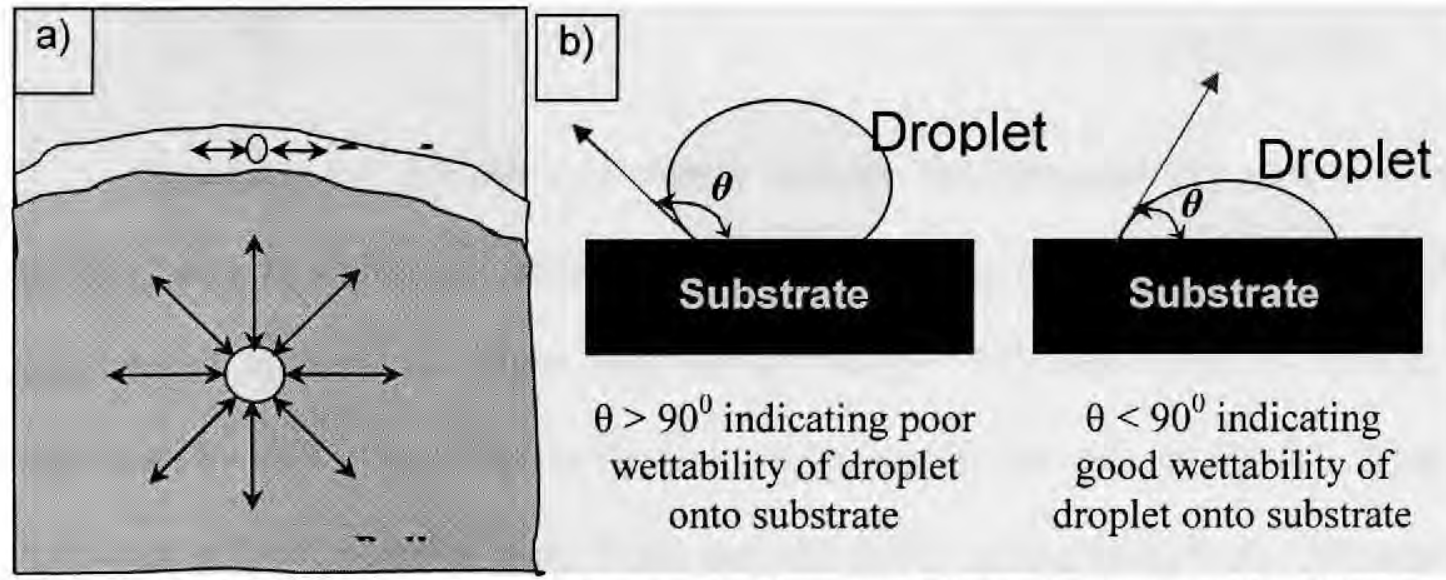

Fig. 4.55: a) High energy at surface results from unbalanced atoms, and b) Wetting characteristic of droplet on a substrate. 
wetting occurs. Wetting is required in realizing good bonding between the substrate and freezing droplet.

It is important to emphasize that high melting points of $\mathrm{Al}_{2} \mathrm{O}_{3}(\sim 2333 \mathrm{~K})$ and CNT $(\sim 3773 \mathrm{~K})$ makes it very difficult to partake liquid droplet angle studies in such a system. Since no theoretical literature is available on such a study, an effort is made to calculate the freezing dynamics involved in the plasma spraying. Surface tension and capillarity are taken as reflective measures of understanding wettability between CNT and $\mathrm{Al}_{2} \mathrm{O}_{3}$. Pressure jump across a curved surface can be defined by Young-Laplace Equation:

$$
\Delta p=\gamma \cdot \frac{d A}{d V}
$$

Equation 4.9

where $\gamma$ is the surface energy at constant pressure and temperature, given as:

$$
\gamma=\left(\frac{d G}{d A}\right)_{P, T}
$$

Equation 4.10

which can be further simplified as

$$
\Delta p=\frac{2 \gamma}{r}
$$

Equation 4.11

Equations 4.9 through 4.11 clearly indicate the enhanced free energy of nano particles due to enhanced surface area (at reduced particle radius). CNTs tend to agglomerate in order to reduce their surface energy. But, this aspect is used to our advantage by dispersing CNTs in the nano- $\mathrm{Al}_{2} \mathrm{O}_{3}$ matrix, fig. 4.4 and fig. 4.5 . Owing to increased surface energy of nano $\mathrm{Al}_{2} \mathrm{O}_{3}$ particles [123], strong seeking and adherence to other surface becomes a requirement to reach stability. Instead of CNT agglomeration, 
now selective agglomeration is negated and surface forces dominate toward uniform distribution of particles (in the nanometer range).

CNTs and nano $\mathrm{Al}_{2} \mathrm{O}_{3}$ particles are seen to distribute without biased agglomeration, Fig. 4.4 and 4.5. The importance of attaching one surface in order to reduce the surface energy is seen via CNT-rope formation in Fig. 4.51. Non-preferential attachment of $\mathrm{Al}_{2} \mathrm{O}_{3}$ and CNTs is observed by scattered particles in TEM micrograph, Fig. 4.38 and 4.53 .

Interfacial studies on alumina-CNT are almost non-existent in the literature owing to increased complexity of ultra high temperatures and solid state processing by other researchers [193]. Since surface is the sole direct contact with surrounding environment, capillarity and surface tension are the only direct measures in defining wettability. A system of $\mathrm{Al}_{2} \mathrm{O}_{3}$ wetting on $\mathrm{CNT}$ is defined through Fig. 4.56. In considering theoretical/computational modeling, it is assumed that molten $\mathrm{Al}_{2} \mathrm{O}_{3}$ layers onto $\mathrm{CNT}$ surface by capillary action, where it freezes instantaneously mimicking the dynamic equilibrium depending on surface tension, 4.57. Rapid kinetics inherent to plasma spraying (dwell time of $\sim 4 \times 10^{-4} \mathrm{~s}$ ) is fast enough to freeze frozen $\mathrm{Al}_{2} \mathrm{O}_{3}$ droplet before it dropped from CNT surface, Fig. 4.57. Effects of gravity are neglected and resulting microstructures are taken as representative model for surface forces towards defining wettability. Since constant equilibrium contact angles are not experimentally observed owing to changing 'true' contact angles, it is a valid assumption to assume dynamic freezing as a representative model [195]. 


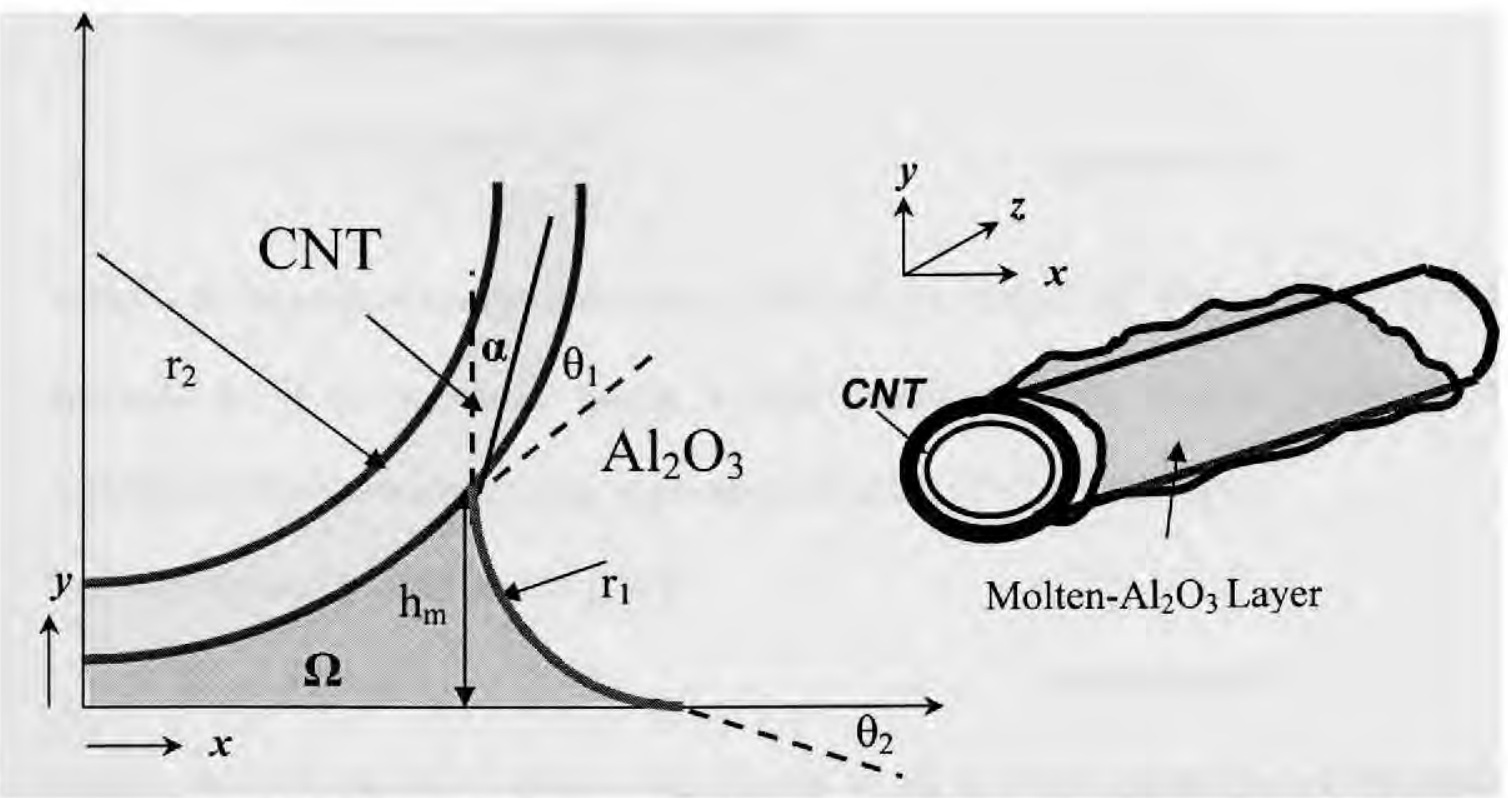

Fig. 4.56: Theoretical representation of the $\mathrm{CNT}$ wetting by molten $\mathrm{Al}_{2} \mathrm{O}_{3}$.

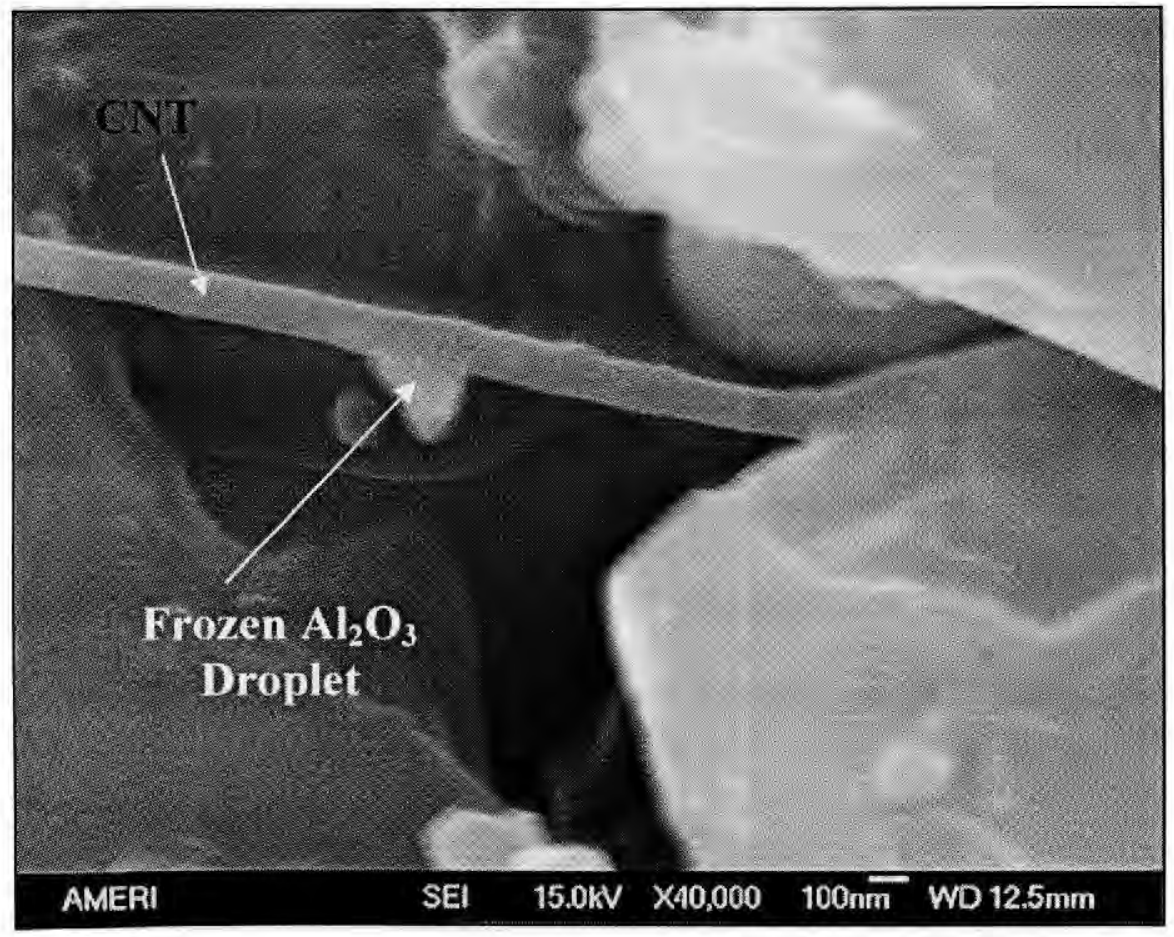

Fig. 4.57: Rapid kinetics of plasma spraying demonstrating freezing of $\mathrm{Al}_{2} \mathrm{O}_{3}$ droplet before surface tension is overcome by gravity. 
Capillarity force $f_{c}$ is defined as [219]:

$$
f_{C}=\frac{\gamma\left(\cos \theta_{1}+\cos \theta_{2}\right) \cdot \Omega}{h_{m}} \quad \text { Equation } 4.12
$$

where $\gamma$ is the surface energy (taken as $\sim 1.59 \mathrm{~J} / \mathrm{m}^{2}$ for $\mathrm{Al}_{2} \mathrm{O}_{3}$ ), and $\Omega$ is surface projection area and $h_{m}$ is the meniscus height. Higher meniscus infers reduced capillarity, but increased surface tension leading to enhanced wetting of CNTs by $\mathrm{Al}_{2} \mathrm{O}_{3}$. Surface tension $T_{S}$ is defined as [197]:

$$
T_{S}=l \cdot \gamma \cdot \cos \alpha
$$

Equation 4.13

where $l$ is the perimeter contact, and $\gamma \cdot \cos \alpha$ is the vertical component of the surface tension, Fig. 4.56. Surface tension is caused by difference in the magnitude of surface forces where difference in forces of adhesion and cohesion results wetting/dewetting of liquid droplets onto substrates.

Theoretical calculations performed on the system are presented in Table 4.10. CNT diameter is assumed to be $70 \mathrm{~nm}$, approximate angles of contact are calculated from the experimental results (SEM micrograph, Fig. 4.43), and various meniscus heights are assumed to theoretically calculate the perimeter contact, and the surface projection area. Meniscus height, perimeter contact and surface projection area are dominating factors, which define the capillary force and surface tension occurring at $\mathrm{Al}_{2} \mathrm{O}_{3}$ coated CNT interface.

Figure 4.58 describes various interactions in assimilating interfacial capillarity and surface tension with respect to meniscus height of $\mathrm{Al}_{2} \mathrm{O}_{3}$ over CNT in differential microstructures. This depends on time and temperature experienced during plasma spraying. As the meniscus height increases, the surface tension of molten $\mathrm{Al}_{2} \mathrm{O}_{3}$ should 
Table 4.10: Theoretical calculations of capillary force and surface tension on $\mathrm{Al}_{2} \mathrm{O}_{3}$-CNT interface (CNT diameter assumed to be $70 \mathrm{~nm}):\left({ }^{*}\right.$ represents calculated value from observed micrograph, Fig. 4.44)

\begin{tabular}{|c|c|c|c|c|c|c|c|c|}
\hline $\begin{array}{c}\text { Meniscus } \\
\text { Height }\left(h_{m}\right) \\
\text { nm }\end{array}$ & $\begin{array}{c}\theta_{1} \\
\text { degrees }\end{array}$ & $\begin{array}{c}\theta_{2} \\
\text { degrees }\end{array}$ & $\begin{array}{c}\alpha \\
\text { degrees }\end{array}$ & $\begin{array}{l}\text { Perimeter } \\
\text { contact, } \\
\text { (l) } \mathrm{nm}\end{array}$ & $\begin{array}{c}\text { Surface } \\
\text { Projection } \\
\text { area }(\Omega) \mathrm{nm}^{2}\end{array}$ & $\begin{array}{c}\text { Surface } \\
\text { Energy, } \\
\left(\gamma=1.59 \mathrm{~J} / \mathrm{m}^{2}\right)\end{array}$ & $\begin{array}{c}\text { Capillary } \\
\text { force }\left(f_{c}\right) n N\end{array}$ & 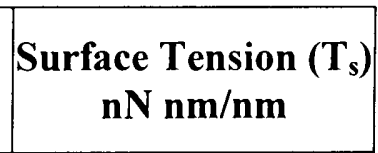 \\
\hline 10 & 16.39 & 13.34 & 64.29 & 30 & 40 & 1.59 & 12.61 & 40.4 \\
\hline 25 & 15.36 & 18.85 & 25.72 & 75 & 90 & 1.59 & 11.32 & 116.3 \\
\hline 30 & 24.20 & 17.86 & 0.00 & 105 & 110 & 1.59 & 9.83 & 167.0 \\
\hline 50 & 14.04 & 15.64 & -38.57 & 150 & 160 & 1.59 & 10.09 & 225.1 \\
\hline $70^{*}$ & 14.87 & 16.32 & -90.00 & 210 & 195 & 1.59 & 8.78 & 236.2 \\
\hline
\end{tabular}




\section{Surface forces on CNT- $\mathrm{Al}_{2} \mathrm{O}_{3}$ interface}

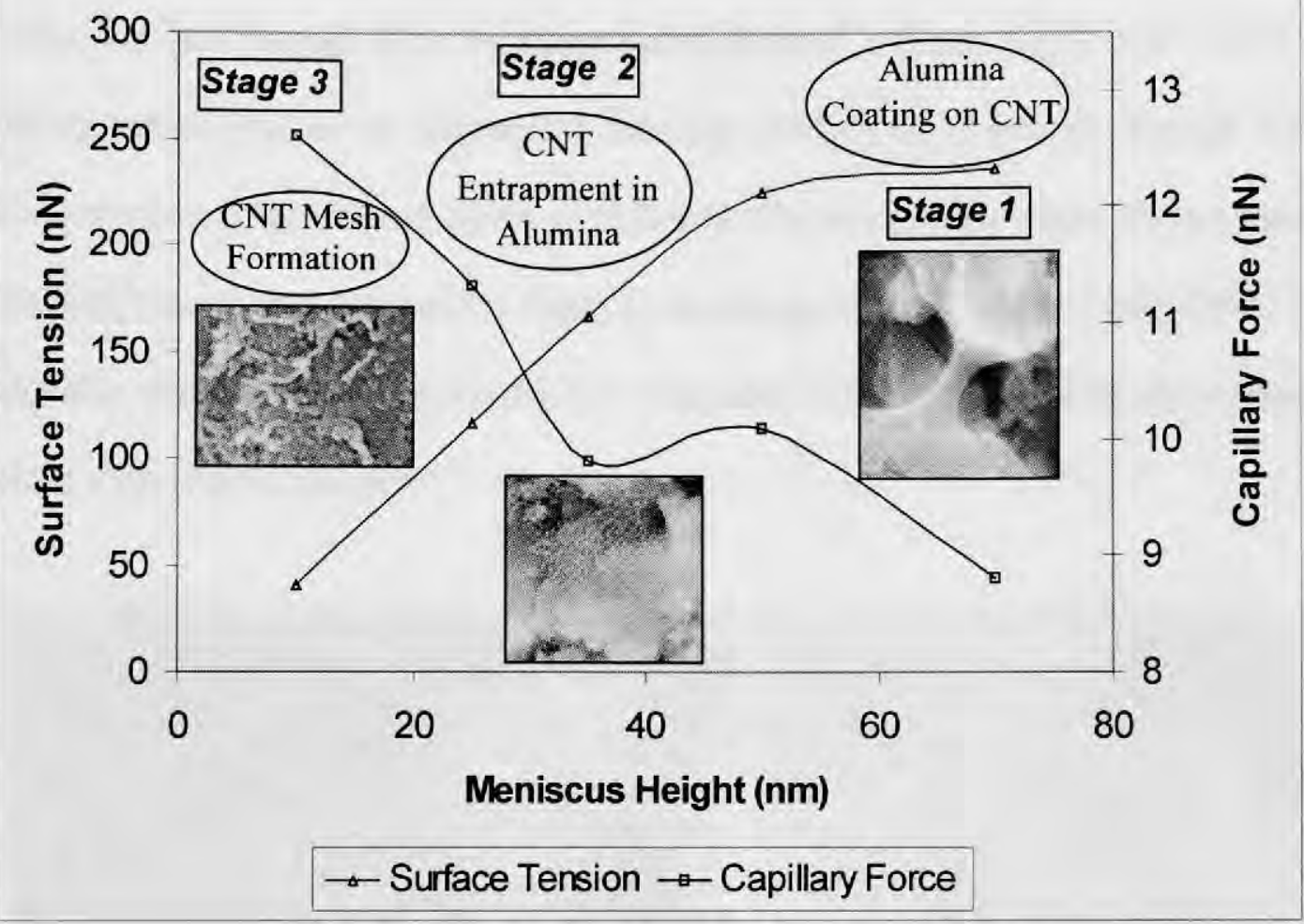

Fig. 4.58: Surface forces on CNT- $\mathrm{Al}_{2} \mathrm{O}_{3}$ interface (CNT diameter assumed $70 \mathrm{~nm}$ ).

suffice its holding against capillarity to seep down. Similarly low meniscus height insinuates ease of holding down molten $\mathrm{Al}_{2} \mathrm{O}_{3}$ rather than allowing it to rise as a coating over CNT. It must be clarified that seeping down of $\mathrm{Al}_{2} \mathrm{O}_{3}$ can occur even at a later stage when enough mass of molten $\mathrm{Al}_{2} \mathrm{O}_{3}$ dragged over $\mathrm{CNT}$ cannot be supported by surface tension. These phenomena can be further split into three stages namely:

i) Stage 1: Surface tension dominated region

ii) Stage 2: Intermixed mode

iii) Stage 3: Capillarity dominated region 
Stage 1 is characterized by occurrence of high surface tension $(\sim 236.2 \mathrm{nN}$ $\mathrm{nm} / \mathrm{nm}$ ), wherein molten $\mathrm{Al}_{2} \mathrm{O}_{3}$ freezes onto CNT to form a thick coating, Fig. 4.59. The process has just enough time to allow quick flow of molten $\mathrm{Al}_{2} \mathrm{O}_{3}$ over CNTs via capillary action, and cause consequent freezing of $\mathrm{Al}_{2} \mathrm{O}_{3}$ as a coating. Though CNTs assist reduction force seeping down of molten $\mathrm{Al}_{2} \mathrm{O}_{3}$ by capillary flow, surface tension force dominates and holds molten $\mathrm{Al}_{2} \mathrm{O}_{3}$ as a coating (upto $40-50 \mathrm{~nm}$ ) over CNT. This bridge-like structure improves the fracture toughness of the nanocomposite by serving as anchors to the adjoining splats.

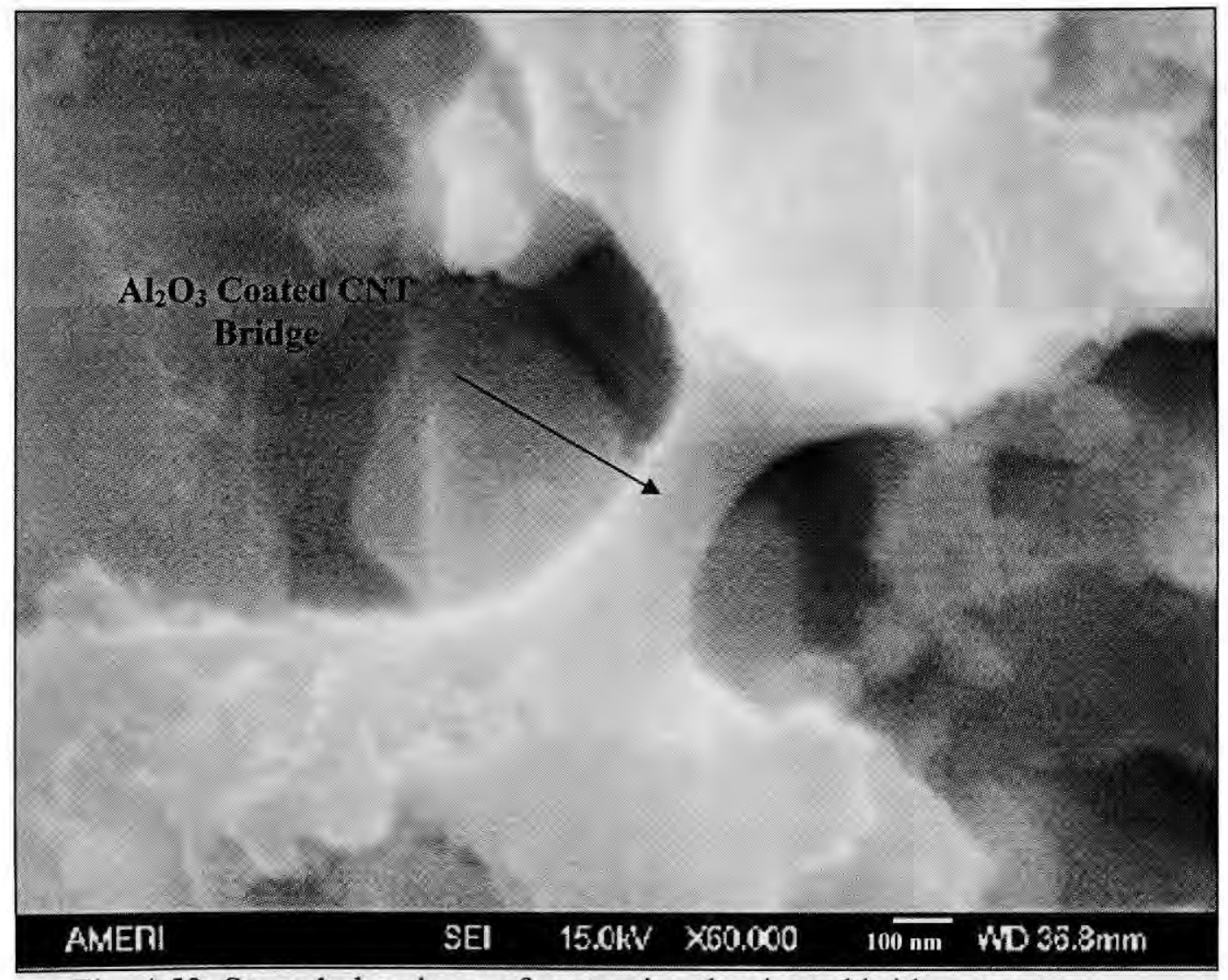

Fig. 4.59: Stage 1 showing surface tension dominated bridge structure. 
Intermediate mode (stage 2) is described by entrapment of CNTs along the solid state sintered $\mathrm{Al}_{2} \mathrm{O}_{3}$ regions, where wetting by $\mathrm{Al}_{2} \mathrm{O}_{3}$ is just occurring, and there is just sufficient time to entrap a few CNTs before they can merge as a mesh. Both capillarity and surface tension of $\mathrm{Al}_{2} \mathrm{O}_{3}$ play a key role in generating the trimodal microstructure, Fig. 4.60. Trimodal microstructure is highly beneficial in enhancing the fracture toughness of nanocomposite, i.e., second phase strengthening by nanoparticles and CNTs, structural strengthening by fully molten regions, energy absorption at partially molten regions, and ductility enhancement by grain sliding of nano particles.

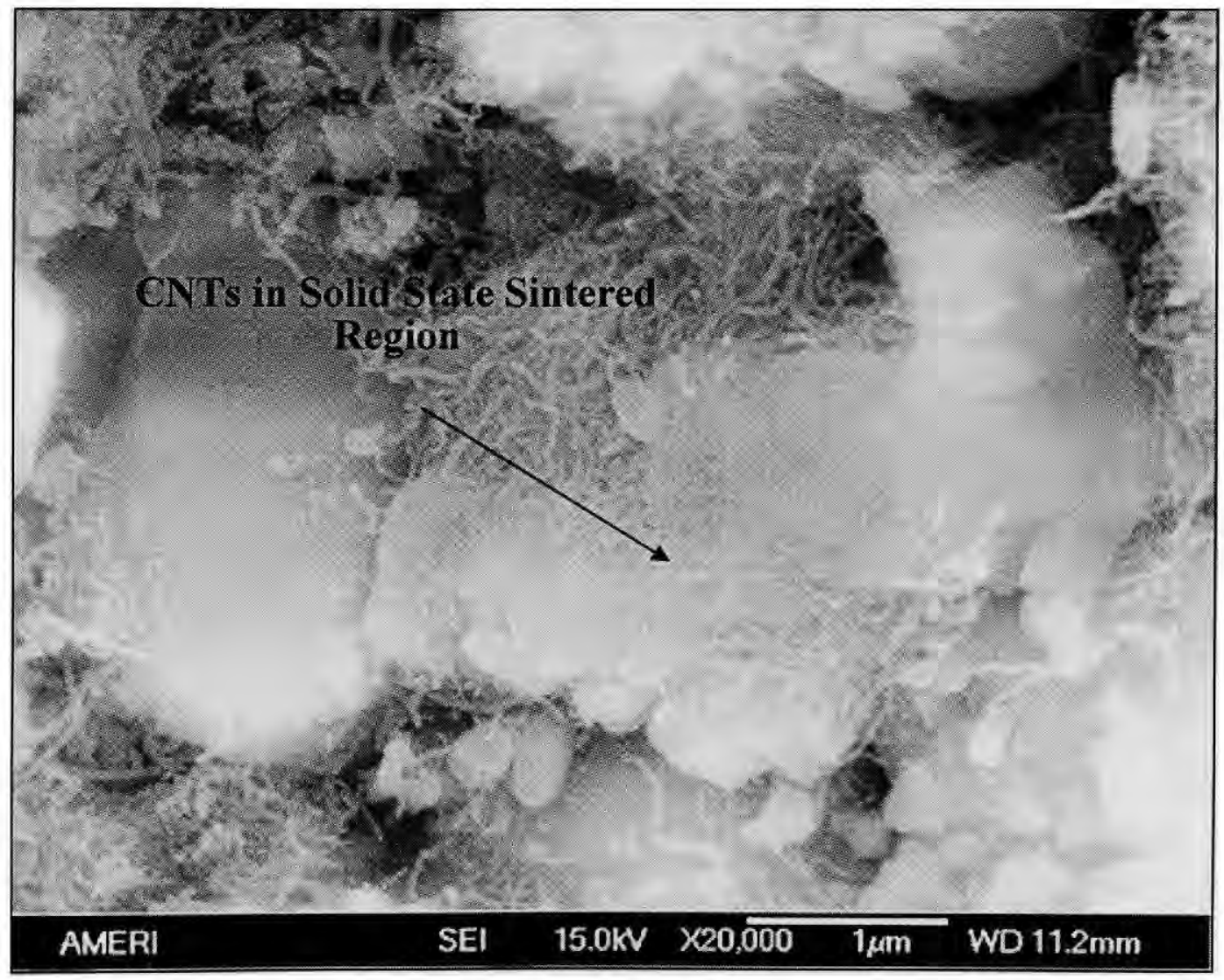

Fig. 4.60: Stage 2 showing CNTs entrapped in $\mathrm{Al}_{2} \mathrm{O}_{3}$. 
Once the molten $\mathrm{Al}_{2} \mathrm{O}_{3}$ flows onto CNT surface, the surface tension of liquid attracts the surrounding $\mathrm{Al}_{2} \mathrm{O}_{3}$-coated $\mathrm{CNT}$ and merges them together. After enough $\mathrm{Al}_{2} \mathrm{O}_{3}$-surfaced CNTs are accumulated altogether, it becomes difficult to hold molten alumina by surface tension alone. Owing to the increased volume of surrounding liquid (molten $\mathrm{Al}_{2} \mathrm{O}_{3}$ ), the capillarity reduces the surface tension by its seeping out molten $\mathrm{Al}_{2} \mathrm{O}_{3}$ and leaving $\mathrm{CNT}$ structure as a mesh, Fig. 4.61 (stage 3). This mesh has a very thin layer (few nanometers) of molten $\mathrm{Al}_{2} \mathrm{O}_{3}$, which in true sense is reinforcement by flow of $\mathrm{Al}_{2} \mathrm{O}_{3}$ ceramic into intricate surface of CNT. Thickening and smoothening of CNTs is visible in Fig. 4.61.

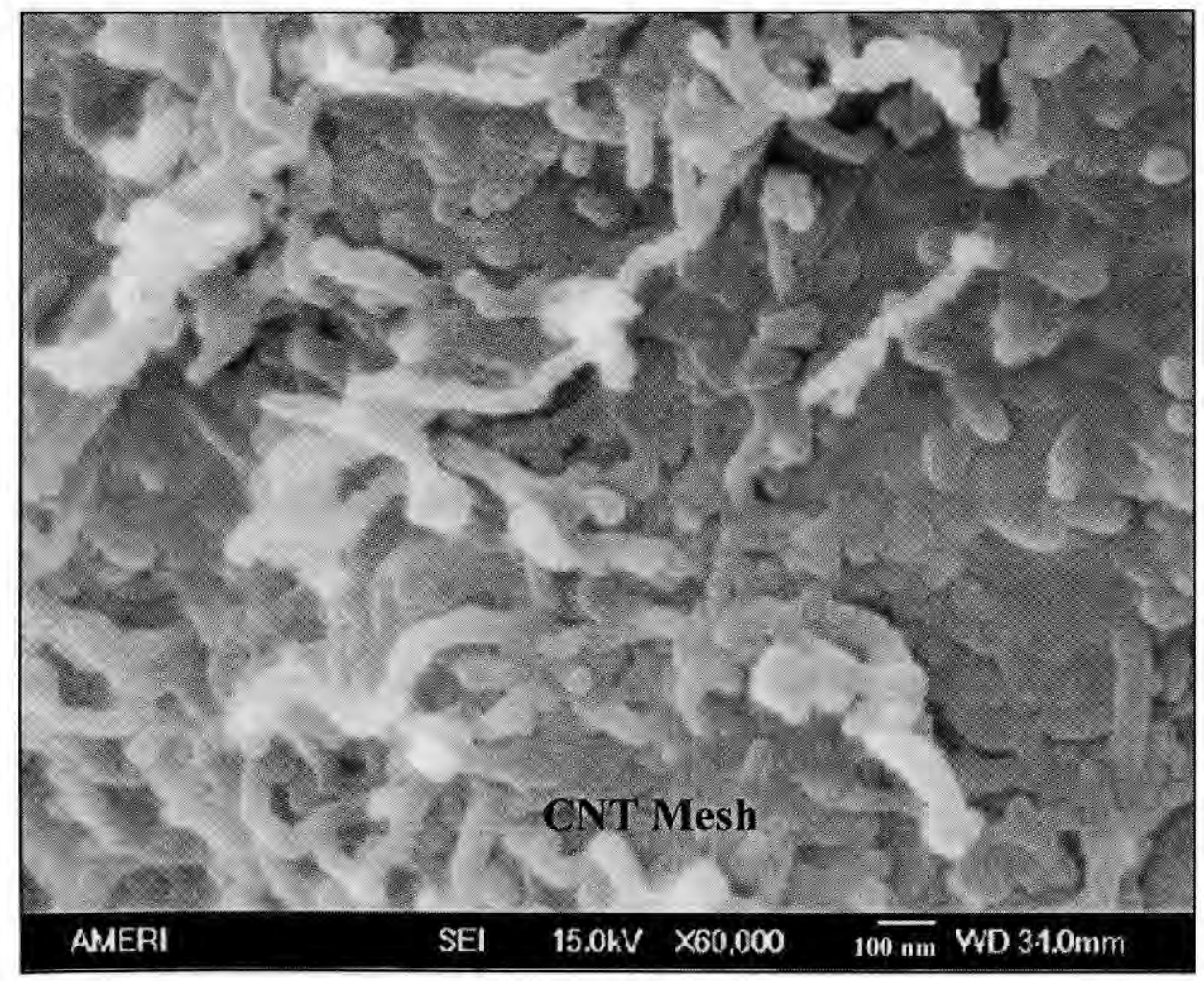

Fig. 4.61: Stage 3 showing capillarity dominated CNT-mesh. 
Hence, excellent wettability of $\mathrm{Al}_{2} \mathrm{O}_{3}$ on $\mathrm{CNT}$ surface is observed via formation of $\mathrm{Al}_{2} \mathrm{O}_{3}$ coated CNT, solid state sintering and CNT mesh formation (Fig. 4.56-4.61). Rapid solidification kinetics, inherent to plasma spraying, might be reasoned to attribute enhanced wettability observed in the current work. Nucleation frequency $\left(I_{\nu}\right)$ of $\mathrm{Al}_{2} \mathrm{O}_{3}$ on CNT surface at a temperature $(T)$ can be expressed by eq. 4.14 as [224]:

$$
I_{v}=K_{v} \exp \left(-\frac{\Delta G^{*} f(\theta)}{k T}\right)
$$

where $K_{v}$ is kinetic parameter, $\theta$ is the wetting angle, and $\Delta G^{*}$ is the excess free energy of the critical nucleus, and $k$ is the Boltzmann constant. Since rapid solidification offers enhanced excess free energy for nucleation, it must be balanced by reduction in the wetting factor $f(\theta)$. The wetting factor expression [225] $\left\{\mathrm{f}(\theta)=(2+\cos \theta)(1-\cos \theta)^{2} / 4\right\}$ further clarifies reduction of wetting angle (and enhanced wettability). Moreover, heterogeneous nucleation reduces the excess energy barrier of nucleation [225], allowing rapid solidification of $\mathrm{Al}_{2} \mathrm{O}_{3}$ by wetting CNT surface.

Wetting, therefore immediately raises the question of secondary phase generation at the $\mathrm{CNT}-\mathrm{Al}_{2} \mathrm{O}_{3}$ interface. In order to understand the fundamental thermodynamic predictions, FactSage thermochemistry software is used to construct phase diagram of AlO-C system (Fig. 4.62). $\mathrm{Al}_{2} \mathrm{O}_{3}$ and $\mathrm{C}$ emerge as the only stable phases present in the current systems (A4C-B, A4C-SD, and A8C-SD). Thermodynamics calculations convey very low activity $\left(\sim 6.8889 \times 10^{-19}\right.$ at $\left.2200 \mathrm{~K}\right)$ of $\mathrm{Al}_{4} \mathrm{C}_{3}$ even near the melting point of $\mathrm{Al}_{2} \mathrm{O}_{3}$, and on the other hand, $\mathrm{CO}$ and $\mathrm{CO}_{2}$ product gases will tend to destabilize the 
interface. Consequently, there is no stable reaction product of $\mathrm{Al}_{2} \mathrm{O}_{3}$ reaction with $\mathrm{C}$, eq. 4.15.

$$
\begin{aligned}
& 2 \mathrm{Al}_{2} \mathrm{O}_{3}+6 \mathrm{C} \rightarrow \mathrm{Al}_{4} \mathrm{C}_{3}+3 \mathrm{CO}_{2} \\
& \Delta \mathrm{G}=+700.2 \mathrm{KJ}(\text { at } 2200 \mathrm{~K}) \\
& \Delta \mathrm{G}=+558.4 \mathrm{KJ}(\text { at } 2500 \mathrm{~K})
\end{aligned}
$$

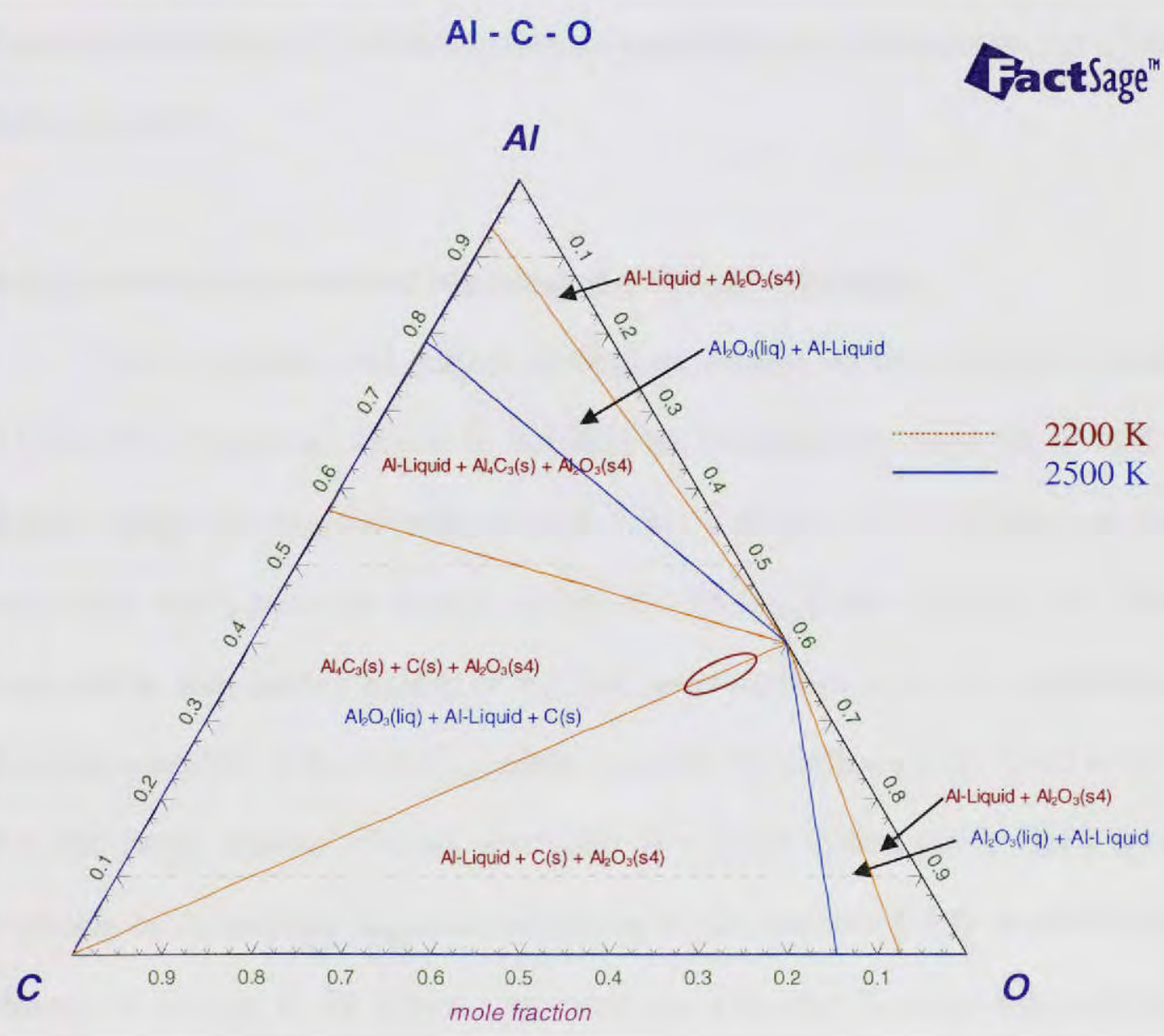

Fig. 4.62: Phase stability diagram calculated from FactSage software [226]. Activity of $\mathrm{Al}_{2} \mathrm{O}_{3}=1$, activity of carbon $=1$, and activity of $\mathrm{Al}_{4} \mathrm{C}_{3}(\mathrm{~s})=1.7429 \times 10^{-18}$. 
Hence, formation of secondary phases such as carbides is not preferred in the AlO-C system. In addition, absence of prism planes in CNT also restrict free $\mathrm{C}$ bonds being available for bonding with $\mathrm{Al}$ and $\mathrm{O}$. Surface wetting of CNTs by $\mathrm{Al}_{2} \mathrm{O}_{3}$ is therefore not influenced by presence of any secondary phases, and can be extracted as direct interacting interface. After the interface is defined, ab-initio computational modeling can provide atomic interaction at molecular level. Visualization of interfacial atoms can evolve understanding of interfacial reactions enhancing the fracture toughness of ceramic nanocomposite.

\subsection{Ab-initio Computational Modeling of $\mathrm{CNT}-\mathrm{Al}_{2} \mathrm{O}_{3}$ Interface}

Since capillarity and surface tension are defined by the interfacial reaction of $\mathrm{Al}_{2} \mathrm{O}_{3}-\mathrm{CNT}$, it becomes critical to visualize the molecular phenomenon at such level. Surface energy of oxygen terminated $\mathrm{Al}_{2} \mathrm{O}_{3}$ is in the range $4.45-10.83 \mathrm{~J} / \mathrm{m}^{2}$, whereas $\mathrm{Al}$ terminated $\mathrm{Al}_{2} \mathrm{O}_{3}$ possesses surface energy of $1.59 \mathrm{~J} / \mathrm{m}^{2}$ [196]. Though CNT surface is much stable with surface energy of $0.2 \mathrm{~J} / \mathrm{m}^{2}$, nano surfaces often are understudied and depict non-intuitive behavior. Al-graphite can drain the surface energy down in the 0.02 $0.4 \mathrm{~J} / \mathrm{m}^{2}$ range, making a strong possibility of a stable system [194]. Ooi's molecular modeling of Al-graphite depicted no bonding at the interface [194]. In addition to the absence of oxygen in the system, representation with specific cross-sectional plane in demonstrating Al-C interface, overall bonding at the interface was absent.

In the current $\mathrm{Al}_{2} \mathrm{O}_{3}-\mathrm{CNT}$ system, stable $1 \mathrm{x} 1 \mathrm{x} 1 \alpha-\mathrm{Al} 2 \mathrm{O} 3(\mathrm{a}=4.76 \AA \mathrm{x} \mathrm{b}=4.76 \AA \mathrm{x}$ $\left.c=12.99 \AA, \alpha=90^{\circ}, \beta=90^{\circ}, \gamma=120^{\circ}\right)$ crystal is interfaced with $2 \times 2 \times 2$ CNT surface $(a=2.46$ $\AA, b=2.46 \AA, c=6.71 \AA, \alpha=90^{\circ}, \beta=90^{\circ}, \gamma=120^{\circ}$ ), Fig. 4.63. Since Al-terminated $\mathrm{Al}_{2} \mathrm{O}_{3}$ 
shows a more stable interface, Al-terminated $\mathrm{Al}_{2} \mathrm{O}_{3}$ crystal is considered for modeling the interface. Schematic of atomic positions of $\mathrm{Al}_{2} \mathrm{O}_{3}$-CNT system is presented in Fig. 4.63, which define the system considered for atomistic simulation. Ab-initio SIESTA 1.3 computational modeling details are provided in section 3.6. Conjugate gradient optimization of atomic positions provides equilibrium positions of the resulting structure. This consequently provides electronic density surfaces of various layers that can be plotted to understand the interactions occurring near/at $\mathrm{CNT}-\mathrm{Al}_{2} \mathrm{O}_{3}$ interface. Planes $\mathrm{A}$, $\mathrm{B}, \mathrm{C}$ and $\mathrm{D}$ are considered to describe various interactions occurring at various levels of influence from the interface.

Plane C

$\left(\mathrm{Al}_{2} \mathrm{O}_{3}\right.$ Interfacial Layer)

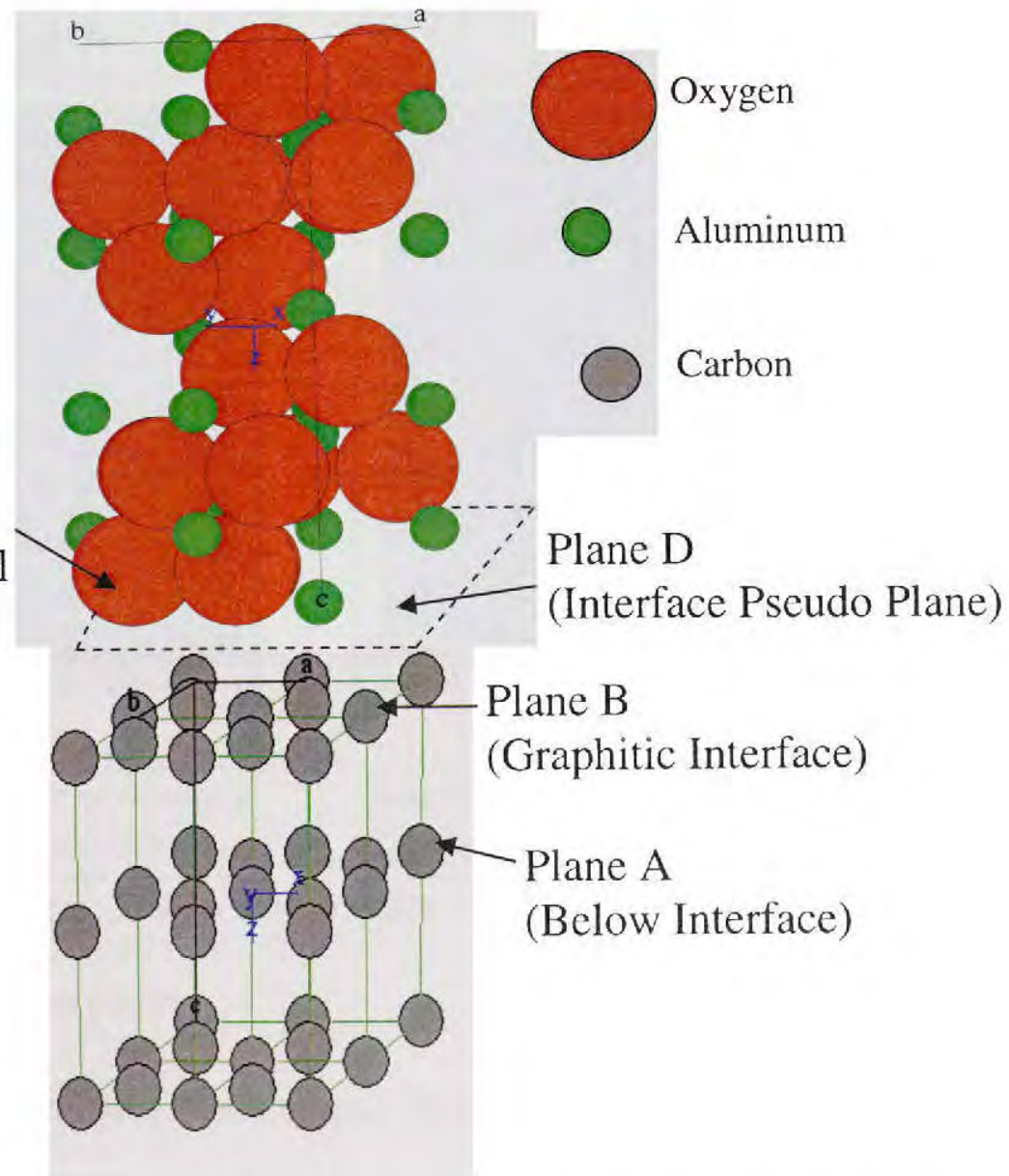

Fig. 4.63: $\mathrm{Al}_{2} \mathrm{O}_{3}$-CNT system defined in ab-initio computational modeling. 
The iso-surface contours for unaffected graphite (0001) layer (plane A) are presented in Fig. 4.64a. This shows the periodic and regular energy contours between the carbon atoms. As the interface approaches, the interference from the $\mathrm{Al}_{2} \mathrm{O}_{3}$ surface on the graphite layer is depicted by the distortion of the periodic energy contours, Fig. 4.64b. Influence from the surface atoms of $\mathrm{Al}_{2} \mathrm{O}_{3}$ crystal depicts clustering of electron cloud between aluminum and carbon along the interface. Oxygen terminated $\mathrm{Al}_{2} \mathrm{O}_{3}$ surface might further destabilize the interface by formation of $\mathrm{CO}$ or $\mathrm{CO}_{2}$. Thermodynamics of the reaction presented as eq. 4.15 clearly obviates the necessity of considering this reaction.

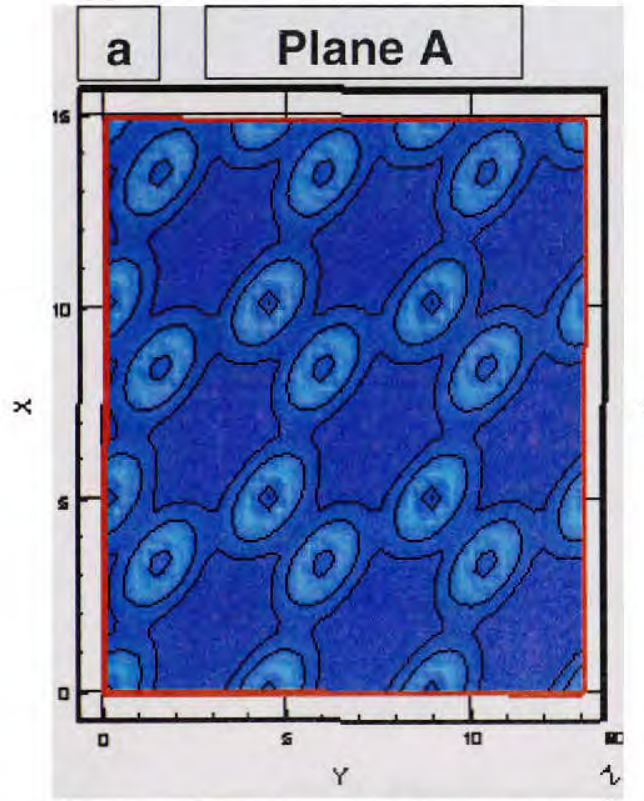

Periodic and regular iso-surface contours of carbon layer
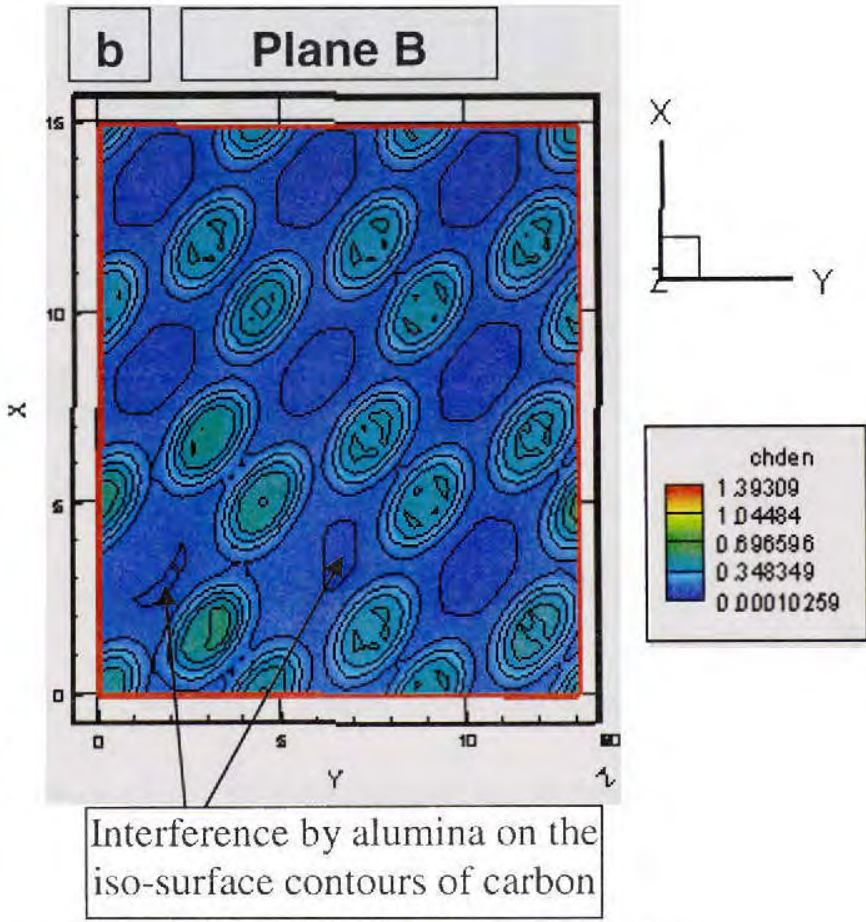

Fig. 4.64: Isosurface contours of graphite layers at a) uninfluenced planes, and b) near interface plane.

Aluminum terminated $\mathrm{Al}_{2} \mathrm{O}_{3}$ crystal illustrates presence of three aluminum atoms at the interface (Plane C), Fig. 4.65a, which further insinuates the contribution of 
aluminum atoms in distorting the energy contours at the interface (plane D), presented in Fig. 4.65b. Pseudo bonding over the aluminum atoms (in the $\mathrm{Al}_{2} \mathrm{O}_{3}$ crystal) is implying pseudo bonding with carbon. Since high polarity indicates strong metallic bonding in aluminum [194], distorted iso-surface contours confirm the pseudo metallic bond at $\mathrm{Al}_{2} \mathrm{O}_{3}-\mathrm{CNT}$ interface. On the Al-terminated surface, weak binding energy with silver
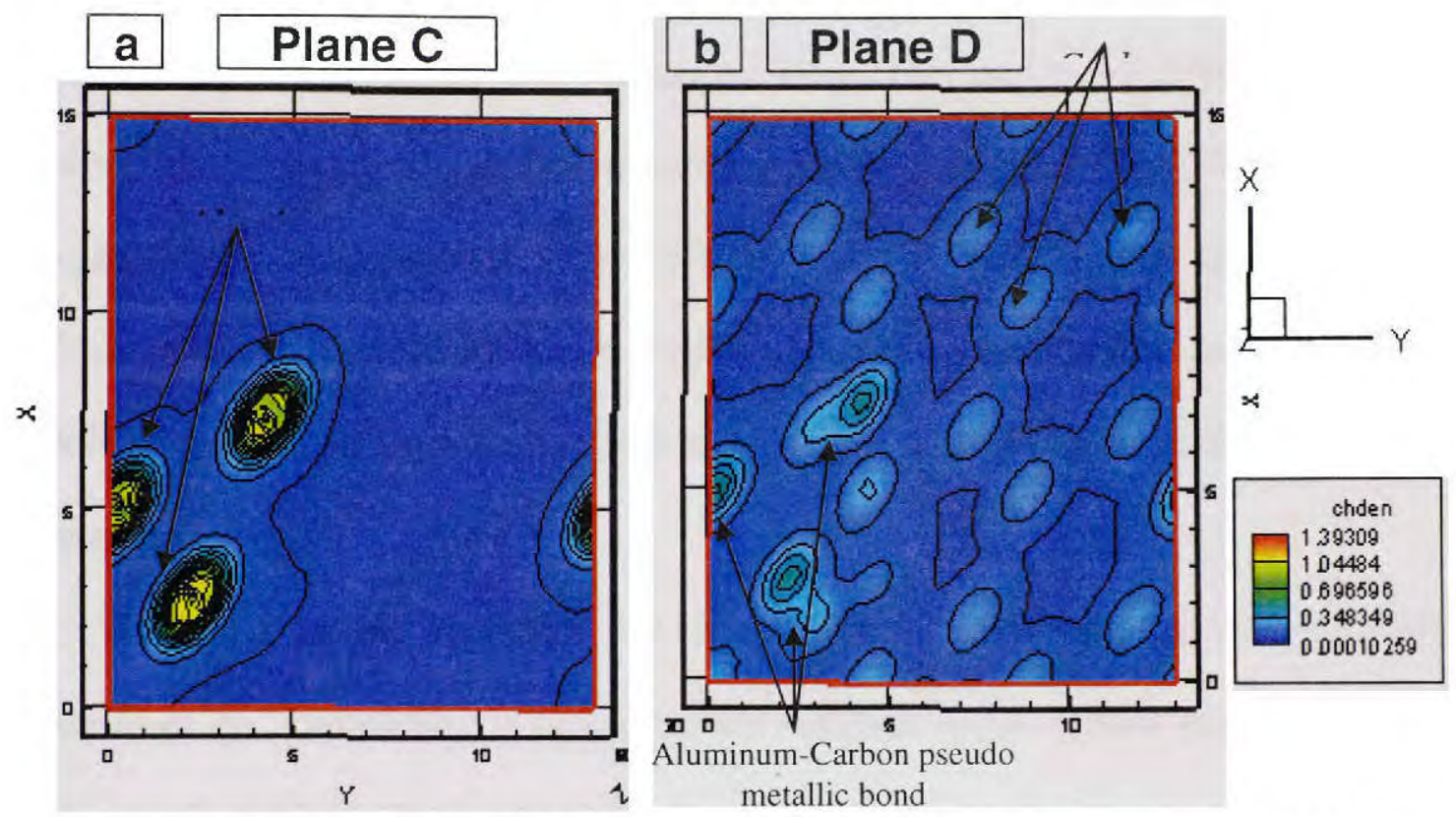

Fig. 4.65: Isosurface of a) aluminum surface-terminated alumina crystal, b)

aluminum-carbon pseudo metallic bond interference at alumina-CNT interface.

$(\sim 0.5 \mathrm{eV})$ and low activation barrier $(\sim 0.25 \mathrm{eV})$ might allow rapid diffusion and bonding on this surface [227]. Direct evidence from molecular modeling result clearly shows the possibility of enhanced interfacial bonding between $\mathrm{Al}$ and $\mathrm{C}$. Enhanced interfacial strength of $\mathrm{Al}_{2} \mathrm{O}_{3}$-CNT system will provide enhanced fracture toughening. It makes sense because crystal with high surface energy will try to adhere to a new surface in order to minimize its overall energy. 
Some experimental data already demonstrates the stability of Al-C interface (energy $\sim 0.02-0.4 \mathrm{~J} / \mathrm{m}^{2}$ ) in comparison to self-existing $\mathrm{Al}_{2} \mathrm{O}_{3}$ or graphite crystals [194]. It is quite viable that partial bonding at interface interconnects the CNTs by introducing humps and encouraging strong wettability. Moreover, the unconnected region acts as energy sink during impact to further enhance the interfacial strength. Though these molecular simulations consider $\mathrm{Al}_{2} \mathrm{O}_{3}$-graphite interface, this model closely mimics the surface properties of $\mathrm{Al}_{2} \mathrm{O}_{3}-\mathrm{CNT}$ interface. This combination of interfacial linking abridges the gap that had existed in describing the wettability of the CNTs with $\mathrm{Al}_{2} \mathrm{O}_{3}$. Consideration of bulk structure, gravity effects, and rapid solidification can further alter the interpretations from the molecular modeling studies. An exhaustive modeling of this work is recommended for computational researchers in describing the surface interaction more effectively.

Interfacial atomic interaction can be scaled through interpretation of load transfer at interfaces via formulating i) contact points between $\mathrm{Al}_{2} \mathrm{O}_{3}$ surface coating the CNT surfaces and ii) $\mathrm{Al}_{2} \mathrm{O}_{3}-\mathrm{Al}_{2} \mathrm{O}_{3}$ splat interface, which completely describes stress transfer between $\mathrm{Al}_{2} \mathrm{O}_{3}$ coated CNTs and $\mathrm{Al}_{2} \mathrm{O}_{3}$ matrix. Since cohesion is stronger in similar adjoining materials $\left(\mathrm{Al}_{2} \mathrm{O}_{3}-\mathrm{Al}_{2} \mathrm{O}_{3}\right)$, the weaker interface $\left(\mathrm{Al}_{2} \mathrm{O}_{3}-\mathrm{CNT}\right.$ interface) is elucidated herewith. Stress distribution along $\mathrm{Al}_{2} \mathrm{O}_{3}-\mathrm{CNT}$ interface can be modeled by considering longitudinal stress of $\sigma_{f}$ occurring within a distance of $d x$, balanced by interfacial shearing, $\tau_{i}$, as shown in Fig. 4.66 using eq. 4.16 as:

$$
2 \pi r t\left(\sigma_{f}+d \sigma_{f}\right)-2 \pi r t \sigma_{f}-2 \pi r \tau \cdot d x=0 \quad \text { Equation } 4.16
$$

which simplifies to 
$\frac{d \sigma_{f}}{d x}=\frac{\tau}{t}$

where $t$ is the wall thickness of the CNT.

Wettability influences the transition from interfacial to longitudinal stresses. Thereby shearing between $\mathrm{Al}_{2} \mathrm{O}_{3}-\mathrm{CNT}$ interface reinforces energy absorption resulting in enhanced toughening.

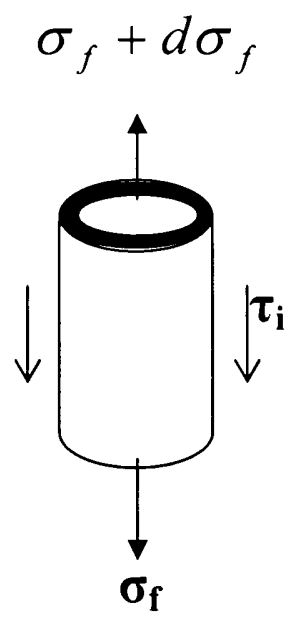

Fig. 4.66: Interfacial Shear at $\mathrm{Al}_{2} \mathrm{O}_{3}-\mathrm{CNT}$ interface.

Dominance of surface tension in conjunction with rapid freezing (section 4.7) can evolve fascinating toughening junctions, as seen in Fig. 4.67. To reduce its surface energy, molten $\mathrm{Al}_{2} \mathrm{O}_{3}$ accumulates coated CNTs as ropes. Incomplete termination of process can result formation of Y-junction ropes, which act as toughening anchors in the matrix. Multi directional load bearing capability (shear and axial strength, eqs. 4.6 and 4.17) and flexibility (bending stiffness, eq. 4.5) at other end of the junction-ropes can improve the fracture toughness of the ceramic nanocomposite. 
Though various mechanisms interplay in deciding the fracture toughness, representative mechanical properties of generated structure can also be mapped from its elastic modulus. Next section deals with the use of nanoindentation technique in evaluating modulus of the plasma sprayed coatings.

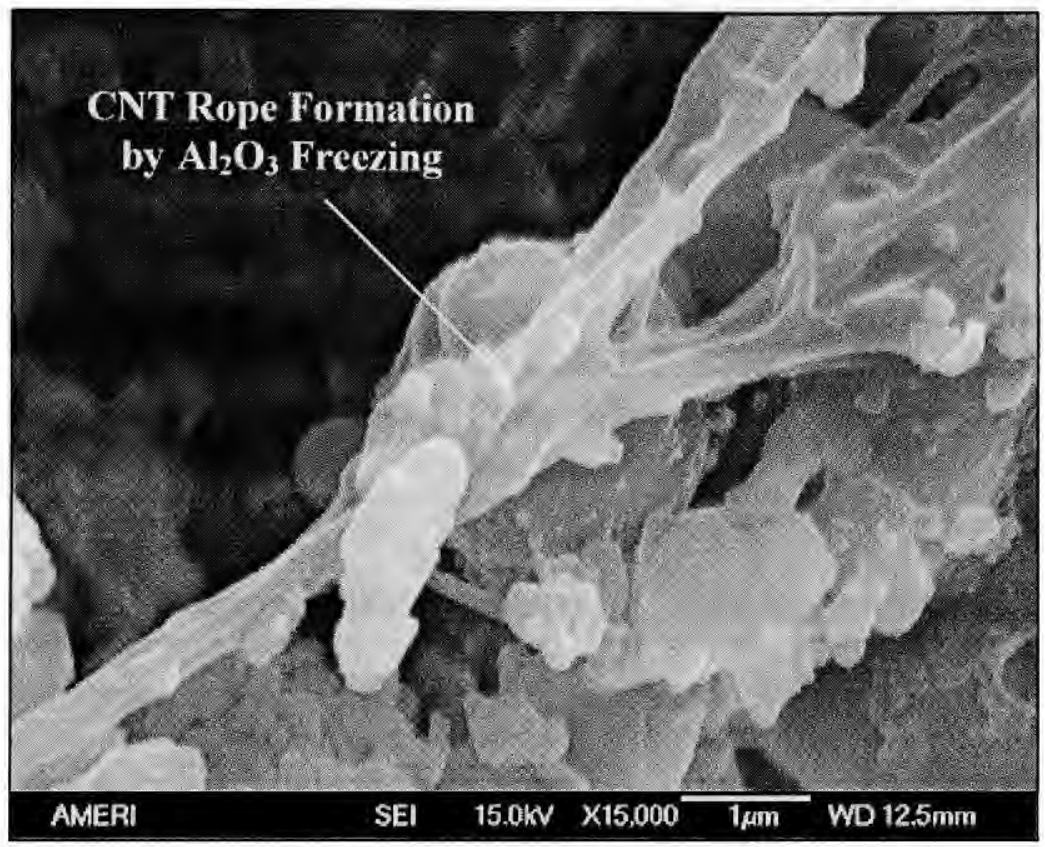

Fig. 4.67: Dominance of surface tension in forming CNT Y-junction ropes,

\subsection{Nanomechanical Modulus Mapping of Plasma Sprayed Coatings}

Nanomechanical dynamic analyzer provides unprecedented characterization of materials, such as modulus mapping of the plasma sprayed coatings over an area (to render the stiffness associated with the content and distribution of CNTs). Nanoindenter tip oscillates with small forces and rasters along the surface to monitors the displacement and phase lag arising from material's response. Though surface asperities also influence the modulus of the coating, uniformity of modulus can insinuate the dispersion of CNTs in the coatings. Surface profile and corresponding modulus mapping is correlated with 
the distribution of modulus values to associate the overall range of modulus pertaining to the coating. Instead of complex modulus, which involves the loss modulus, storage modulus is taken as direct representative of the Young's modulus considering the nonplastic fracturing of ceramics. Area of $4 \mu \mathrm{m} \times 4 \mu \mathrm{m}$ is selected for modulus mapping in order to scan wide enough section in commenting the role of CNT content and dispersion in contributing enhanced modulus of the plasma sprayed coatings.

\subsubsection{Modulus Mapping of A-SD Coating}

Surface profile of A-SD coating, Fig. 4.68a, is showing fine surface undulations and nano scratches. Corresponding modulus mapping, Fig. $4.68 \mathrm{~b}$, show uniform structure with modulus average of approximately around $210 \mathrm{GPa}$ as observed in the color spread. Blue streaks correspond to the scanning limit of the SPM (Scanning Probe Microscopy) and edge effects arising from sharp corners. Neglecting those features, distribution of the modulus along distance, Fig. 4.68c, and histogram of storage modulus, Fig. 4.68d, provide average storage modulus around $210 \mathrm{GPa}$. Lower modulus values are typical to the plasma sprayed coating, which are attributed to layered structure and porosity, which is inherent to plasma sprayed structure. These modulus values can be taken as base values for comparison with modulus mapping in presence of CNTs.

\subsubsection{Modulus Mapping of A4C-B Coating}

Topography of A4C-B coating is observed in Fig. 4.69a, which shows uniform structure. Modulus mapping of A4C-B coating, Fig. 4.69b, shows storage modulus in the 
range of 200-300 $\mathrm{GPa}$, with some regions reaching values upto $650 \mathrm{GPa}$, Fig. 4.69c. Localized high value of modulus is attributed to the presence of CNTs at such locations. Modulus mapping histogram shows average modulus of $\sim 250 \mathrm{GPa}$, Fig. 4.69d. Shift of average modulus in A4C-B to higher vale (when compared to A-SD coating) is attributed to the presence of CNTs in the structure.

\subsubsection{Modulus Mapping of A4C-SD Coating}

In the uniform area of the topographical image shown in Fig. 4.70a, average modulus value is $\sim 300 \mathrm{GPa}$ in the mapped storage modulus (Fig. 4.70b). Distribution of modulus along the distance is presented in Fig. 4.70c. Reduced modulus values $(<250$ $\mathrm{GPa}$ ) is arising from the valleys present on the surface. Dominating regions of modulus appear bimodally at $250 \mathrm{GPa}$ and $350 \mathrm{GPa}$, as presented in the histogram, Fig. $4.70 \mathrm{~d}$. Modulus values average at $\sim 300 \mathrm{GPa}$, which is higher than modulus of A4C-B coatings $(\sim 250 \mathrm{GPa})$. Increase in the modulus is attributed to uniform CNT dispersion in the matrix. Bimodal modulus distribution can be attributed to the presence of FM and PM regions.

Reduced modulus of $\sim 250 \mathrm{GPa}$ can be reasoned to presence of high solid state sintered region where particles are consolidated by surface sintering. Increased modulus of $\sim 350 \mathrm{GPa}$ can be reasoned to FM region which is densified by surface melting and resolidification (along with entrapped CNTs). Hence the role of CNT dispersion (in A4CSD coating) is lucidly detailed by modulus mapping in comparison to that of $\mathrm{A} 4 \mathrm{C}-\mathrm{B}$ coating. 


\section{a}

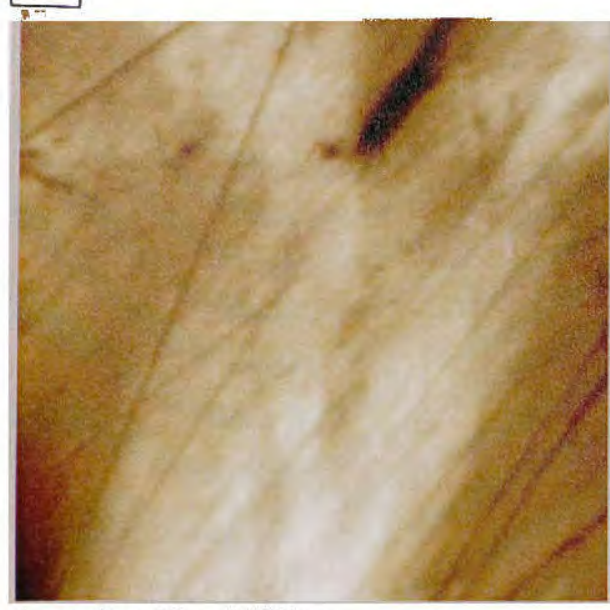

Image Scan Size: $4.000 \mu \mathrm{m}$ $\mathrm{nm}$

40.1

20.0

0.0

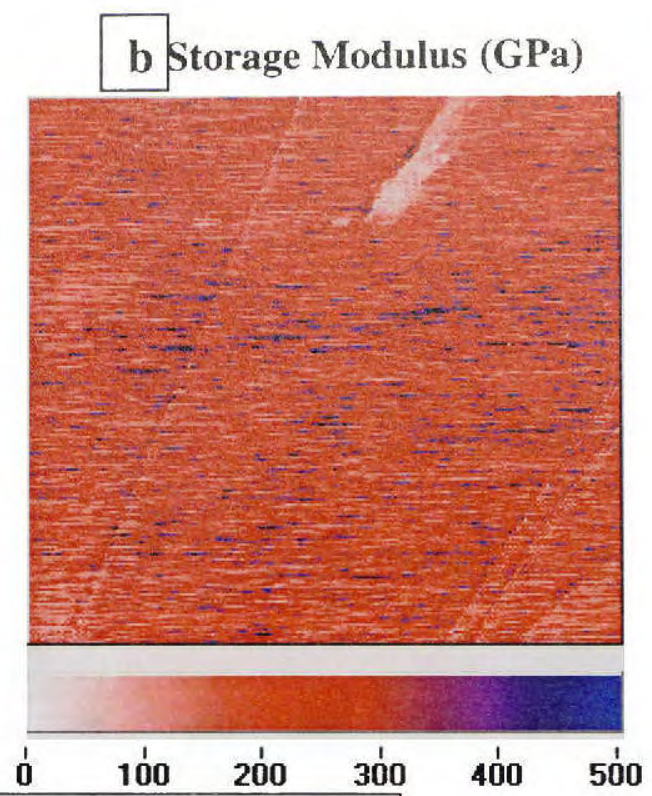

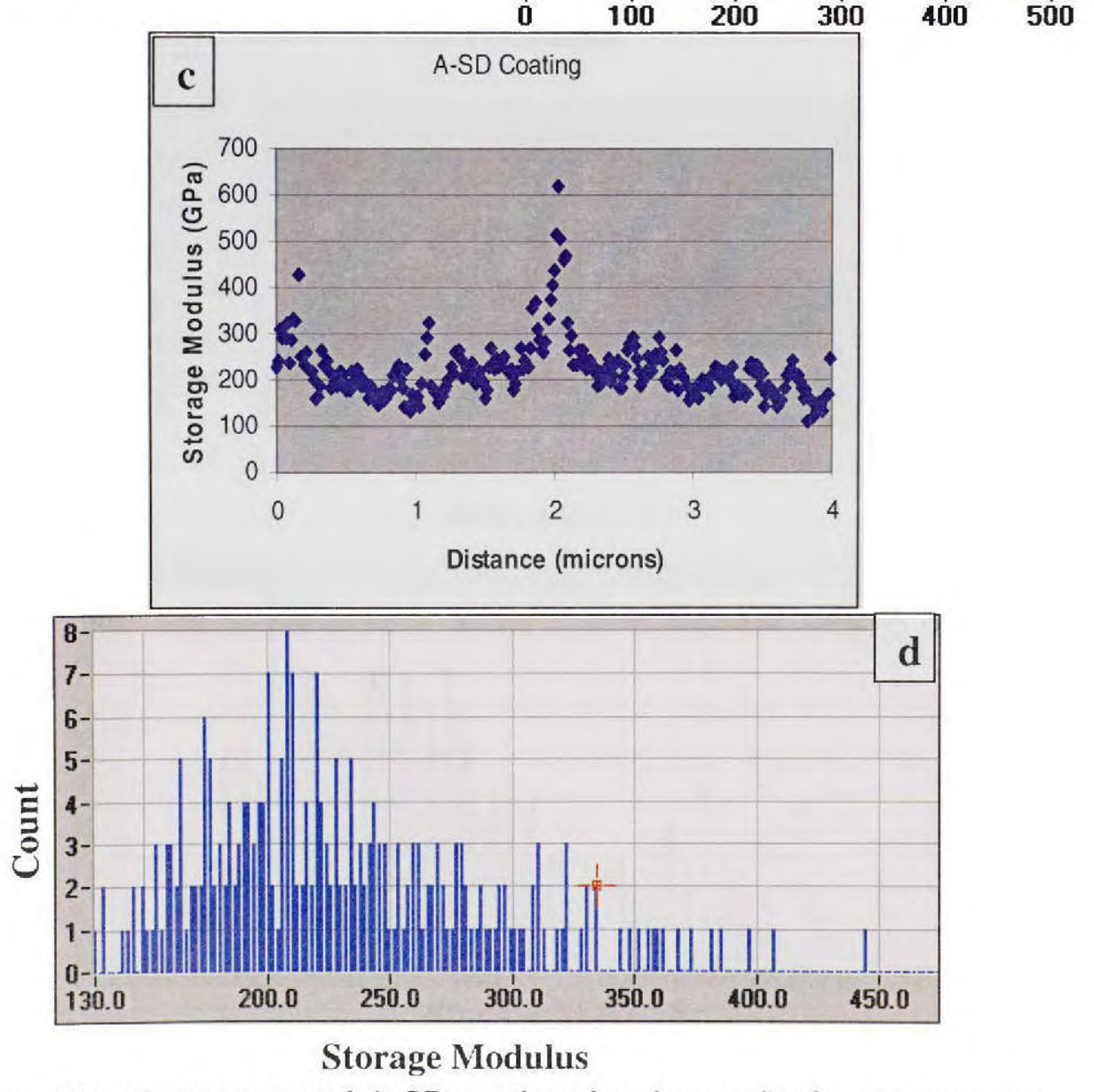

Fig. 4.68: Plasma sprayed A-SD coating showing, a) Surface topography,

b) Modulus map, c) Line scan distribution of storage modulus with distance, and d) Histogram of modulus distribution. 

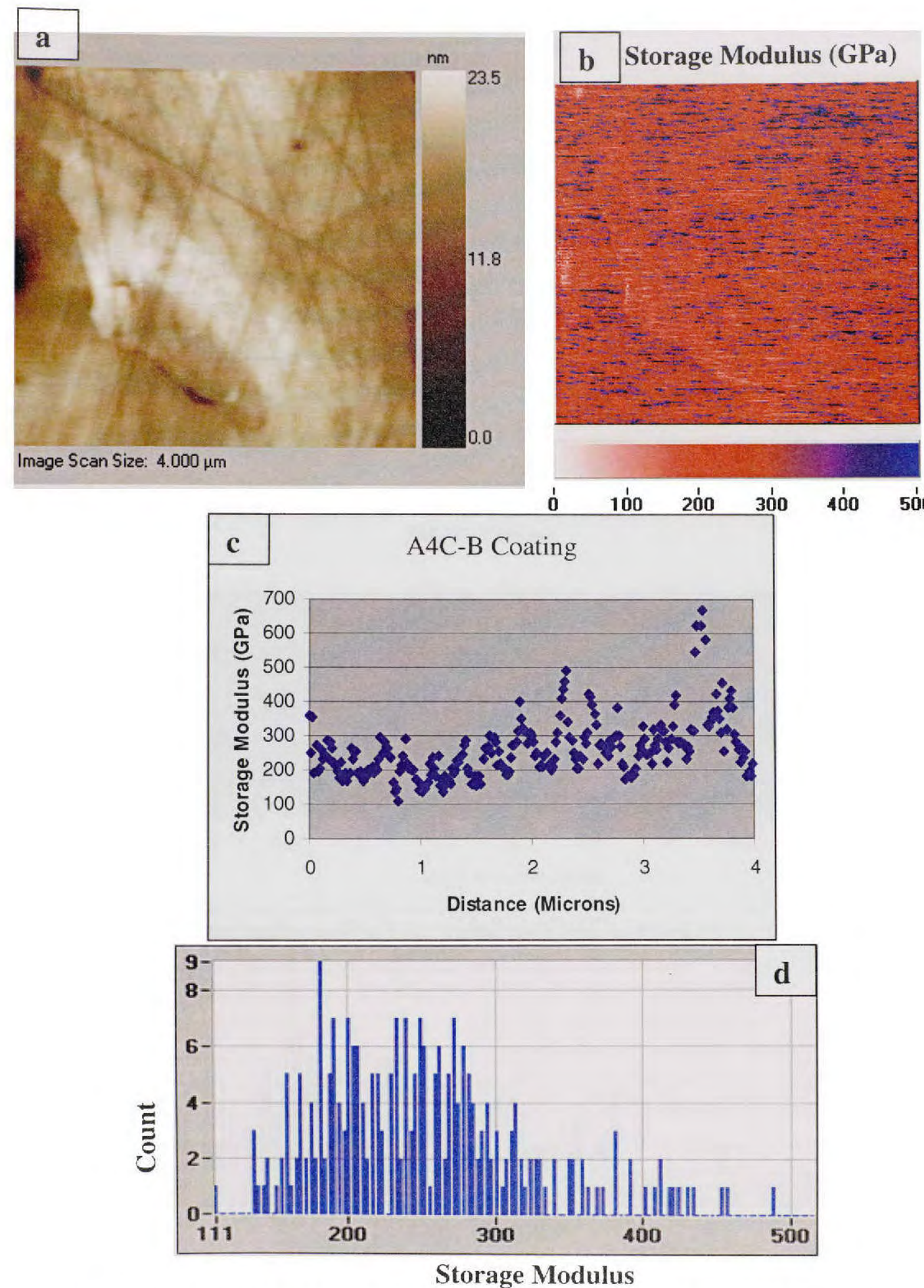

Fig. 4.69: Plasma sprayed A4C-B coating showing, a) Surface topography, b) Modulus mapping, c) Line scan distribution of storage modulus with distance, and d) Histogram of modulus distribution. 

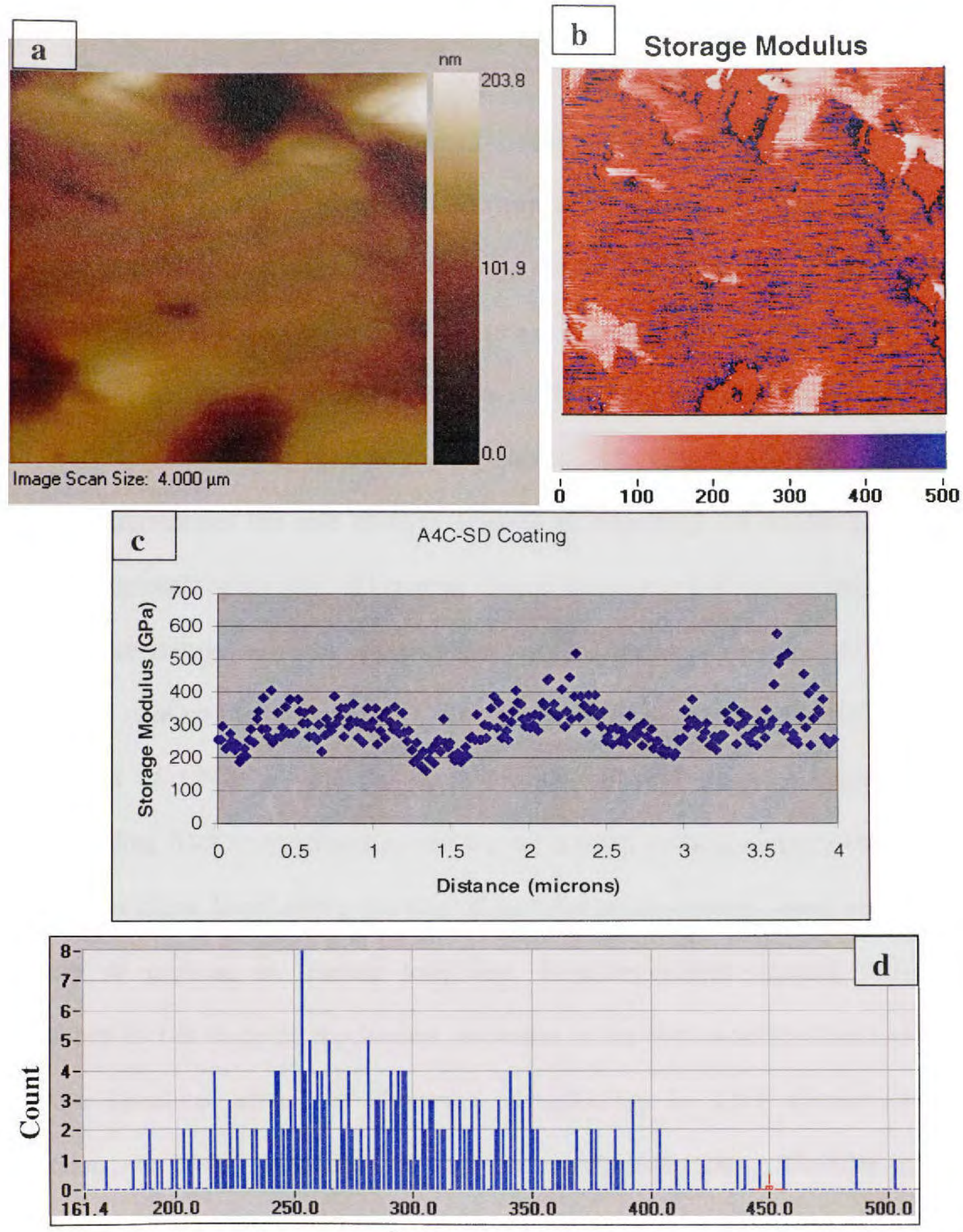

\section{Storage Modulus}

Fig. 4.70: Plasma sprayed A4C-SD coating showing, a) Surface topography, b) Modulus mapping, c) Distribution of storage modulus with distance, and d) Histogram of modulus distribution. 


\subsubsection{Modulus Mapping of A8C-SD Coating}

Topography of plasma sprayed A8C-SD coating appears rough, Fig. 4.71a, but modulus mapping shows enhanced overall modulus, Fig. 4.71b. Dispersion of modulus line scan, Fig. $4.70 \mathrm{c}$ shows modulus in the range of $200-500 \mathrm{GPa}$. Here again, bimodal modulus peaks are observed at $\sim 325 \mathrm{GPa}$ and $\sim 420 \mathrm{GPa}$ in Fig. 4.71d. Increase in the modulus is attributed to enhanced CNT content in the coating. Dispersion of CNTs in the solid state sintered region is resulting modulus $\sim 325 \mathrm{GPa}$, whereas CNTs reinforcing the fully molten region further increase the modulus to $\sim 420 \mathrm{GPa}$. Hence, modulus mapping vividly differentiates the role of $\mathrm{CNT}$ content in enhancing the elastic modulus and fracture toughness of the $\mathrm{A} 8 \mathrm{C}-\mathrm{SD}$ coating when compared to $\mathrm{A} 4 \mathrm{C}-\mathrm{SD}$ coating.

Since storage modulus falls in phase with the materials response, it is the direct measure in relating the resilience of a material. Stiffness associated with the content and distribution of CNTs is captured in the nanomechanical dynamic characterization. Corresponding fracture toughness of the plasma sprayed coatings directly correlate with the modulus maps. Local area evaluation of modulus in the coatings, therefore, is a direct measure of increase in fracture toughness. Nanomechanical analysis becomes an unmatched tool in mapping mechanical properties of the plasma sprayed nanocomposite coatings. Localized effects of dispersion strengthening by CNT content and CNT dispersion are clearly visualized through nanomechanical data. Modulus mapping, therefore, completes the picture of associated fracture toughening via CNT reinforcement. 

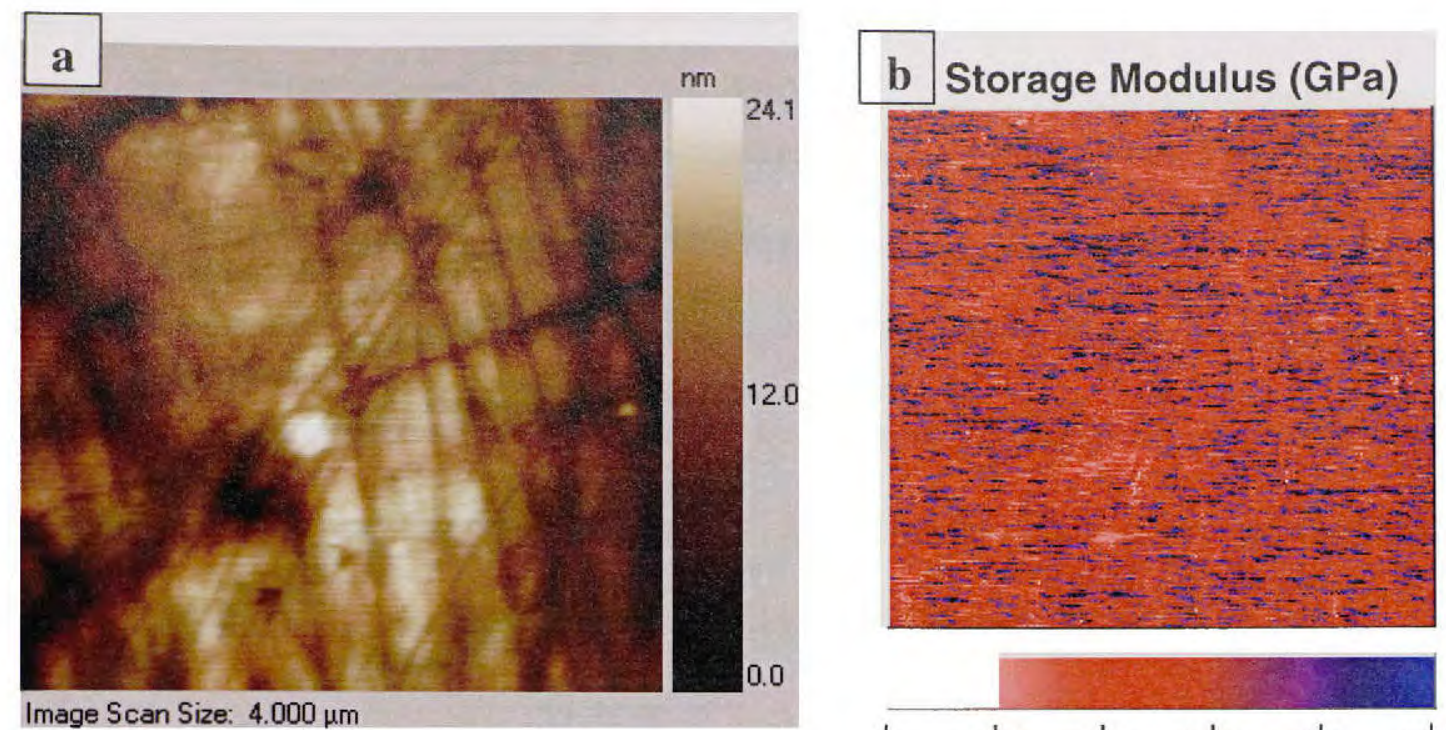

Image Scan Size: $4.000 \mu \mathrm{m}$
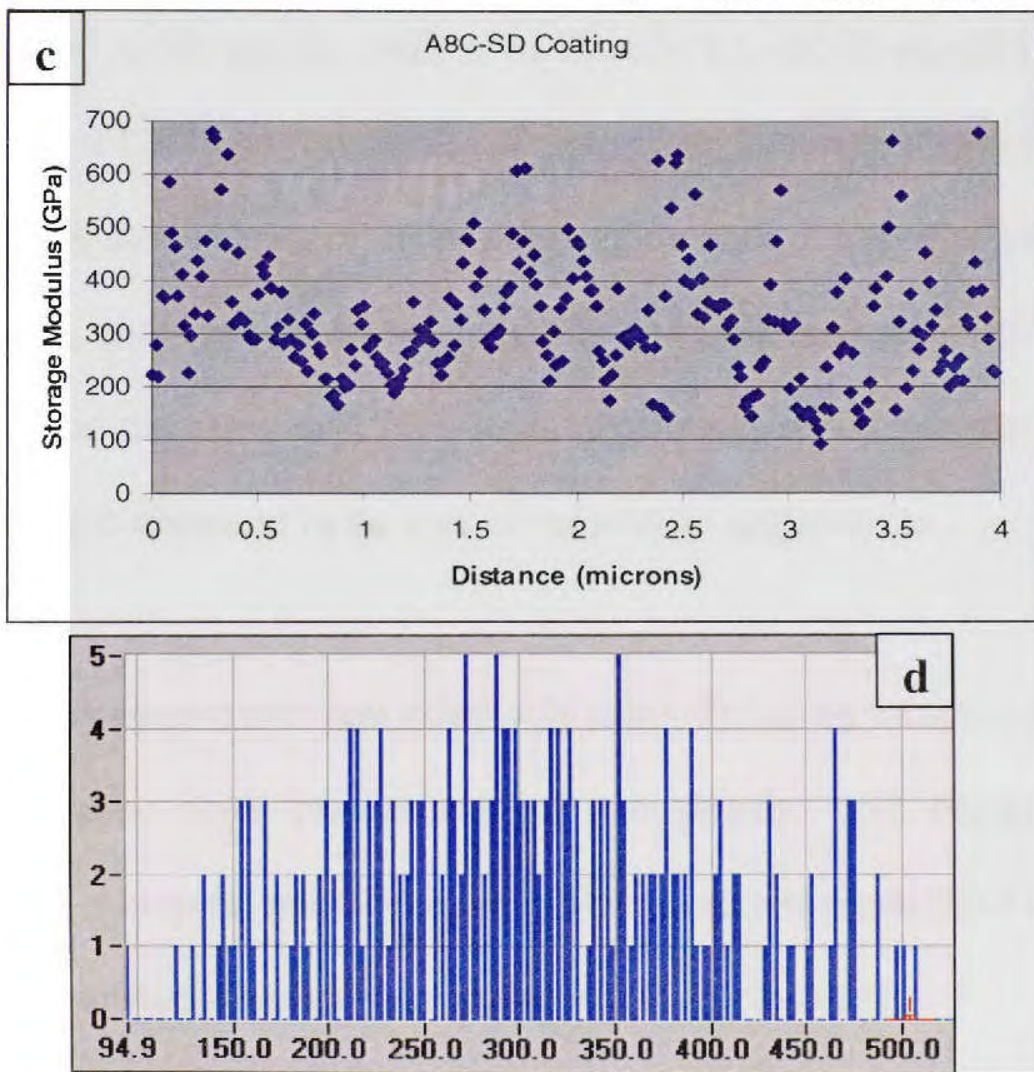

Storage Modulus

Fig. 4.71: Plasma sprayed A8C-SD coating showing, a)Surface topography,

b) Modulus mapping, c) Line scan distribution of storage modulus with distance, and d) Histogram of modulus distribution. 


\section{CONCLUSIONS}

CNT reinforced aluminum oxide nanocomposite coatings have been successfully synthesized using plasma spray technique. Fracture toughness enhancement of upto $57 \%$ have been attained in the engineered coatings. Role of CNT retention and dispersion is elucidated towards understanding various toughening mechanisms. A detailed list of conclusions regarding the powder treatment, microstructural and phase characterization, $\mathrm{Al}_{2} \mathrm{O}_{3} / \mathrm{CNT}$ interface, and mechanical properties are presented herewith.

1. Blending of A-SD powder with 4 wt. \% CNTs (A4C-B powder) has resulted dispersion of CNTs on the surface of powder agglomerate, and accumulation of CNTs in the dome cavity of the powder agglomerate. Composite spray drying of nano $\mathrm{Al}_{2} \mathrm{O}_{3}$ and 4 wt. $\% / 8$ wt. \% CNTs (A4C-SD and A8C-SD respectively) have resulted dispersion of CNTs uniformly throughout the powder agglomerate. CNTs are also dispersed in the core of the powder agglomerate.

2. Plasma spray parameters were effectively controlled using inflight particle sensor. In combination with pretreatment of the powder and controlled process parameters, a bimodal microstructure (fully melted and resolidified structure, and solid state sintered structure) was engineered in $\mathrm{Al}_{2} \mathrm{O}_{3}$ matrix. 
3. CNTs are successfully retained in the plasma sprayed A4C-B, A4C-SD, and A8CSD coatings as confirmed via microstructural characterization and Raman analysis. Retention of CNTs is attributed to the rapid kinetics (dwell time of $4 \times 10^{-4} \mathrm{~s}$ ) involved in the plasma spraying.

4. Distribution and content of CNTs in the powder agglomerate have strong effect on the thermal exposure observed by powder particles in the plasma plume (measured via inflight particle monitor). A4C-B coating experienced temperatures of $2898 \mathrm{~K}$ (with velocity of $271 \mathrm{~m} / \mathrm{s}$ ), which is higher than that of A-SD coating $(2512 \mathrm{~K}, 289 \mathrm{~m} / \mathrm{s})$. A4C-SD and A8C-SD coatings experienced reduced thermal exposure $(2332 \mathrm{~K}(244 \mathrm{~m} / \mathrm{s})$, and $2241 \mathrm{~K}(232 \mathrm{~m} / \mathrm{s})$ respectively) owing to coreCNTs serving as 'heat-sinks'.

5. Generation of metastable $\gamma-\mathrm{Al}_{2} \mathrm{O}_{3}$ phase is dependent on the thermal exposure experienced by CNTs available on the surface of powder agglomerate. Bimodal crystallite size $\left(\sim 20 \mathrm{~nm}\right.$ for $\gamma-\mathrm{Al}_{2} \mathrm{O}_{3}$ and $\geq 40 \mathrm{~nm}$ for $\left.\alpha-\mathrm{Al}_{2} \mathrm{O}_{3}\right)$ is obtained.

6. Addition of 4 wt. $\%$ CNT (in A4C-B coating) showed an improvement of $20 \%$ in fracture toughness (from 3.22 to $3.86 \mathrm{MPa} \mathrm{m}{ }^{1 / 2}$ ) when compared to AC-SD coating. But, addition of dispersed 4 wt.\% CNTs (A4C-SD) coating showed an improvement of $43 \%$ (to $4.60 \mathrm{MPa} \mathrm{m}^{1 / 2}$ ). This evinces enhancement of fracture toughness just because of $\mathrm{CNT}$ dispersion owing to powder treatment. Further, increasing the $\mathrm{CNT}$ content to 8 wt.\% (A8C-SD coating), fracture toughness 
increased to $5.04 \mathrm{MPa} \mathrm{m}^{1 / 2}$ which is indicative of fracture toughness enhancement (by $57 \%$ ) because of increase in CNT content.

7. Toughening features such as impact alignment of CNTs along interface, CNT hooks, CNT bridge formation, CNT fusion on $\mathrm{Al}_{2} \mathrm{O}_{3}$ surface, and CNT chain loop formation are observed. Enhancement of toughness is attributed to mechanisms such as interfacial shear strengthening, high bending stiffness, enhanced energy absorption, and good torsional stiffness associated with excellent mechanical properties of CNTs.

8. Improved wetting is observed between molten $\mathrm{Al}_{2} \mathrm{O}_{3}$ and $\mathrm{CNT}$ surface. Phenomenon like CNT bridging, CNT entrapment in the solid-state sintered region, and CNT mesh formation are dominated by interplay of surface tension and capillarity. As estimated from theoretical computation modeling, high surface tension $(\sim 236 \mathrm{nN})$ was pinned for promoting high meniscus height, whereas increased capillarity $(\sim 12.6 \mathrm{nN})$ was reasoned for CNT meshing.

9. Computational ab-initio modeling was performed to evince electronic charge density perturbations observed along the $\mathrm{Al}_{2} \mathrm{O}_{3}-\mathrm{CNT}$ interface. Overlapping of electron cloud at the $\mathrm{Al}_{2} \mathrm{O}_{3}$ - $\mathrm{CNT}$ interface in forming pseudo metallic bonding (charge density $>0.3$ ), which is indicative of adhesion and good wettability at the interface. Effect of rapid kinetics inherent to the plasma spraying is attributed to enhanced-wettability associated in the $\mathrm{Al}_{2} \mathrm{O}_{3}-\mathrm{CNT}$ system. 
10. Effect of CNT dispersion in enhancing the elastic modulus is identified using nanoindentation dynamic modulus mapping. A-SD $(\sim 210 \mathrm{GPa})$, A4C-B $(\sim 250$ $\mathrm{GPa})$, A4C-SD ( 280 GPa) and A8C-SD ( 325 GPa and $420 \mathrm{GPa})$, clearly indicate increase in the modulus with increasing CNT content and distribution. 


\section{RECOMMENDATIONS FOR FUTURE}

Every dissertation is a connecting link between new paradigm of science and engineering tying its free end with the advancement of scientific knowledge. Plasma spraying of CNT reinforced $\mathrm{Al}_{2} \mathrm{O}_{3}$ was performed to produce enhanced toughening of the nanocomposite coatings using pretreatment of powders. Detailed optimization, microstructural characterization, phase analysis and mechanical property evaluation of the coatings have elicited toughness improvement of upto $57 \%$ when compared to that of unreinforced coating. During the completion of the dissertation or a major research project, it is always the desire to hover on a few more dimensions of research. Perspectives from different directions and endless discussions on some of the undiscovered aspects germinate the seeds of further development and are recommended for future dwelling as follows:

\subsection{Optimization of CNT Content in Aluminum Oxide Matrix}

Fracture toughness enhancement of $43 \%\left(4.60 \mathrm{MPa} \mathrm{m}^{1 / 2}\right)$ was observed in $\mathrm{A} 4 \mathrm{C}$ SD coating when compared to that of A-SD coating. Agglomeration of CNT in A8C-SD coating could not be fully avoided even though it displayed fracture toughness enhancement of $57 \%\left(5.04 \mathrm{MPa} \mathrm{m}^{1 / 2}\right)$. This is indicative of intermediate composition of CNT (between 4 and 8 wt.\% CNTs) in $\mathrm{Al}_{2} \mathrm{O}_{3}$ matrix where CNTs can be full dispersed without agglomeration to provide highest toughness. A systematic parametric study could be performed to optimize CNT content and its uniform dispersion in providing maximum fracture toughness. 


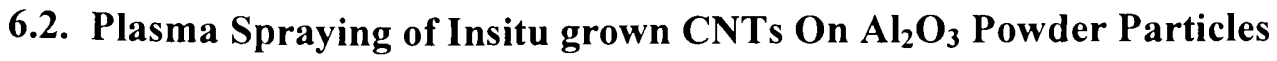

As observed in the current research that CNT dispersion is critical in imparting enhanced fracture toughness. Instead of adding CNTs separately in the powder feedstock via blending and spray drying, CNTs can be grown insitu on $\mathrm{Al}_{2} \mathrm{O}_{3}$ powders. Advantage of insitu grown CNTs can provide the required anchoring and dispersion throughout the matrix in each powder particle. However, a critical control is required on insitu grown CNT length and their density on each $\mathrm{Al}_{2} \mathrm{O}_{3}$ powder particle. CNT length should be short enough to avoid entanglement and subsequent clogging of powders during plasma spray. On the other hand, CNTs should be long enough to allow inter-splat anchoring.

\subsection{Molecular Dynamics Simulation of $\mathrm{Al}_{2} \mathrm{O}_{3} / \mathrm{CNT}$ Interface}

Energy of formation could be compared for different structures such as $\mathrm{Al}_{2} \mathrm{O}_{3}, \alpha$ $\mathrm{Al}_{2} \mathrm{O}_{3} / \mathrm{CNT}$ interface and $\gamma-\mathrm{Al}_{2} \mathrm{O}_{3} / \mathrm{CNT}$ interface in deriving stable atomic configuration. Molecular dynamics approach can be applied to calculate force of separating CNTs from $\mathrm{Al}_{2} \mathrm{O}_{3}$ surface. Measuring the force of separation points out the work of adhesion required that would be absorbed by interface before fracture. This can directly indicate the dependence of interface on the fracture toughness related to the modeled system.

\subsection{Hot-Isostatic Pressing of Plasma Sprayed Nanocomposites}

Role of secondary densification processing in enhancing the fracture toughness of the plasma sprayed nanocomposites can throw light on the role of porosity in imparting toughening behavior. Retention and distribution of CNTs in the hot-isostatically pressed (HIPped) coatings will elucidate the damage tolerance of CNTs under high temperature 
and pressure. Consequently, balance between the retention of CNTs (and its damage) and the porosity closure will decide the overall toughening of the nanocomposite. HIP study will differentiate the role of porosity versus $\mathrm{CNT}$ on the fracture toughness of $\mathrm{Al}_{2} \mathrm{O}_{3}$ CNT nanocomposite coating.

\subsection{Estimating Flexural Strength of Plasma Sprayed $\mathrm{Al}_{2} \mathrm{O}_{3}-\mathrm{CNT}$ Nanocomposite}

To estimate the flexural strength of the bulk structure, three-point bend test can be utilized on freestanding samples. But, one of the major challenges is to machine such a thick and large sample for 3-point test from these brittle materials. Flexural strength should be evaluated in plasma sprayed and hipped conditions to differentiate the role of porosity in toughening (and/or strengthening) the ceramic nanocomposite.

\subsection{Sliding Wear Evaluation of $\mathrm{Al}_{2} \mathrm{O}_{3}$ with $\mathrm{CNT}$ Addition}

One of the major applications of $\mathrm{Al}_{2} \mathrm{O}_{3}$ is as wear resistant coatings. Effect of CNT content in improving the wear resistance by anchoring the splats should be studied for its tribological performance. Hence sliding wear resistance of the novel coating with different $\mathrm{CNT}$ reinforcement should be evaluated. In addition, investigation of $\mathrm{Al}_{2} \mathrm{O}_{3}$ CNT frictional coefficient and abrasion resistance can explain its usage in thermal liners and insulating systems. 


\section{LIST OF REFERENCES}

[1] Balani K, Agarwal A, McKechnie T. Near net shape fabrication via vacuum plasma spray forming. Trans. Indian Inst. Met. 2006;59(2):237-44.

[2] Chen $\mathrm{CH}$, Awaji H. Temperature dependence of mechanical properties of aluminum titanate ceramics. Journal of the European Ceramic Society 2007;27:13-18.

[3] Karagedov GR, Lyakhov NZ. Preparation and sintering of nanozisesd alphaAl2O3 powder. Nanostructured Materials 1999;11:559-72.

[4] Katsuda Y, Gerstel P, Narayanan J, Bill J, Aldinger F. Reinforcement of precursor-derived Si-C-N ceramics with carbon nanotubes. ECERS 2005.

[5] Li T, Li Q, Fuh JYH, Yu PC, Lu L, Wu CC. Effects of AGG on fracture toughness of tungsten carbide. Materials Science and Engineering A 2007;445-446:58792.

[6] Lima RS, Marple BR. From APS to HVOF spraying of conventional and nanostructured titania feedstock powders: a study on the enhancement of the mechanical properties. Surface \& Coatings Technology 2006;200:3248-437.

[7] Ma Q-S, Chen Z-H, Zheng W-W, Hu H-F. Processing and characterization of particles reinforced $\mathrm{Si}-\mathrm{O}-\mathrm{C}$ composites via pyrolysis of poludiloxane with $\mathrm{SiC}$ or/and $\mathrm{Al}$ fillers. Ceramics International 2005;31:1045-51.

[8] Monteverde F. Ultra-high temperature HfB2-SiC ceramics consolidated by hotpressing and spark plasma sintering. Journal of Alloys and Compounds 2007;428:197205.

[9] Peigney A, Flahaut E, Laurent C, Chastel F, Rousset A. Aligned carbon nanotubes in ceramic-matrix nanocomposites prepared by high-temperature extrussion. Chemical Physics Letters 2002;352:20-25.

[10] Sciti D, Monteverde F, Guicciardi S, Pezzotti G, Bellosi A. Microstructure and mechanical properties of $\mathrm{ZrB} 2-\mathrm{MoSi} 2$ ceramic composite produced by different sintering techniques. Materials Science and Engineering A 2006;434:303-09.

[11] Sun J, Gao L, Jin X. Reinforcement of alumina matrix with multi-walled carbon nanotubes. Ceramics International 2005;31:893-96.

[12] Turunen E, Varis T, Gustafsson TE, Keskinen J, Falt T, Hannula S-P. Parameter optimization of HVOF sprayed nanostructured alumina and alumina-nickel composite coatings. Surface \& Coatings Technology 2006;200(4987-4994). 
[13] Yu L, Ma Y, Zhou C, Xu H. Damping capacity and dynamic mechanical characteristics of the plasma-sprayed coatings. Materials Science \& Engineering A 2005;A 408:42-46.

[14] Levin I, Brandon D. Metastable Alumina Polymorphs: Crystal Structures and Transition Sequences. Journal of the American Ceramic Society 1998;81(8):1995-2012.

[15] Chen CK. Statistical simulation of microcrack toughening in advanced ceramics. ECERS 2005;25:3293-99.

[16] Seigel RW, Chang SK, Ash BJ, Stone J, Ajayan PM, Doremus RW, et al. Mechanical behaviour of polymer and ceramic matriz nanocomposites. Scripta Materialia 2001;44:2061-64.

[17] Xia Z, Riester L, Curtin WA, Li H, Sheldon BW, Liang J, et al. Direct observation of toughening mechanisms in carbon nanotube ceramic matrix composites. Acta Materialia 2004;52:931-44.

[18] Xu L, Xie Z, Gao L, Wang X, Lian F, Liu T, et al. Synthesis, evaluation and characterization of alumina ceramics with elongated grains. Ceramics International 2005;31:953-58.

[19] Becher F, Hsueh CH, Angelini P, Tiegs TN. Theoretical and Experimental Analysis of the Toughening Behavior of Whisker Reinforcement in Ceramic Matrix Composites: Pergamon; 1989.

[20] Maensiri S, Laokul P, Klinkaewnarong L, Amornkitbamrung V. Carbon nanofiber reinforced alumina nanocomposites: Fabrication and mechanical properties. Materials Science and Engineering A 2007;447:44-50.

[21] Balazsi C, Konya Z, Weber F, Biro LP, Arato P. Preparation and characterization of carbon nanotube reinforced silicon nitride composites. Materials Science \& Engineering C 2003;23:1133-37.

[22] Hwang GL, Hwang KC. Carbon nanotbe reinforced ceramics. Journal of Materials Chemistry 2001;11:1722-25.

[23] Jitianu A, Cacciaguerra T, Benoit R, Delpeux S, Beguin F, Bonnamy S. Synthesis and characterization of carbon nanotubes- TiO2 nanocomposites. Carbon 2004;42:114751 .

[24] Kastritseas C, Smith PA, Yeomans JA. Thermal shock fracture in unidirectional fibre-reinforced ceramic-matrix composites. Composites Science and Technology 2005;65:1880-90. 
[25] Lupo F, Kamalakaran R, Scheu C, Grobert N, Ruhle M. Microstructural investigations on zirconium oxide-carbon nanotube composites synthesized by hydrothermal crystallization. Carbon 2004;42:1995-99.

[26] Ma RZ, Wu J, Wei BQ, Liang J, Wu DH. Processing and properties od carbon nanotubes-nano-SiC ceramic. Journal of Materials Science 1998.

[27] Ning J, Zhang J, Pan Y, Guo J. Fabrication and mechanical properties of SiO2 matrix composites reinforced by carbon nanotube. Materials Science \& Engineering A 2003;A357:392-96.

[28] Ning J, Zhang J, Pan Y, Guo J. Surfactants assisted processing of carbon nanotube-reinforced $\mathrm{SiO} 2$ matrix composites. Ceramics International 2004;30:63-67.

[29] Rul S, Lefevre-schlick F, Capria E, Laurent C, Peigney A. Percolation of singlewalled carbon nanotubes in ceramic matrix nanocomposites. Acta Materialia 2004;52:1061-67.

[30] Zhang F, Shen J, Sun J. Processing and properties of carbon nanoutbes-nan-WCCo composites. Materials Science \& Engineering A 2004;A 381:86-91.

[31] Peigney A, Laurent C, Flahaut E, Rousset A. Carbon nanotubes in novel ceramic matrix nanocomposites. Ceramics International 2000;26:677-83.

[32] Wang YG, Bronsveld PM, DeHosson JTM. Ordering of octahedral vacancies in transition aluminas. Journal American Ceramic Society 1998;81:1655-60.

[33] Wang Y, Jiang S, Wang M, Wang S, Xiao TD, Strutt PR. Abrasive wear characteristics of plasma sprayed nanostructured alumina/titania coatings. Wear 2000;237:176-85.

[34] Li WQ, Gao L. Processing, microstructure and mechanical properties of $25 \% \mathrm{vol}$ YAG-Al203 Nanocomposites. Nanostructured Materials 1999;11:1073-80.

[35] Nakao W, Ono M, Lee S-K, Takahashi K, Ando K. Critical crack-healing condition for $\mathrm{SiC}$ whisker reinforced alumina under stress. Journal of the European Ceramic Society 2004;25:3649-55.

[36] Bansal P, Padture NP, Vasiliev A. Improved inerfacial mechanical propeties of Al2O3-13 wt \% TiO2 plasma-sprayed coatings derived from nanocrystalline powders. Acta Materialia 2003;51:2959-70.

[37] An J-W, You D-H, Lim D-S. Tribological properites of hot-pressed alumina-CNT composites. Wear 2004;255:677-81. 
[38] Anglada NF, Kaepgen M, Skakalova V, Weglikowska UD, Roth S. Synthesis and characterization of carbon nanotube-conducting polymer thin films. Diamond and Related Materials 2004;13:256-60.

[39] Laha T, Balani K, Agarwal A, Pati S, Seal S. Synthesis of nanostructured spherical aluminum oxide powders by plasma engineering. Metallurgical and Materials Transactions A 2005;36:301-09.

[40] Fang JC, Xu WJ. Plasma spray forming. Journal of Materials Processing Technology 2002;129:288-93.

[41] Fauchais P, Vardelle A, Dussoubs B. Quo Vadis Thermal Spraying? Journal of Thermal Spray Technology 2001;10(1):44-66.

[42] Laha T, Agarwal A, McKechnie T. Comparative evaluation of plasma and high velocity oxy-fuel spray formed carbon nanotube reinforced Al-based composite. In: The Minerals Metals \& Materials Society; 2005.

[43] Laha T, Agarwal A, McKechnie T, Seal S. Synthesis and characterization of plasma spray formed carbon nanoutbe aluminum composite. Materials Science \& Engineering A 2004;A381:249-58.

[44] Laha T, Kuchibhatla S, Seal S, Li W, Agarwal A. Interfacial phenomena in thermally sprayed multiwalled carbon nanotube reinforced aluminum nanocomposite. Acta Materialia 2007;55:1059-66.

[45] Souza JVC, Santos C, Kelly CA, Silva OMM. Development of alpha-SiAlON$\mathrm{SiC}$ ceramic composites by liquid phase sintering. International Journal of Refractory Metals \& Hard Materials 2007;25:77-81.

[46] Zhang T, Zhang Z, Zhang J, Jiang D, Lin Q. Preparation of SiC ceramic by aqueous gelcasting and pressuless sintering. Materials Science and Engineering $A$ 2007;443:257-61.

[47] Kim YW, Chun YS, Nishimura T, Mitomo M, Lee YH. High temperature strength of silicon carbide ceramis sintered with rare-earth oxide and aluminum nitride. Acta Materialia 2007;55:727-36.

[48] Eblagon F, Ehrle B, Graule T, Kuebler J. Development of silicon nitride/silicon carbide composites for wood-cutting tools. Journal of European Ceramic Society 2007;27:419-28.

[49] Gu M, Huang C, Zou B, Liu B. Effect of (Ni, Mo) and TiN on the microstructure and mechanical properties of TiB2 ceramic tool materials. Materials Science and Engineering A 2006;433:39-44. 
[50] Balog M, Keckes J, Schoeberl T, Galusek D, Hofer F, Krest'an J, et al. Nano/macro-hardness and fracture resistance of Si3N4/ SiC composites with upto 13 wt.\% of SiC nano-particles. Journal of the European Ceramic Society 2007;27:2145-52.

[51] Hemker KJ. Understading How Nanocrystalline Metals Deform. Science 2004;304:221-22.

[52] Vassen JR. Densification and Grain Growth of Nano-Phase Ceramics. DKG 1999;76(4):19-22.

[53] Balani K, Gonzalez G, Agarwal A, Hickman R, O'Dell JS, Seal S. Synthesis, microstructural characterization, and mechanical property evaluation of vacuum plasma sprayed tantalum carbide. Journal of American Ceramics Society 2006;89(4):1419-25.

[54] Balani K, Anderson R, Laha T, Andara M, Tercero J, Crumpler E, et al. Plasmasprayed carbon nanotube reinforced hydroxyapatite coatings and their interaction with human osteoblasts in vitro. Biomaterials 2007;28:618-24.

[55] Upadhya K, Yang JM, Hoffman WP. Materials for ultrahigh temperature structural applications. American Ceramic Society Bulletin 1997:51-56.

[56] Kim YW, Chun YS, Nishimura T, Mitomo M, Lee YH. High temperature strength of silicon carbide ceramics sintered with rare-earth oxide and aluminum nitride. Acta Materialia 2007;55:727-36.

[57] Krnel K, Stadler Z, Kosmac T. Preparation and properties of C/C-SiC nanocomposites. Journal of European Ceramic Society 2007;27:1211-16.

[58] Xu Y, Zhang Y, Cheng L, Zhang L, Lou J, Zhang J. Preparation and friction behavior of carbon fiber reinforced silicon carbide matrix composites. Ceramics International 2007;33:439-45.

[59] Anstis GR, Chantikul P, Lawn BR, Marshall DB. A Critical Evaluation of Indentation Techniques for Measuring Fracture Toughness: I, Direct Crack Measurements. Journal of the American Ceramic Society 1981;64(9):533-38.

[60] Upadhya K, Yang JM, Hoffman WP. Materials for ultrahigh temperature structural applications. The American Ceramic Society Bulletin 1997;76(12):51-56.

[61] Miyayama M, Koumoto K, Yanagida H. Engineering properties of single oxides. Materials Park, OH: ASM International; 1991, p. 748-57.

[62] Wang HZ, Gao L, Gui LH, Guo JK. Preparation and Properties of Intragranular Al2O3-SiC Nanocomposites. Nanostructured Materials 1998;10(6):947-53. 
[63] Bargeron CB, Benson RC, Jette AN, Phillips TE. Oxidation of Hafnium Carbide in the temperature range 1400 to 2060 C. Journal of American Ceramics Society 1993;76(4):1040-46.

[64] Mattuck JBB. High temperature oxidation. Journal of Electrochemical Society 1967;114(10):1030-33.

[65] Barsoum MW, El-Raghy T. The MAX Phases: Unique New Carbide and Nitride Materials. American Scientist 2001;89:334-43.

[66] Miao X, Sun D, Hoo PW, Liu J, Hu Y, Chen Y. Effect of titania addition on yttria-stabilised tetragonal zirconia ceramics sintered at high temperatures. Ceramics International 2004;30:1041-47.

[67] Anne G, Put S, Vanmeensel K, Jiang D, Vleugels J, Biest OVd. Hard, tough and strong ZrO2-WC composites from nanosized powders. Journal of European Ceramic Society $2005 ; 25: 55-63$.

[68] Khor KA, Dong ZL, Gu YW. Plasma sprayed functionally graded thermal barrier coatings. Materials Letters 1999;38:437-44.

[69] Bhaduri S, Bhaduri SB. Microstructural and mechanical properties od nanocrystalline spinel and related composites. Ceramics International 2002;28(153-158).

[70] Swygenhoven HV, Weetman JR. Deformation in nanocrystalline metals. Materials Today 2006;9:24-31.

[71] Wu J, Li B, Guo J. The influece of addition of AlN particles on mechanical properties of $\mathrm{SiO} 2$ matrix composites doped with AlN particles. Materials Letters $1999 ; 41: 145-48$.

[72] Krishnan R, Dash S, Kesavamoorthy R, Rao CB, Tyagi AK, Raj B. Laser surface modification and characterization of air plasma sprayed alumina coatings. Surface \& Coatings Technology 2006;200(2791-2799).

[73] Gell M, Jordan EH, Sohn YH, Godberman D, Shaw L, Xiao TD. Development and implementation of plasma sprayed nanostructured ceramic coatings. Surface \& Coatings Technology 2001;146-147:48-54.

[74] Devi MU. New phase formation in Al2O3-based thermal spray coatings. Ceramics International 2004;30:555-65.

[75] Shaw LL, Goberman D, Ren R, Gell M, Jiang S, Wang Y, et al. The dependency of microstructure and properties of nanostructured coatings on plasma spray conditions. Surface and Coatings Technology 2000;130:1-8. 
[76] Jordan EH, Gell M, Sohn YH, Goberman D, Shaw L, Jiang S, et al. Fabrication and evaluation of plasma sprayed nanostructured alumina-titania coatings with superior properties. Materials Science and Engineering A 2001;301:80-89.

[77] Kabacoff LT. Nanoceramic coatings exhibit much higher toughness and wear resistance than conventional coatings. AMPTIAC Newsletter 2002:37-42.

[78] Chen H, Ding CX. Nanostructured zirconia coating prepared by atmospheric plasma spraying. Surface \& Coatings Technology 2002;150:31-36.

[79] Kulkarni A, Wang Z, Nakamura T, Sampath S, Goland A, Herman H, et al. Comprehensive microstructural characterization and predictive property modeling of plasma sprayed zirconia coatings. Acta Materialia 2003;51:2457-75.

[80] Gang J, Morniroli JP, Grosdidier T. Nanostructures in thermal spray coatings. Scripta Materialia 2003;48:1599-604.

[81] Zhu YC, Yukimura K, Ding CX, Zhang PY. Tribological properties of nanostructured and conventional WC-Co coatings deposited by plasma spraying. Thin Solid Films 2001;388:277-82.

[82] He J, Lee M, Schoenung JM, Shin DH, Lavernia EJ. Thermal stability of nanostructured $\mathrm{Cr} 3 \mathrm{C} 2-\mathrm{NiCr}$ coatings. Journal of Thermal Spray Technology 2001;10(2):293-300.

[83] Karthikeyan J, Berndt CC, Tikkanen J, Wang JY, King AH, Herman H. Nanomaterial powders and deposits prepared by flame spray processing of liquid precursors. Nanostructured Materials 1997;8(1):61-74.

[84] Jadhav A, Padture NP, Wu F, Jordan EH, Gell M. Thick ceramic thermal barrier coatings with high durability deposited using solution-precursor plasma spray. Materials Science \& Engineering A 2005;A 405:313-20.

[85] Hong SJ, Viswanathan V, Rea K, Patil S, Deshpande S, Georgieva P, et al. Plasma spray formed near-net-shape $\mathrm{MoSi} 2-\mathrm{Si} 3 \mathrm{~N} 4$ bulk nanocomposites-structure property evaluation. Materials Science and Engineering:A 2005;404:165-72.

[86] Eigen N, Gärtner F, Klassen T, Aust E, Bormann R, Kreye H. Microstructures and properties of nanostructured thermal sprayed coatings using high-energy milled cermet powders. Surface and Coatings Technology 2005; 195:344-57.

[87] Balani K, Gonzalez G, Agarwal A, Hickman R, O'Dell JS. Synthesis, Microstructural Characterization, and Mechanical Property Evaluation of Vacuum Plasma Sprayed Tantalum Carbide. Journal of American Ceramic Society 2006;89(4):1419-25. 
[88] Tului M, Marino G, Valente T. Plasma spray deposition of ultra high temperature ceramics. Composites Science and Technology 2006;201:2103-08.

[89] G. Xie, X. Lin, K. Wang, X. Mo, D. Zhang, P. Lin. Corrosion characteristics of plasma-sprayed $\mathrm{Ni}$-coated WC coatings comparison with different post-treatment. Corrosion Science 2007;49:662-671.

[90] Agarwal A, McKechnie T, Seal S. Net shape nanostructured aluminum oxide structures fabricated by plasma spray forming. Journal of Thermal Spray Technology 2003;12:350-59.

[91] Devi U. Microstructure of Al2O3-SiC nanocomposite ceramic coatings prepared by high velocity oxy-fuel flame spray process. Scripta Materialia 2004;50(7):1073-78.

[92] Goswami R, Herman H, Sampath S, Jiang X, Tian Y, Halada G. Plasma sprayed Mo-Mo oxide nanocomposites: synthesis and characterization. Surface and Coatings Technology 2001;141:220-26.

[93] Liang B, Liao H, Ding C, C. Coddet. Nanostructured zirconia-30 vol.\% alumina composite coatings deposited by atmospheric plasma spraying. Thin Solid Films 2005;484:225-31

[94] Goberman D, Sohn YH, Shaw L, Jordan E, Gell M. Microstructure development of $\mathrm{Al} 2 \mathrm{O} 3-13 \mathrm{wt} . \% \mathrm{Ti} 02$ plasma sprayed coatings derived from nanocrystalline powders. Acta Materialia 2002;50:1141-52.

[95] Lima RS, Marple BR. High Weibull modulus HVOF titania coatings. Journal of Thermal Spray Technology 2002;12:240-49.

[96] Lima RS, Marple BR. Optimized HVOF Titania Coatings. Journal of Thermal Spray Technology 2003;12:360-69.

[97] Zhu YC, Ding CX. Plasma spraying of porous nanostructured TiO2 film. Nanostructured Materials 1999;11(3):319-23.

[98] Lima RS, Kucuk A, Berndt CC. Integrity of nanostructured partially stabilized zirconia after plasma spray processing. Materials Science and Engineering A 2001;313:75-82.

[99] Li H, Khor KA, Kumar R, Cheang P. Characterization of hydroxyapatite/nanozirconia composite coatings deposited by high velocity oxy-fuel (HVOF) spray process. Surface \& Coatings Technology 2004;182:227-36.

[100] Gledhill HC, Turner IG, Doyle C. In vitro fatigue behaviour of vacuum plasma and detonation gun sprayed hydroxyapatite coatings. Biomaterials 2001;22(11):1233-40. 
[101] Herman H, Sampath S. Thermal Spray Coatings. 1n: Stern KH, editor. Metallurgical and Ceramic Protective Coatings. London: Chapman and Hall; 1996.

[102] Johnson WA, Kopatz NE, Yoder EB. Fine Powders Produced by Plasma Processing. In: Proceedings of Progress in Powder Metallurgy; 1986: Metal Powder Industries Federation; p. 775-89.

[103] Herman H, Sampath S. Metallurgical and Ceramic Protective Coatings. In: Stern KH, editor.: Chapman \& Hall, London; 1996. p. 261-89.

[104] Wang GX, Prasad V. Rapid Solidification: Fundamentals and Modeling. In: Tien CL, editor.; 2000. p. 207-305.

[105] Khor KA, Gu YW, Pan D, Cheang P. Microstructure and mechanical properties of plasma sprayed HA/YSZ/Ti-6Al-4V composte coating. Biomaterials 2004;25:4009-17.

[106] Skandan G. Processing of nanostructured Zirconia Ceramics. Nanostructured Materials 1995;5:111-26.

[107] Mohannadi Z, Moayyed AAZ, Mesgar ASM. Adhesive properties by indentation method of plasma-sprayed hydroxyapatite coatings. Applied Surface Science 2007;253:4960-65.

[108] Nomura N, Suzuki T, Yoshimi K, Hanada S. Microstructure and oxidation resistance of a plasma sprayed $\mathrm{Mo}-\mathrm{Si}-\mathrm{B}$ multiphase alloy casting. Intermetallics $2003 ; 11: 735-42$.

[109] Grosdidier T, Ji G, Bozzolo N. Hardness, thermal stability and yttrium distribution in nanostructured deposits obtained by thermal spraying from milled-Y2O3 reinforced- or atomized $\mathrm{FeAl}$ powders. Intermetallics 2006;14:715-21.

[110] Vasiliev AL, Padture NP, Ma X. Coatings of metastable ceramics deposited by solution precursor plasma spray: I. binary $\mathrm{ZrO} 2-\mathrm{Al} 2 \mathrm{O} 3$ system. Acta Materialia 2006;54:4913-20.

[111] Cao XQ. Spray-drying of ceramics for plasma-spray coatings. Journal of European Ceramic Society 2000;20:2433-39.

[112] Youshaw RA, Halloran JW. Compaction of spray-dried powders. Ceramic Bulletin 1982;61(2):227-30.

[113] Yamashita O, Kishimoto Y. Magnetic properties of Nd-Fe-B magnets sintered using granulated powder by spray-drying method. Powder Metallurgy 1998;41(3):17784. 
[114] Shaw LL, Goberman D, Ren R, Gell M, Jiang S, Wang Y, et al. The dependency of microstructure and properties of microstructured coatings on plasms spray conditions. Surface \& Coatings Technology 2000;130:1-8.

[115] Gell M. The potential for nanostructured materials in gas turbine engines. Nanostructured Materials 1995;6:997-1000.

[116] Ahmed I, Bergman TL. Three-Dimensional Simulation of Thermal Plasma Spraying of Partially Molten Ceramic Agglomerates. Journal of Thermal Spray Technology 2000;9(2):215-24.

[117] Li JF, Liao H, Wang XY, Coddet C, Chen H, Ding- CX. Plasma spraying of nanostructured partially yttria stabilized zirconia powders. Thin Solid Films 2004;460:101-15.

[118] Laha T, Balani K, Potens B, Andara M, Agarwal A, Patil S, et al. Plasma Engineered Nanostructured Spherical Ceramic Powders. In: TMS; 2004; p. 103-12.

[119] Piot LT, Berardo M, Charai A, Gastaldi J, Giorgi S. Microstructure of PlasmaSprayed TaC Coatings. Thin Solid Films 1994;248:12-17.

[120] Sampath S, Herman H. Rapid solidification and microstructure development during plasma spray deposition. Journal of Thermal Spray Technology 1996;5(4):445-56.

[121] Li J, Ye Y. Densification and grain growth of $\mathrm{Al} 2 \mathrm{O} 3$ nanoceramics during pressureless sintering. Journal of American Ceramic Society 2006;89(1):139-43.

[122] Gallas MR, Piermarini GJ. Bulk modulus and Young's modulus of nanocrystalline -alumina. Journal American Ceramic Society 1994;77:2917-20.

[123] Zbigniewlodziana, Topsoe NY, Norskov JK. A negative surface energy for alumina. Nature Materials 2004;3:289-93.

[124] Khanna A, Bhat DG. Nanocrystalline gamma alumina coatings by inverted cylindrical magnetron sputtering. Surface and Coatings Technology 2006;201:168-73.

[125] Zhan G-D, Kuntz J, Wan J, Garay J, Mukherjee AK. A novel processing route to develop a dense nanocrystalline alumina matric $(<100 \mathrm{~nm})$ nanocomposite material. Journal American Ceramic Society 2002;86:200-02.

[126] Chang S, Doremus RH, Schadler LS, Siegel RW. Hot-Pressing of nano-size alumina powder and the resulting mechanical properties. International Journal of Applied Ceramic Technology 2004;2:172-79.

[127] Wang Y, Fujimoto T, Maruyama H, Koga K. Precipitation of magnesium aluminum spinel from alumina-matrix solid solution: I, fundamental concept and precipitation behavior. Journal American Ceramic Society 2000;83:933-36. 
[128] Vasiliev AL, Poyato R, Padture NP. Single-wall carbon nanotubes at ceramic grain boundaries. Scripta Materialia 2007;56:461-63.

[129] Bahduri S, Bhaduri SB. Microstructural and mechanical properties of nanocrystalline spinel and related composites. Ceramics International 2002;28:153-58.

[130] Galusek D, Brydson R, Twigg PC, Riley FL. Wet erosive wear of alumina densified with magnesium silicate additions. Journal American Ceramic Society $2001 ; 84(1767-1776)$.

[131] Tuan WH, Chen RZ, Wang TC, Cheng CH, Kuo PS. Mechanical properties of $\mathrm{Al} 2 \mathrm{O} 3 / \mathrm{ZrO} 2$ composites. ECERS 2002;22:2827-33.

[132] Kumar AS, Durai AR, SOrnakumar T. Development of alumina-ceria ceramic composite cutting tool. International Journal of Refractory Metals \& Hard Materials 2004;22:17-20.

[133] Mekky W, Nicholson PS. R-curve modeling for Ni/Al2O3 laminates. Composites: B 2007;38:35-43.

[134] Miserez A, Müller R, Rossoll A, Weber L, Mortensen A. Particle reinforced metals of high ceramic content. Materials Science and Engineering A 2004;387-389:82231 .

[135] Parul Agrawal, C.T. Sun. Fracture in metal-ceramic composites. Composites Science and Technology 2004;64:1167-78.

[136] Kim IS. Thermal shock resistance of the al2o3-metal composites made by reactive infiltration of al into oxide fiber board. Materials Research Bulletin 1998;33(7):1069-75.

[137] Suryanarayana C, Koch CC. Nanostructured Materials. In: Suryanarayana C, editor. Non-Equilibrium Processing of Materials. Oxford, UK: Pergamon; 1999. p. $313-$ 72.

[138] Huang JY, Chen S, Wang ZQ, Kempa K, Wang YM, Jo SH, et al. Superplastic carbon nanotubes. Nature 2006:281.

[139] Poncharal P, Wang ZL, Ugarte D, Heer WA. Electrostatic deflections and electromechanical resonances of carbon nanotubes. Science 1999;283:1513-16.

[140] Lau K-T, Hui D. The revolutionary creation of new advanced materials-carbon nanotube composites. Composites B 2002;33(263-277).

[141] Andrews R, Weisenberger MC. Carbon nanotube polymer composites. Solid State \& Materials Science 2004;8:31-37. 
[142] Ci L, Bai J. The reinforcement role of carbon nanotubes in epoxy composites with different matrix stiffness. Composites Science and Technology 2006;66:599-603.

[143] Kearns JC, Shambaugh RL. Polypropylene fiber reinforced with carbon nanotubes. Journal of Applied Polymer Science 2002;86:2079-84.

[144] Cooper CA, Ravich D, Lips D, Mayer J, Wagner HD. Distribution and alignment of carbon nanotubes and nanofibrils in a polymer matrix. Composites Science and Technology 2002;62:1105-12.

[145] Li X, Gao H, Scrivens WA, Fei D, Xu X, Sutton MA, et al. Nanomechanical characterization of single-walled carbon nanotube reinforced epoxy composites. Nanotechnology 2004;15:1416-23.

[146] Qian D, Dickey EC, Andrews R, Rantell T. Load transfer and deformation mechanisms in carbon nanotube-polystyrene composites. Appl. Phys. Lett 2000;76(20):2868-70.

[147] Lourie O, Wagner HD. Evidence of dtress transfer and formation of fracture clusters in carbon nanotube-based composites. Composites Science and Technology 1999;59:975-77.

[148] Coleman JN, Cadek M, Blake R, Nicolosi V, Ryan KP, Belton C, et al. Highperformance nanotube-reinforced plastics: understanding the mechanism of strength increase. Advanced Functional Materials 2004;14(8):791-98.

[149] Song YS, Youn JR. Influence of dispersion states of carbon nanotubes on physical properties of expoxy nanocomposites. Carbon 2005;43:1378-85.

[150] Hernadi K, Ljubovic E, Seo JW, Forro L. Synthesis of MWNT-based composite materials with inorganic coating. Acta Materialia 2003;51(1447-1452).

[151] Chen L, Ye H, Gogotsi Y. Synthesis of boron nitride coating on carbon nanotubes. Journal American Ceramic Society 2004;87:147-51.

[152] Francke M, Hermann H, Wenzel R, Seifert G, Wetzig K. Modification of carbon nanostructures by high energy ball-milling under argon and hydrogen atmosphere. Carbon 2005;43:1204-12.

[153] Li GY, Wang PM, Zhao X. Mechanical behavior and microstructure of cement composites incorporating surface-treated multi-walled carbon nanotubes. Carbon 2005;43(1239-1245).

[154] Sun J, Gao L. Development of a dispersion process for carbon nanotubes in ceramic matrix by heterocoagulation. Carbon 2003;41:1063-68. 
[155] Fan JP, Zhuang DM, Zhao DQ, Zhang G, Wu MS, Wei F, et al. Toughening and reinforcing alumina matrix composite with single wall carbon nanotubes. Applied Physics Letters 2006;89:121910.

[156] Quang P, Jeong YG, Yoon SC, Hong SH, Kim HS. Consolidation of 1 vol.\% carbon nanotube reinforced metal matrix nanocomposite via equal channel angular pressing. Journal of Materials Processing Technology 2007:doi: 10.1016/j.jmatprotec.2006.11.116.

[157] Nai SML, Wei J, Gupta M. Improving the performance of lead-free solder reinforced with multi-walled carbon nanotubes. Materials Science and Engineering A 2006;423:166-69.

[158] Kuzumaki T, Ujiie O, Ichinose H, Ito K. Mechanical characteristics and preparation of carbon nanotube fiber-reinforced Ti composite. Advanced Engineering Materials 2002;2(7):416-18.

[159] Kim KT, Cha SI, Hong SH. Hardness and wear resistance of carbon nanotube reinforced $\mathrm{Cu}$ matrix nanocomposites. Materials Science and Engineering A 2007;449451:46-50.

[160] Dong SR, Tu JP, Zhang XB. An investigation of the sliding wear behavior of Cumatrix composite reinforced by carnon nanotubes. Materials Science \& Engineering A 2001;313:83-87.

[161] Morelli EC, Yang J, Couteau CE, Hernadi K, Seo JW, Bonjour C, et al. Carbon nanotube/magnesium composites. Phsica Status Solidi A 2004;201(8):R53-R55.

[162] Feng Y, Yuan HL, Zhang M. Fabrication and properties of silver-matrix composites reinforced by carbon nanotubes. Materials Characterization 2005;55:211-18.

[163] Laha T. Carbon nanotube reinforced aluminum based nanocomposite fabricated by thermal spray forming. Miami: Florida International University; 2006.

[164] Esawi A, Morsi K. Dispersion of carbon nanotubes (CNTs) in aluminum powder. Composites: Part A 2007;38:646-50.

[165] Curtin WA, Sheldon BW. CNT-reinforced ceramics and metals. Materials Today 2004:44-49.

[166] Zhan G-D, Kuntz JD, Wan J, Mukherjee AK. Single-wall carbon nanotubes as attractive toughening agents in alumina based nanocomposites. Nature Materials $2003 ; 2: 38-42$.

[167] Lim D-S, You D-H, Choi H-J, Lim S-H, Jang H. Effect of CNT distribution on tribological behavior of alumina-CNT composites. Wear 2005;259:539-44. 
[168] Fan J, Zhao D, Wu M, Xu Z, Song J. Preparation and microstructure of multi-wall carbon nanotubes toughened Al2O3 composite. Journal American Ceramic Society 2006;89:750-53.

[169] Balazsi CS, Weber F, Kover Z, Shen Z, Konya Z, Kasztovszky Z, et al. Application of carbon nanotubes to silicon nitride matrix reinforcements. Current Applied Physics 2006;6:124-30.

[170] Ye F, Liu L, Wang Y, Zhou Y, Peng B, Meng Q. Preparation and mechanical properties of carbon nanotube reinforced barium aluminosilicate glass-ceramic composites. Scripta Materialia 2006;55:911-14.

[171] Zhan G-D, Kuntz J, Wang J, Garay J, Mukherjee AK. Alumina-based nanocomposited consolidated by spark plasma sintering. Scripta Materialia 2002;47:73741.

[172] Wang X, Padture NP, Tanaka H. Contact-Damage-Resistant Ceramic/Single-Wall Carbon Nanotubes and Ceramic/Graphite Composites. Nature Materials 2004;3:539-44.

[173] Balazsi C, Shen Z, Konya Z, Kasztovszky Z, We'ber F, Ve'rtesy Z, et al. Processing of carbon nanotube reinforced silicon nitride composites by spark plasma sintering. Composites Science and Technology 2005;65:727-33.

[174] Balazsi C, Fe'nyi B, Hegman N, Ko"ve'r Z, F.We'ber, Ve'rtesy Z, et al. Development of CNT/Si3N4 composites with improved mechanical and electrical properties. Composites: Part B 2006;37:418-24.

[175] Mi W, Lin JYS, Li Y, Zhang B. Synthesis of vertically aligned carbon nanotube films on macroporous alumina substrates. Microporous and Mesoporous Materials 2005;81:185-89.

[176] Mo CB, Cha SI, Kim KT, Lee KH, Hong SH. Fabrication of carbon nanotube reinforced alumina matrix nanocomposite by sol-gel process. Materials Science and Engineering A 2005;A395:124-28.

[177] Cha SI, Kim KT, Lee KH, Mo CB, Hong SH. Strengthening and toughening of carbon nanotube reinforced alumina nanocomposite fabricated by molecular level mixing process. Scripta Materialia 2005;53:793-97.

[178] Bai JB, Vignes J-L, Fournier T, Michel D. A novel method for preparing preforms of porous Alumina and carbon nanotubes by CVD. Advanced Engineering Materials 2002;4:701-03.

[179] Kamalkaran R, Lupo F, Grobert N, Lozano-Castello D, Jin-Phillipp NY, Ruhle $M$. In-situ formation of carbon nanoutbes in an alumina-nanotube composite by spray pyrolysis. Carbon 2003;41:2737-41. 
[180] Wen S, Mho S, Yeo JH. Improved electrochemical capacitive characteristics of carbon nanotubes grown on the alumina templates with high pore density. Journal of Power Sources 2006;163:304-08.

[181] Qian D, Dickey EC, Andrews R, Rantell T. Load transfer and deformation mechanisms in carbon nanotube-polystyrene composites. Applied Physics Letter 2000;76:2868-70.

[182] Ramamurty U. Assesment of load transfer characteristics of a fiber-reinforced titanium-matrix composite. Composites Science and Technology 2005;65:1815-25.

[183] Zhang YC, Wang X. Thermal effects on interfacial stress transfer characteristics of carbon nanotubes/polymer composites. International Journal of Solids and Structures 2005;42:5399-412.

[184] Lim. D-S, You D-H, Choi H-J, Lim S-H, Jang H. Effect of CNT distribution on tribological behavior of alumina-CNT composites. Wear 2005;259:539-44.

[185] Zhan G-D, Mukherjee AK. Carbon nanotube reinfroced Alumina-based ceramics with novel mechanical, electrical and thermal properties. Int. J. Applied Ceramic Technology 2004;1(161-171).

[186] Jiang D, Thomson K, Kuntz JD, Ager JW, Mukherjee AK. Effect of sintering temperature on a single-wall carbon nanotube-toughened alumina-based nanocomposite. Scripta Materialia 2007;56(11):959-62.

[187] Duan R-G, Zhan G-D, Kuntz JD, Kear BH, Mukherjee AK. Processing and microstructure of high-pressure consolidated ceramic nanocomposites. Scripta Materialia 2004;51:1135-39.

[188] Duan R-G, Zhan G-D, Kuntz JD, Kear BH, Mukherjee AK. Spark plamsa sintering (SPS) consolidated cermaic composites from plasma-sprayed metastable Al2TiO5 powder nano-Al2O3, tiO2, and $\mathrm{MgO}$ powders. Materials Science \& Engineering A 2004;A373:180-86.

[189] Wood A. Using carbon nanotubes to reinforce ceramics. Chemical Week 2003:32.

[190] Zhan G-D, Kuntz JD, Garay JE, Mukherjee AK. Electrical properties of nanoceramics reinforced with ropes of single-walled carbon nanotubes. Applied Physics Letter 2003;83:1228-30.

[191] Zhan G-D, Kuntz JD, Wang H, Wang C-M, Mukherjee AK. Anisotropic thermal properties of single-wall-carbon-nanotube-reinforced nanoceramics. Philosopical Magazine Letters 2004;84:419-23. 
[192] Fernandez EM, Balbas LC, Borstel G, Soler JM. First principles calculation of the geometric and electronic structure of $(\mathrm{Al} 2 \mathrm{O} 3) \mathrm{n}(\mathrm{Ox})$ clusters with $\mathrm{n}<15$ and $\mathrm{x}=0,1,2$. Thin Solid Films 2003;428:206-10.

[193] Landry K, Kalogeropoulou S, Eustathopoulos N. Wettability of carbon by aluminum and aluminum alloys. Materials Science and Engineering A 1998;254:99-111.

[194] Ooi N, Adams JB. Electron density functional study of the aluminum-graphite interface. Journal of Computational Electronics 2004;3:51-56.

[195] Ownby PD, Li KWK, D. A. Weirauch J. High temperature wetting of sapphire by aluminum. Journal of American Ceramic Society 1991;74(6):1275-81.

[196] Siegel DJ, L. G. Hector J, Adams JB. Ab initio study of Al-ceramic interfacial adhesion. Physical Review B 2003;67:092105-1-4.

[197] Pakarinen OH, Foster AS, Paajanen M, Kalinainen T, Katainen J, Makkonen I, et al. Towards an accurate description of the capillary force in nanoparticle-surface interactions. Modeling and Simulation in Materials Science and Engineering 2005;13:1175-86.

[198] Fauchis P, Vardelle A, Dussoubs B. Journal of Thermal Spray Technology 2001;10:44-66.

[199] Fauchais P, Vardelle M, Vardelle A, Bianchi L. Ceramics International 1996;22(4):295-303.

[200] Meyer PJ, Hawley D. Thermal Spray Coatings: Properties, Processes and Application. Pittsburgh, PA; 1995, p. 57-63.

[201] Fauchais P, Vardelle A. International Journal of Thermal Sciences 2000;39:85270.

[202] Herman H, Sampath S. Metallurgical and Protective Coatings. In: Stern KH, editor.; 1996. p. 261-89.

[203] J.J.Kruzic, R.O.Ritchie. Determining the Toughness of Ceramics from Vickers Indentations Using the Crack-Opening Displacements: An Experimental Study. J. Am. Cer. Soc. V 2003;86(8):1433-36.

[204] Oliver WC, Pharr GM. An improved technique for determining hardness and elastic modulus using load and displacement sensing indentation experiments. J. Mater. Res. 1992;7(6):1564-83.

[205] Correa AA, Bonev SA, Galli G. Carbon under extreme conditions: Phase boundaries and electronic properties from first principles theory. Proceedings of the 
National Academy of Sciences of the United States of America 2006:doi: 10.1073/pnas.0510489103.

[206] Thostenson ET, Ren Z, Chou TW. Advances in the science and technology of carbon naotubes and their composites: a review. Comp. Sci. Tech. 2001;61:1899-912.

[207] Fan J, Zhao D, Wu M, Xu Z, Song J. J. Am. Ceram. Soc. 2006;89(2):750-3.

[208] Cha SI, Kim KT, Lee KH, Mo CB, Hong SH. Strengthening and toughening of carbon nanotube reinforced alumina nanocomposite fabricated by molecular level mixing. Scripta Mater. 2005;53:793-97.

[209] Balani K, Bakshi SR, Chen Y, Laha T, Agarwal A. Role of Powder Treatment and Carbon Nanotube Dispersion in the Fracture Toughening of Plasma-Sprayed Aluminum Oxide - Carbon Nanotube Nanocomposite. Journal of Nanoscience and Nanotechnology 2007.

[210] Li CJ, Yang GJ, Ohmori A. Relationship between particle erosion and lamellar microstructure for plasma-sprayed alumina coatings. Wear 2006;206:1 166-72.

[211] Song EP, Ahn J, Lee S, Kim NJ. Microstructure and wear resistance of nanostructured Al2O3-8wt.\%TiO2 coatings plasma-sprayed with nanopowders. Surface \& Coatings Technology 2006;201:1309-15.

[212] Chen Y, Gan CH, Zhang TN, Yu G, Bai PC, Kaplon A. Laser-surface-alloyed carbon nanotubes reinforced hydroxyapatite composite coating. Appl. Phys. Lett 2005;85:251905.

[213] Srivastava D, Wei C, Cho K. Nanomechanics of carbon nanotubes and composites. Appl. Mech. Rev 2003;56:215-30.

[214] Wen H-L, Chen Y-Y, Yen F-S, Huang C-Y. Size characterization of theta-and alpha-A12O3 crystallites during phase transformation. Nanostructured Materials 1998;11:89-101.

[215] Antis GR, Chantikul P, Lawn BR, Marshall DB. A Critical Evaluation of Indentation Techniques for Measuring Fracture Toughness: I, Direct Crack Measurements. Journal of the American Ceramic Society 1981;64(9):533-38.

[216] C.Q.Ru. Effective bending stiffness of carbon nanotubes. Physical Review B 2000;62(15):9973-76.

[217] C.H.Hsueh. Analytical evaluation of interfacial shear strength for fiber reinforced ceramic composites. J. Am. Ceram. Soc. 1988;71(6):490-93.

[218] Landry K, Kalogeropoulou S, Eustathopoulos N. Wettability of carbon by aluminum and aluminum alloys. Mater. Sci. Engg. A 1998;254(1-2):99-111. 
[219] Tian X, Bhushan B. The Micro-meniscus effect of a thin liquid film on the static friction of rough surface contact. Journal of Physics D: Applied Physics 1996;29:167-78.

[220] Balani K, Gonzalez G, Agarwal A, Hickman R, O'Dell JS, Seal S. Synthesis, Microstructural Characterization and Mechanical Property Evaluation of Vacuum Plasma Sprayed Tantalum Carbide. J. Am. Ceram. Soc. 2006;86(4):1419-25.

[221] Zhan GD, Kuntz JD, Duan RG, Mukherjee AK. Spark-Plasma Sintering of Silicon Carbide Whiskers ( $\mathrm{SiCw}$ ) Reinforced Nanocrystalline Alumina. J. Am. Ceram. Soc. 2004;87(12):2297-300.

[222] J.P.Salvetat, G.A.D.Briggs, J.M.Bonard, R.R.Bacsa, A.J.Kulik. Elastic and Shear Moduli of Single_Walled Carbon Nanotube Ropes. Physical Review Letters 1999;82(5):944-47.

[223] Srivastava D, C.Wei, K.Cho. Nanomechanics of carbon nanotubes and composites. Appl. Mech. Rev. 2003;56(2):215-30.

[224] Ishihara KN. Thermodynamics and Kinetics of Metastable Phase Formation. In: Suryanarayana C, editor. Non-Equilibrium Processing of Materials. Netherlands: Elsevier; 1999. p. 5-20.

[225] Porter DA, Easterling KE. Phase Transformations in Metals and Alloys. Second ed: CRC Press; 2001, p. 185-207.

[226] FactSage 5.1.3 Thermochemistry Software. Inc., ESM Software, Hamilton, OH.

[227] Meyer R, Ge Q, Lockemeyer J, Yeates Y, Lemanski M, Reinalda D, et al. An ab initio analysis of absorption and diffusion of silver atoms on alumina surfaces. Surface Science 2007;601(1):134-45. 


\begin{abstract}
APPENDICES
Appendix 1: As-Received CNT Characterization

SEM image of CNTs show an average diameter ranging between 40-70 nm, Fig. Al. Though CNTs show XRD diffraction peak of 2-theta around 26 degrees, Fig. A2, high intensity peaks arising from $\mathrm{Al}_{2} \mathrm{O}_{3}$ suppress $\mathrm{CNT}$ peaks in plasma sprayed nanocomposite coatings.
\end{abstract}

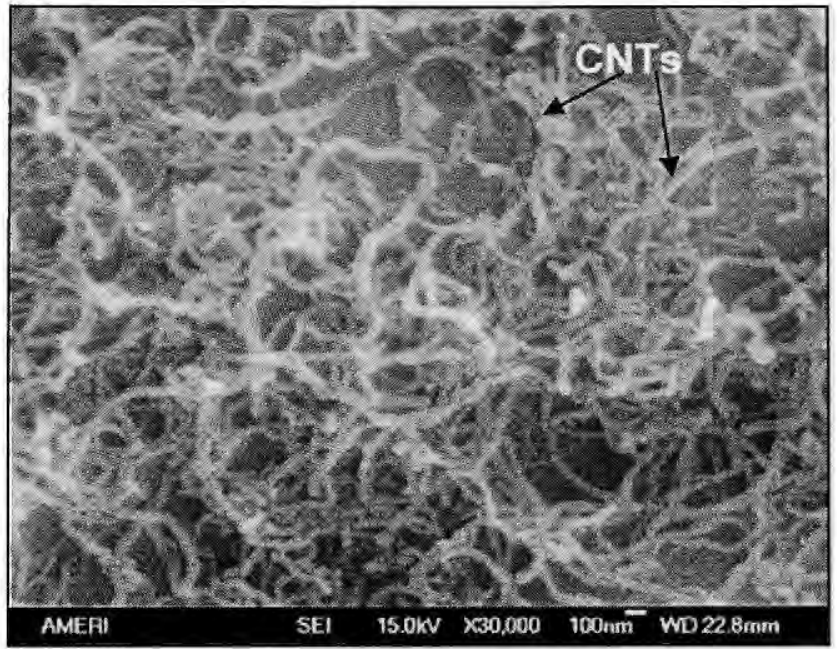

Fig. A1: SEM image showing CNT diameter in range of 40-70 nm.

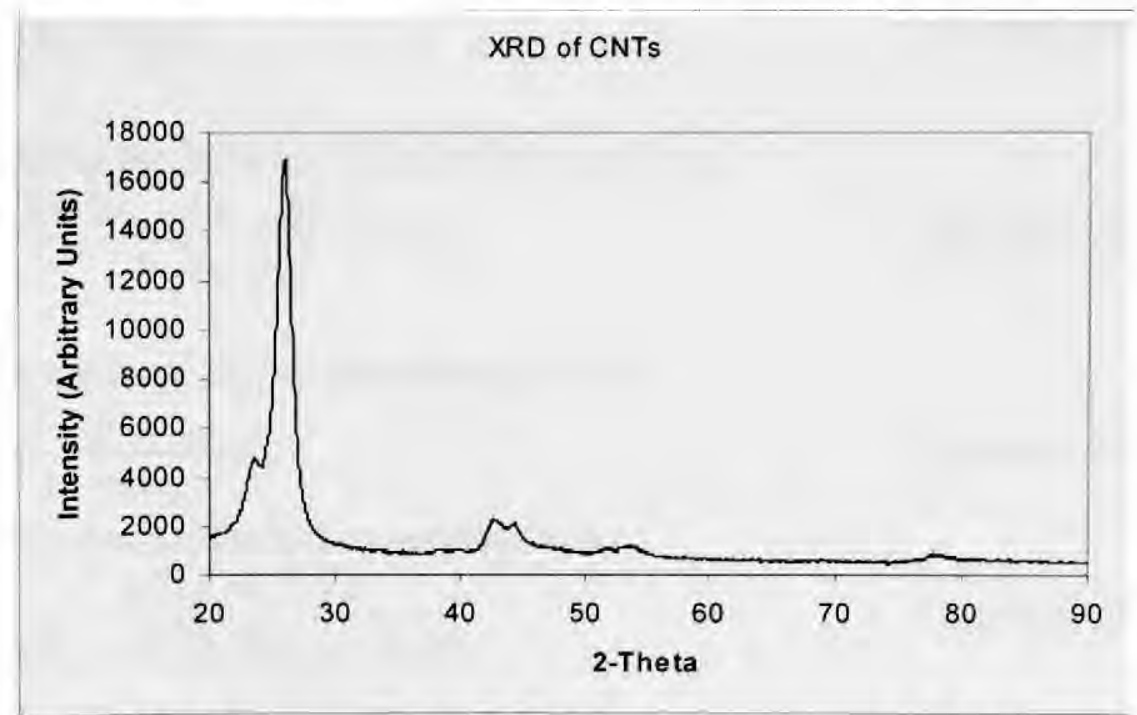

Fig. A2: XRD spectrum of CNTs showing intense peak at 26 degrees. 
Appendix 2: Modulus Enhancement with CNT Addition (Micromechanics Approach)

Micromechanics illuminate the interaction of matrix and reinforcement to determine the overall properties of composite depending on the individual properties constituents [41]. Hence the intention of adding CNT reinforcement in the $\mathrm{Al}_{2} \mathrm{O}_{3}$ matrix can be insinuated from micromechanics approach. Anisotropy, aspect ratio and morphology of the reinforcement are also accounted in mechanics model to describe the resulting properties of resulting combination. When overall response of the composite can be predicted via constituent relationships, it becomes easier to design the homogeneous (and generally isotropic) material [42].

Tensile and shear modulus of randomly distributed CNTs in a matrix can be described by eq. $\mathrm{A} 1$ and $\mathrm{A} 2$ as [43]:

$$
\begin{aligned}
& E=\frac{3}{8} E_{11}+\frac{5}{8} E_{22} \\
& G=\frac{1}{8} E_{11}+\frac{1}{4} E_{22}
\end{aligned}
$$

where longitudinal modulus $\left(E_{l I}\right)$ is given by eq. $\mathrm{A} 3$ as:

$$
E_{11}=\frac{1+2\left(l_{C N T} / d_{C N T}\right) \eta_{L} v_{C N T}}{1-\eta_{L} v_{C N T}} E_{m}
$$

Equation A3

and transverse modulus $\left(E_{22}\right)$ is given by eq. $\mathrm{A} 4$ as:

$$
E_{22}=\frac{1+2 \eta_{T} v_{C N T}}{1-\eta_{T} v_{C N T}} E_{m}
$$

and the other parameters are expressed as:

$$
\begin{aligned}
& \eta_{L}=\frac{\left(E_{C N N T} / E_{m}\right)-1}{\left(E_{C N T} / E_{m}\right)+2\left(l_{C N T} / d_{C N T}\right)} \\
& \eta_{T}=\frac{\left(E_{C N N T} / E_{m}\right)-1}{\left(E_{C N T} / E_{m}\right)+2}
\end{aligned}
$$

Equation A6 
Properties of such a composite are same in all the directions. By substituting the tensile moduli of $\mathrm{Al}_{2} \mathrm{O}_{3}$ (taken as $390 \mathrm{GPa}$, and Poisson's ratio of 0.22 ), one can calculate the overall effect of volume fraction of CNTs, and effect of $1 / \mathrm{d}$ ratio of CNTs on the composite stiffness. In addition, upper and lower range limits of Young's modulus of CNTs, a working range of response can be obtained for composite material. Fig. A3 considered various $l / d$ ratios of the CNTs and $E_{f} / E_{m}\left(=E_{r}\right)$ ratios for various combinations to state that there was not much effect of $l / d$ ratio of CNT on the effective stiffness. This can be attributed to very small lengths of CNTs considered ( 0.5 to 15 microns) and randomly aligned CNTs display average constant-volume-behavior. Young's modulus of CNT is considered between $300 \mathrm{GPa}$ (lower curve in Fig. A3) to $1 \mathrm{TPa}$ (Upper curves in Fig. A3)

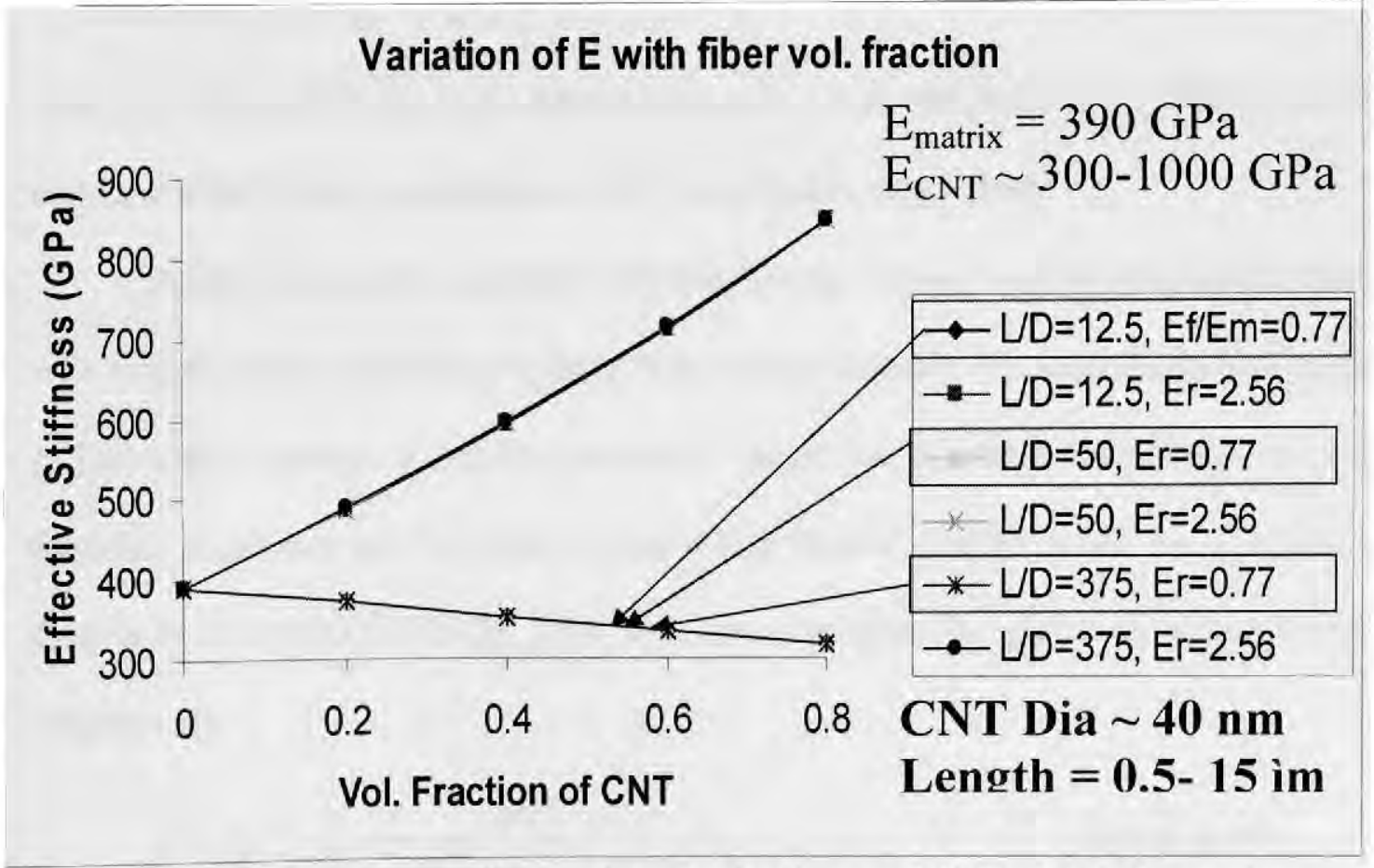

Fig. A3: Effective stiffness of randomly dispersed CNTs in $\mathrm{Al}_{2} \mathrm{O}_{3}$ matrix. 
Young's modulus of CNT ( $\left.\mathrm{E}_{\mathrm{CNT}}\right)$ is of critical importance since no significant effect of $1 / \mathrm{d}$ ratio plays a part in deciding $E_{11}$ of composite. But, at the same time, volume percent of CNT has stronger effect on tensile and shear stiffness of the composite.

Eshelby and Hashin Shtrikman approach do not consider L/D ratio or particulate geometry. As observed for randomly oriented fibers, that $1 / \mathrm{d}$ ratio do not impart much effect on determining the overall stiffness of the composite, this is an acceptable assumption. In addition linear elastic approach considered in this model is true in our case owing to brittle nature of the ceramic composites. Tremendous improvement in the properties of the CNT reinforced composite justifies the strengthening via CNT addition.

Rule of mixture (ROM), eq. A7, considers one-dimensional model to determine elastic modulus $\left(\mathrm{E}_{\mathrm{c}}\right)[44]$.

$$
E_{C}=V_{C N T} E_{C N T}+V_{m} E_{m} \quad \text { Equation A7 }
$$

where the $V_{C N T}$ and $V_{m}$ are the volume fraction of CNT, and matrix respectively, and $E_{C N T}$ and $E_{m}$ are the Young's modulus of CNTs and matrix respectively.

Eshelby approach considers effective Young's modulus of heterogeneous material with linearly elastic material response. It assumes randomly distributed spherical particles in the matrix leading to isotropic nature of properties. Standard bulk, shear and elastic modulus equations can be used in converting moduli dependencies, i.e, $\mathrm{K}=\mathrm{E} / 3(1-2 v)$; $\mathrm{G}=\mathrm{E} / 2(\mathrm{l}+\mathrm{v}) ; \mathrm{E}=9 \mathrm{KG} /(3 \mathrm{~K}+\mathrm{G})$. Bulk and shear modulus are given by eqs. $\mathrm{A} 8$ and $\mathrm{A} 9$ respectively:

$$
\bar{K}=K^{M}-\frac{f K^{M}}{\left[\frac{K^{M}}{K^{M}-K^{C N T}}-\frac{1+v^{M}}{3\left(1-v^{M}\right)}\right]}
$$




$$
\bar{G}=G^{M}-\frac{f G^{M}}{\left[\frac{G^{M}}{G^{M}-G^{C N T}}-\frac{2\left(4-5 v^{M}\right)}{15\left(1-v^{M}\right)}\right]}
$$

where $K^{M}, G^{M}$ are bulk and shear moduli of matrix, $v^{M}$ is matrix's Poisson's ratio, $K^{C N T}$, $G^{C N T}$ are bulk and shear moduli of CNTs, and $f$ is volume fraction of CNTs.

Hashin-Shtrikman shows narrowed bounds of isotropically heterogeneous material. Equations describing the bounds are expressed as:

$$
\begin{aligned}
& \frac{f}{1+\frac{(1-f)\left(K^{C N T}-K^{M}\right)}{\left(K^{M}+K^{-}\right)}} \leq \frac{\bar{K}-K^{M}}{K^{C N T}-K^{M}} \leq \frac{f}{1+\frac{(1-f)\left(K^{C N T}-K^{M}\right)}{\left(K^{M}+K^{+}\right)}} \\
& \frac{f}{\frac{f}{1+\frac{(1-f)\left(G^{C N T}-G^{M}\right)}{\left(G^{M}+G^{-}\right)}} \leq \frac{\bar{G}-G^{M}}{G^{C N T}-G^{M}} \leq \frac{f}{1+\frac{(1-f)\left(G^{C N T}-G^{M}\right)}{\left(G^{M}+G^{+}\right)}} \quad \text { Equation A10 }} \quad \text { Alion 1 }
\end{aligned}
$$

Condition I: If $\left(\mathrm{G}^{\mathrm{CNT}}-\mathrm{G}^{\mathrm{M}}\right)\left(\mathrm{K}^{\mathrm{CNT}}-\mathrm{K}^{\mathrm{M}}\right) \geq 0$

$$
\begin{aligned}
& \text { then } \mathrm{K}^{-}=4 \mathrm{G}^{\mathrm{M}} / 3 ; \mathrm{K}^{+}=4 \mathrm{G}^{\mathrm{CNT}} / 3 \\
& G^{-}=\frac{3}{2\left(\frac{1}{G^{M}}+\frac{10}{9 K^{M}+8 G^{M}}\right)} \\
& G^{+}=\frac{3}{2\left(\frac{1}{G^{C N T}}+\frac{10}{9 K^{C N T}+8 G^{C N T}}\right)}
\end{aligned}
$$

Condition II: If $\left(\mathrm{G}^{\mathrm{CNT}}-\mathrm{G}^{\mathrm{M}}\right)\left(\mathrm{K}^{\mathrm{CNT}}-\mathrm{K}^{\mathrm{M}}\right) \leq 0$

$$
\begin{aligned}
& \text { then, } \mathrm{K}^{-}=4 \mathrm{G}^{\mathrm{CNT}} / 3 ; \mathrm{K}^{+}=4 \mathrm{G}^{\mathrm{M}} / 3 \\
& G^{-}=\frac{3}{2\left(\frac{1}{G^{M}}+\frac{10}{9 K^{(N T}+8 G^{M}}\right)}
\end{aligned}
$$




$$
G^{+}=\frac{3}{2\left(\frac{1}{G^{C N T}}+\frac{10}{9 K^{M}+8 G^{C N T}}\right)}
$$

Independency of particle shape in Hashin Shtrikman's approach, provides enough leverage in calculating the bounds without worrying about the aspect ratio, and the isotropy of the second phase. Fig. A4 compares the theoretical Young's modulus calculations by various approaches. Experimental modulus values of $\mathrm{Al}_{2} \mathrm{O}_{3}$-CNT coatings are compared herewith.

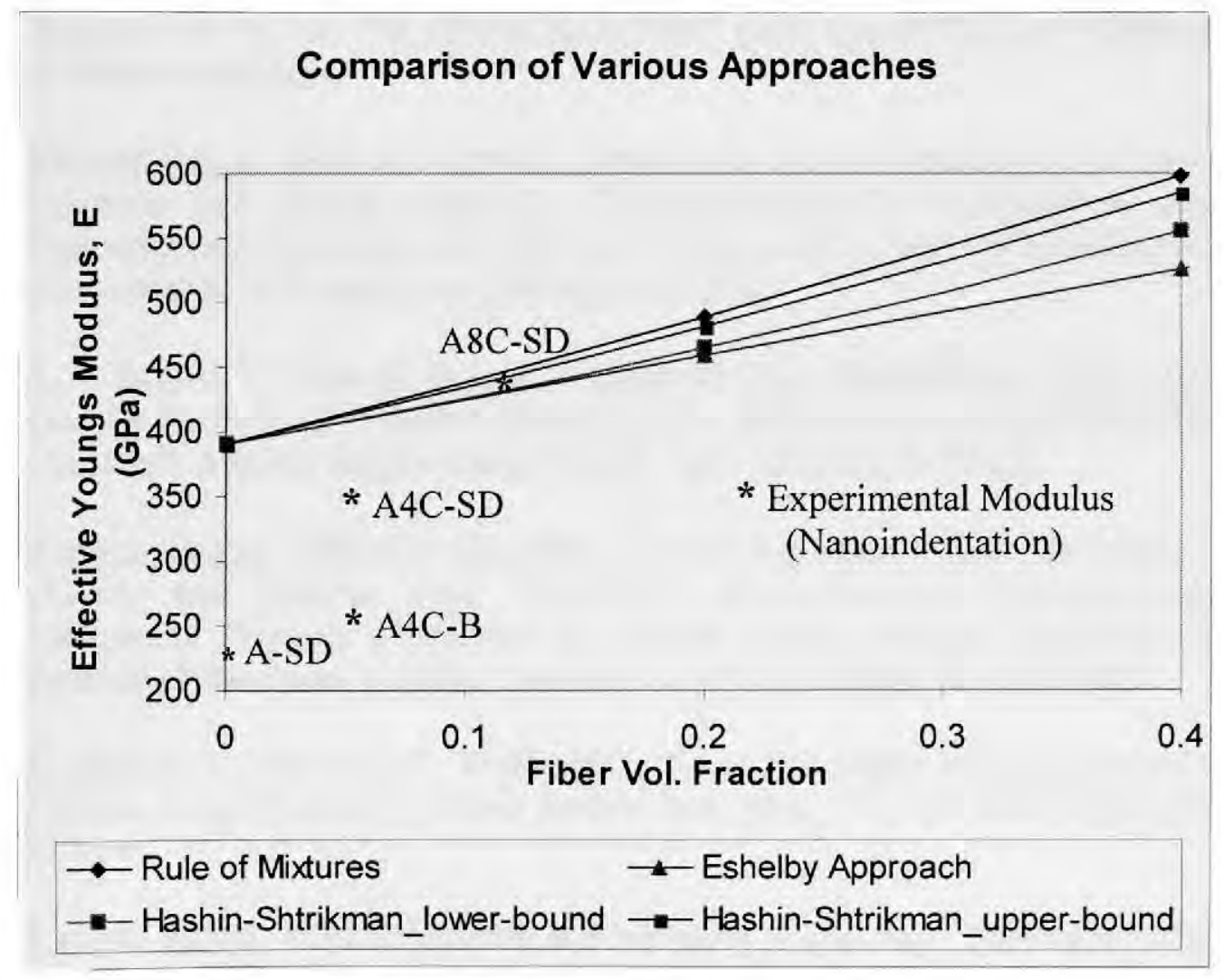

Fig. A4: Effective Young's modulus calculated by various micromechanics models.

Often plasma sprayed coatings depict reduced modulus owing to presence of lamellar microstructure and presence of defects (such as porosity, inclusions, etc) inherent to plasma spraying, as described in section 4.9.1. 
Appendix 3: This section includes list of directly related publications (peer-reviewed journal articles) during the current Ph.D. research work. First page of the published

papers directly related to the current research is provided here.

\section{Peer-Reviewed Journal and Proceedings}

1. Kantesh Balani, Srinivasa Rao Bakshi, Yao Chen, Tapas Laha, Arvind Agarwal, "Role of Powder Treatment and CNT Dispersion in the Fracture Toughening of Plasma-Sprayed Aluminum Oxide - Carbon Nanotube Ceramic Nanocomposite". Accepted in Journal of Nanoscience and Nanotechnology (Apr. 2007).

2. V. Viswanathan, T. Laha, K. Balani, A. Agarwal, S. Seal, "Challenges and Advances in Nanocomposite Processing Techniques", Materials Science And Engineering: R: Reports, vol. 54, No. 5-6, (2006), pp 121-285. (with Impact Factor of 10.52 next only to Nature Materials).

3. Kantesh Balani, Rebecca Anderson, Tapas Laha, Melanie Andara, Jorge Tercero, Eric Crumpler and Arvind Agarwal, "Plasma-Sprayed Carbon-Nanotube Reinforced Hydroxyapatite Coatings and Their Interaction with Human Osteoblasts In Vitro", Biomaterials, Vol. 28, No. 4, (2007) pp 618-624.

4. S. R. Bakshi, T. Laha, K. Balani, A. Agarwal and J. Karthikeyan, "Effect Of Carrier Gas On Mechanical Properties And Fracture Behaviour Of Cold Sprayed Aluminium Coatings". Surface Engineering, Vol. 23, No.1, (2007), pp. 18-22.

5. Kantesh Balani, Gabriela Gonzalez, Arvind Agarwal, Robert Hickman, J. Scott O'Dell, and Sudipta Seal, "Synthesis, Microstructural Characterization and Mechanical Property Evaluation of Vacuum Plasma Sprayed Tantalum Carbide", Journal of American Ceramic Society, Vol. 89 (4), (2006), pp 1419-1425.

6. K. Balani, A. Agarwal, T. McKechnie, "Near Net Shape Fabrication via Vacuum Plasma Spray Forming", Trans Indian Inst. Met., Vol. 59, No.2 April (2006), pp 237-244.

7. Kantesh Balani, Arvind Agarwal, and Narendra B. Dahotre, "Molecular Modeling of Metastable $\mathrm{FeB}_{49}$ Phase Evolution in Laser Surface Engineered Coating", Journal of Applied Physics, Vol. 99, (2006), DOI: 10.1063/1.2172704.

8. K. Balani, A.Agarwal, S. Seal, J. Karthikeyan, "Transmission Electron Microscopy of Cold Sprayed 1100 Aluminum Coating", Scripta Materialia, Vol. 53 (2005), pp 845850 . 
9. K. Balani, T. Laha, A. Agarwal, J. Karthikeyan, and N. Munroe, "Effect of Carrier Gases on Microstructural and Electrochemical Behavior of Cold-Sprayed 1100 Aluminum Coating". Surface and Coatings Technology, Vol. 195, 2-3, 31 (May 2005), pp 272-279.

10. T. Laha, K. Balani, A. Agarwal, S. Patil, S. Seal, "Synthesis of Nanostructured Aluminum Oxide Powders by Plasma Engineering". Metallurgical and Materials Transactions A, Vol. 36 A, 2, (Feb. 2005), 301-309.

11. Kantesh Balani, Yao Chen, Sandip P. Harimkar, Narendra B. Dahotre, Arvind Agarwal, "Tribological Behavior of Plasma Sprayed Carbon Nanotube Reinforced Hydroxyapatite-Coating in Physiological Solution". Accepted in Acta Biomaterialia (Jun. 2007). 


\section{Role of Powder Treatment and CNT Dispersion in the Fracture Toughening of Plasma-Sprayed Aluminum Oxide - Carbon Nanotube Ceramic Nanocomposite}

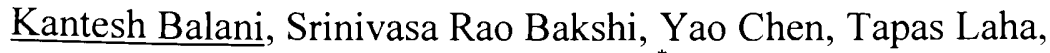
Arvind Agarwal*

Mechanical and Materials Engineering Department, EAS 3464, 10555 W. Flagler St., Miami, FL-33174, USA

\section{ABSTRACT:}

$\mathrm{Al}_{2} \mathrm{O}_{3}$ ceramic reinforced with 4-wt.\% multiwalled carbon nanotube (CNT) is plasma sprayed for improving the fracture toughness of the nanocomposite coating. Two different methodologies of CNT addition have been adopted in the powder feedstock to assist $\mathrm{CNT}$ dispersion in the nano- $\mathrm{Al}_{2} \mathrm{O}_{3}$ matrix. First, spray-dried nano- $\mathrm{Al}_{2} \mathrm{O}_{3}$ agglomerates are blended with $4 \mathrm{wt} . \% \mathrm{CNT}$ as powder-feedstock, which is subsequently plasma sprayed resulting in the fracture toughness improvement of $19.9 \%$. Secondly, spray dried composite nano- $\mathrm{Al}_{2} \mathrm{O}_{3}$ and $4 \mathrm{wt} . \% \mathrm{CNT}$ powder was used as feedstock for attaining improved dispersion of CNTs. Plasma sprayed coating of composite spray dried powder resulted in increase of $42.9 \%$ in the fracture toughness. Coating synthesized from the blended powder displayed impact alignment of CNTs along splat interface, and CNTs chain loop structure anchoring the fused $\mathrm{Al}_{2} \mathrm{O}_{3}$ melt whereas coating synthesized from composite spray dried powder evinced anchoring of CNTs in the solid state sintered region and CNT mesh formation. Enhanced fracture toughness is attributed to significance of CNT dispersion.

Accepted for publication (Apr. 2007) in the Journal of Nanoscience and Nanotechnology

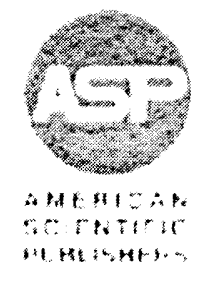

" Corresponding Author: Dr. Arvind Agarwal, Ph: 305-348-6533, Fax: 305-348-1932, Email: agarwala@fiu.edu 


\title{
Challenges and advances in nanocomposite processing techniques
}

\author{
V. Viswanathan ${ }^{a}$, T. Laha ${ }^{b}$, K. Balani ${ }^{b}$, A. Agarwal ${ }^{\text {b }}$, S. Seal ${ }^{a, *}$ \\ 'Advanced Materials Processing and Analysis Center, Department of Mechanical, Materials and Aerospace Engineering, \\ Nanoscience and Technology Center. University of Central Florida, Orlando, FL 32826, USA \\ "Mechanical and Matterials Engineering, Florida International University, Miami, FL 33174, USA
}

Available online 6 February 2007

\begin{abstract}
Of late, nanotechnology seems to be rapidly thrusting its applications in all aspects of life including engineering and medicine. Materials science and engineering has experienced a tremendous growth in the field of nanocomposite development with enhanced chemical, mechasical, and physical properties. A wide array of research has been conducted in the processing of nanocomposites. Consolidation of these systems from loose paricles to bulk free form entities has always been a challenge. To name a few, traditional consolidation techniques such as cold pressing and sintering at high temperatures, hot pressing, and hot isostatic pressing have strong limitations of not being able to retain the nanoscale grain size due to the excessive grain growth during processing. This article reviews in detail the results from numerous studies on various methods for manufacturing nanocomposites with improved properties and rctaincd nanostnuctures. Both challenges and recent advances are discussed in detail in this review. (C) 2006 Elsevier B.V. All rights reserved.
\end{abstract}

Keywords: Nanostructures; Processing tecluniques: Mechunical properties; Processing challenges

\section{Contents}

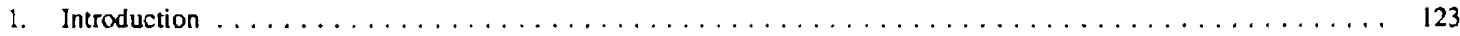

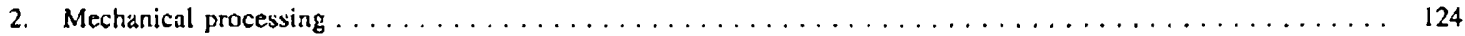

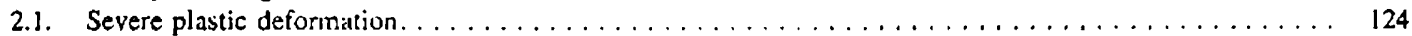

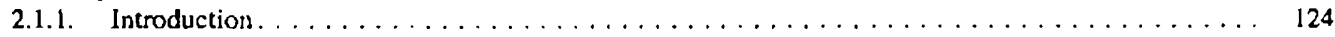

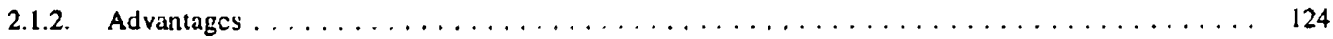

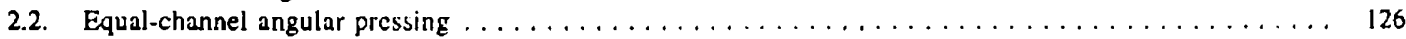

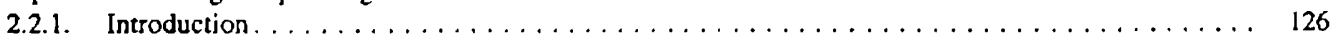

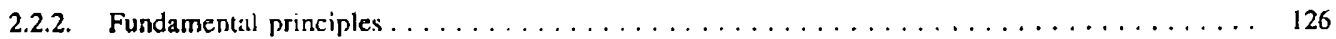

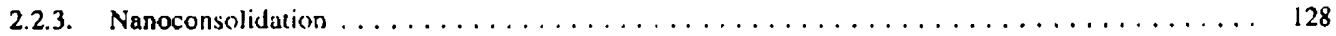

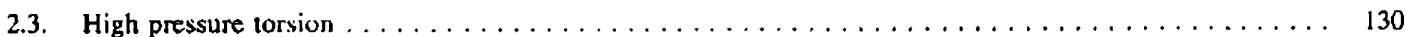

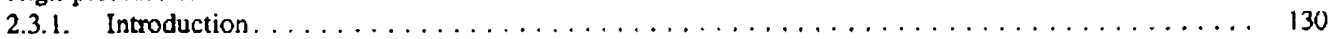

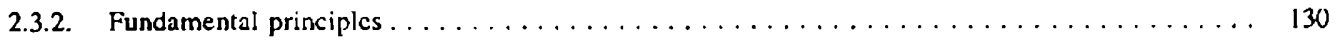

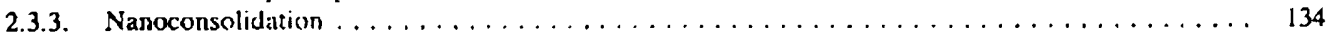

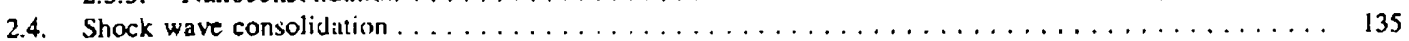

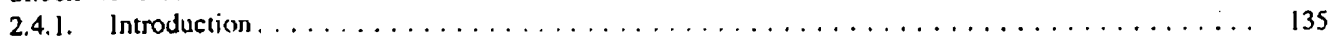

\footnotetext{
- Corresponding suthor. Tel.: +1 $4078821119 ;$ fax: +14078821462.

E.mail acdiress: ssea]@nibil.ucf.eclu (S. Seal).
}

0927-796X \$ - see front multer (1) 2006 Elsevier B.V. All rights reserved. doi: $10.1016 /$ j.mer.2006.11.002 


\title{
Plasma-sprayed carbon nanotube reinforced hydroxyapatite coatings and their interaction with human osteoblasts in vitro
}

\author{
Kantesh Balani", Rebecca Anderson ${ }^{\text {' }}$, Tapas Laha ${ }^{a}$, Melanie Andara ${ }^{a}$, \\ Jorge Tercero ${ }^{\mathrm{a}}$, Eric Crumpler ${ }^{\mathrm{b}}$, Arvind Agarwal ${ }^{\mathrm{a}, *}$ \\ "Department of Mechanical and Materials Engincering. Filurila Intcrnutional University, EC 3464, 1055S West Flagler Street, Miami, FL 33I74. USA \\ 'Department of Biomedical Enginecrimit. Flowida Internutional Unicersity', EC 2601, los55 West Flagler Street. Miami. FL 33i74, USA \\ Received In August 2006; accepted 8 September 2006 \\ Available onliue 27 September 2006
}

\begin{abstract}
Carbon nanotubes (CNT) possess excellent mechanical propertics to play the role as reinforcement for imparting strength and toughness to brittle hydroxyapatite (HA) bioceranic coating. Howcver, lack of processing technique to uniformly distribuse multiwalled CNTs in HA coating and limited studics and sparse knowleoge evincing toxicity of CNTs has kepe researchers in dispute for long. In the current work, we have addressed these issues by (i) successfully distributing mulliwalled CNT reinforcement in HA coating using plasma spraying to improve the fracture toughness (by $56 \%$ ) and enhance crystallinity (by $27 \%$ ), and (ii) culiuring human osteoblast $\mathrm{hFOB} 1.19$ cells onto CNT reinforced HA coating to clicit its biocompalibility with living eclls. Unrestricted growth of human osteoblast hFOB 1.19 cells has been observed near CNT regions claining assistance by CNT surfaces to promoce cell growth and proliferation.
\end{abstract} (c) 2006 Elsevier Lid. All rights reserved.

Keywords: Hydroxyapatite coating: Carbun nanolube (CNT); Plasma spraying; Titanium alloy; Crystallinity; Human osteoblast

\section{Introduction}

Hydroxyapatite (HA), $\mathrm{Ca}_{10}\left(\mathrm{PO}_{4}\right)_{6}(\mathrm{OH})_{2}$, is all attractive biomaterial owing to its close chenical resemblance $(\mathrm{Ca} /$ $P=1.67)$ with bone and teeth $[1-3]$. Osteoblasts proliferate onto HA owing to its bioactivity and biocompatibility $[3,4]$ and therefore HA coatings have long been applied to dental implants, bone repair scallolds, skeletal implants. and body/bioinsert material [5]. Microstructure, crystilllinity, and phase composition of HA coating is critical in deciding its cell response and inechanical performance. Plasma sprayed HA coating often result in the generation of secondary phases such as tricalcium phosphate (TCP), tetracalcium phosphate (TTCP), calcium oxide (CaO), and amorphous calcium phosphates (ACPs) [6]. Though HA is very stable in the body environment, presence of secondary phases causes dissolution leading to degradation of the implant in vivo. Hence, higher crystallinity content is

\footnotetext{
"Corresponding author. Tel:: + 1305348 1701: Fax: + 13053481932
}

E-mail address: agarwala(nifiu.edu (A. Agirwal). required for the increased implant life. In addition. researchers have used carbon nanotubes (CNT), Ti-alloys, yttria stabilized zirconia (YSZ), $\mathrm{Ni}_{3} \mathrm{Al}$, and alumina $\left(\mathrm{Al}_{2} \mathrm{O}_{3}\right)$ reinforcements to $\mathrm{HA}$ coating [7-9] for improving its fracture toughness and wear resistance $[1,5,10-13]$.

Chen et al. investigated mechanical properties of laser processed HA-multiwalled CNT coating showing strong improvement in the fracture toughness and marginal improvement in the elastic modulus $[1,14]$. However, laser synthesized coating result in the formation of undesired TiC phase [1]. The purpose of addition of CNT was to enhance the mechanical performance of the coating without deteriorating the biocompatible properties of HA [15 17]. On the contrary, CNTs have also been debated as toxic [18] under organic environment $[1,19]$. Though in a recent study, osteosarcoma cell growth has been observed on functionalized CNT [20], researchers have also depicted micro patterning of CNTs to result directed growth of osteoblasts [21], or surface modification with DNA- and HA-nunostructured films for enhanced detection and biosensitivity [22]. Haddon et al. has concluded that CNT 


\title{
Effect of carrier gas on mechanical properties and fracture behaviour of cold sprayed aluminium coatings
}

\author{
S. R. Bakshi', T. Laha ${ }^{1}$, K. Balani ${ }^{1}$, A. Agarwal ${ }^{* 1}$ and J. Karthikeyan²
}

Two different coating of 1100 aluminium were cold sprayed onto similar substrates, using He and $\mathrm{He}-2 \mathrm{ON}_{2}(\mathrm{vol} . \%)$ mixture as carrier gases. Three point bend testing was carried out. The elastic moduli of the coatings were found to be close to each other and the substrate. The He processed coating showed higher fracture strength which was attributed to the higher degree of strain hardening. The He-2ON 2 processed coating falled at lower stress owing to its strain relaxed structure. The mode 1 fracture of the coating substrate system was found to be higher for the helium processed coating. The toughness was correlated to the microstructure. The delaminated coating showed a higher degree of brittle failure of the interface for the He processed coating.

Key worde: Coid spraying. 1100 akminum, Fracture surtace, Fracture bughess, Three point bend test, Elastic moduhs, Britie fracure. Delemination, Notch, Por osily. Carrier gas, Splas, Dislocation pile up, Subgrain tomation

\section{Introduction}

Cold spraying is a relatively new coating technique where metallic powder particles are aceelerated to very high velocitics $\left(6001500 \mathrm{~m} \mathrm{~s}^{-1}\right.$ ) by a carrice gas (He, $\mathrm{N}_{2}$ ) flowing at very high pressure (up to $3.5 \mathrm{MPa}$ ) and impuct the substrate through a converging diverging de laval nozzle. The carrier gas is prehcalod to a temperature between 300 and $800 \% \mathrm{C}$, partly in order to compensate for the cooling produced by expansion of the gas and to reduoe the density which in turn increases the sonic velocity, but below the melting point of the particles. The loss of kinetic energy on impact causes plastic deformation of the particles. This process is also known as cold kinetic spraying. The low temperature solid statc coating process climinates problems owing to oxidation and defocts oving to solidification. The disadvantage is that a large amount of carrier gas is lost, unless rocyclod, and that only plastically deform. able materials can be deposited. Many materials have becn depostited till date by cold spraying including pure metals, ${ }^{2}$ alloys 7 and composite materials. ${ }^{49}$ The exact mochanism of the bonding is not fully understood. It is belicved that inpact of the particles results in rupture of oxide layers which provides clean surfaces for bonding. Bonding has boen attributed to adiabatic shoar instabilities at particle/particle and partick/substrate interfaces owing to impact and has been modelled using linite chement analysis ${ }^{10.11}$ The parameters affecting the process and spraying efliciency are particle sizc, density

'Research Studert. Department of Mechanical and Ma br lats Engine ering. Flor ida Intern ational University. Miami, FL, USA

${ }^{2}$ Orrector of Pesearch and Deveiopment. ASB industries. Barbenton, OH USA

-Corresponding author, emal agarwata ofu.edu of particles, temperature of gas and density of gas and spraying angle, and various models have been proposed for the efloct of various parameters. ${ }^{12} 14$

In the authors' catier work, ${ }^{6,16}$ cold spraying of 1100 Al on 1100 Al substrate was reported. Two differnt carrier gases, namely $\mathrm{He}$ and $\mathrm{He} 20 \mathrm{~N}_{2}$ (vol. $-\%$, were used to study the effoct of carrier gas on the properties of the costing. It was observed that the $100 \% \mathrm{He}$ processed coating had higher hardness than the He $20 \mathrm{~N}_{2}$ processed coating. TEM analyis revealed dislocation pile-ups and oxide layers in the He processed coating. The $\mathrm{He} 20 \mathrm{~N}_{2}$ processed costing showed subgrain formation and a relaxed structure. ${ }^{16}$ There was more strain hardening in the He processod costing as compared with $\mathrm{He} 20 \mathrm{~N}_{2}$ processed costings. Potentiodynamic polarisition experiments in $0.9 \mathrm{pH}$ $\mathrm{H}_{2} \mathrm{SO}_{4}$ revealed that the corrosion current density was higher for the He processed coating than for the He $20 \mathrm{~N}_{2}$ processed coating indicating supcrior corrosion resislance of the $\mathrm{He} 20 \mathrm{~N}_{2}$ processed couting."

The purpose of the present work is to study the effect of the carier gas on the fracture strength and interfacial properties of the coating/subsrate and to establish correlation with the microstructure. Three point bend testing of the samples was carricd out. The elastic modulus and fracture propertics were studied.

\section{Experimental}

The schematic of the throe point bend test is shown in Fig. 1. A notch was introduced at the midpoint of the spocimen using a diamond suw. The initial crack length was messured by optical microscopy of the crosssoction. Table 1 cubulates the dimensions of the samples used.

For the throe point bend test geometry the elastic modulus of the coating/substrate system is given by the

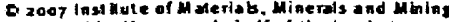

Publis hed by water on behalf of the inst in ule

recehod 24 Mor 2006: accepied is July 2006

$00110.8179 / 24329407 \times 161618$

Sufface Englneerlng 2007 vol 23 NOJ 1

Surtace Englneering sur408.3d 24/11:06 13:05:41

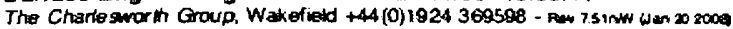




\title{
Synthesis, Microstructural Characterization, and Mechanical Property
} Evaluation of Vacuum Plasma Sprayed Tantalum Carbide

\author{
Kantesh Balani, Gabricla Cionzalez, and Arvind Agarwal ${ }^{\dagger}$ \\ Mechanical and Materials Engineering, Florida Internatonal University, Miami, Florida 33174 \\ Robert Hickman and J. Scot O'Dell \\ Plasma l'roceises Irx.. Huntivitle. Alabama 35811
}

Sudipta Seal

Advance Material Process and Analysis Center (AMPAC), Depanment of Mechanical, Materials and Aerospace Engineering (MMAE), Lniversity of Central Florida, Orlando, Florida 32816

\begin{abstract}
I anfalum carbide (IaC) is an ultra-high-lemperafure ceramic for potential a pplicatiom as protectice coating, furnace components. propulsion liners for space shutrles and aircrafis efc. Microstuctural and mechanical hehavior of vacuum plasmasprayed (VPS) $\mathrm{T}$ a C has ben invextigaled in the present studs. Apart from najor ' laC phase, microstructural definition elicidated $\mathrm{I}_{2} \mathrm{C}$, non stoichiometric $\mathrm{T}_{\mathrm{a}} \mathrm{C}_{\mathrm{x}}$ phases $(0.83 \leq x \leq 0.94)$. partial grain formation, polggonication of graims, and intomogeneous (fla ration in the sprayed structure. Near-isotropy in the fracture toughness ratio $\left(K_{\mathrm{ncin}} / K_{\mathrm{n} m \mathrm{x}}=1.01\right)$ is attributed to compact coal ling, fine-closed porosity, and distribution of nonstoichiometric phases.
\end{abstract}

\section{Introdiction}

$\mathrm{T}$ neracal protection systems, mozle throat inserts. and high erosion resistant thruaters require ultra-high-temperature capability of material for withranding exit gaser under propulsion environment.' 4 Candidate materials involve refractory metals, ceramic matrix composites, carbon carbon composites, cermets. and intermetallics. but no single material has the de. sired combination of physical, mechanical, and structural prop. erties. Ceramic oxides are chemically inert. but they are more brittle and suxceptible to thermal shock than non-axide ceram. ics. " On the one hand refractory metals excel in high-tempera. ture strength, wear, and etosion resistance, but on other hand possess Inv oxidution resistance, high density, poor machinability, and high cost 2 Ceramic borides posess high melting temperature, high hardness, and high thermal and electrical conductivity, but oxidation of boride leads to formation of $\mathrm{B}_{2} \mathrm{O}_{3}$ that has lower hoiling point $(18(x) \mathrm{C})$ leading to large voids in the oxide layer. In summary, increasing order of melting points is: alloys (up to $\sim 15\left(0^{\circ} \mathrm{C}\right.$ ). silicides (up to $\sim 24(x)^{\circ} \mathrm{C}$ ). oxides (up to $\sim 29 \times 1{ }^{\circ} \mathrm{C}$ ), tefractory metals, nitrides, and borides

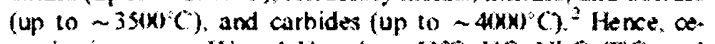
ramics in groups $I V$ and $V$ such as HFC, VC, NbC, TiC, and $\mathrm{TaC}$ are the contesting materials as they posses high melting point (close to $4(x)(C)$, high oxidation resistance. excellent ther. inal shock resistance. Iow coeficient of the rmal expansion. low vapor pressure at elevated temperatures. and good creep and fatigue properties. ${ }^{1,2}$ Therefore. TaC stands as a candidate ina.

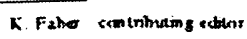

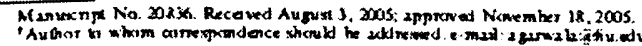

terial for next generation thermal heat protection. space air. crafts, automotive wear rexistant liners, and propulvion-exposed components, etc: 4

Powder metallurgy and conventional techniques such as hot isostatic pressing, direct metal oxidation, extrusion. and hot pressing have heen widely uned for $t$ be densification and near net shape (NNS) forming of ceramic materials. Though prom. ising, these tachniques lack the capability to fabricate contoured and thin wall structures owing to high melting temperature and brittleness of the ceramics. ${ }^{3}$ Hence, NNS processing of ultra. high-lemperature oramics (UHTC) as free-standing structure: becomes a problem when following conventional processing mute., Vacuum plasma spray formed tantalum carbide (I:C) provides an alternative to overcome fabrication hurdle of high melting point makerials and their briuleness. Ilasina plume reaches temperasures in excess of $10(\mathrm{~K} \times 0 \mathrm{~K}$ and impacts the pouders in molten or semi-molten states generating typical mechanically bonded lavered structure. ${ }^{4 a_{0} \text {. }}$ It is important to emphasize that much of the literature rexearch material for the applications of TaC, because of military applications, is sparingly available. 4.9 in the current work. microstructural chara. terization is cor related with the ohserved mechanical property of the vacuum plasma.sprayed (VI'S) TaC..

\section{Experinental Procedure}

As received TaC powder (with composition of $6.24 \mathrm{wt} \% \mathrm{C}$. $0.1034 \mathrm{w}(\% \mathrm{O}$, and rext $\mathrm{T} \mathrm{a})$, shown in Fig. 1 , has faceled mor. phology and is 1 z um in size. Spray drying of these tine pouder particles resulted in spherical agglomerates of porous cakes (Fig. 2) between 10 and $45 \mu \mathrm{m}$. which is adequate for plasma spraying. This spherical morphology and idealized size belp reduce inter particle friction. enta noe flowability of powder dur. ing plasma spray and the reby improve the density of the sprayed structure.

Figure 3 show's VPS-deposited frestanding TaC structure with a diame te of $50 \mathrm{~mm}$, wall thickness of $1.5 \mathrm{~mm}$, and height of $100 \mathrm{~mm}$. VTS deposition of freestanding $\mathrm{TaC}$ structure on graphice substrate was carried out at Ilasma Processes Inc. (Kuntwille, AL), using robot controlled Sulzer Metco Ell 140 $\mathrm{kW}$ plasma system (Wintertbur, Swiberland). A representative set of process parameters is presented in Table 1

Image. J'ro Plus, Version 5.1 imaging software (2XXM Media (ybernetica Inc. Silver Spring, MD) bas teen utilized for quin. titative image analysis of porosity distribution in the VPSTaC Sanning electron microsope (SEM)JEOL JSM SYWOLV. TO. kyo, Japan) with integrated energy-dispersive spectroscopy (EDS) bas been used for electron imaging and quantitative 


\title{
NEAR NET SHAPE FABRICATION VIA VACUUM PLASMA SPRAY FORMING
}

\author{
K.Balani", A.Agarwal" and T. McKechnie" \\ Mechanical and Materials Engineering. Florida International University, \\ 10555 W. Flagler Street, CEAS 3464, Miani, FL 33174, USA. \\ “Plasima Processes lnc., 4914 Moores Mill Road, Huntsville, AL 35811, USA \\ E-mail: kbalanigyahoo.com
}

(Received 3 November 2004 : in revised form 26 April 2006)

\begin{abstract}
Near net shape fahrication substantiates the manifestation of firal structure near to the desired stape, therefore can easily cut down the price of inished product contributed by machining. Thuugh conventional poudder metallurgy techniques are grominent in fabricating near bet shape structures with controlled porosity, it sill lags in producing thin walled complex shapes with funcrionally graded/ differential structures. Hence, fabrication of complex reear net shapex using Vacuum Plasma Spray (VPS) forming has emerged as innovative rapid protsyping technique for a variety of applications. In the presem work, the nuancess of near net shape tabrication hy VPS tochnique have theen explained. lew examples of spray formed stuctures such as hyperculectic aluminum-silicen, nanostructured aluminum oxide. and intermetallic iron aluminide thin stect thave been discussed. A detaited case study of VPS formed thin TaC: structure for ultrahigh temperature application has been elucidated via inar net shape tabrication.
\end{abstract}

\section{NEAR NET SHAPE FABRICATION USING VPS FORMING}

Owing to extreme britle nature, conventional fabrication techniques find difficult to slape refractory materials and intermetallics into desired shapcs. Moreover machining techniques incur extreme wastage of material and require dead time for the finishing of the strucural component ${ }^{1-2}$. Though few processes satisfy the near-net-slape labrication criteria, a serious limitation is posed by the extreme melting points of refractory materials. Ultrahigh temperature material application range between $2500-3300 \mathrm{~K}$ and therefore the selection of crucibles 10 hold the mett and help to form the finished product becomes quite difficult. Plasma spraying has been used as a versatile teclunique for depositing metals, alloys, polymers and ceramics as coatings ${ }^{3}$. Temperature in excess of $10,000 \mathrm{~K}$ are easily reached in the plasma flane, hence can melt any known material with greal ease. With an impact velocity of 1-3 Mach, plasma sprayed structure depict a typical mechanically bonded layered structure. VPS utilizes HF (high-frequency) starled,
DC plasma to heat and accelerale powder feedstock for deposition onto aegative shaped substrate/ mandrel for habricating positive shape structure, Fig. 1. Generally $\mathrm{Ar}$ is used as the primary plasma gas with secondary $\mathrm{He}$ or $\mathrm{H}_{2}$ gas for increased heal transfer. l'lasma gun can be manipulated by computer control in six axes of motion. With the cooling rates usually observed in the range of $10^{3}-10^{8} \mathrm{~K} / \mathrm{s}$, genteration of ultrafine grain microstructure and non-equilibrium phases is not so surprising in the Vacuum Plasma Spray (VPS) formed structured ${ }^{2.5}$. Both metals and ceratnics have been processed widh densities excetding $97 \%$ of theoretical densities 2.6 . Plasma gun and the mandrel arc compuler-controlled in VPS forming. lience fabrication of complex siructures can be designed accordingly. Deposition rates can go as ligh as $20 \mathrm{lb} / \mathrm{h}(9 \mathrm{~kg} / \mathrm{h})$. Near net sliapes, spray deposited onto preformed mandrel, help reduce the tinish machining of the deposited structure. Removal of mandrel fron the deposited structure hold a critical importance in the near-net fabrication of VPS formed structures. A comparison between VPS forming arks conventional $\mathrm{P} / \mathrm{M}$ processing route is schlematically 


\title{
Molecular modeling of metastable $\mathrm{FeB}_{49}$ phase evolution in laser surface engineered coating
}

\author{
K. Balani and A. Aganwaf) \\ Department of Mechanical and Materiols Engineering, Flortda Intermational University, EAS 3400, Miami. \\ Florida 31374 \\ Narendra B. Dahotre \\ Department of Materlalt Science and Engineering, The University of Tennersee, Knowville, \\ Tenne ssee 37996 and Materials Processing Group, Hetals and Ceranic Diviston, \\ Oak Ridge National Laboratory, Oak Ridge, Temessue 3783I
}

(Received 3 August 2005: accepted 17 January 2006; published online 17 February 2006)

\begin{abstract}
Interstitial ìn-boride phases have been a subject of resesrch interest for a long time owing to their useful propenties. Metastable $\mathrm{FeB}_{40}$ phase evolved during nonequilibrium laser surface engineering was investigated along with $\mathrm{FeB}, \mathrm{Fe}_{2} \mathrm{~B}$, and $\mathrm{Fe}_{3} \mathrm{~B}$ phases. Theoretical $x$-ray diffraction spectrum derived from uumerically constructed $\mathrm{FeB}_{49}$ crystol matched with the experimental diffraction pattern from loser surface engineered boride caxting on the steel substrate. Furthermore, employment of $a b$ initio sIESTA 1.3 molecular modeling for computation of total crystal energy elucidared instability of the $\mathrm{FeB}_{40}$ phase. The generation of thermodynamically nonequilibrium phase $\mathrm{FeB}_{49}$ olong with $\mathrm{Fe}_{3} \mathrm{~B}$ phase was further confirmed by selected area diffraction and tigh-resolution transmission electron microscopy analyses. 2006 American Institute of Physics. [DOI: $10.1063 / 1.2172704]$
\end{abstract}

\section{INTRODUCTION}

Lasers have been used for surface modification for improvement in material properties such as resistance to wear, friction, erosion, fatigue, corrosion, and high-temperature oxidation. ${ }^{1 \rightarrow}$ Laser material processing involves extremely high cooling rake $\left(10^{5}-10^{8} \mathrm{~K} / \mathrm{s}\right)$ that results in the generstion of metastable and nonequilibrium phases due to extended solid solubility. It is often observed that these phases are unknown to equilibrium phase diagram. ${ }^{1-6}$

In our earlier work, we have synthesized $\mathrm{TiB}_{2}$ coating on AISI 1010 steel substrate using a $2.5 \mathrm{~kW}$ continuous wave neodynium-doped ytrium aluminum garne (Nd:YAG) laser. ${ }^{1.24}$ Rapid cooling and solidification during laser processing generated "composite" coating consisting of borides in iron-rich matrix $\mathrm{TiB}_{2}$ was the major phase along with $\mathrm{TiB}$ and iron borides such as $\mathrm{FeB}, \mathrm{Fe}_{3} \mathrm{~B}$, and $\mathrm{FeB} \mathrm{B}_{40}$. 1.24

In continuation of the above mentioned effors, in the present work, phases of iron-boride system ( $\mathrm{FeB}, \mathrm{Fe}_{2} \mathrm{~B}$, $\mathrm{Fe}_{3} \mathrm{~B}$, and $\mathrm{FeB}_{49}$ phases) were considered for $a b$ inirio total energy simulation using smesta 13 (Spanish initiative for electronic simulations with thousands of atoms) molecular modeling package. Evolution of rarely reported metastable $\mathrm{FeB}_{49}$ phase was further validated employing high-resolution transmission electron microscopy (HRTEM). An effort was made to compare these phases with theoretically generaced Fo-B phases and correlate the total energy to the relative stability of the structure.

Apart from major $\mathrm{TiB}_{2}$ pealks, experimental x-ray diffraction (XRD) spectrum depicted some extra peaks corresponding to metastable phases. ${ }^{12.4}$ These extra peaks were

\footnotetext{
Author to whoto corrempondence stould be addressed: electromic rasil:
} agarwela ofiu.ed identified for $\mathrm{Fe}_{3} \mathrm{~B}, \mathrm{FeB}_{49}$ and $\mathrm{Fe}_{2.12} \mathrm{~B}_{103} 39$ phases. The experimentally obxained XRD partern was compared with theoretically computed XRD pattern (Fig. 1) for confirmation of the presence of $\mathrm{FeB}_{49}$ and $\mathrm{Fe}_{212} \mathrm{~B}_{103,31}$ phases. $\mathrm{FeB}_{49}$ crystal construction [Fig. 2(a)] and its computed XRD pattern were obrained using CANJNE 3.1 (Divergents A, Compiegne, France) crystallographic softw are. The strongest peak corresponding to $\mathrm{FeB}_{40}$ appeared to be overlapped by (001) peak of $\mathrm{TiB}_{2}$. Even though Toble I presented lattice parameters of various phases in laser synthesized composite conting, the primary interest of the present study resides with the characterization of metastable $\mathrm{FeB}_{49}$ phase, which so far is not reported in the open literature.

Thermodynamic calculations have shown that the free energy of $\mathrm{TiB}_{2}$ is the least and therefore is a most stable

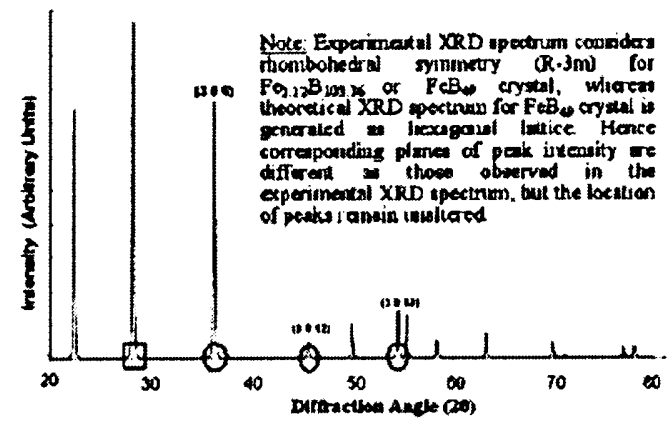

FIG. 1. (Color online) Theoretical x-rny diffuction spectrum of $\mathrm{FeB}_{\infty 0} \mathrm{cry}$ tal os seed is Pig. 2(n). All the aperimental peats for FeBas phase are matched with circles. The peak murbed with rectingle overlapped with toa jor $(\infty 1)$ peak of $\mathrm{TB}_{2}$ 


\title{
Transmission electron microscopy of cold sprayed 1100 aluminum coating
}

\author{
K. Balani ${ }^{\text {a }}$, A. Agarwal ${ }^{\text {a, }}$, S. Seal b, J. Karthikeyan ${ }^{\text {c }}$

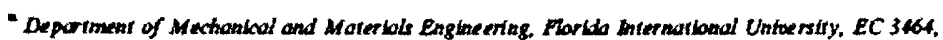

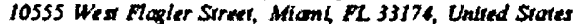

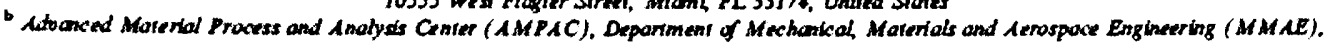

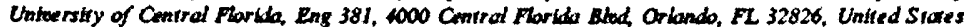 \\ "ASA Intesiries Iac., Burberron OH 4203, Unired Stores
}

Received 28 Pebruary 2005; received in reviead form 11 April 2005; acceptod 3 Juse 2005

Available online 6 July 2005

\begin{abstract}
Abrtract
Cold-spraying of 1100 Al powder particles onto 1100 Al substraxe was acomplished using carrier gases 100 vol.\% He and a mixf ure of He-20 vol.\% $\mathrm{N}_{2}$. TEM characterization correlates very well the cocurrence of dislocations, presence of oxide, and surface instability phenomenon with observed mechanical and corrosion properties of the cold-sprayed coating. - 2005 Acta Materialia Inc. Published by Elsevier Lid. All rights reverved.
\end{abstract}

Reyworts: Cold sprayige: 1100 Ahminum conting: TEM

\section{Introduction}

In the $1990 \mathrm{~s}$, the cold spraying process emerged in North America, having been developed in the mid1980 s by the Siberian Division of the Russia Academy at the Institute of Theoretical and Applied Mechanics [1-3]. A high-pressure $\left(\sim 3.5 \times 10^{6} \mathrm{~N} / \mathrm{m}^{2}\right)$ supersonic gas jet is utilized for accelerating fine powder particles to above a critical velocity $(\sim 500-1200 \mathrm{~m} / \mathrm{s})$, to deposit them as coatings [1-8]. The kinetic energy subsequently dispensed during the impact of the powder particle with the substrate ruptures the surface oxide, plastically deforms the particle and it approaches the clean surface of the substrate, thereby bonding particle as a deposition coating [9].

In our earlier work, 100 vol. $\%$ helium (He) and a mixture of helium and 20 vol. $\%$ nitrogen ( $\mathrm{He}-20$ vol. $\% \mathrm{~N}_{2}$ )

\footnotetext{
- Corresponding author. Tel.: +1 3053481301 ; fax: +1305348 1932.

Emoll addres: agrwahigin odv (A. A ganwal).
}

were used as carrier gases for depositing $1100 \mathrm{Al}$ onto 1100 Al substrate via the cold spray technique [3]. The higher sonic velocity and higher degree of tamping in 100 vol. \% He when compared to that of $\mathrm{He}-20 \mathrm{vol} . \%$ $\mathrm{N}_{2}$ carrier gas resulted in denser and harder coatings $[3,10]$. The addition of $\mathrm{N}_{2}$, being a diatomic gas, into He increased the enthalpy of the carrier gas for better heat-transfer with spray particles [11]. However, addition of $\mathrm{N}_{2}$ in He reduced the velocity of sprayed particles due to the heavier atomic mass resulting in coatings with reduced density and hardness [3]. Also, 100 vol.\% He processed coating displayed inferior corrosion resistance when compared to that of $\mathrm{He}-20 \mathrm{vol} \%$ $\mathrm{N}_{2}$ at $0.9 \mathrm{pH}$ using sulfuric acid $\left(\mathrm{H}_{2} \mathrm{SO}_{4}\right)$ as an electrolyte [3]. Both the cold-sprayed coatings were more corrosion resistant compared to the 1100 Al substrate.

In the present study, high magnification scanning electron microscopy (SEM) and transmission electron microscopy (TEM) were utilized to elucidate and substantiate results and findings of our earlier work [3]. TEM imaging educed dislocation piling, surface

$13396462 / 5$ - see front matter O 2005 Acta Materialin Inc. Publiabed by Elsevies Lid. Al rights reserved. dod: 10.1016 j scriptamat 2005.06 .008 


\title{
Effect of carrier gases on microstnuctural and electrochemical behavior of cold-sprayed 1100 aluminum coating
}

\author{
K. Balani", T. Laha*, ^. Agarwal ${ }^{* *}$, J. Karthikcyan ${ }^{\mathrm{b}}$, N. Munroc

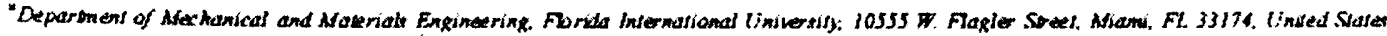 \\ "A.SB Intustrier, Inc. Barberion, Ohio 11203. Lnited Sures \\ Racisod 13 Fchnury 2004: acopted in revisod form 14 Junc 2004 \\ Arsilabic onlinc i Scprombet 2004
}

\begin{abstract}
Atrutract

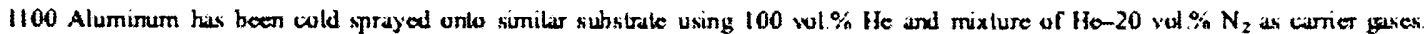

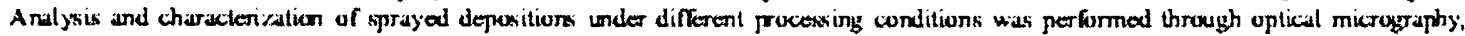

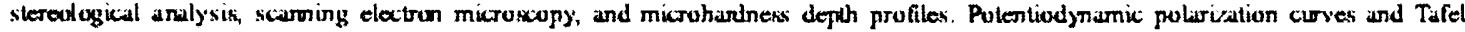
extrapolition experiments were carried at $0.9 \mathrm{pH}$ value using sulfuric acid $\left(\mathrm{H}_{2} \mathrm{SO}_{4}\right)$ as an elextrok jte. Helium processing condition displajed mure cumpact structure and higher handness for the coating when compared wo Ho-20 vol. \% $\mathrm{N}_{2}$ processing. Electroxhemical studies depicted

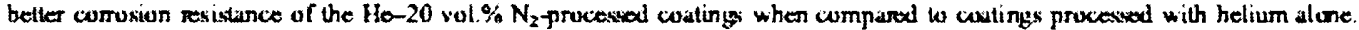
L 2004 Elverier B.V. All rights resenod

Kieynards. $X[C]$ Cold Sproy

\section{Introduction}

Cold spray is a relotively new coating process developed in the mid-1980x at the Institute of Theoretical and Applied Mechanics of the Siberian Division of the Russian Acaderny of Science in Nownsibirsk $[1,2]$. High-pressure cariet gas $(-3.5 \mathrm{MPo}$ or 500 psi) is required to impan high kivetic energy to the ponvder particles and achieve superanic velocities $\left(-5001200 \mathrm{~m} / \mathrm{s} ;\left[\begin{array}{ll}2 & 7\end{array}\right]\right)$. After the powder particles are $x$ celerated to these high velocities, they impact the substrote causing the thin surfoce oxide layers to rupture, plastically deform at surface, come in clase proximity of clean surfaces, thereby reonnsolidating the particles bonding them together. A high degree of plostic deformation occurs at the surfsce regions due in the impact of the accelerated particles onto the substrate. The source of bonding is the kinetic energy achieved by the particles under high-prexsure accelerating gas, to impinge onto substrate at temperarures tanging much below the melting point of the spray paricles.

\footnotetext{
- Corresponding author. Tel: +1 305 348 1701: Ex: +13053481932.

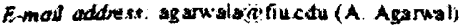

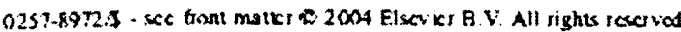
doi: 10. 1016ijsurfosst 2004.0x,028
No microstuctural evidence has been reported indicating the melting of the spray particles [6]. The bonding in particles in this process is attributed to the adiabatic shear instabilities the particle substrate or particle particle interfaces, caused due to the heavy particle impact [8].

The high velocity is developed by compressed gas flow (usulty helium, nitrogen, or the ir mixture) through high inlet pressure in a comverging diverging de Loval type of nozzle $[4,6]$. With increasing velocities, the particles undetgo from process of erosion and abrasion in depoxition onto substrate. Hence, critical velocity is a definite requirement for generoting sufficient kivetic energy to conse plastic deformation of the surfaces, thereby leading to depasition of spray material on substrate with a stong bonding $[6,7]$ Therefore, velocity of particles, attained priot to impact. holds a very important place in cold spraying. Brittle spray matetials, such as ceramics, do not undetgo the required plastic deformation in form a gond bond with the substrate, thereby the solution to the above problem is using ductile matrix or filler materials [9]. Hence, the oold-spray procexs is ideally suited for syrthesizing pure metallic cootings. This procexs resembles explosive welding in a manner that the 


\title{
Synthesis of Nanostructured Spherical Aluminum Oxide Powders by Plasma Engineering
}

\author{
T. LAHA, K. BALANI, A. AGARWAL, S. PATIL, and S. SEAL \\ lregularly shaped aluminum oxide particles were plasma atomized resulting in narrow size range dis- \\ tribution of spherical nanostructured powders. Cooling rates, on the order of $10^{6}$ to $10^{8} \mathrm{~K} / \mathrm{s}$, were \\ obtained from the different quenching medias, viz. air. waier, and liquid nitrogen. Plasma-engineered \\ powder particles developed nanosize crystallites, while solidification provided insight into the mor- \\ phological feasibility in refinement of grain size. X-ray diffraction (XRD) methods have been used \\ to quantify the crystallite size obtained with different quenching media. Raman peak shift validated \\ the $X$-ray analysis in anticipating the grain refinement with increasing cooling rates. Salient struc- \\ tural morphology characteristics and a detailed understanding of spheroidized plasma-sprayed alu- \\ mina powders were analyzed through scanning electron microscopy (SEM) studies. Formation of \\ nanograins, novel metastable phases, and amorphous structure were endorsed by transmission elec- \\ tron microscopy (TEM) investigations.
}

\section{INTRODUCTION}

NANOSTRUCTURED materials exhibit superior properties such as excellent strength, toughness, and hardness, as obvious from the presence of refined grains. ${ }^{11]}$ The nanostructured coatings/structures experience superior and novel properties; thus, the importance of achieving such tremendous improvement in performance cannot be neglected by researchers. Plasma spraying is one of the methods used to form nanocomposite structure, but it must overcome obstructing and difficult processing challenges. ${ }^{|2-6|}$ The first challenge is that the low mass and poor flow characteristics restrict the smooth flow of individual nanoparticles in the spray nozzle during thermal spraying. ${ }^{[2]}$ In addition, nanosized powders tend to agglomerate and form clogs in the plasma gun nozzle, hindering the smoothness of the flow and thereby imparting nonuniform coating. The other challenge is to retain the nanograins in the powder because grains coarsen, experiencing the high temperatures in the plasma flame. Several techniques are being developed to get nanocoating/composites by plasma such as enploynent of spray dried agglomerates of nanopowders, blending and spraying of bimodal powders with controlled plasma parameters, explosion of loose nanoagglomerates in plasma flame, etc. ${ }^{(2-6)}$ In this study, we are trying to modify the feedstock powder by two ways simultaneously, viz. spheroidization and nanocrystallization/grain refinement of spray particles. It is anticipated that synthesis of nanostructured ceramic spheres by plasma spray technique would result in development of an ideal free-flowing powder feedstock to synthesize nanostructured coatings, and small complex parts with improved physical properties. Spheroidization by

T. LAHA and K. BALANI, Graduate Research Assistants, and A. AGARWAL. Assistant Professor. are with the Department of Mechanical \& Malerials Enginoering, Fiorida Intemational University, Miami, FL 33174. Contact e-mail: agarwsla fiu edu S. PATIL, Graduate Research Assistant. and S. SEAL. Associate Professor, are with the Department of Mechanical. Materials and Aerospace Engineering. University of Cenural Forida, Orlando, FL. 32186.

Manuscript submitted March 29, 2004. plasma spraying has been studied earlier, ${ }^{[7,8.9]}$ but the novelty of this article lies in the idea of using different quenching media (viz. air, water, and liquid nitrogen) to achieve nanostructured grains in individual spherical powder particles through the plasma spraying process. $\mathrm{Al}_{2} \mathrm{O}_{3}$ (alumina) powder has been selected for the present study because spherical nanostructured alumina powder is an excelleni starting material for near-net-shape forming, spray powders for coatings and soft abrasives, surface adsorbents in chemical industries, biocompatible coatings, and as carriers for catalyst. ${ }^{(10)}$ This powder is also being used potentially in thermal insulation as well as in inertial confinement fusion of nuclear materials. ${ }^{[11]}$

Thermal plasma spraying has been used extensively within inany industries for a variety of applications because of its versatility to synthesize fune metal, alloy, ceramic, polymer, and composite powders. Plasma spheroidizing is a modified form of atomization technique, where the temperature reaches more than $15,000 \mathrm{~K}$ in plasma flame with gas velocities reaching speeds of 1 to 3 Mach. ${ }^{[12(3]}$ Because of the short residence time of the particle in the plasma, impurity limits in the product are generally low. ${ }^{[7]}$ The added beauty of the process is the use of inert carrier gases minimizing the chemical reaction of the processed powder, which possesses higher surface energy than the starting powder, due to its refined size. Processing of ceramics such as alumina powders becomes much easier since the temperatures achieved in plusma are much higher than the melting point of any known material. The surface tension on the molten particles, upon disintegration, fragments it into spheroids of fine particles added with quenching from water, and liquid nitrogen helps to nucleate and retain the nanocrystalline/grain structure of the particle. Plasma-spheroidized powder experiences rapid cooling, resulting in a high nucleation rate for solidified particles, without any significant time for grain growth.

\section{EXPERIMENTAL PROCEDURE}

Commercially available aluminum oxide powder $(99.8 \mathrm{pct}$ purity) of irregular shape in the 15 - to $45-\mu \mathrm{m}$ particle size 


\title{
Tribological Behavior of Plasma Sprayed Carbon Nanotube Reinforced Hydroxyapatite-Coating in Physiological Solution
}

Kantesh Balani ${ }^{1}$, Yao Chen ${ }^{1}$, Sandip P. Harimkar ${ }^{2}$, Narendra B. Dahotre ${ }^{2}$, Arvind Agarwal $^{1^{*}}$

'Department of Mechanical and Materials Engineering, Florida International University, Miami, FL 33174, USA

${ }^{2}$ Department of Materials Science and Engineering, University of Tennessee, Knoxville, TN 37996, USA

\begin{abstract}
Wear behavior of plasma sprayed carbon nanotube (CNT) reinforced hydroxyapatite (HA) coating is evaluated in the simulated body fluid environment. Apart from enhancing the fracture toughness and providing biocompatibility, CNT reinforced HA coating demonstrated superior wear resistance ( $>1.5$ times) compared to that of hydroxyapatite coating without $\mathrm{CNT}$. Initiation and propagation of microcracks during abrasive wear of plasma sprayed hydroxyapatite coatings was suppressed by CNT reinforcement. Surface characterization and wear studies have shown that in addition to acting as underprop-lubricant, CNTs provide reinforcement via stretching and splatbridging for enhanced abrasion resistance in vitro.
\end{abstract}

Accepted (Jun. 2007)

Acta Biomaterialia

\footnotetext{
* Corresponding author. Tel:305-348-1701, Fax:305-348-1932

Email address: agarwala@fiu.edu (Dr. Arvind Agarwal)
} 


\section{KANTESH BALANI}

1999

B. E., Metallurgical Engineering,

PSG College of Technology

Coimbatore, India

Best Outgoing Student

2001 M.Tech., Metallurgical and Materials Engineering, Indian Institute of Technology (IIT) Madras

Chennai, India

Sudharshan Bhat and S. Ananthramakrishnan Memorial Prize

Deutscher Akademischer Austausch Dienst (DAAD) Scholarship

2002 M.S., Materials Science and Engineering

University of Kentucky

Lexington, $\mathrm{KY}$

RCTF (Research Challenge Trust Fund) Fellowship

2003- Doctoral Candidate, Materials Science and Engineering

Florida International University

Miami, FL

Best PhD Student

Dissertation Year Fellowship

2006-07 Dean's Award of Highly Productive Doctoral Students

\section{Publications in Peer-Reviewed Journals:}

1. Kantesh Balani, Srinivasa Rao Bakshi, Yao Chen, Tapas Laha, Arvind Agarwal, "Role of Powder Treatment and CNT Dispersion in the Fracture Toughening of Plasma-Sprayed Aluminum Oxide - Carbon Nanotube Ceramic Nanocomposite". Accepted in Journal of Nanoscience and Nanotechnology (Apr. 2007).

2. V. Viswanathan, T. Laha, K. Balani, A. Agarwal, S. Seal, "Challenges and Advances in Nanocomposite Processing Techniques", Materials Science And Engineering: R: Reports, vol. 54, No. 5-6, (2006), pp 121-285. (with Impact Factor of 10.52 next only to Nature Materials).

3. Kantesh Balani, Rebecca Anderson, Tapas Laha, Melanie Andara, Jorge Tercero, Eric Crumpler and Arvind Agarwal, "Plasma-Sprayed Carbon-Nanotube Reinforced Hydroxyapatite Coatings and Their Interaction with Human Osteoblasts In Vitro", Biomaterials, Vol. 28, No. 4, (2007) pp 618-624.

4. Srinivasa R. Bakshi, Kantesh Balani, Tapas Laha, Jorge Tercero, Arvind Agarwal, "Nano-mechanical and Nano-scratch Characterization of UHMWPE and UHMWPE- 
5 wt.\% MWNT coatings on a steel substrate." (Accepted in the Journal of Minerals, Metals, and Materials (JOM) for July 2007 Issue).

5. S. R. Bakshi, T. Laha, K. Balani, A. Agarwal and J. Karthikeyan, "Effect Of Carrier Gas On Mechanical Properties And Fracture Behaviour Of Cold Sprayed Aluminium Coatings". Surface Engineering, Vol. 23, No.1, (2007), pp. 18-22.

6. Kantesh Balani, Gabriela Gonzalez, Arvind Agarwal, Robert Hickman, J. Scott O'Dell, and Sudipta Seal, "Synthesis, Microstructural Characterization and Mechanical Property Evaluation of Vacuum Plasma Sprayed Tantalum Carbide", Journal of American Ceramic Society, Vol. 89 (4), (2006), pp 1419-1425.

7. K. Balani, A. Agarwal, T. McKechnie, "Near Net Shape Fabrication via Vacuum Plasma Spray Forming”, Trans Indian Inst. Met., Vol. 59, No.2 April (2006), pp 237-244.

8. Kantesh Balani, Arvind Agarwal, and Narendra B. Dahotre, "Molecular Modeling of Metastable $\mathrm{FeB}_{49}$ Phase Evolution in Laser Surface Engineered Coating", Journal of Applied Physics, Vol. 99, (2006), DOI: 10.1063/1.2172704.

9. K. Balani, A.Agarwal, S. Seal, J. Karthikeyan, "Transmission Electron Microscopy of Cold Sprayed 1100 Aluminum Coating”, Scripta Materialia, Vol. 53 (2005), pp 845850 .

10. K. Balani, T. Laha, A. Agarwal, J. Karthikeyan, and N. Munroe, "Effect of Carrier Gases on Microstructural and Electrochemical Behavior of Cold-Sprayed 1100 Aluminum Coating". Surface and Coatings Technology, Vol. 195, 2-3, 31 (May 2005), pp 272-279.

11. T. Laha, K. Balani, A. Agarwal, S. Patil, S. Seal, "Synthesis of Nanostructured Aluminum Oxide Powders by Plasma Engineering". Metallurgical and Materials Transactions A, Vol. 36 A, 2, (Feb. 2005), 301-309.

12. Kantesh Balani, and Fuquian Yang, "Creep behavior of $90 \mathrm{~Pb}-10 \mathrm{Sn}$ alloy". Physica Status Solidi, 198, 2003, 387-394.

13. P. Gopalakrishnan, S. S. Ramakrishnan, Kantesh Balani, Amit Arora and Pranav Joshi, "Kinetic Study of Boriding Processes", Technology, Dec. 2000, p 20-25.

14. Kantesh Balani, Yao Chen, Sandip P. Harimkar, Narendra B. Dahotre, Arvind Agarwal, "Tribological Behavior of Plasma Sprayed Carbon Nanotube Reinforced Hydroxyapatite-Coating in Physiological Solution". Accepted in Acta Biomaterialia (Jun. 2007). 